VON KARMAN INSTITUTE FOR FLUID DYNAMICS

Aeronautics and Aerospace Department

Universitat Politècnica de CATALUnya

Escola Superior d’Enginyeries Industrial, AERonÀutica I Audiovisual de Terrassa

\title{
Assessment of Gas-Surface Interaction Modelling for Lifting Body Re-Entry Flight Design
}

September 2017

Thesis presented by Dipl.-Ing. Alan Viladegut Farran in order to obtain the degree of Doctor en Mecànica, Fluids $i$ Aeronàutica, Universitat Politècnica de Catalunya, Barcelona, 26 September 2017.

Promoters:

Prof. Olivier Chazot (von Karman Institute for Fluid Dynamics, Belgium) and Prof. Roberto M. Flores (Universitat Politècnica de Catalunya, Spain)

Doctoral Committee:

Dr. Jean-Luc Vérant (Office National d'Etudes et de Recherches Aérospatiales, France), Chairman of the commission. Prof. Miguel Mudarra (Universitat Politècnica de Catalunya, Spain), Secretary of the commission. Prof. Paolo Barbante (Politecnico di Milano, Italy), Comissioner.

Prof. Thierry Magin (von Karman Institute for Fluid Dynamics, Belgium), Reserve commisioner. Prof. Enrique Ortega (Universitat Politècnica de Catalunya, Spain), Reserve commisioner. 



\section{Acta de calificación de tesis doctoral}

\section{Curso académico:} Nombre y apellidos

Programa de doctorado

Unidad estructural responsable del programa

\section{Resolución del Tribunal}

Reunido el Tribunal designado a tal efecto, el doctorando / la doctoranda expone el tema de la su tesis doctoral titulada

Acabada la lectura y después de dar respuesta a las cuestiones formuladas por los miembros titulares del tribunal, éste otorga la calificación:
$\square$ NO APTO
APROBADO
$\square$ NOTABLE
SOBRESALIENTE

\begin{tabular}{|l|l|l|l|}
\hline \multicolumn{2}{|l|}{ (Nombre, apellidos y firma) } & \multicolumn{2}{l|}{ (Nombre, apellidos y firma) } \\
Presidente/a & (Nombre, apellidos y firma) & Secretario/a & (Nombre, apellidos y firma) \\
\hline (Nombre, apellidos y firma) & & Vocal \\
\hline
\end{tabular}

de

de

El resultado del escrutinio de los votos emitidos por los miembros titulares del tribunal, efectuado por la Escuela de Doctorado, a instancia de la Comisión de Doctorado de la UPC, otorga la MENCIÓN CUM LAUDE:

sí

$\mathrm{NO}$

\begin{tabular}{|l|l|}
\hline (Nombre, apellidos y firma) & (Nombre, apellidos y firma) \\
$\begin{array}{l}\text { Presidente de la Comisión Permanente de la Escuela de } \\
\text { Doctorado }\end{array}$ & $\begin{array}{l}\text { Secretario de la Comisión Permanente de la Escuela de } \\
\text { Doctorado }\end{array}$ \\
\hline
\end{tabular}

Barcelona a de de 

Al meu pare Cosme i a la meva dona Aida, els peus a terra, el cor volant. 



\section{Acknowledgements}

My wish is that the reader enjoys this dissertation and that it brings him/her a better understanding on Gas-Surface Interaction (GSI) phenomena. Nonetheless, this journey through the VKI doctoral program has been long, and I wouldn't be able to address this topic without acknowledging people that were by my side during this research, because without them this thesis would not have been possible:

A big thank to Olivier Chazot for giving me the opportunity to develop my thesis in the Plasmatron facility. His office was always open and ready for discussion. A special mention to his telephone, most of the time ringing during each one of our conversations. Also to Roberto Flores, director of this thesis in UPC, who allowed me to keep in contact with my university.

Following the experimental nature of this dissertation, I would like to mention also the contribution made by Pascal Collin. He did an excelent job running the Plasmatron and installing the probes. I will always remember his desperation when water leaks appear when making the vacuum in the chamber. I thank also the rest of the Plasmatron team: Bernd Helber and Damien Le Quang as radiation experts, and Işil Şakraker as advisor for calorimetric measurements.

Many CFD simulations appear in this dissertaion. For that, the advice of Vincent Van Der Haegen, my office mate, has been extremely useful to achieve a converged solution. Also, the flight extrapolation technique developed here deserves my gratitude to Fabio Pinna, whose expertise in CFD helped me to improve my IXV simulations and achieve the "Pinna Certification". I really enjoyed working with them.

Other VKI colleagues I want to mention are Alessandro Turchi, who helped me understand many aspects of GSI phenomena, Francesco Panerai who set the basis to develop this dissertation, and Georgios Bellas-Chatzigeordis to whom I encourage to continue with the catalytic model development and implementation in VKI numerical tools.

Last but not least, special thanks to my family. They encouraged me to pursue a doctorate degree and supported me in the most difficult moments. 



\section{Abstract}

Space re-entry is a challenging endeavor due to the harsh thermo-chemical environment around the vehicle. Heat flux being the reference parameter for Thermal Protection System design, the total energy transfer can significantly increase due to exothermic atomic recombination enhanced by TPS catalytic properties. The catalytic recombination coefficient modelling is critical for heat flux computation during TPS design.

This work assesses the VKI-Plasmatron capabilities to determine the recombination coefficient for catalytic model validation: from a deterministic reference catalytic model development for enthalpy characterization of the facility, to the identification of influencing parameters found in nonequilibrium boundary layers. Plasmatron test results encourage a flight extrapolation strategy development in order to link catalysis measured on ground, with catalysis appearing in flight. Such strategy being focused on off-stagnation point, it contributes to the future post-flight activities of the CATalytic Experiment on board of the Intermediate eXperimental Vehicle. Relevant data provided by IXV and CATE are also presented. 



\section{Contents}

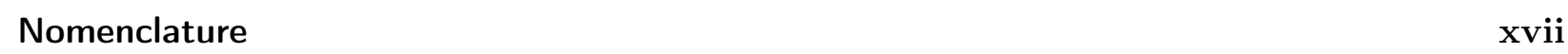

\begin{tabular}{lll}
\hline & Introduction & $\mathbf{1}$
\end{tabular}

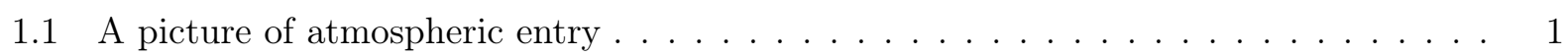

1.2 Physical models in hypersonics $\ldots \ldots \ldots \ldots \ldots \ldots \ldots \ldots$

1.3 Ground characterization of GSI phenomena $\ldots \ldots \ldots \ldots \ldots$

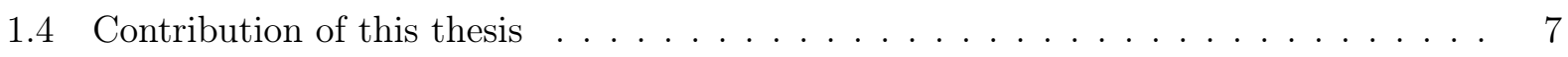

\begin{tabular}{|lll}
2 & State of the Art on Catalysis & 9
\end{tabular}

$2.1 \quad$ On catalytic modelling $\ldots \ldots \ldots \ldots \ldots \ldots \ldots$

2.1 .1 The macroscopic description . . . . . . . . . . . . . . . . . . . 10

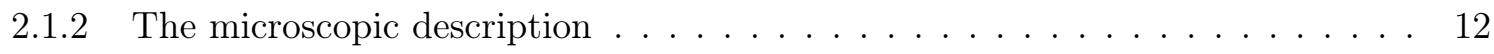

$2.2 \quad$ On facilities for surface catalysis determination $\ldots \ldots \ldots \ldots \ldots \ldots$

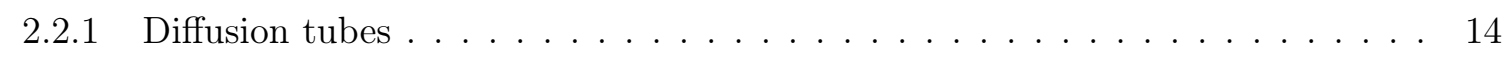

$2.2 .2 \quad$ Plasma wind tunnels $\ldots \ldots \ldots \ldots \ldots \ldots \ldots \ldots$

2.3 On flight extrapolation for catalysis $\ldots \ldots \ldots \ldots \ldots \ldots \ldots \ldots$

\begin{tabular}{|lll}
\hline & Enthalpy Characterization of the VKI-Plasmatron & 25
\end{tabular}

3.1 The enthalpy rebuilding method $\ldots \ldots \ldots \ldots \ldots \ldots \ldots \ldots$

3.2 The Mini-Max methodology . . . . . . . . . . . . . . . . . . . . . . . 34

3.3 The Mini-Max testing application $\ldots \ldots \ldots \ldots \ldots \ldots$

3.4 Reference recombination coefficient determination . . . . . . . . . . . . . . . . . . . . . 39

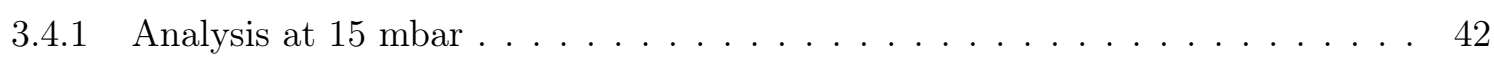

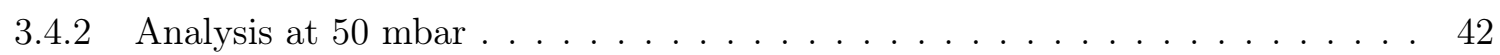

3.4 .3 Analysis at $100 \mathrm{mbar}$. . . . . . . . . . . . . . . . . . . 43

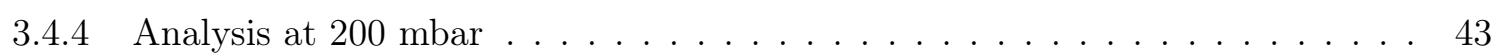

3.5 The Plasmatron reference catalytic model $\ldots \ldots \ldots \ldots \ldots \ldots \ldots$

\begin{tabular}{|lll}
\hline 4 & Catalysis in Different Flow Configurations & 47 \\
\hline
\end{tabular}

4.1 On the catalysis determination in non-equilibrium flows . . . . . . . . . . . . . 50

$4.1 .1 \quad$ Parametric analysis with NEBOULA $\ldots \ldots \ldots \ldots \ldots \ldots$

4.2 Testing configurations at the VKI-Plasmatron . . . . . . . . . . . . . . . 54

4.2 .1 Stagnation point: the Damköhler probes $\ldots \ldots \ldots \ldots \ldots \ldots$. . . . . . 55

4.2 .2 Off-stagnation point: the flat plate . . . . . . . . . . . . . 56

4.3 Catalysis determination at stagnation point $\ldots \ldots \ldots \ldots \ldots \ldots$

$4.3 .1 \quad$ Analysis of the Damköhler probes at $15 \mathrm{mbar} \ldots \ldots \ldots$. . . . . . . 62 
$4.3 .2 \quad$ Analysis of the Damköhler probes at $50 \mathrm{mbar} \ldots \ldots \ldots$. . . . . . . 63

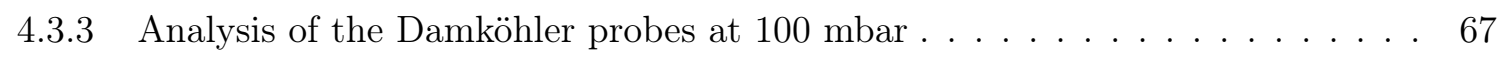

4.4 Catalysis determination at off-stagnation point $\ldots \ldots \ldots \ldots \ldots \ldots$

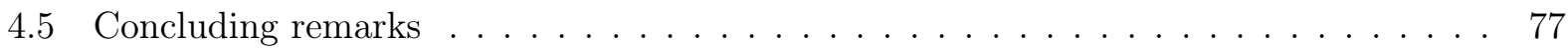

\begin{tabular}{|lll}
5 & Flight extrapolation at off-stagnation point & $\mathbf{7 9}$
\end{tabular}

5.1 The off-stagnation point extrapolation methodology $\ldots \ldots \ldots \ldots \ldots$. . . . . . 79

5.2 Application of the methodology $\ldots \ldots \ldots \ldots \ldots \ldots \ldots$

5.2 .1 Curvature effect . . . . . . . . . . . . . . . . . . . . . . . . . 84

5.2 .2 Scale effect . . . . . . . . . . . . . . . . . . . . . 88

5.3 Comparison of the boundary layer profiles $\ldots \ldots \ldots \ldots$. . . . . . . . . . 89

5.4 Validation of the methodology $\ldots \ldots \ldots \ldots \ldots \ldots \ldots \ldots$

5.4 .1 CFD assessment for flow characterization . . . . . . . . . . . . . . . . . 93

5.4 .2 Test campaign and results $\ldots \ldots \ldots \ldots \ldots \ldots \ldots \ldots$

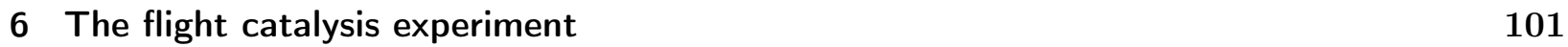

6.1 CATE design . . . . . . . . . . . . . . . . . . . . . . . 103

$6.2 \quad$ CATE implementation . . . . . . . . . . . . . . . . . . . . 104

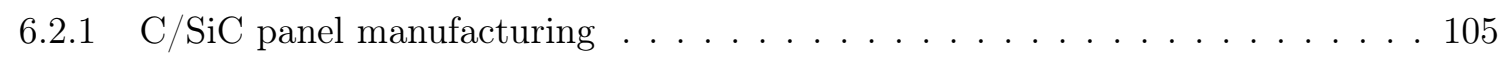

6.2 .2 Coating deposition by Vacuum Plasma Spraying. . . . . . . . . . . . . 106

6.2 .3 Thermocouple integration and calibration . . . . . . . . . . . . . . . . . . . . . . . . . . . . . . . . .

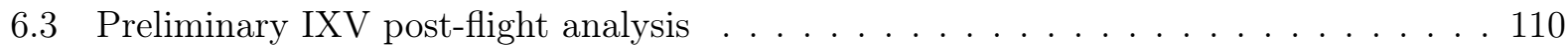

6.3 .1 Stagnation point . . . . . . . . . . . . . . . . . 110

6.3 .2 Off-stagnation point: CATE $\ldots \ldots \ldots \ldots \ldots \ldots \ldots \ldots \ldots$

6.3 .3 Thermal analysis for CATE post-processing . . . . . . . . . . . . . 118

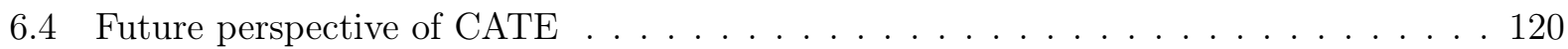

$\begin{array}{lll}7 \text { Conclusions } & 121\end{array}$

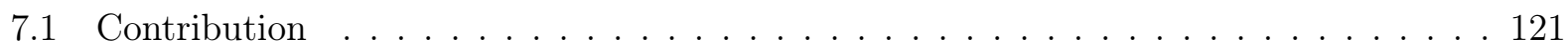

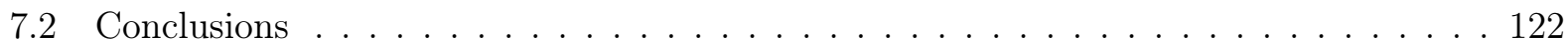

7.2 .1 The Mini-Max . . . . . . . . . . . . . . . . . . . . . . . 123

7.2 .2 The Damköhler probes and the flat plate . . . . . . . . . . . . . . . . . . 124

7.2 .3 The off-stagnation point extrapolation $\ldots \ldots \ldots \ldots$. . . . . . . . . 125

7.2 .4 The catalytic experiment CATE . . . . . . . . . . . . . . . . 125

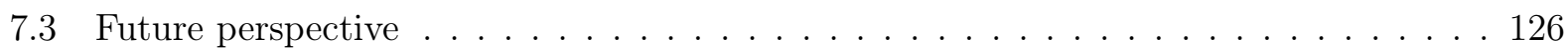

\begin{tabular}{|lr}
\hline A Dynamic pressure measurements & 129
\end{tabular}

\begin{tabular}{|ll}
\hline B NDP database for stagnation point test campaigns & 131
\end{tabular}

\begin{tabular}{|ll|}
\hline C List of Damköhler probes' non-dimensional parameters & 137
\end{tabular}

\begin{tabular}{lr}
\hline Bibliography & 139
\end{tabular} 


\section{List of Figures}

1.1 Hypersonic flow topology. . . . . . . . . . . . . . . . . . . . . . . . 2

1.2 Examples of re-entry configurations. $\ldots \ldots \ldots \ldots \ldots \ldots \ldots$

2.1 Schematic representation of chemical reactions with a third body interaction. . . . . $\quad 9$

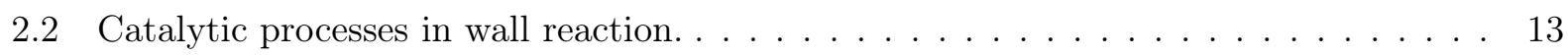

2.3 Configuration of the MESOX facility in PROMES. . . . . . . . . . . . . . . . . 15

2.4 NASA-Ames Research Center Side-Arm Reactor for catalytic characterization. . . . . 17

2.5 The NASA-Ames Aerodynamic Heating Facility (Credit: NASA). . . . . . . . . . . 18

2.6 The VKI-Plasmatron Facility. . . . . . . . . . . . . . . . . . . . . . . . . 19

2.7 The VKI-Plasmatron test chamber. . . . . . . . . . . . . . . . . . . . . . . . 20

2.8 Illustration of the LHTS concept on a Plasmatron facility. . . . . . . . . . . . . . 22

3.1 Working principles of the enthalpy probe. $\ldots \ldots \ldots \ldots \ldots \ldots \ldots$

3.2 Temperature field (in K) provided by CoolFluid of a representative Plasmatron test simulation at 50 mbar, $148 \mathrm{~kW}$ of power and $16 \mathrm{~g} / \mathrm{s}$ of mass flow. . . . . . . . . . . 28

3.3 Relation between catalysis and boundary layer outer edge enthalpy in a Plasmatron

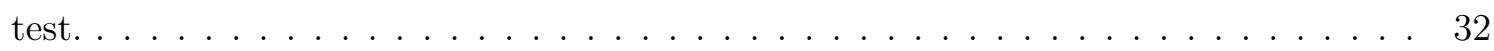

3.4 Diagram of the $H_{e}$ rebuilding method. $\ldots \ldots \ldots \ldots \ldots \ldots \ldots \ldots$

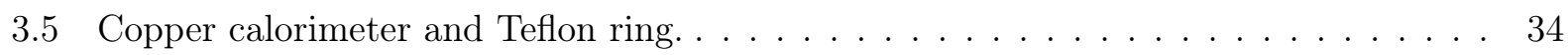

$3.6 \quad$ ESA-Standard probe with calorimeter at the center. . . . . . . . . . . . . . . . . . . . . . . . . . .

$3.7 \quad$ Illustration of Mini-Max method application. . . . . . . . . . . . . . . . . . 35

3.8 Plasmatron test configuration for $\gamma_{r e f}$ determination with the Mini-Max. . . . . . . 36

3.9 Calorimeters for Mini-Max methodology application: left - Ag, middle - Cu, right -

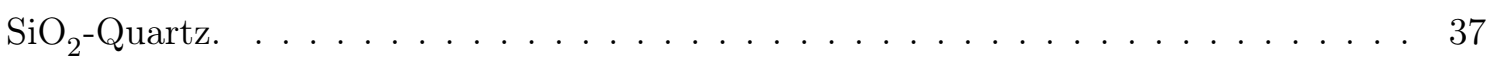

3.10 Heat flux measurements for Mini-Max application at 50 mbar. Regressions are used for data post-processing in CERBERE. . . . . . . . . . . . . . . . . . . . 39

3.11 Reference recombination coefficient at 15 mbar. . . . . . . . . . . . . . . . . . . 42

3.12 Reference recombination coefficient at 50 mbar. . . . . . . . . . . . . . . . 43

3.13 Reference recombination coefficient at 100 mbar. . . . . . . . . . . . . . . . . . . 44

3.14 Reference recombination coefficient at 200 mbar. . . . . . . . . . . . . . . . . . . 44

3.15 Reference recombination coefficient model on copper for enthalpy rebuilding in the Plasmatron. $\mathrm{A} \pm 35 \%$ of uncertainty is assumed based on preliminary UQ analysis

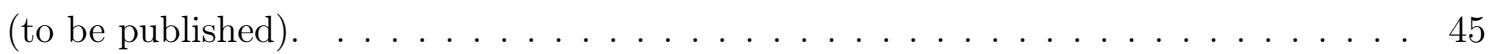

$4.1 \quad$ Static pressure effect on the $H_{e^{-}} \gamma$ relation built with CERBOULA. . . . . . . . . . . 48

4.2 Static pressure effect on the N- and O-atom profiles under non-catalytic conditions. . 48 
$4.3 \quad N u$ number as a function of $D a_{g}$ and $D a_{w}$ for $H=9 . \ldots \ldots \ldots \ldots \ldots$

$4.4 \quad N u$ number as function of $D a_{g}$ and $D a_{w}$, computed with NEBOULA. . . . . . . . 53

4.5 Relative heat flux as function of $D a_{g}$ and $\gamma$, computed with NEBOULA. . . . . . . 54

4.6 The VKI Damköhler probes. . . . . . . . . . . . . . . . . . . . 55

4.7 Example of the $\mathrm{O}$ and $\mathrm{N}$ profiles among the Damköhler probes for the same testing conditions of temperature and pressure and a non-catalytic wall. . . . . . . . . . 57

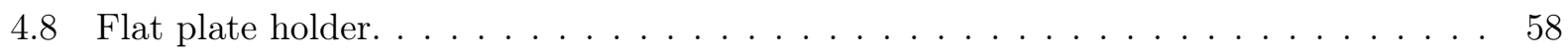

4.9 Linear calorimeter. . . . . . . . . . . . . . . . . . . . . . . . . . . 58

4.10 Details of a heat flux sensor in the linear calorimeter. . . . . . . . . . . . . . . . 59

4.11 The flat plate holder with the linear calorimeter installed. . . . . . . . . . . . . 59

4.12 Plasmatron test configuration for catalysis determination on the Damköhler probes. 60

4.13 Recombination coefficients on the Damköhler probes at 15 mbar. . . . . . . . . . . . . 64

4.14 Recombination coefficients on the Damköhler probes at 50 mbar. . . . . . . . . . . . 65

4.15 Two-dimensional map of the $N u$ number as a function of $D a_{g}$ and $D a_{w}$ for $H=9$. . 66

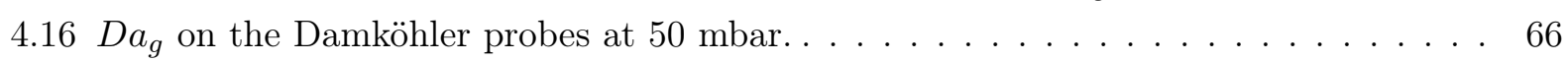

4.17 Non-dimensional numbers obtained at 50 mbar. . . . . . . . . . . . . . . . . 67

4.18 Recombination coefficients on the Damköhler probes at 100 mbar. . . . . . . . . . 68

$4.19 D a_{g}$ on the Damköhler probes at 100 mbar. . . . . . . . . . . . . . . 69

4.20 Non-dimensional numbers obtained at 100 mbar. . . . . . . . . . . . . . . . 69

4.21 Plasmatron test configuration for catalysis determination on the flat plate. . . . . . 71

4.22 Water temperature distribution along the linear calorimeter. . . . . . . . . . . . . 72

4.23 Temperature profiles generated with ICP simulations and imposed as inlet boundary condition in CFD ++ computations. . . . . . . . . . . . . . . . . . 74

4.24 Velocity profiles generated with ICP simulations and imposed as inlet boundary con-

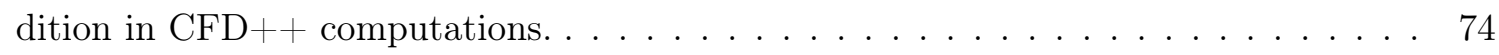

4.25 Heat flux distribution along the calorimeter at 50mbar. . . . . . . . . . . 75

4.26 Heat flux distribution along the calorimeter at 100mbar. . . . . . . . . . . . . . 75

4.27 Recombination coefficient distribution along the linear calorimeter. . . . . . . . . . 76

5.1 Local off-stagnation point boundary layer duplication concept from a flying body to

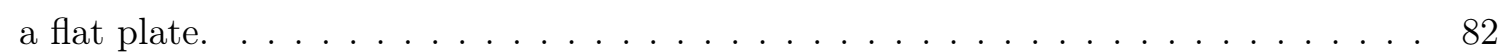

5.2 Relative differences of the boundary layer outer edge parameters on the flat plate with respect to IXV. . . . . . . . . . . . . . . . . . 86

5.3 Relative differences of the heat flux and thickness with respect to the IXV, under the

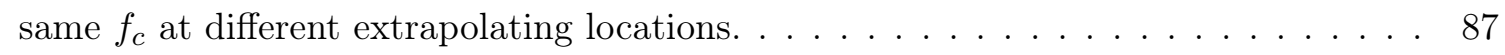

$5.4 \quad$ Relative differences of flight condition factor and thickness with respect to the IXV, under the same $\dot{q}_{w}$ at different extrapolating locations. $\ldots \ldots \ldots \ldots$. . . . . 88

5.5 Temperature profiles after the extrapolation: location (a) . . . . . . . . . . 90

5.6 Temperature profiles after the extrapolation: location (e). . . . . . . . . . . . 90

$5.7 \quad$ Velocity profiles after the extrapolation: location (a). . . . . . . . . . . . . 91

$5.8 \quad$ Velocity profiles after the extrapolation: location $(\mathrm{e}) . \ldots \ldots \ldots \ldots \ldots$. . . . . . . 91

5.9 N-atom profiles after the extrapolation: location (a). . . . . . . . . . . . . . 92 
5.10 N-atom profiles after the extrapolation: location (e). . . . . . . . . . . . . . . . 92

5.11 Interpolated inlet temperature profile from Plasmatron measurements. . . . . . . . . . 94

5.12 Interpolated inlet velocity profile from Plasmatron measurements. . . . . . . . . . . . 94

5.13 Enthalpy profiles assessment at $10 \mathrm{~cm}$ from the leading edge. . . . . . . . . . . . . 95

5.14 Enthalpy profiles assessment at $20 \mathrm{~cm}$ from the leading edge. . . . . . . . . . . . 96

5.15 Velocity variation assessment at $10 \mathrm{~cm}$ from the leading edge. . . . . . . . . . . . . 96

5.16 Velocity variation assessment at $20 \mathrm{~cm}$ from the leading edge. . . . . . . . . . . . . 97

5.17 Temperature measurements along the flat plate calorimeter. . . . . . . . . . . . . . 99

5.18 Heat flux distributions along the flat plate calorimeter. . . . . . . . . . . . . . 100

5.19 Heat flux distributions along convective times $x / U_{\infty} \ldots \ldots \ldots \ldots$. . . . . . 100

6.1 The IXV mission flight path and stages. . . . . . . . . . . . . . . . . . . . . . . . 101

6.2 Schematic illustration of CATE, with thermocouples. Rectangles numbered from 1 to 5 represent the five shingle panels at the windward side of IXV. The dimensions

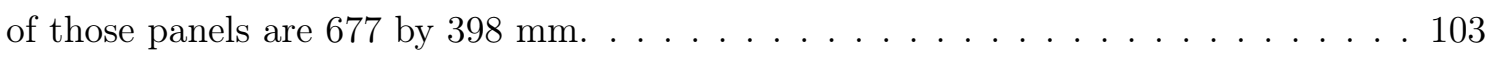

6.3 Typical SepcarbInox ${ }^{\circledR}$ CMC TPS assembly design. . . . . . . . . . . . . . . . . . 105

6.4 CMC TPS panels in hardened state: external surface (top) and internal surface

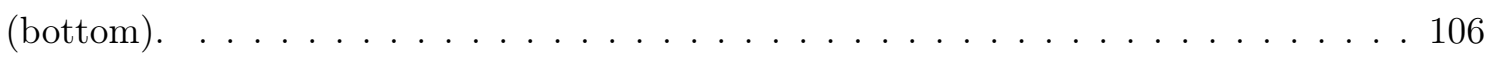

6.5 Graphite mask on shingle 5 before coating with a CMC sample attached for qualifi-

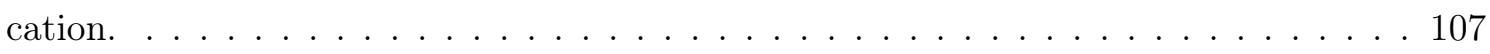

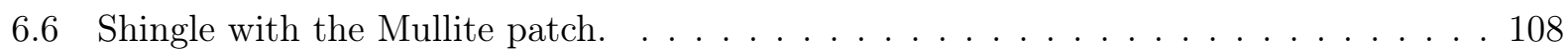

6.7 Cross-section of the VPS coating. . . . . . . . . . . . . . . . . . . . 108

6.8 Illustration of the $\mathrm{C} / \mathrm{SiC}$ bridges and thermocouple routing inside the TPS. The tape was replaced by ceramic-based glue prior to TPS assembly (photograph courtesy of TASI). . . . . . . . . . . . . . . . . . . . . . . . . 109

6.9 Final stage of IXV's assembly line (photograph courtesy of ESA). . . . . . . . . 110

6.10 Photograph of IXV after re-entry, taken at the VKI on June 2015. . . . . . . . . . . 111

6.11 Time evolution of IXV flight path and estimated stagnation point heat flux. . . . . . 112

6.12 Total enthalpy and total pressure as function of flight altitude. . . . . . . . . . . . 112

6.13 Evolution of LHTS parameters during IXV re-entry. . . . . . . . . . . . . . . 113

6.14 Time evolution of measured temperatures by thermocouples close to both low-to-high and high-to-low catalytic transitions on shingle 3. . . . . . . . . . . . . . . 114

6.15 Time evolution of measured temperatures by thermocouples close to both low-to-high and high-to-low catalytic transitions on shingle 5. . . . . . . . . . . . . 115

6.16 Time evolution of temperature jump across the two catalytic transitions in shingle 3.116

6.17 Time evolution of temperature jump across the two catalytic transitions in shingle 5.116

6.18 CATE temperature measurements at $t=4521 \mathrm{~s}$ for shingle $3 . \ldots \ldots \ldots$

6.19 CATE temperature measurements at $t=4500 \mathrm{~s}$ for shingle $5 . \ldots \ldots \ldots \ldots$ 



\section{List of Tables}

3.1 Uncertainties of measured quantities $\ldots \ldots \ldots \ldots$

3.2 Measurements of Mini-Max testing campaign at: (a) 15 mbar, (b) 50 mbar, (c) 100

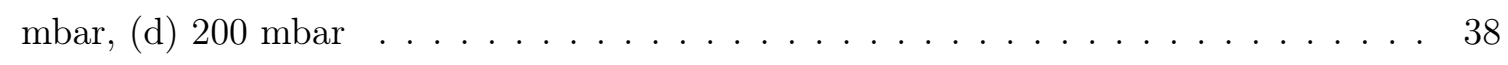

3.3 Linear interpolation coefficients for the heat fluxes measured during the Mini-Max method application $\left(\dot{q}_{c w}^{(i)}=y_{0}+m \dot{q}_{c w}^{(r e f)}\right) \ldots \ldots \ldots \ldots \ldots$

3.4 Intersections after Mini-Max application . . . . . . . . . . . . . . . . . . 40

$3.5 \quad$ Definition of $\gamma_{r e f}$ and the rebuilt $H_{e}$ after Mini-Max application $\ldots \ldots \ldots \ldots . . \ldots 4$

4.1 Dimensions of the Damköhler probes $\ldots \ldots \ldots \ldots \ldots \ldots$

4.2 Heat flux measurements during the Damköhler probes test campaign: a) 15 mbar;

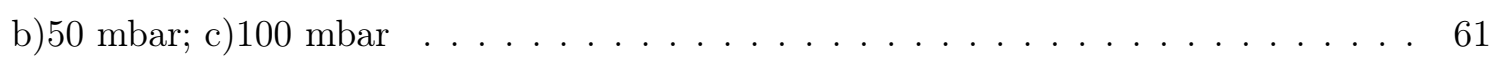

4.3 Linear interpolation coefficients for the heat fluxes measured on the Damköhler probes

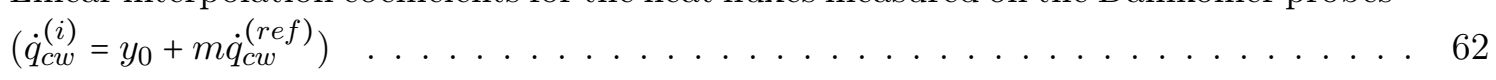

4.4 Outer edge enthalpy, recombination coefficients and boundary layer thicknesses at 15 mbar on the Damköhler probes . . . . . . . . . . . . . . . . 63

4.5 Outer edge enthalpy, recombination coefficients and boundary layer thicknesses at 50 mbar on the Damköhler probes . . . . . . . . . . . . . . . . . . 64

4.6 Outer edge enthalpy, recombination coefficients and boundary layer thicknesses at 100 mbar on the Damköhler probes $\ldots \ldots \ldots \ldots \ldots$. . . . . . . . . 68

$4.7 \quad$ Thermocouple locations from the leading edge and their time-averaged temperature

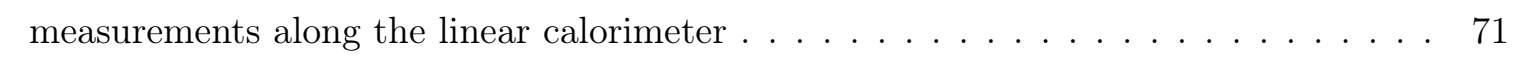

$4.8 \quad$ Regression coefficients for $T(x)=A \ln (x)+B \ldots \ldots \ldots \ldots \ldots \ldots \ldots$. . . . . . . . . . . . . . . . .

4.9 Rebuilt outer edge conditions on the Standard probe $\ldots \ldots \ldots \ldots$. . . . . . . 73

5.1 List of variables appearing in Eq. $5.3 \ldots \ldots \ldots \ldots \ldots \ldots \ldots$

5.2 List of independent variables for extrapolation $\ldots \ldots \ldots \ldots \ldots \ldots$

5.3 Boundary conditions of the IXV simulation . . . . . . . . . . . . . . . . 84

5.4 IXV simulation results at positions $(\mathrm{a})$ through $(\mathrm{e}) \ldots \ldots \ldots \ldots \ldots \ldots$

5.5 Relative difference of the output variables with respect the IXV, if $f_{c}^{(I X V)}=f_{c}^{(F P)}$. $\quad 86$

5.6 Relative difference of the output variables with respect the IXV, if $L_{F P} \approx \xi^{(I X V)} / \rho_{e} \mu_{e} u_{e} 87$

5.7 Relative difference of the output variables with respect the IXV, if $\dot{q}_{w}^{(I X V)}=\dot{q}_{w}^{(F P)} . \quad 88$

$5.8 \quad L_{F P}$ obtained using different extrapolating options (in $\mathrm{cm}$ ), from (a) to (e) $\ldots . . .89$

5.9 Relative difference of the output variables on the flat plate model with respect the

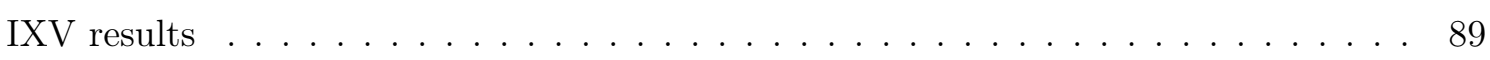

5.10 Test matrix for off-stagnation point methodology validation . . . . . . . . . . . . 97

5.11 Stagnation point measurements and rebuilt free-stream conditions . . . . . . . . . 98 
5.12 Thermocouple locations from the leading edge and their time-averaged temperature measurements along the linear calorimeter for extrapolation methodology validation

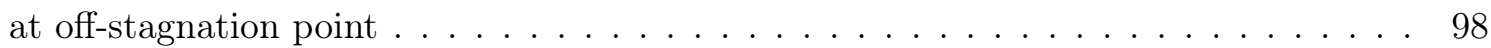

5.13 Fitting coefficients for $T(x)=A \ln (x)+B \ldots \ldots \ldots \ldots \ldots$

6.1 Thermocouples' location and designation for CATE (with $x=0$ corresponding to the base surface of IXV). ${ }^{*}$ WT51 was reported damaged during assembly . . . . . . . . . 104

6.2 Internal insulating layers on the TPS of IXV $\ldots \ldots \ldots \ldots \ldots \ldots$

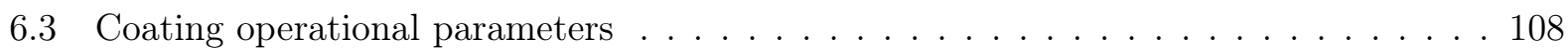

$6.4 \quad$ IXV free-stream conditions at peak heating altitude $\ldots \ldots \ldots \ldots \ldots \ldots$

6.5 LHTS parameters for IXV peak heating conditions $\ldots \ldots \ldots \ldots \ldots \ldots$

6.6 IXV free-stream conditions for $\Delta T^{(M A X)}$ over the low-to-high catalytic transition. . 115

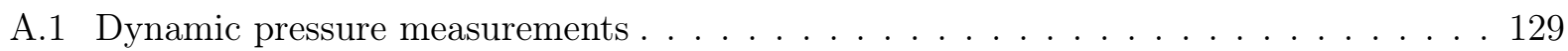

A.2 Linear interpolation coefficients for dynamic pressure measurements $\left(\dot{q}_{c w}^{(i)}=y_{0}+m \dot{q}_{c w}^{(r e f)}\right) 129$

B.1 NDP database generated with CoolFluid for Mini-Max methodology application . . . 132

B.2 Standard probe NDP database generated with CoolFluid during Damköhler probe

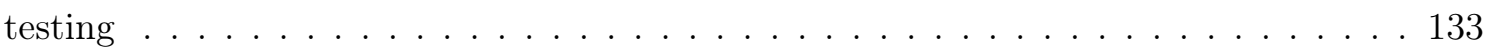

B.3 $\quad$ Frozen probe NDP database generated with CoolFluid during Damköhler probe testing 134

B.4 Equilibrium probe NDP database generated with CoolFluid during Damköhler probe

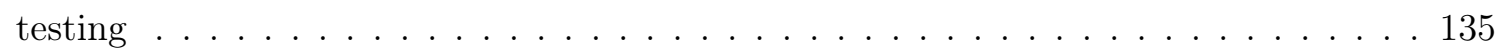

C.1 Non-dimensional parameters obtained during Damköhler probes tests at 15 mbar . . 137

C.2 Non-dimensional parameters obtained during Damköhler probes tests at $50 \mathrm{mbar}$. . 137

C.3 Non-dimensional parameters obtained during Damköhler probes tests at $100 \mathrm{mbar}$. . 138 


\section{Nomenclature}

\section{Constants}

$\begin{array}{lll}k_{B} & \text { Boltzmann's constant } & 1.380658 \times 10^{-23}\left[\mathrm{~J} \mathrm{~K}^{-1}\right] \\ \sigma & \text { Stefan-Boltzmann constant } & 5.670373 \times 10^{-8}\left[\mathrm{~W} \mathrm{~m}^{-2} \mathrm{~K}^{-4}\right] \\ R & \text { Gas constant } & 8.3144598\left[\mathrm{~J} \mathrm{~mol}^{-1} \mathrm{~K}^{-1}\right] \\ \pi & \mathrm{Pi} & 3.14159265[-]\end{array}$

\section{Roman Symbols}

\begin{tabular}{lll}
$A$ & area & {$\left[\mathrm{m}^{2}\right]$} \\
$A_{r}$ & Arrhenius pre-exponential factor & {$[-]$} \\
$\mathcal{B}$ & chemical energy accommodation coefficient & {$[-]$} \\
$c_{i}$ & species mass fraction & {$[-]$} \\
$c_{p}$ & isobaric specific heat & {$\left[\mathrm{J} \mathrm{kg}{ }^{-1} \mathrm{~K}^{-1}\right]$} \\
$d$ & width of the linear calorimeter & {$[\mathrm{m}]$} \\
$D a$ & Damköhler number & {$[-]$} \\
$D$ & species diffusion coefficient & {$\left[\mathrm{m}^{2} \mathrm{~s}^{-1}\right]$} \\
$\mathcal{D}_{i j}$ & binary diffusion coefficient & {$\left[\mathrm{m}^{2} \mathrm{~s}^{-1}\right]$} \\
$E$ & internal energy & {$\left[\mathrm{J} / \mathrm{kg}^{-1}\right.$} \\
$\mathcal{E}$ & total energy transferred to the wall & {$\left[\mathrm{J} \mathrm{kg}{ }^{-1}\right]$} \\
$E_{a}$ & activation energy & {$[\mathrm{J}]$} \\
$f_{c}$ & flight condition factor & {$\left[\mathrm{m}^{2} \mathrm{~kg}^{-1}\right]$} \\
$F_{g}$ & gravity & {$\left[\mathrm{m} \mathrm{s}^{-2}\right]$} \\
$F_{e}$ & electromagnetic acceleration & {$\left[\mathrm{m} \mathrm{s}^{-2}\right]$} \\
$H$ & chemical energy recovery factor & {$[-]$} \\
$h$ & enthalpy per unit mass & {$\left[\mathrm{J} \mathrm{kg}{ }^{-1}\right]$} \\
$H$ & Plasmatron enthalpy per unit mass & {$\left[\mathrm{J} \mathrm{kg}{ }^{-1}\right]$} \\
$h_{i}$ & species enthalpy & {$\left[\mathrm{Jkg}^{-1}\right]$} \\
$h_{f}$ & dissociation enthalpy & {$\left[\mathrm{J} \mathrm{kg}^{-1}\right]$} \\
$I$ & signal intensity & {$[-]$} \\
$J_{i}$ & species mass flux & {$\left[\mathrm{kg} \mathrm{s}^{-1} \mathrm{~m}^{-2}\right]$} \\
$k$ & reaction rate constant in the gas & {$\left[\mathrm{m}^{6} \mathrm{~mol}^{-2} \mathrm{~s}^{-1}\right]$} \\
$k_{w}$ & catalytic reaction rate constant & {$\left[\mathrm{m} \mathrm{s}^{-1}\right]$} \\
$L$ & length & {$[\mathrm{m}]^{-1}$} \\
$\dot{m}$ & mass flow & {$\left[\mathrm{kg} \mathrm{s}^{-1}\right]$} \\
& & \\
\hline & &
\end{tabular}




$\begin{array}{lll}m_{i} & \text { particle mass } & {[\mathrm{kg}]} \\ M & \text { Mach number } & {[-]} \\ M_{i} & \text { molar mass of species } i & {\left[\mathrm{~kg} \mathrm{~mol}^{-1}\right]} \\ \mathcal{M}_{i} & \text { impinging particle flux } & {\left[\mathrm{m}^{-2} \mathrm{~s}^{-1}\right]} \\ n_{i} & \text { number density } & {\left[\mathrm{m}^{-3}\right]} \\ N_{s} & \text { number of species } & {[-]} \\ N u & \text { Nusselt number } & {[-]} \\ p & \text { pressure } & {[\mathrm{Pa}]} \\ P_{s} & \text { static pressure in Plasmatron } & {[\mathrm{Pa}]} \\ P_{d y n} & \text { dynamic pressure in Plasmatron } & {[\mathrm{Pa}]} \\ P W & \text { power applied to the coil } & {[\mathrm{W}]} \\ \dot{q} & \text { heat flux } & {\left[\mathrm{W} \mathrm{m}{ }^{-2}\right]} \\ r, R & \text { radius } & {[\mathrm{m}]} \\ s & \text { wall coordinate } & {[\mathrm{m}]} \\ T & \text { temperature } & {[\mathrm{K}]} \\ u & \text { velocity parallel to the wall } & {\left[\mathrm{m} \mathrm{s}{ }^{-1}\right]} \\ v & \text { average atomic velocity at the surface } & {\left[\mathrm{m} \mathrm{s}{ }^{-1}\right]} \\ x_{i} & \text { species mole fraction } & {[-]} \\ y_{i} & \text { species mass fraction } & {[-]}\end{array}$

\section{Greek Symbols}

$\begin{array}{lll}\beta & \text { velocity gradient } & {\left[\mathrm{s}^{-1}\right]} \\ \delta & \text { boundary layer thickness } & {[\mathrm{m}]} \\ \varepsilon & \text { emissivity } & {[-]} \\ \gamma & \text { recombination coefficient } & {[-]} \\ \rho_{i} & \text { species density } & {\left[\mathrm{kg} \mathrm{m}^{-3}\right]} \\ \lambda & \text { thermal conductivity } & {\left[\mathrm{W} \mathrm{K}^{-1} \mathrm{~m}^{-1}\right]} \\ \mu & \text { dynamic viscosity } & {[\mathrm{Pa} \mathrm{s}]} \\ \nu & \text { stoichiometric coefficient } & {[-]} \\ \Pi & \text { CERBERE non-dimensional parameters } & {[-]} \\ \xi(x) & x \text {-coord. Lees-Dorodnitsyn transformation } & {\left[\mathrm{kg}^{2} \mathrm{~m}^{-2} \mathrm{~s}^{-2}\right]} \\ \hat{\eta}(x, y) & y \text {-coord. Lees-Dorodnitsyn transformation } & {[-]} \\ \tau & \text { characteristic time } & {[\mathrm{s}]} \\ \dot{\omega}_{i} & \text { species production rate } & {\left[\mathrm{kg} \mathrm{m}^{-3} \mathrm{~s}^{-1}\right]} \\ \zeta & \text { relative error between properties } & {[-]}\end{array}$

\section{Subscript}

ads relative to adsorption mechanism 


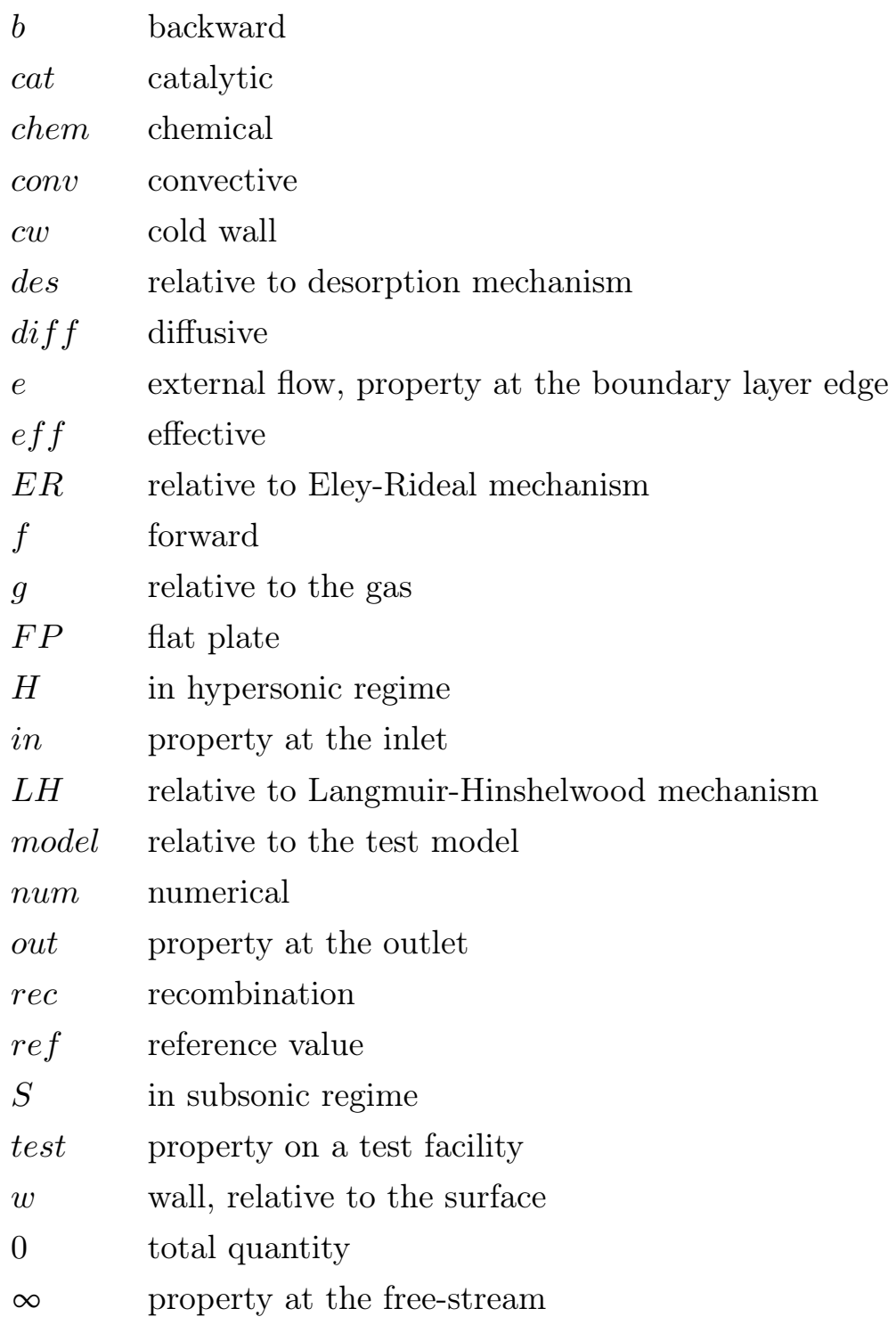

\section{Acronyms}

AHF Aerodynamic Heating Facility

CATE CATalytic Experiment

CERBERE Catalycity and Enthalpy REbuilding on REference probe

CFD Computational Fluid Dynamics

CMC Ceramic Matrix Composite

CVI Chemical Vapor Infiltration

ESA European Space Agency

FLPP Future Launchers Preparatory Program

FRC Finite Rate Chemistry

GNC Guidance Navigation and Control

GSI Gas-Surface Interaction

ICP Inductively Coupled Plasma 
IHF Interaction Heating Facility

IPG Inductive Plasma Generator

IPM

Institute of Problems in Mechanics

IXV

Intermediate eXperimental Vehicle

LEO

Low Earth Orbit

LIF

Laser Induced Fluorescence

LTE

Local Thermodynamic Equilibrium

MESOX

Moyen d'Essai Solaire d'OXidation

NEBOULA

Non-Equilibrium BOUndary LAyer

NDP

Non Dimensional Parameters

OES

Optical Emission Spectroscopy

PMT

Photo-Multiplier Tubes

REDES

Real-time Enthalpy Determination Emission Spectroscopy

SEM

Scanning Electron Microscope

TASI

Thales Alenia Space Italy

TPS

Thermal Protection System

UQ

Uncertainty Quantification

VKI

von Karman Institute for Fluid Dynamics

VPS

Vacuum Plasma Spraying

VSL

Viscous Shock Layer 


\section{Chapter 1 .}

\section{Introduction}

"Space, the final frontier". The $20^{\text {th }}$ century will always be remembered for granting access to space. During its first half, the human kind paved its way to flight. Like a child learning how to walk, early technologies in aeronautics were developed step by step. All began with the first flight of Wright brothers in 1903 and, since then, trial and error strategies were followed to break new range and speed records. There is no doubt that those accomplishments were brought by new engineering challenges in terms of aerodynamics, structures and engines, giving birth to newer technologies and possibilities. The maturity of large scale rocket engine designs in the second half of the century finally allowed a satellite to be put in orbit in 1957 for the first time in history. It was called Sputnik 1 and it set the beginning of a new era that not only defined our way of living today, but also opened the door for space exploration within the infinity of our universe.

Today, back to the $21^{\text {st }}$ century, space missions are the daily basis in our society. Satellites are constantly put in orbit for both telecommunication services and earth observation, space probes are launched for planetary exploration, and even new concepts are being considered to establish human colonies on the Moon and Mars. Because all these missions require either traveling astronomic distances or to last for several years, Earth's atmosphere becomes just a very thin layer to be crossed when jumping into space and coming back to ground. Indeed, putting a satellite in orbit or recovering a capsule from space only takes a few minutes. However, and despite the atmosphere seeming to have a small relevance in the overall mission, crossing it at high speed has certainly a significant impact on the external design of a re-entry vehicle. This dissertation is dedicated to provide understanding of one particular phenomena, and yet critical, happening during atmospheric entry.

\subsection{A picture of atmospheric entry}

Coming back from space is a challenging endeavor. Typical re-entry velocities range between 7.5 and $11 \mathrm{~km} / \mathrm{s}$ at around $100 \mathrm{~km}$ altitude. The lack of diffusive phenomena in orbit requires both kinetic and potential energies to be dissipated during the atmospheric entry while passing through all the aerodynamic regimes: from high speed hypersonics to incompressible subsonic flows. Being the former the most critical, the design of the vehicle should include both aerodynamic and structural strategies to withstand the extreme environments encountered throughout the hypersonic phase.

During the initial stage of a re-entry, the high speed flow and the low pressure environments make a strong shock wave to appear in front of the vehicle. Through this shock, temperature can increase up to $10,000 \mathrm{~K}$ and the gas can dissociate into a mixture of ionized species (plasma). This 
means that the kinetic energy of the gas surrounding the vehicle is transformed into internal energy across the shock by exciting the rotational, vibrational and electronic modes of molecules and by dissociating them into atoms through endothermic reactions. Remark that, if there were neither dissociation nor energy mode excitation in the shock, the gas temperature would be much higher [1].

Following the stagnation line, and from the shock to the flying body, one can distinguish three different regions (see Fig. 1.1):

- A shock layer where the particle system in non-equilibrium evolves towards an equilibrium temperature and composition. This means that energy levels inside molecules relax by radiating an important amount of energy.

- A Local Thermodynamic Equilibrium (LTE) zone where the Gibbs free energy reaches its minimum and the gas thermodynamic properties depend only on pressure and temperature.

- A boundary layer close to the body, confining the diffusive effects that adapt the fluid external properties to the conditions imposed by the wall. Depending on the conservation equation under consideration, one could define different types of boundary layers. For instance, species conservation equations introduce a chemical boundary layer where atoms eventually recombine into molecules through an exothermic process. Also, a viscous boundary layer grows for the diffusion of momentum, together with a thermal boundary layer appearing for temperature accommodation. The relative influence of one over the other is estimated through the nondimensional numbers: Prandtl (Pr), Lewis (Le) and Schmith (Sc). The diffusion processes taking place inside these boundary layers are responsible for the total heat flux transferred to the wall of a vehicle.

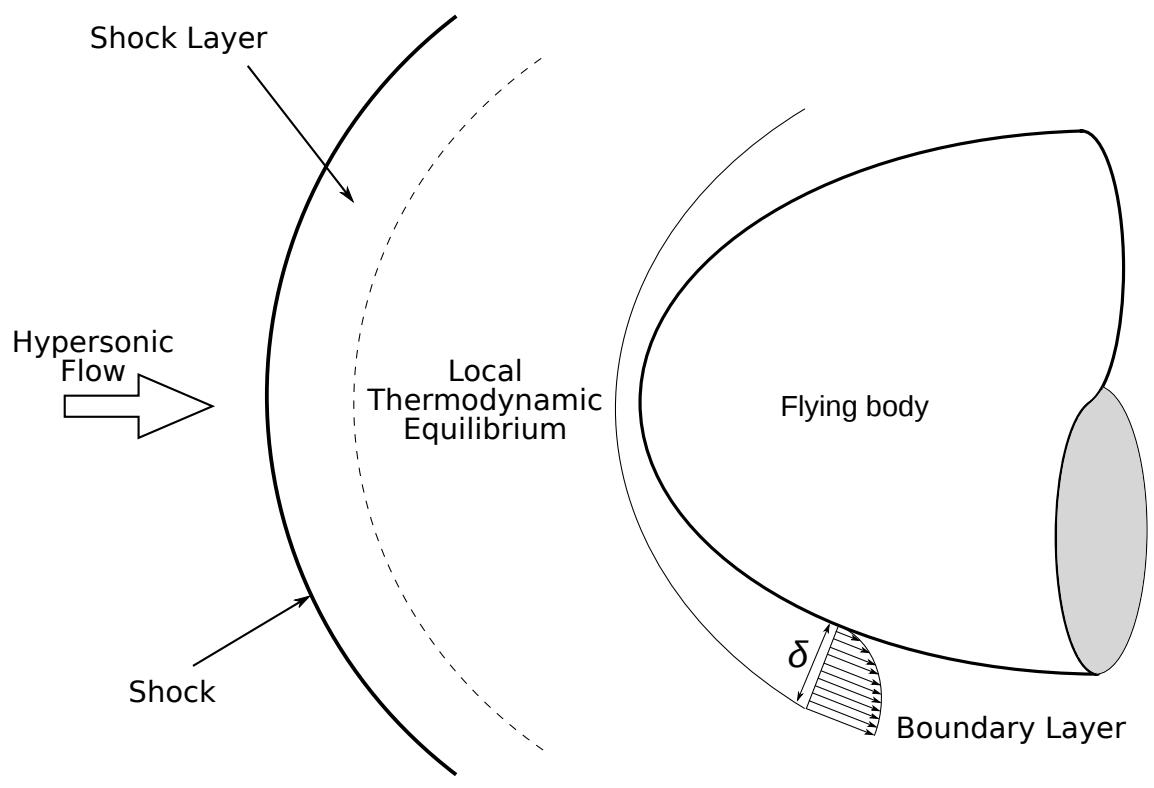

Figure 1.1.: Hypersonic flow topology.

To protect the payload in such a harsh environment, space vehicles are equipped with heat shields, commonly known as Thermal Protection Systems (TPS). There are different strategies to dissipate 
energy away from the vehicle so that thermal loads are minimized. They normally depend on the type of re-entry, and they are embedded in the spacecraft external design. The Space Shuttle is one example often used to explain that a large nose radius increases the stand-off distance between the shock layer and the wall of the body, which significantly reduces the heat flux.

The interaction between the hot gas and the TPS material is also a relevant factor to be considered for the design of the vehicle. For instance, typical heat fluxes during a fast ballistic re-entry, as in Fig. 1.2a, are much higher than the ones found in a slower lifting body configuration, such the one shown in Fig. 1.2b. Therefore, it is more efficient for the former to dissipate energy by consuming the wall of the vehicle through chemical reactions with the surrounding species using an ablative TPS, rather than withstanding the extreme temperatures using materials with higher densities. Conversely, thermal conditions are not so severe in a lifting body configuration, so non-ablating reusable TPS are used for sake of cost reduction. The chemical relations between TPS materials and gas particles are described by the so-called Gas-Surface Interaction (GSI) models. Thus, a proper understanding of GSI phenomena is necessary to accurately predict the amount of energy being exchanged under any of the situations mentioned above.

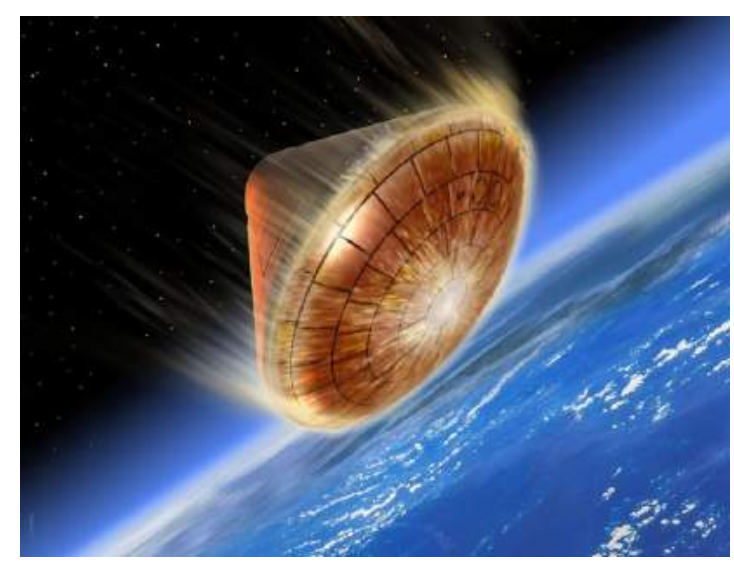

(a) Ballistic re-entry.

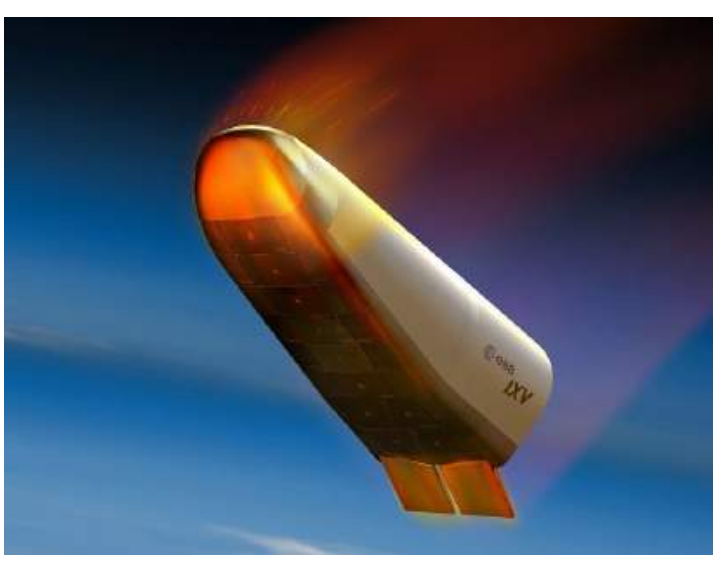

(b) Lifting re-entry.

Figure 1.2.: Examples of re-entry configurations.

This thesis is developed in the framework of lifting body re-entry flights. Therefore, the present work is focused on the modelling of catalytic reactions normally taking place on the surface of reusable TPS. Such reactions are recombination of atomic species enhanced by the material catalytic properties, which are normally characterized by the catalytic recombination coefficient $\gamma$ that will be presented in the next chapter. The heat flux at the wall $\dot{q}_{w}$ has the contributions of both convective and diffusive heatings as:

$$
\dot{q}_{w}=\dot{q}_{c o n v}+\dot{q}_{d i f f}
$$

The former is due to the thermal gradient evaluated at the wall and the latter is caused by the chemical reactions happening on the surface due to catalysis. For the success of the mission, the strategy of the TPS material is to radiate $\dot{q}_{w}$ back to the environment through a highly emissive material $(\varepsilon \approx 1)$. The radiative equilibrium condition normally used in flow solvers and, more particularly in the energy equation, is defined below for negligible thermal conduction loss inside 
the TPS:

$$
\left.\lambda \frac{\partial T}{\partial \eta}\right|_{w}+\sum_{i} J_{i, w} h_{i, w}=\varepsilon \sigma T_{w}^{4}
$$

Here, $\lambda$ is the thermal conductivity of the gas, $J_{i, w}$ is the diffusion flux of species $i, h_{i, w}$ is the mass-specific species enthalpy and $\eta$ is the coordinate perpendicular to the wall.

A TPS material characterization on ground under relevant flight conditions is required before any re-entry mission. High enthalpy facilities such as plasma wind tunnels are an invaluable asset for that purpose because they provide the post-shock thermal and chemical environments found in hypersonic regimes. This dissertation is intended to provide a better understanding about the capabilities of these facilities for catalytic characterization.

\subsection{Physical models in hypersonics}

The numerical computation of a re-entry flow environment through Computational Fluid Dynamics (CFD) is key for heat shield design and for the development of flight-to-ground extrapolation strategies. Physico-chemical models are introduced at different levels to solve the following Navier-Stokes system of equations:

$$
\begin{gathered}
\frac{\partial \rho_{i}}{\partial t}+\nabla \cdot\left(\rho_{i} \vec{V}+\vec{J}_{i}\right)=\dot{\omega}_{i} \\
\frac{\partial \rho \vec{V}}{\partial t}+\nabla \cdot(\rho \vec{V} \otimes \vec{V})+\nabla p=\nabla \cdot \overline{\bar{\tau}}+\sum_{i=1}^{N_{s}} \rho_{i}\left(\vec{F}_{g i}+\vec{F}_{e i}\right) \\
\frac{\partial \rho E}{\partial t}+\nabla \cdot[(\rho E+p) \vec{V}]-\nabla \cdot(\overline{\bar{\tau}} \cdot \vec{V})+\nabla \cdot \vec{q}=\sum_{i=11}^{N_{s}}\left(\rho_{i} \vec{V}+\vec{J}_{i}\right) \cdot\left(\vec{F}_{g i}+\vec{F}_{e i}\right)
\end{gathered}
$$

Note that the first equation of this system includes a set of mass conservation equations for each of the species $i$ present in a gas mixture. Logically, the sum among all the species equations leads to zero, meaning that no net mass is created or depleted. Because the present work is dedicated to chemical phenomena in hypersonic flows, special attention is drawn towards the species conservation equation. The total number of species in the mixture $N_{s}$ is defined by the gas model. For instance, Air-5, Air-7 and Air-11 models are typically used for Earth re-entry and define air as a mixture of 5, 7 or 11 species, respectively. Together with the gas model, chemical models are also introduced to compute the production rates $\dot{\omega}_{i}$ on the source term (right hand side) of all the species equations. Any reaction in the system can be written as:

$$
\sum_{j=1}^{N_{s}} \nu_{j r}^{\prime} X_{j} \underset{k_{b}^{r}}{\stackrel{k_{f}^{r}}{\rightleftharpoons}} \sum_{j=1}^{N_{s}} \nu_{j r}^{\prime \prime} X_{j}
$$

The corresponding reaction rate $\dot{\omega}_{i r}$ for each $r$ reaction intervening in the formation/depletion of species $i$ is normally calculated with the Law of Mass Action: 


$$
\dot{\omega}_{i r}=M_{i}\left\{\left(\nu_{i r}^{\prime \prime}-\nu_{i r}^{\prime}\right) k_{f}^{r} \prod_{j=1}^{N_{s}}\left(\frac{\rho_{j}}{M_{j}}\right)^{\nu_{j r}^{\prime}}-\left(\nu_{i r}^{\prime \prime}-\nu_{i r}^{\prime}\right) k_{b}^{r} \prod_{j=1}^{N_{s}}\left(\frac{\rho_{j}}{M_{j}}\right)^{\nu_{j r}^{\prime \prime}}\right\}
$$

which defines the production of species as a function of the reactants and products concentrations raised to the power of their stoichiometric coefficient $\nu_{j r}$ and the forward and backward reaction rate constants, $k_{f}^{r}$ and $k_{b}^{r}$, respectively. Note that the equilibrium constant $K_{r}$ for reaction $r$ is the ratio between $k_{f}^{r}$ and $k_{b}^{r}$.

Chemical models are defined with a pre-exponential factor $A_{r}$, an order $m$ and an activation energy $E_{a}$ to define the reaction rate constants as an Arrhenius type function:

$$
k_{f}^{r}=A_{r} T^{m} e^{\left(-\frac{E a}{k_{B} T}\right)}
$$

Examples of chemical models that are extensively used are Gupta et al. [2], Park [3] and Dunn and Kang [4].

Species diffusion models are also available to compute the diffusion flux $\vec{J}_{i}$ of Eq. 1.3 . The simplest examples are Fick's law for binary mixtures and its modification for multi-component mixtures as proposed by Ramshaw [5]. A more elaborate model that will be further discussed in Sec. 3.1 is the Stefan-Maxwell system of equations. Remark that the modelling of diffusive fluxes relies on kinetic theory to compute transport coefficients of mixtures, such as thermal conductivity $\lambda$, diffusion coefficient $D_{i}$ and viscosity $\mu$.

Physical models accounting for thermal non-equilibrium are sometimes used in CFD for re-entry applications [6]. This means that energy levels inside a particle do not follow a Boltzmann distribution. They are mostly used in the shock layer. Two approaches can be found in literature: the state-to-state models that describe each energy level with it's own mass conservation equation, and the multi-temperature models that define thermodynamic properties using translational, rotational, vibrational and eletronic temperatures. Although the former are more detailed and do not consider any kind of equilibrium among the energy states, they introduce additional equations into the system and they are time and resource consuming. Conversely, the latter assume thermal equilibrium within the internal modes and they add fewer equations into the system.

All the models mentioned above are implemented in CFD to solve the system of Eq. $1.3+1.5$ inside a fluid domain with a given volume. However, nothing has been said about the boundaries so far. The surface between gas and body is where the GSI models are introduced to define the boundary conditions of the system. Particularly for the species conservation equations, reaction rate constants for these models should be imposed at the wall of the vehicle. Depending on the type of TPS material, either an ablation or a catalytic model is used. The former describes the reaction between the gas and the solid taking into account surface recession, pyrolisis inside the material and reaction products being blown into the gas, while the latter considers the atomic recombination of gas particles enhanced by the surface catalytic properties. 


\subsection{Ground characterization of GSI phenomena}

The TPS material characterization in plasma wind tunnels provides data of GSI phenomena that can be used for model validation and to assist TPS design through CFD simulations. A reference flight condition should be considered when defining the test conditions on ground in order to assess the performance of a TPS material under operating conditions. Altitude and speed being the reference variables, test conditions can be defined from either a predicted trajectory during the spacecraft design phase, or an actual mission trajectory for data post-processing and analysis. In any of these situations, a flight-to-ground extrapolation strategy must be applied in order to match both spacecraft and sample environments when determining GSI modelling parameters.

The Local Heat Transfer Simulation (LHTS) concept is proposed by Kolesnikov [7, 8] to duplicate on ground the stagnation point non-equilibrium boundary layer found in flight. This would explain why most tests dedicated to assess GSI are carried out under stagnant flow configurations. LHTS states that the same boundary layer can be obtained in both flight and ground configurations if total enthalpy $h_{0}$, total pressure $p_{0}$ and external flow velocity gradient $\beta$ are respected. The logic is to define $h_{0}$ and $p_{0}$ from speed and altitude conditions in flight, and use $\beta$ to relate the nose radius and the probe geometry. The LHTS concept is adapted in the von Karman Institute for Fluid Dynamics (VKI) to subsonic plasma wind tunnels to evaluate both ablative [9] and catalytic materials [10]. The LHTS methodology will be thoroughly discussed in Sec. 2.3 .

The technique followed in VKI's Plasmatron facility for catalytic characterization of TPS material surfaces is presented by Panerai [11]. The procedure to determine the catalytic recombination coefficient $\gamma$ (to be defined in Sec. 2.1.1) relies on both intrusive measurements and numerical reconstruction of the chemically reacting environment in front of the sample through CFD solvers. Such methodology involves the free-stream enthalpy determination with a water cooled copper calorimeter exposed to the symmetry line of the plasma torch, and the proper boundary layer CFD reconstruction in front of a TPS material sample for known emissivity and surface temperature. Remark that this technique is also used at the Institute of Problems in Mechanics (IPM) in Russia using the IPG-4 [12], and both facilities can provide recombination coefficients under operating parameters representative of real flight conditions.

Panerai also provided a wide range of data for catalytic recombination coefficients in the VKIPlasmatron facility. Both metallic superalloys and ceramic TPS materials from different manufacturers were tested under several conditions of Plasmatron power, pressure and probe radius, leading to different surface temperatures on the samples. Although the catalytic boundary condition implemented in the CFD solver is only consistent with first order surface reactions, i.e. the reaction rate constant (and $\gamma$ ) only depends on surface temperature, the experimentally determined catalytic recombination coefficient seems to depend also on pressure and probe geometry. Therefore, an inconsistency seemed to appear between the modelling and the measured quantity.

Since Panerai's tests were carried out on TPS materials, any change in the Plasmatron control parameters led to a surface temperature variation on the sample, which made the influence of power, pressure and probe radius on the measured $\gamma$ difficult to assess rigorously. Part of this thesis is intended to demonstrate the effect of those parameters on the experimentally determined recombination coefficient in the Plasmatron under isothermal wall conditions. 


\subsection{Contribution of this thesis}

This thesis is developed in the framework of the European Space Agency's (ESA) Future Launchers Preparatory Program (FLPP), and more particularly, focused on the Intermediate eXperimental Vehicle (IXV) project. The IXV is a lifting-body re-entry demonstrator designed by Thales Alenia Space Italy (TASI) that aims at establishing Europe's test bed for new re-entry technologies in Guidance, Navigation and Control (GNC) systems, development of high-temperature structural materials and aerothermodynamic model validation. Launched on February 2015, it carried on board the so-called CATalytic Experiment (CATE), designed and implemented under the lead of VKI for catalytic model validation.

In this context, the objective of the present dissertation is to assess plasma wind tunnel capabilities for catalytic model development and validation. The work is intended to provide a better understanding on the behavior of the catalytic recombination coefficient measured in the Plasmatron facility, so that the recombination coefficient on IXV can be confidently determined via future ground testing using data provided by CATE. Given the fact that the flight experiment is installed on the windward side of IXV, downstream the stagnation point, the goal of this research is to provide an answer to the following question:

Can a constant recombination coefficient determined in ground testing facilities be applied along the wall of a vehicle?

Note that a constant $\gamma$ along the vehicle's wall coordinate is imposed in most commercial CFD solvers. However, Panerai's [11] results suggested that this could be a limiting assumption due to the dependence of catalytic processes with pressure and/or probe geometries. Therefore, relevant parameters found in non-equilibrium boundary layers with an influence on the experimentally determined recombination coefficient should be identified.

To accomplish such objective, a review of different approaches to model catalysis is provided in Ch. 2. The work is complemented with a description of different plasma facilities used to measure recombination coefficients on TPS material samples. The flight-to-ground extrapolation methodology for non-equilibrium boundary layer duplication at stagnation point proposed by the LHTS, which in the author's opinion should be systematically applied to relate the catalytic conditions on both ground and flight, is also explained.

In addition, two testing campaigns on water-cooled copper calorimeters are also provided in this thesis. They are intended to evaluate the influence of LHTS parameters $h_{0}, p_{0}$ and $\beta$ on the experimentally determined recombination coefficient. Despite copper not being a TPS material, the reason for proceeding with such material lies on the fact that, since it is water-cooled and its thermal conductivity is very high, its surface temperature remains constant at any test condition or probe configuration.

The first campaign is carried out in Ch. 3, on the ESA-Standard probe, and it is used to build a reference catalytic model for enthalpy characterization of the Plasmatron facility. Note that knowing the enthalpy of the plasma jet is the first step to properly determine the recombination coefficient on a TPS sample. The second test campaign, provided in Ch. 4, assesses the influence of 
the velocity gradient on the recombination coefficient by measuring $\gamma$ on different probe sizes and configurations, at both stagnation and off-stagnation point.

Furthermore, the fact that test results demonstrate an influence of the whole boundary layer diffusive properties on $\gamma$ encourages the development of a flight extrapolation strategy downstream the stagnation point. Thus, a new off-stagnation point extrapolation methodology is proposed in Ch. 5. providing a reasonable procedure to extract the recombination coefficient from IXV's windward side. Obviously, this will contribute to the post-flight activities of CATE in a near future.

Finally, select of IXV's flight data are provided in Ch. 6. They show the success of CATE for catalytic phenomena visualization. A preliminary analysis is carried out to define the most relevant flight conditions to be reproduced on ground: at stagnation point under peak heating conditions, and at off-stagnation point in the environments of CATE. 


\section{Chapter 2.}

\section{State of the Art on Catalysis}

Any type of reaction happens through collision between particles. To understand what catalysis is, it is important to consider that the presence of a third body is required in many of the chemical processes described by the Law of Mass Action written in Eq. 1.7. During a recombination process of two atoms into a molecule, the third body could be either another gas particle if the process is a volume reaction, as seen in Fig. 2.1a, or the wall itself for a surface reaction, as represented by Fig. 2.1b. As the third body remains unchanged, it is named a catalyst of the process. Surface catalysis is nothing else than a quantification of the wall microscopic properties that enhance recombination while acting as a third body in the reaction mechanism.

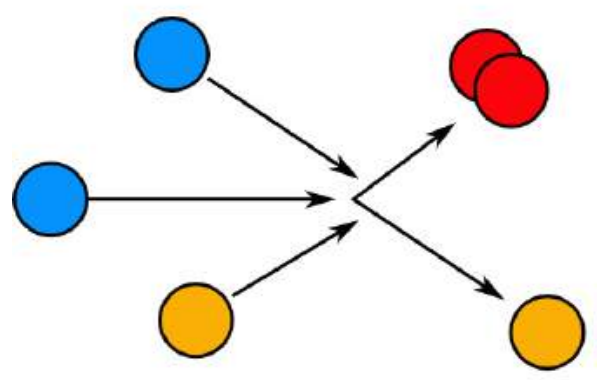

(a) Volume reaction.

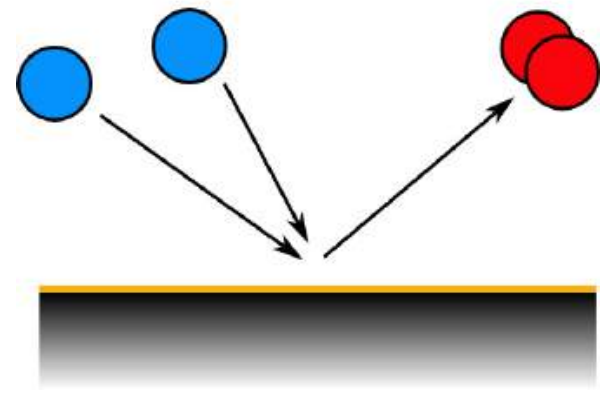

(b) Surface reaction.

Figure 2.1.: Schematic representation of chemical reactions with a third body interaction.

Remark that, when both catalyst and reactants are in the same phase, the term of homogeneous catalysis is normally used. Conversely, when the catalyst and the reactants are in different phases, the phenomenon is named heterogeneous catalysis. In this dissertation, following the common practice in the literature dealing with gaseous reactants around a solid catalyst, the general term of catalysis is used to refer to heterogeneous catalysis.

This chapter gives an overview of different strategies to model catalysis, and the techniques followed in different types of plasma facilities to measure it.

\subsection{On catalytic modelling}

Catalytic production and depletion rates $\dot{\omega}_{c a t}$ are described as a function of three variables: partial density of reactants $\rho_{i}=\rho c_{i}$, reaction order $n$ and reaction rate constant $k_{w}$, as: 


$$
\dot{\omega}_{c a t}=k_{w}\left(\rho c_{i}\right)^{n}
$$

Remark that the backward reaction rate constant that appears in Eq. 1.7 is normally neglected in the production rate for catalytic reactions [13, 14] because one could assume $k_{w}=k_{f}^{r}>>k_{b}^{r}$ at typical wall temperatures found in re-entry $(1500-2000 \mathrm{~K})$. Note also that units of $k_{w}$ vary depending on the reaction order. For instance, it is expressed in $\mathrm{m} / \mathrm{s}$ for first order reactions $(n=1)$. Mass conservation at the surface is imposed by the following boundary condition:

$$
J_{i, w}=\dot{\omega}_{c a t}
$$

which states that the species diffusion flux $J_{i, w}$ at the wall should balance the total production/depletion rates due to the wall catalytic properties.

The catalytic modelling consists on providing the rate constants $k_{w}$ for the recombining reactions happening at the surface. There are different, and yet equivalent, ways to express the catalytic reaction rate constants depending on the level of approximation. The simplest one is through the catalytic recombination coefficient $\gamma$ under the frame of a macroscopic description of the GSI phenomena, while the modelling of $k_{w}$ through several reaction mechanisms is seen as a microscopic description of catalysis.

\subsubsection{The macroscopic description}

Goulard was the first author to introduce catalysis in the aerospace field in 1958 [15]. He introduced a simple catalytic model that described the reaction rate with only one parameter: the recombination coefficient $\gamma$, also known as recombination probability, or catalycity. This parameter is defined as the ratio of the number of atoms recombining on a surface per unit area and time to the total number of atoms reaching the surface per unit area and time. It is used to compute the reaction rate constant $k_{w}$ with:

$$
\gamma=k_{w} \sqrt{\frac{2 \pi m}{k_{B} T_{w}}}
$$

A finite recombination rate implies Neumann's wall boundary condition for the species conservation equations. In his work, Goulard used Fick's diffusion model for binary mixtures:

$$
J_{i, w}=\left.\rho D \frac{\partial c_{i}}{\partial \eta}\right|_{w}=k_{w} \rho c_{i}
$$

Note that the recombination coefficient ranges between 0 and 1. The extreme assumptions of non-catalytic $(\gamma \rightarrow 0)$ and full-catalytic $(\gamma \rightarrow 1)$ were taken by Lees [16], Fay \& Riddell [17] and Fay \& Kemp [18] to solve non-equilibrium boundary layer equations. It is worth remarking that the conservative assumption of $\gamma=1$ was introduced by imposing the Dirichlet boundary condition of zero atomic concentration at the wall $\left(c_{i}=0\right)$, which is not true since the recombination level can never exceed the equilibrium composition (if so, $k_{w} \rightarrow \infty$ ). This means that the full-catalytic assumption should be seen as the condition that imposes a wall in chemical equilibrium, instead of the full recombination of all the atomic species. The heat flux variation between the two situations 
might be negligible because the amount of atoms in equilibrium at typical surface temperature is expected to be very low, but it is important to highlight the conceptual difference.

Some assumptions were introduced in Goulard's work, and they need to be taken into account for the development of this dissertation:

- He considered the gas as a binary mixture and without chemical reactions $\left(\dot{\omega}_{i}=0\right.$ in Eq. 1.3$)$. This means that the boundary layer is chemically frozen and that species diffusion is caused by surface reactions only. As it will be discussed in Ch. 4. a frozen regime is the most adequate condition for catalytic determination in plasma facilities. This fact was already pointed out by Goulard himself, quoting: "Although catalytic recombination can be included in the equilibrium flow solution, the high wall temperatures required for its effects to be appreciable are not practical, and this case is not considered here". This issue was also mentioned by Vasil'evskii et al. [19] as a limitation of plasma wind tunnels for measuring the recombination coefficient. The problem is that plasma wind tunnels, designed to reproduce re-entry environments, are not always capable to provide frozen regimes. This is the reason why part of this dissertation is dedicated to assess the influence of chemical non-equilibrium for catalytic determination in the VKI-Plasmatron facility.

- In addition, the first order assumption is made to derive Eq.2.3. This implies that the catalytic reaction rate constant $k_{w}$ must depend on wall temperature only, and that any dependence observed on other thermodynamic parameters is consequence of a reaction order higher than one $(n>1)$.

- Furthermore, he considered the gas as a binary mixture, which implies that only catalytic reactions of the kind $2 \mathrm{X} \longrightarrow \mathrm{X}_{2}$ can be considered on the surface and that the formation of NO should be neglected for air mixtures. This hypothesis was proven false on Quartz by Copeland et al. 20] who observed catalyzed NO formation in a diffusion reactor. Also, the diffusion flux described by Fick's law as in Eq. 2.4 becomes more complex for multi-component mixtures to ensure the species conservation as explained by Ramshaw [5], Scott [21] and Barbante [22], so the diffusion model should be also consistent with the catalytic condition at the wall.

More detailed $\gamma$-models can be found in literature. Sometimes it is considered that what is experimentally measured is an effective recombination coefficient instead of the real one:

$$
\gamma_{e f f}=\mathcal{B} \gamma
$$

This effectiveness may include two effects. On one hand, it is possible that not all the energy released during a catalytic reaction is transferred to the wall, which introduces the so-called energy accommodation coefficient $\mathcal{B}$ defined as:

$$
\mathcal{B}=\frac{\mathcal{E}}{\Delta h_{f}}
$$

where $\mathcal{E}$ is the total energy transferred to the wall and $\Delta h_{f}$ the dissociation energy of the recombined molecule. Models of the accommodation coefficient can be found in literature [23 25]. On 
the other hand, the roughness of the surface increases the wet area on which the atomic species can collide and recombine. This last effect was proposed by Kim et al. [26] in 1991 and more recently studied by Thoemel et al. [27, 28].

The $\gamma$-model is hereby considered as a macroscopic description because after all the assumptions postulated above, there is no little point in treating $\gamma$ as a purely chemical factor. It should instead be taken as a parameter that allows engineers to close the boundary condition of the species conservation equations according to what is extracted from experiments. For instance, when testing in the VKI-Plasmatron facility, the assumptions of $\gamma_{\mathrm{N}}=\gamma_{\mathrm{O}}$ and $\gamma_{\mathrm{NO}}=0$ need to be made because of the lack of capability to distinguish between nitrogen and oxygen recombination in air with only a heat flux measurement. Ideally, new spectroscopic methods such Laser Induced Fluorescence (LIF) could determine the species gradients of both N- and O-atoms [29], but their application is limited to frozen regimes and the technology has not been implemented in large plasma wind tunnels yet.

\subsubsection{The microscopic description}

There are more complex models describing heterogeneous catalysis. They are known as Finite Rate Chemistry (FRC) models and provide a microscopic description of the phenomena happening on a surface. Many FRC models can be found in literature. Most of them describe the global catalytic reaction by means of different recombination mechanisms, related to different rate constants: $k_{a d s}$, $k_{E R}, k_{L H}, k_{d e s}$. The relevance of each process depends on wall temperature and pressure, establishing different levels of catalysis. Some of the typical processes normally considered in these models are listed below and shown in Fig. 2.2 .

- Diffusion of atoms towards the wall.

- Chemical Adsorption $\left(k_{a d s}\right)$ : Atoms X adiabatically attach to a surface site $[\mathrm{s}]$ through a chemical bond. Adsorbed atoms are known as adatoms $\mathrm{X}_{\mathrm{s}}$.

$$
\mathrm{X}+[\mathrm{s}] \stackrel{k_{a d s}}{\longrightarrow} \mathrm{X}_{\mathrm{S}}
$$

- Eley-Rideal Mechanism $\left(k_{E R}\right)$ : A gas-phase $\mathrm{Y}$ atom reacts with an adatom $\mathrm{X}_{\mathrm{s}}$ to form a molecule XY. The mechanism is first order, and takes place at relatively low temperatures and high pressures, when there are lots of adatoms spread over the surface and the number of free sites is low. It is related to low catalytic conditions and it contributes to the chemical energy transferred to the wall.

$$
\mathrm{Y}+\mathrm{X}_{\mathrm{S}} \stackrel{k_{E R}}{\longrightarrow} \mathrm{XY}
$$

- Langmuir-Hinshelwood Mechanism $\left(k_{L H}\right)$ : This is a second order mechanism. At higher temperatures and lower pressures, catalysis increases because adatoms can diffuse more easily throughout the surface until they react with another adatom, reacting and leaving the surface as a molecule. This mechanism also contributes to the total energy transfer during the catalytic process. 


$$
\mathrm{Y}_{\mathrm{S}}+\mathrm{X}_{\mathrm{S}} \stackrel{k_{L H}}{\longrightarrow} \mathrm{XY}
$$

- Thermal Desorption $\left(k_{d e s}\right)$ : It takes place at very high surface temperatures. Adatoms have enough energy to break the chemical bond with the surface and leave it in the form of an atom. No energy is transmitted towards the wall. Therefore, as the adsorption mechanism, thermal desorption is also adiabatic.

$$
\mathrm{X}_{\mathrm{S}} \stackrel{k_{d e s}}{\longrightarrow} \mathrm{X}+[\mathrm{s}]
$$

- Diffusion of molecules away from the wall.

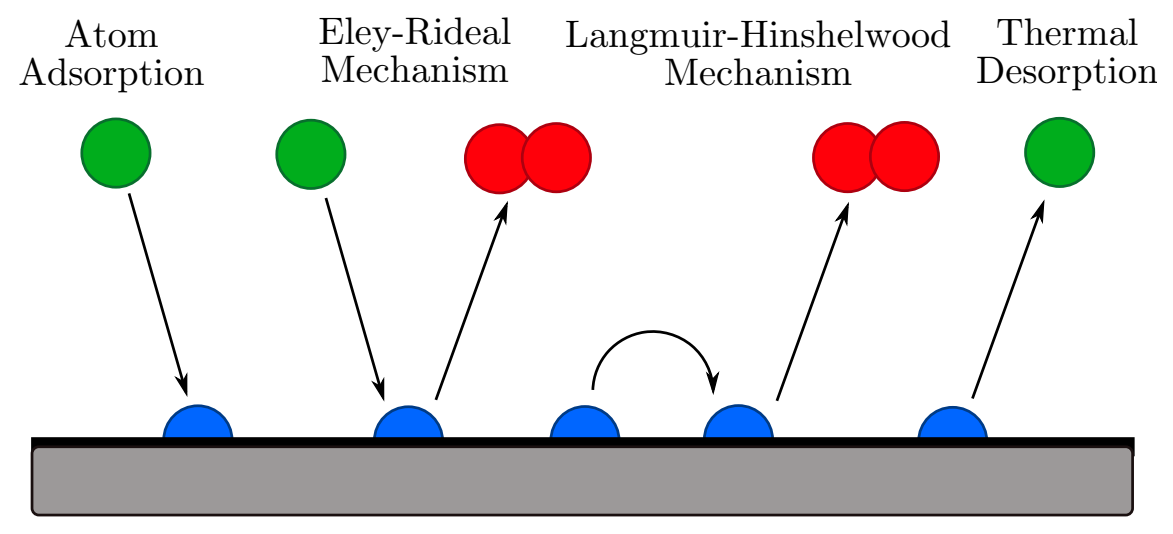

Figure 2.2.: Catalytic processes in wall reaction.

The FRC modelling consists on defining each rate constant as an Arrhenius type function (see Eq. 1.8), where the pre-exponential factor $A_{r}$ is a function of different surface parameters. The recombination coefficient is normally computed imposing a steady-state balance of the total number of adatoms at the wall. Therefore, in contrast to $\gamma$-models, the recombination coefficient is an output parameter in FRC models.

Considering literature, it is worth to remark the work of Seward et al. [30], who proposed a description for adsorption, Eley-Rideal recombination and thermal desorption processes. Recombination coefficient values predicted by his model where compared with data measured by Greaves \& Linnett [31] in 1959 on Silica. After Seward's contribution, new parameters such as surface coverage and sticking coefficient were included, among others, to model catalysis. They are extensively used in current models. For instance, Nasuti et al. 32] in 1996 and Barbato et al. 33] in 2000 considered all four wall processes for each reaction, and they proposed to express $\dot{\omega}_{c a t}$ as an Arrhenius function where activation energies $E_{a}$ had also to be defined. After that, in 2006, Armenise et al. 34] extended the modelling by considering non-Boltzmann behavior on the vibrational distribution functions near the surface. Note that historical evolution of FRC models shows that they tend to grow in complexity at a much faster rate than measurement techniques for their validation in plasma facilities. It was not until 2010 that Di Benedetto and Bruno [35] suggested that, indeed, a model simplification might be convenient for further experimental validation. 
The main advantage of FRC models is the fact that they can avoid the first order reaction hypothesis made by the $\gamma$-models through the Langmuir-Hinshelwood mechanism. This means that some pressure effects can be expected on the computation of the recombination coefficient, as shown by Marschall \& MacLean [36, 37, Fertig et al. [38, 39] and Sorensen et al. [40]. On the experimental side, Massuti-Ballester in Ref. [41], and the author himself in Ref. [42], recently suggested that pressure effects observed on their recombination coefficient measurements in a plasma wind tunnel could be consequence of the predominance of the Langmuir-Hinshelwood mechanism over the Eley-Rideal process.

\subsection{On facilities for surface catalysis determination}

Experimental validation of catalytic models requires facilities capable of measuring the variables involved. Different types of plasma facilities are available to determine the recombination coefficient on the surface of a sample, each of them with a specific measurement technique. Diffusion tubes are dedicated facilities to study both oxidation and catalytic properties of materials, while plasma wind tunnels extend their capabilities toward the whole reacting boundary layer reproduction for GSI assessment under operating conditions that are closer to the flight. Although flow configurations are different, both types of facilities are extensively used for catalytic characterization and quantification.

\subsubsection{Diffusion tubes}

The main characteristic of diffusion tubes is that the motion of the gas particles is driven by species concentration gradients between the main flow and the sample surface. If thermal diffusion is neglected and volume reactions are not taking place, i.e. the flow is chemically frozen (typically at very low pressures), species diffusion is caused by both oxidation and catalytic recombination on the sample. This means that surface chemical properties can be determined with spectroscopic flow diagnostic techniques. Four parts can be distinguished in diffusion tube designs: a plasma generator that dissociates the gas, a system to control the surface temperature, a system to monitor the species around the sample, and a non-catalytic Quartz tube inside which atoms diffuse towards the test specimen.

\subsubsection{The MESOX facility}

The Oxidation Solar Test Facility named Moyen d'Essai Solaire d'OXidation (MESOX), located in the laboratories of PROcédés, Matériaux et Energie Solaire (PROMES), is a diffusion tube in which both oxidation and surface recombination studies are performed. The facility consists of a 50 $\mathrm{cm}$ long by $5 \mathrm{~cm}$ diameter Quartz reactor placed at the focus of a $6 \mathrm{~kW}$ solar furnace. A $25 \mathrm{~mm}$ in diameter by $3 \mathrm{~mm}$ in thickness sample is normally introduced for material characterization. Both sample and reactor are placed in stagnation point configuration at the center of a refrigerated wave guide as seen in Fig. 2.3. A variable opening shutter allows the regulation of the total incident solar flux up to $4.5 \mathrm{MW} / \mathrm{m}^{2}$, and hence the surface temperature. A system of rotating and fixed mirrors 
allows monitoring the surface temperature at both the front and the back of a sample with a single pyrometer.

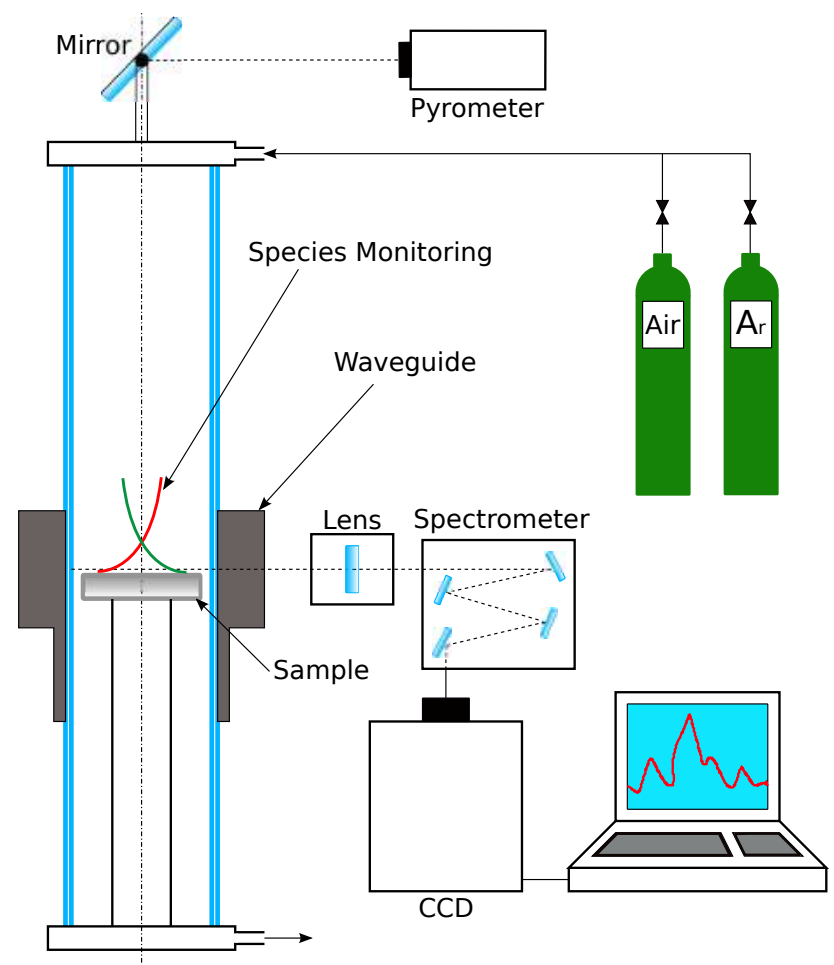

Figure 2.3.: Configuration of the MESOX facility in PROMES.

One method to quantify the catalytic activity of the material is based on an energy balance over the sample, where the diffusive heat flux is estimated using wall temperature measurements on both front and back sample surfaces, under air and argon plasma with:

$$
\left(\mathcal{B} \dot{q}_{r e c}\right)=\varepsilon \sigma\left[\left(T_{f}^{4}\right)^{\mathrm{Air}}-\left(T_{f}^{4}\right)^{\mathrm{Ar}}+\left(T_{b}^{4}\right)^{\mathrm{Air}}-\left(T_{b}^{4}\right)^{\mathrm{Ar}}\right]
$$

The incident solar flux is the same under both gases, energy loses on the sides of the sample are normally neglected and the microwave-material interaction is assumed to be equal in both air and argon, as explained by Balat et al. [43,45].

Another technique to determine catalysis is based on the monitoring of the O-atom concentration gradient around the sample through actinometry, allowing the measurement of the recombination coefficient $\gamma$. To apply this method, a non-reactive gas (actinometer) such argon, is introduced with the air mixture. The quantity of argon introduced should be optimal (around 5\%) for acquiring a good signal while having no influence on the chemical and diffusive processes. The reactor is equipped with $\mathrm{CaF}_{2}$ viewing ports that provide visual access to the flow around the sample. A spectrometer is aligned to record O-atom and Ar concentrations in the plasma mixture at different locations whithin the boundary layer. With the ratio between O-line intensity $I_{\mathrm{O}}(844.6 \mathrm{~nm})$ and Ar-line intensity $I_{\mathrm{Ar}}(842.4 \mathrm{~nm})$ being proportional to the species concentration, the recombination coefficient $\gamma$ is determined as: 


$$
\gamma=\frac{4 D_{\mathrm{O}, \mathrm{Air}}}{v \delta}\left(\frac{\left.\frac{I_{\mathrm{O}}}{I_{\mathrm{Ar}}}\right|_{e}}{\left.\frac{I_{\mathrm{O}}}{I_{\mathrm{Ar}}}\right|_{w}} \frac{T_{w}}{T_{e}}-1\right)
$$

where $D_{\mathrm{O} \text {,Air }}$ is the diffusion coefficient of O-atoms in the air mixture, $\delta$ is the boundary layer thickness, $v=\sqrt{8 R T_{w} / \pi M}$ is the average velocity of atoms given by kinetic theory and the subindices $e$ and $w$ indicate the property evaluated at the boundary layer edge and at the sample surface, respectively.

Typical materials used for space re-entry have been tested in this facility and a large database is available in literature at 200 and $300 \mathrm{~Pa}$. For instance, in 1997 Balat et al. [43] provided recombination coefficient measurements for $\mathrm{SiC}, \mathrm{SiC}+\mathrm{SiO}_{2}$ and sintered $\mathrm{Al}_{2} \mathrm{O}_{3}$. An extensive database for $\mathrm{SiO}_{2}$ ( $\beta$-cristobalite) and $\mathrm{SiO}_{2}$ (Quartz) was published in 2003 by the same author [46] and used for validation of a catlytic model in 2005 by Bedra et al. [47]. Catalysis data on different sintered $\mathrm{Al}_{2} \mathrm{O}_{3}$ samples can also be found in Ref. [48] (2007).

It is worth to mention the existence of a similar facility in Laboratoire de Génie des Procédés Plasma, École Nationale Supérieure de Chimie de Paris, in which the heating of the sample is carried out using electrical pulses instead of solar radiation. The recombination coefficient is also determined with actinometry, and assuming a linear distribution of the O-atom concentrations profile through the boundary layer thickness to calculate the atom concentration at the wall, following:

$$
\gamma=-\frac{4 D_{\mathrm{O}, \mathrm{Air}}}{v}\left(\frac{\nabla\left(\frac{I_{\mathrm{O}}}{I_{\mathrm{Ar}}}\right)}{\frac{I_{\mathrm{O}}}{I_{\mathrm{Ar}}}-\nabla\left(\frac{I_{\mathrm{O}}}{I_{\mathrm{Ar}}}\right) \delta}\right)
$$

Recombination coefficient data was reported by Nguyen-Xuan et al. [49] in 1994 and chemical energy accommodation coefficient data was published by Cauquot et al. [50] in 1998.

\subsubsection{The NASA-Ames Side-Arm reactor}

It was conceived to study diffusion-reaction processes for re-entry applications. It consists of a main tube made of glass where molecules are dissociated into atoms through a microwave discharge and a side-arm Quartz tube surrounded by a $47 \mathrm{~cm}$ metallic clam shell that allows rising the temperature of the sample. Gas inlets are located before and after the microwave reactor, while test samples are placed on the side-arm as shown in Fig. 2.4. Both tubes are connected to vacuum pumps in the gas outlet. Two valves allow changing the working configuration of the facility between flow tube and diffusion tube modes.

Species monitoring in the side-arm is carried out through a LIF technique. This methodology is based on the excitation of specific atomic energy levels using a laser beam and detecting the emitted energy during the relaxation process. A laser and a system of lenses is used to excite atoms at the appropriate wavelength and a PhotoMultiplier Tube (PMT) allows the detection of the atomic reemitted energy for a specific transition. Four PMT are used to obtain spatial resolution of the species throughout the facility. The feasibility of this method for surface recombination coefficient quantification was demonstrated by Pallix et al. [51] in 1996, under the assumption of first order reaction and the absence of volume recombination, estimating the recombination coefficient as: 


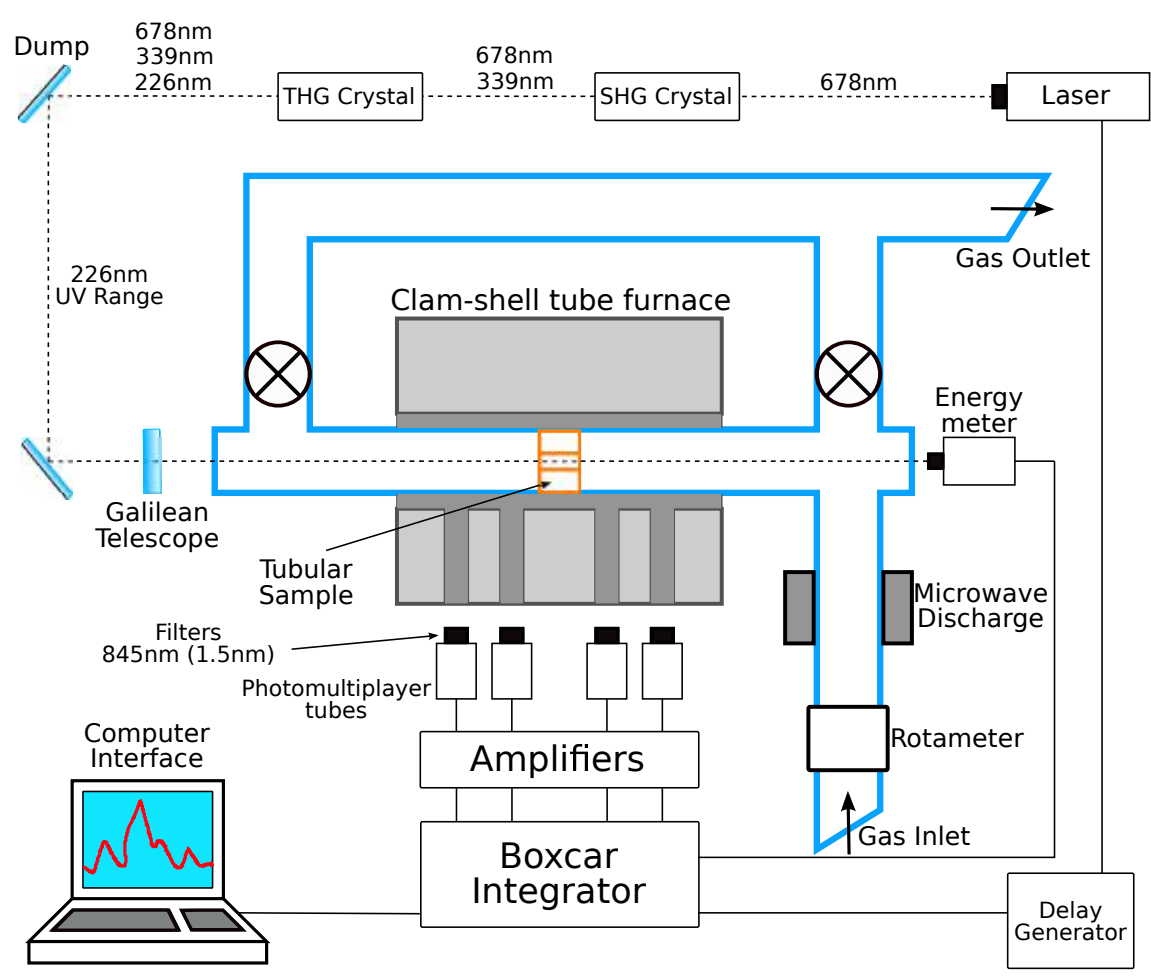

Figure 2.4.: NASA-Ames Research Center Side-Arm Reactor for catalytic characterization.

$$
\gamma=\frac{2 \mu^{2} D}{v r}
$$

where $D$ is the diffusion coefficient, $v$ is the average speed of the atoms previously defined in Sec. 2.2.1.1, $r$ is internal radius of the diffusion tube and $\mu$ is the slope of atomic decay along the tube due to catalytic reactions following:

$$
\ln \left(\frac{c(x)}{c_{0}}\right)=-\mu x
$$

Dedicated work for surface recombination measurement was published by Stewart in 1997 [52]. He combined diffusion tube and arc-jet facility measurements for a wide range of surface temperatures. The work of Copeland et al. [20] in 1998 that demonstrates the NO formation as a surface catalyzed process also deserves mention. The same method was used by Sepka et al. [53] in 2000 for surface reaction investigations in CO mixtures for Mars entry. Currently, recombination coefficients are estimated by fitting the experimental concentration profiles with CFD results based on a 2D-axisymmetric reaction-diffusion model, as shown by Marschall et al. in 2006 [54, 55].

\subsubsection{Plasma wind tunnels}

Plasma wind tunnels are mainly designed to reproduce the reacting boundary layer during the reentry. They are extensively used for TPS material qualification under relevant flight conditions. While ablative materials are normally tested in these facilities, they also offer a wide range of testing capabilities for studying oxidation and catalysis. Unlike diffusion tubes, both convective and diffusive aspects of the flow in front of a sample should be taken into account. The boundary 
layer around the sample is the region where diffusive properties are relevant for material testing.

Depending on the method to generate the plasma, one can distinguish between arc-jet facilities and Inductively Coupled Plasma (ICP) facilities. Plasma is generated with an electric discharge between two electrodes in the former, and through an electromagnetic field inside a coil that rises gas temperature by Joule effect in the latter. The next sections are dedicated to review the Aerodynamic Heating Facility (AHF) at NASA, and the VKI-Plasmatron as examples of both arc-jet and ICP facilities, respectively.

\subsubsection{The Aerodynamic Heating Facility (AHF)}

This is an arc-jet plasma wind tunnel located at NASA Ames Research Center. The main components of the facility are the heater, the nozzle and the test chamber. The heater generates plasma through an electric discharge between two electrodes. It can operate at $20 \mathrm{MW}$ under reservoir pressure ranging from 1 to $40 \mathrm{~atm}$, and enthalpies between 3.5 and $9.5 \mathrm{MJ} / \mathrm{kg}$. The heater shown in Fig $2.5 \mathrm{a}$ is coupled with a set of conical nozzles that expand the flow and define the thermodynamic conditions around the model. The test chamber contains a 5-arm injection system that allows testing 5 probes in one run. The maximum size of the samples is $35.6 \mathrm{~cm}$, and heat fluxes can reach $3 \mathrm{MW} / \mathrm{m}^{2}$ for a $10.2 \mathrm{~cm}$ hemispherical probe. A full scale SPRITE test can be seen in Fig. 2.5b. Catalytic measurements at high surface temperatures are presented by Stewart [52].

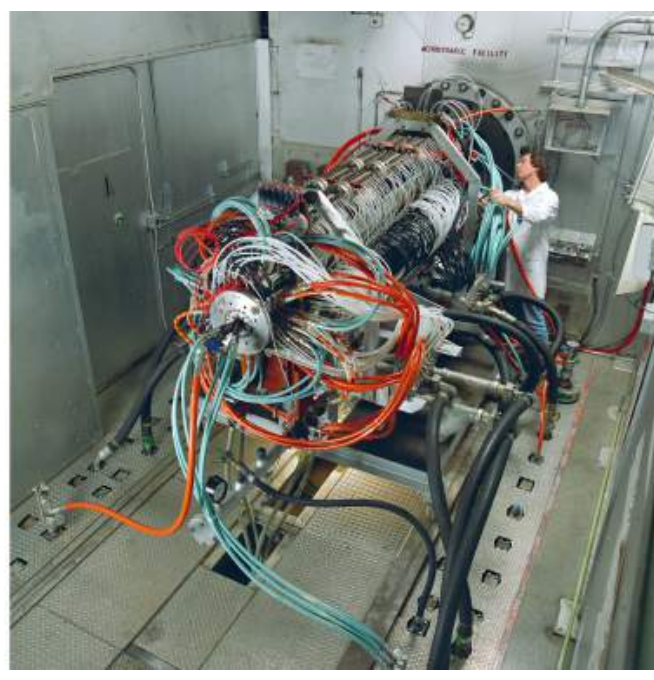

(a) The heater.

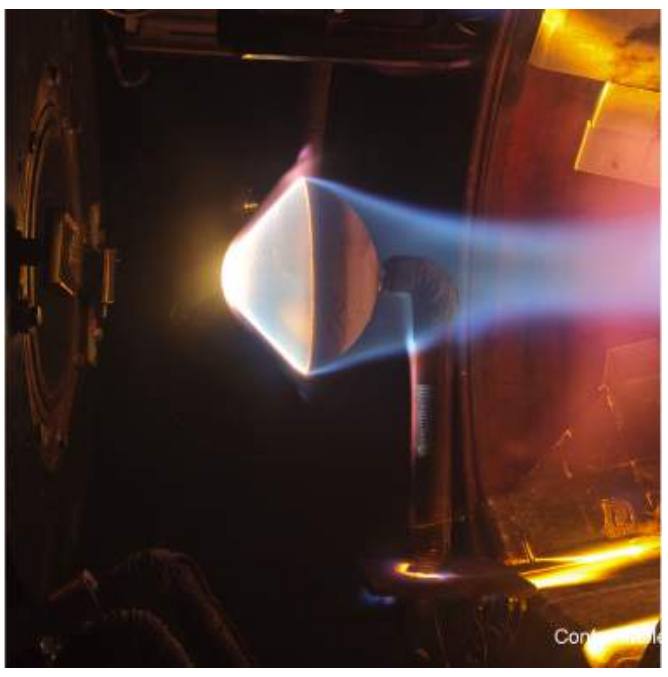

(b) Full scale SPRITE sample.

Figure 2.5.: The NASA-Ames Aerodynamic Heating Facility (Credit: NASA).

The AHF being an example of an arc-jet plasma wind tunnel, remarkable research on catalytic phenomena has been carried out in this type of facilities since the 1960's. The work of Winkler \& Griffin [56], or the one of Sheldahl et al. [57] are some examples. Particularly, the latter measured heat transfer rates on both silica and copper to study catalytic discontinuities. He showed that, when compared to a full body made of copper, the downstream heat transfer was significantly higher if a lower catalytic material such as silica was placed in the upstream nose of the test body. A more recent investigation was developed by Stewart et al. 58 for a catalytic coating applied on the Space Shuttle. In Europe, the Scirocco facility can be also used to visualize and quantify 
catalytic effects for the IXV. For instance, Trifoni [59] proposed a catalytic parameter based on heat flux measurements and numerical predictions on both non-catalytic and full-catalytic situations.

\subsubsection{The VKI-Plasmatron facility}

While arc-jets are extensively used to reproduce the thermal environment of a re-entry vehicle, small particles created by electrode erosion are present in the flow they provide. As a consequence, they lack the gas chemical purity produced by ICP wind tunnels. This fact makes the latter more suitable to measure the recombination coefficient of samples in flow conditions closely resembling the re-entry environment. The complete testing methodology has been developed by Barbante and Chazot [10] for the stagnation point.

The VKI-Plasmatron is a $1.2 \mathrm{MW}$ ICP facility able to use $2 \mathrm{kV}$ at $400 \mathrm{kHZ}$ to create the plasma, and expand it into a $2.5 \mathrm{~m}$ long and $1.4 \mathrm{~m}$ diameter test chamber through a $160 \mathrm{~mm}$ diameter torch. A scheme of the facility can be seen in Fig. 2.6. It can operate with different gases at both subsonic and supersonic regimes, with air, $\mathrm{N}_{2}$ and $\mathrm{CO}_{2}$ being the most commonly used. Test samples are normally located $445 \mathrm{~mm}$ from the torch exit. This is a standard configuration for most of the subsonic tests, adopted also in the present work. A heat exchanger is placed behind the chamber and is connected to the cooling system to ensure the appropriate gas temperature reduction before particles get into the vacuum pumps. Remark also that all metal surfaces exposed to plasma are cooled down with water to prevent them from melting.

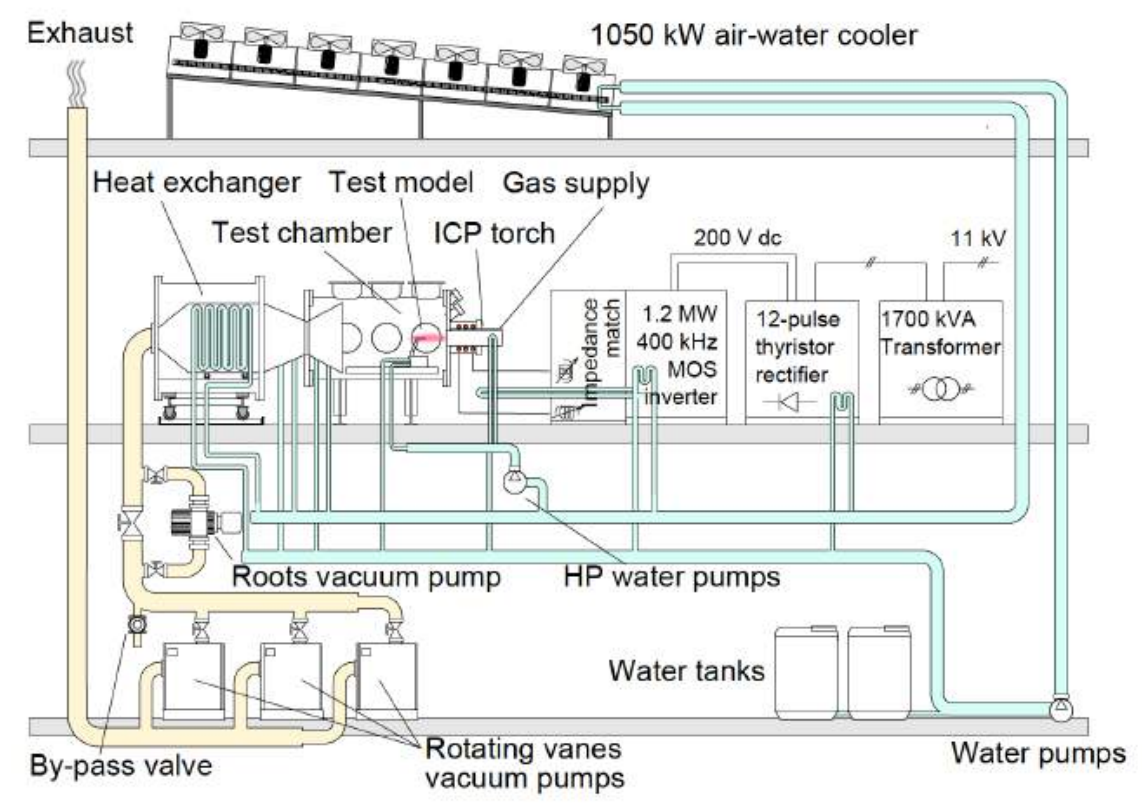

Figure 2.6.: The VKI-Plasmatron Facility.

Pressure ports allow different pressure measurements. Static pressure $P_{s}$ is measured at the top of the chamber with an absolute pressure transducer (Membranovac DM 12, Leybold Vacuum, OC Oerlikon Corporation AG, Switzerland) with $\pm 0.7 \mathrm{hPa}$ accuracy. Dynamic pressure $P_{d y n}$ is taken with a variable reluctance pressure transducer (DP-15, Valydine Engineering Corp, Northridge, CA USA) of $\pm 0.2 \%$ uncertainty. One of its ports is connected to the stagnant flow on a pitot probe, 
while the other has access to the static pressure line. It is assumed that uncertainties increase to $\pm 10 \%$ for $P_{s}$ due to the stability of the pumps regulating the vacuum conditions and to $\pm 20 \%$ for $P_{d y n}$ due to plasma jet fluctuations [11]. The gas mass flow rate is normally set at $16 \mathrm{~g} / \mathrm{s}$ and controlled with a rotameter. A picture of the Plasmatron test chamber is given in Fig. 2.7.

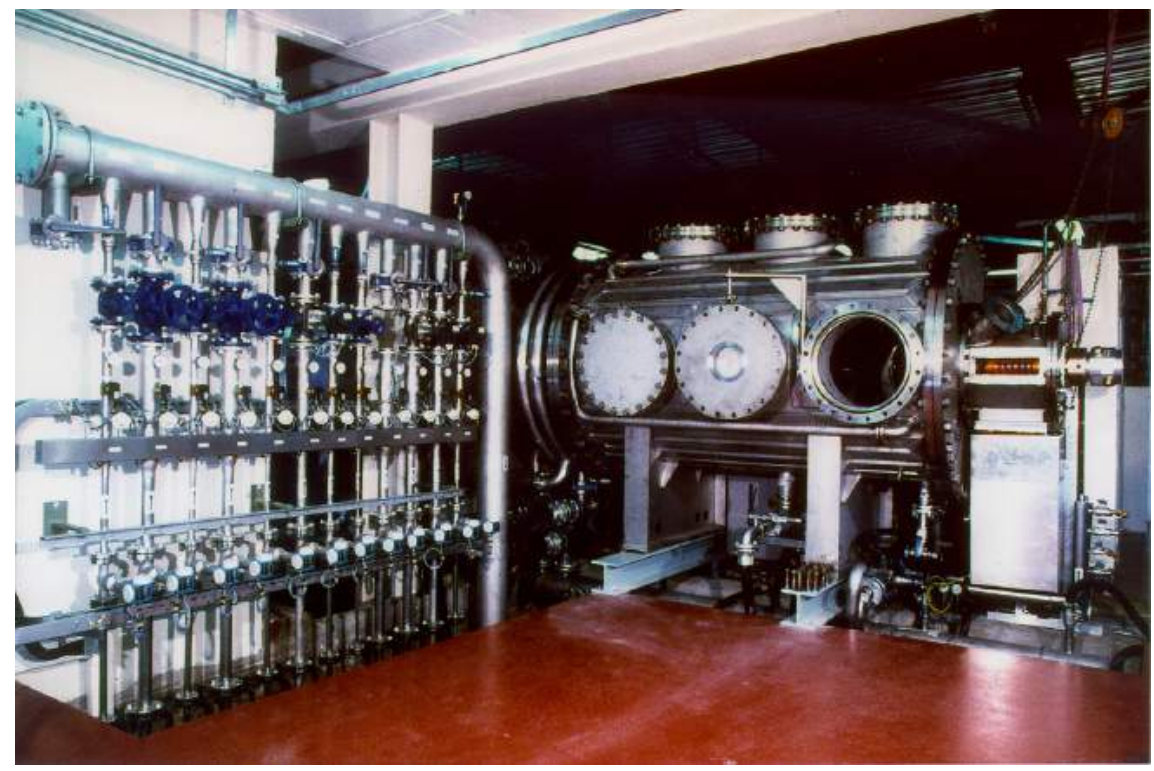

Figure 2.7.: The VKI-Plasmatron test chamber.

Although the flow configurations are quite different, a first attempt at comparing catalytic data between MESOX and the VKI-Plasmatron facilities was reported in 2009 by Chazot et al. [60]. While consistent results in terms of surface temperature and pressure were reported, recombination coefficients were not directly comparable because an energy accommodation coefficient of 1 is assumed at the VKI.

\subsection{On flight extrapolation for catalysis}

Although both diffusion tubes and plasma wind tunnels can be used to determine the recombination coefficient on TPS materials, the chemical regimes they provide can be very different. For instance, the former operate at very low pressures and can reach an almost (if not completely) frozen flow, and the latter reproduce a closer-to-flight situation, which takes place under higher pressure conditions and where flow chemistry is critical for heat transfer rates. This leads to different recombination coefficients determined in different types of facility, making the comparison of results between them quite difficult. As consequence, a problem arises when using experimental data to impose a catalytic recombination coefficient on CFD simulations trying to reproduce certain flight conditions: either one uses $\gamma$ defined under diffusion tubes and neglects the effect of pressure on the measurement, or one uses $\gamma$ determined in a plasma wind tunnel where, although the effect of pressure is relevant and surface reactions do not behave as first order, the recombination coefficient value is obtained under conditions closer to the re-entry environment.

Remark that the second option requires an extrapolation method to relate the appropriate testing conditions for catalytic determination on ground with the actual aerothermochemistry taking place 
in flight. This implies that certain combinations of altitude and velocity in the vehicle should be used to define the testing parameters in a plasma wind tunnel. Thus, the recombination coefficient determined under relevant-to-flight operating conditions can be confidently used as boundary condition in CFD solvers. Note that surface temperature, and sometimes also pressure, are the only parameters normally considered by literature to provide a recombination coefficient database, while the influence of probe geometry on the thermo-chemical environment under which $\gamma$ has been determined is normally disregarded.

An extrapolation methodology is proposed by Kolesnikov [7, 8] in the LHTS concept and has been adapted in the VKI by Barbante and Chazot [10], extended to ablative materials by Turchi et al. 9] and applied in a non-spherical re-entry configuration by Şakraker et al. [61]. The work is based on the non-equilibrium boundary layer equations analysis carried out in the late fifties. For instance, Fay \& Riddell [17] derived the following expression of heat flux assuming full-catalytic wall conditions:

$$
\dot{q}_{w}=0.763 \operatorname{Pr}^{-0.6}\left(\frac{\rho_{w} \mu_{w}}{\rho_{e} \mu_{e}}\right)^{0.1} \sqrt{\rho_{e} \mu_{e} \beta_{e}}\left(h_{e}-h_{w}\right)\left[1+\left(L e^{\alpha}-1\right) \frac{h_{D, e} c_{e}}{h_{e}}\right]
$$

where $\rho, \mu, h$ and $c$ are the flow density, viscosity, enthalpy and mass fraction, respectively. The subscripts $e$ and $w$ refer to the property evaluated at the boundary layer edge and at the wall of the body. Both $\operatorname{Pr}$ and Le are the Prandtl and Lewis numbers, and the exponent $\alpha$ is 0.63 for frozen boundary layers and 0.52 for equilibrium flows. The average dissociation enthalpy $h_{D, e}$ is defined as:

$$
h_{D, e}=\sum_{i}^{N_{s}} c_{i, e} h_{F, e}^{0}
$$

where $h_{F, e}^{0}$ is the dissociation energy of atomic products. Finally, the velocity gradient $\beta_{e}$ :

$$
\beta_{e}=\left.\frac{\partial u}{\partial x}\right|_{e}
$$

introduces the geometry of the body in the heat flux equation. On another publication, Goulard [15] performed a similar analysis, but under frozen boundary layers and with finite catalytic walls. Under these conditions, the heat flux was derived as:

$$
\dot{q}_{w}=0.664 \operatorname{Pr}^{-2 / 3} \sqrt{\rho_{e} \mu_{e} \beta_{e}} h_{e}\left[1+\left(L e^{2 / 3} \phi-1\right) \frac{h_{D, e} c_{e}}{h_{e}}\right]
$$

where surface catalysis is introduced through the parameter $\phi$ :

$$
\phi=\beta_{e}\left[1+\frac{0.47 S c^{-2 / 3} \sqrt{2 \rho_{e} \mu_{e} \beta_{e}}}{\rho_{w} k_{w}}\right]^{-1}
$$

Then, the LHTS concept states that the same boundary layer in flight can be obtained on ground if all the parameters in Eq. 2.12 and Eq. 2.15 are respected. Therefore, testing a TPS material with certain catalytic property $k_{w}$ and emissivity $\varepsilon$ leads to the same heat flux, and hence same surface temperature $T_{w}$, if the same $h_{e}, p_{e}$ and $\beta_{e}$ are imposed at the boundary layer edge assumed in LTE. Therefore, the flight and ground conditions are related through $h_{e}^{f}=h_{e}^{t}, p_{e}^{f}=p_{e}^{t}, \beta_{e}^{f}=\beta_{e}^{t}$, where 
superscripts $f$ and $t$ refer to flight and test, respectively. An illustration of the method is shown in Fig. 2.8 .

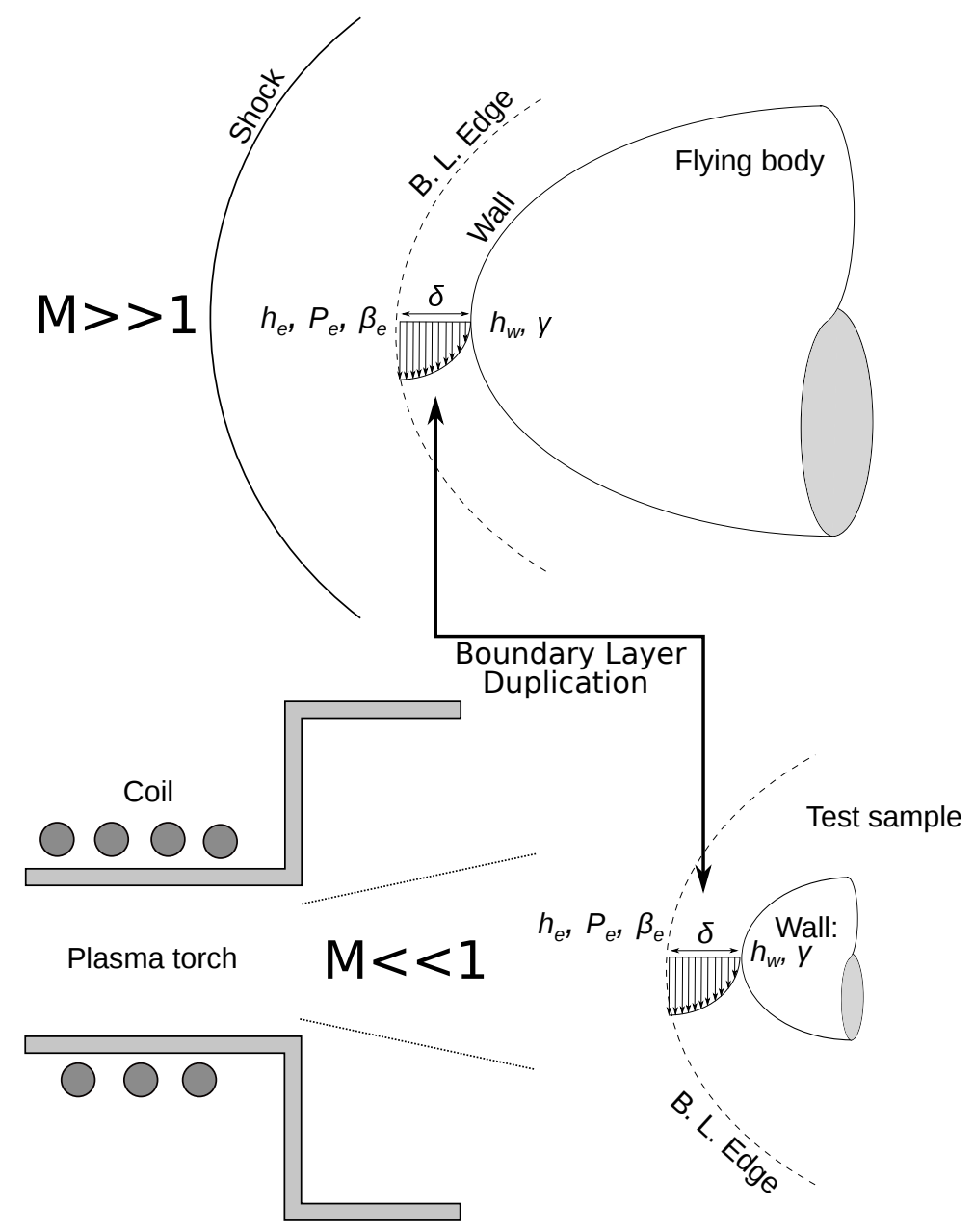

Figure 2.8.: Illustration of the LHTS concept on a Plasmatron facility.

Flight velocity and altitude can be used to define the thermodynamic parameters for a flight-toground extrapolation. The edge enthalpy is related to velocity with:

$$
h_{e}^{f}=h_{\infty}^{f}+\frac{1}{2} V_{\infty}^{2 f}
$$

and pressure is linked to altitude through $\rho_{\infty}$ with:

$$
p_{e}^{f}=p_{\infty}^{f}+\rho_{\infty}^{f} V_{\infty}^{2 f}
$$

Finally, the geometry of the flying body allows the estimation of the velocity gradient using modified Newtonian theory [1]. For a hemispherical flying body with nose radius $R_{e f f}, \beta_{e}$ reads:

$$
\beta_{e}^{f}=\frac{1}{R_{e f f}^{f}} \sqrt{2 \frac{p_{e}-p_{\infty}}{\rho_{e}}}
$$

Alternatively, the velocity gradient can also be estimated from thin shock layer theory [62] as: 


$$
\beta_{e}^{f}=\frac{1}{R_{e f f}^{f}} \sqrt{\frac{8}{3} \frac{p_{e}-p_{\infty}}{\rho_{e}}}
$$

Remark that both expression of the velocity gradient apply in hypersonics. However, many plasma wind tunnels like the VKI-Plasmatron operate in subsonic flow regimes. This means that an hemispherical effective radius for subsonic testing conditions can not be defined from Eq. 2.19 and Eq. 2.20. To overcome this issue, empirical relations that simplify the heat flux equation are used in both hypersonic and subsonic regimes. Zoby [63] derived the following simplified form for Eq. 2.12 with $L e=1$ for hypersonic conditions:

$$
\dot{q}_{w} \sqrt{\frac{R_{e f f, H}}{p_{0}}}=K_{i}\left(h_{e}-h_{w}\right)
$$

where $K_{i}$ is a constant depending on the gas mixture. Alternatively, a similar expression is defined for subsonic conditions [64] and reads:

$$
\dot{q}_{w} \frac{\sqrt{R_{e f f, S}}}{\sqrt[4]{p_{e} \Delta p}}=K_{i}\left(h_{e}-h_{w}\right)
$$

Therefore, combining Eq. 2.21 and Eq.2.22 and considering that $K_{i}$ is the same in both equations, the following relation between subsonic and hypersonic effective radius is obtained:

$$
R_{e f f, S}=R_{e f f, H} \frac{\sqrt{p_{e} \Delta p}}{p_{0}}
$$

where $\Delta p$ is the dynamic pressure. Remark that $p_{e} \Delta p$ refers to the ground condition and $p_{0}$ to the flight. 



\section{Chapter 3.}

\section{Enthalpy Characterization of the VKI-Plasmatron}

Although surface temperature is often taken as reference for TPS material qualification in plasma facilities, an accurate determination of the upstream flow properties is still necessary for development and application of extrapolation methodologies that link flight and ground conditions. Recall that it is important to test new TPS designs under thermal and chemical conditions that are representative to the flight, as discussed in Sec. 2.3, where the LHTS extrapolation methodology has been described. However, the free-stream characterization in plasma facilities and, more particularly, the enthalpy quantification, is still today an issue due to discrepancies found between different measurement techniques.

For instance, Park [65] presented four experimental methods applied on the Interaction Heating Facility (IHF) at NASA Ames Research Center: the heat balance method, the sonic throat method, the heat transfer method and the spectroscopic method. While the first two methods bring a mass averaged enthalpy quantification, the others provide an an enthalpy evaluation at the symmetry line of the plasma jet.

The heat balance method uses the ratio between the power applied to generate the plasma and the mass flow of gas to globally estimate the flow enthalpy. On the other hand, the sonic throat method assumes an inviscid equilibrium chocked flow at the nozzle's throat and makes use of:

$$
h_{0}=\left(0.293 \frac{A p_{0}}{\dot{m}}\right)^{2.519}
$$

to estimate the enthalpy. The heat transfer method takes the heat flux measured by a copper slug calorimeter installed a the center of a blunt body in a stagnant flow configuration, and plugs it into the formulation provided by Goulard [15] and Fay-Riddell [17] to calculate the symmetry line enthalpy. The relation of Fay-Riddell is given as:

$$
h_{0}=\frac{\dot{q}_{w}}{\dot{m}} \sqrt{\frac{R}{p_{0}}}\left(\frac{m_{A}}{K_{A}}+\frac{\dot{m}_{a}}{K_{a}}\right)
$$

where empirical constants $K_{A}=5.513 \times 10^{-4}$ and $K_{a}=3.905 \times 10^{-4}$ are taken from Zoby 63 for Argon and air respectively, and $R$ is the probe radius. The spectroscopic method consists on comparing some numerically generated spectra at different enthalpies (from $400 \mathrm{~nm}$ to $900 \mathrm{~nm}$ of wavelength in air), and the spectra measured with a spectrometer. The symmetry line enthalpy is determined by selecting the numerical spectra that best fits the experimental one. Park reported discrepancies of $\approx 40 \%$ between mass averaged and symmetry line methods. To mention that both the sonic throat and the heat transfer methods were already proposed by Pope [66] in 1968. He assumed, however, chemical equilibrium in the reservoir and frozen flow on the isentropic expansion 
along the nozzle for the former, and either full-catalytic or non-catalytic for the latter.

The methods presented by Park are specific to arc-jet facilities. At the VKI, the flow characterization techniques have been adapted to subsonic plasma jet and some other methods are available. One example is the enthalpy probe [67], which uses temperature measurements at the inlet and the outlet of a heat exchanger ( $T_{i n}$ and $T_{\text {out }}$, respectively) to determine the flow enthalpy. The design of the probe consists on a tubular heat exchanger of $6 \mathrm{~mm}$ of inner diameter and $14 \mathrm{~mm}$ of external diameter with water circulating inside. Such exchanger is inserted into a $50 \mathrm{~mm}$ diameter holder with a rounded edge of $11.75 \mathrm{~mm}$ radius, which is water cooled to prevent it from melting when exposed to the plasma jet. Both holder and heat exchanger are properly insulated one from each other, so energy from the gas is only transferred through the inner walls of the exchanger. A small pump that sucks the stagnant boundary layer into the exchanger is connected at the end of the probe, and the mass flow of gas $\dot{m}_{g}$ is measured with a rotameter. The temperature of the gas at the end of the exchanger $T_{2}$ is also measured by a type-E thermocouple. Enthalpy of the gas at the entrance of the probe $h_{1}$ can be determined with:

$$
h_{1}=c_{p} T_{2}+\frac{\dot{m}_{H_{2} O}}{\dot{m}_{g}}\left(T_{\text {out }}-T_{\text {in }}\right)
$$

which assumes the heat released by the gas to equal the energy received by the water circulating in the exchanger. The procedure to determine the plasma free-stream enthalpy consists on measuring $T_{\text {in }}, T_{\text {out }}$ and $T_{2}$ at different mass flows of gas passing through the heat exchanger. This provides a set of experimental data from which a regression curve of the type $h=A+B / \dot{m}_{g}$ can be defined. A plot illustrating the enthalpy probe configuration is shown in Fig. 3.1 .

However, such relation is not enough to determine the free-stream conditions because the optimal $\dot{m}_{g}$ is unknown. To define the appropriate suction rate one should consider that a $\dot{m}_{g}$ that is too high will modify the flow field around the probe and lead to an underestimation of the free-stream enthalpy. On the contrary, if $\dot{m}_{g}$ is too low, the measurement of enthalpy will be an overestimation of the free-stream condition. Therefore, the optimal suction rate is achieved when the flow velocity at the entrance of the heat exchanger corresponds to the value at the free-stream, which means that the stagnation point boundary layer is completely ingested by the enthalpy probe without altering the flow field. This condition requires the measurement of the dynamic pressure and is implemented as:

$$
\dot{m}_{g}=\rho V A=\sqrt{2 \rho P_{d y n}} A
$$

Then, solving the system of Eq. 3.3 3.4 iteratively, and assuming LTE at the boundary layer edge, the free-stream enthalpy can be determined.

Non-intrusive techniques based on Optical Emission Spectroscopy (OES) are also implemented in the Plasmatron. One of them is known as Real-Time Enthalpy Determination with Emission Spectroscopy (REDES) [67, 68]. The method is based on the work of Fletcher [69], which states that the molecular emission of $\mathrm{N}_{2}^{+}$first negative and $\mathrm{CN}$ violet is sensitive to air plasma temperature. These two transitions produce peaks around the $390 \mathrm{~nm}$ line of the spectrum. With the ratio between the peaks being proportional to temperature, one can numerically generate a calibration curve 


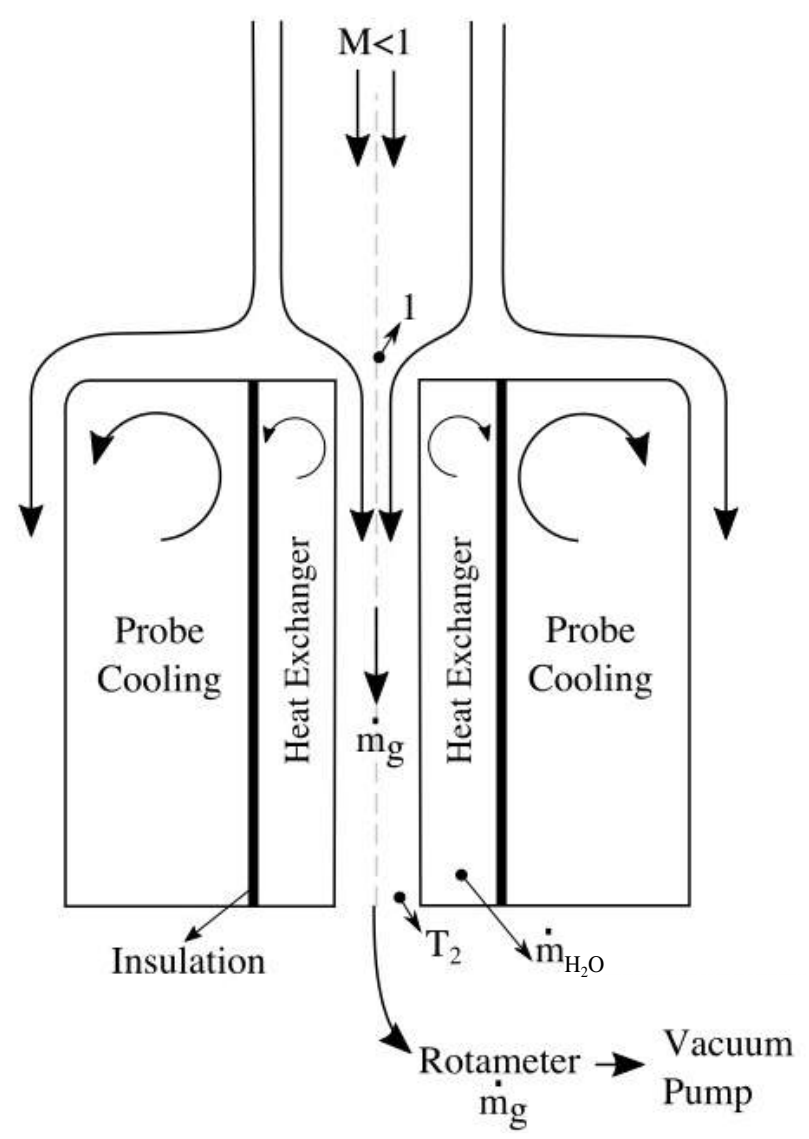

Figure 3.1.: Working principles of the enthalpy probe. Courtesy: Şakraker [67].

between this ratio and temperature using the software Specair [70]. By measuring the spectrum with an Ocean Optics HR4000 spectrometer, and introducing both the signal and the calibration curve into a LabView routine, one can determine the temperature of the plasma. Assuming the plasma under LTE, the temperature is easily converted into enthalpy using the Mutation ++ library [71]. A similar method was proposed by Cipullo [72, although enthalpy measurements were not in real time. Another OES method is proposed by Le Quang [73, 74], in which the radial temperature distribution of the plasma jet assumed in LTE can be obtained based on the oxygen triplet around the $777.7 \mathrm{~nm}$ wavelength with a Jobin-Yvon THR1000-MSL spectrometer and a Synapse CCD camera. Work on absorption/emission spectroscopy based on the LIF technique is also in progress at the VKI.

One additional enthalpy determination technique is revisited in this chapter. It is known as the Rebuilding method since it iterates with a non-equilibrium boundary layer solver that uses the measured quantities in the Plasmatron (heat flux, static and dynamic pressures) as input conditions to compute, as an inverser problem, the flow properties at the outer edge of the boundary layer. The determination of the recombination coefficient for a reference material and probe configuration is required as input condition to run the solver. The reference material being copper, an appropriate characterization of the catalytic activity on this material is necessary for the calibration of the Plasmatron in terms of measured heat flux and enthalpy. The rebuilding method has been used already in the past [11, but the reference catalytic conditions at the wall of the probe were taken 
from measurements in different types of facility. The fact that discrepancies are found among catalytic data obtained in different facilities encourages the in-house development of a reference catalytic model for copper.

\subsection{The enthalpy rebuilding method}

The logic of the rebuilding consists on measuring certain quantities of interest under certain test conditions in the Plasmatron, and using them as input conditions for two numerical solvers that reproduce the flow around the probe. The method provides a simulation of the chemically reacting boundary layer at the stagnation line, together with all the thermodynamic properties, from the outer edge to the wall. Although this method relies on both intrusive measurement techniques and several physico-chemical models to determine the outer edge enthalpy $H_{e}$ obtained at the Plasmatron, it becomes a powerful tool for investigations that require additional insight on specific physical phenomena.

The first software intervening in the rebuilding process is CoolFluid for ICP simulations. This solver helps to define the framework of the problem to be solved around the probe by simulating the interaction between the electromagnetic field around the coil and the gas passing through, with the aim of reproducing the whole Plasmatron chamber. ICP simulations are in LTE conditions, which is reasonable away from both the torch exit and the probe [73]. The CFD simulations carried out with this solver use a Finite Volume Method in a mesh with quadrilateral cells. All ICP computations performed for this dissertation consider an 11-species air mixture, the transport coefficients of which are computed with the VKI-developed Mutation ++ library [71]. In the simulations, all the walls are cooled down to $350 \mathrm{~K}$ and the annular injection of the gas is imposed at the inlet. Static pressure is imposed at the outlet of the fluid domain. An example of the temperature field computed in CoolFluid is shown in Fig. 3.2 .
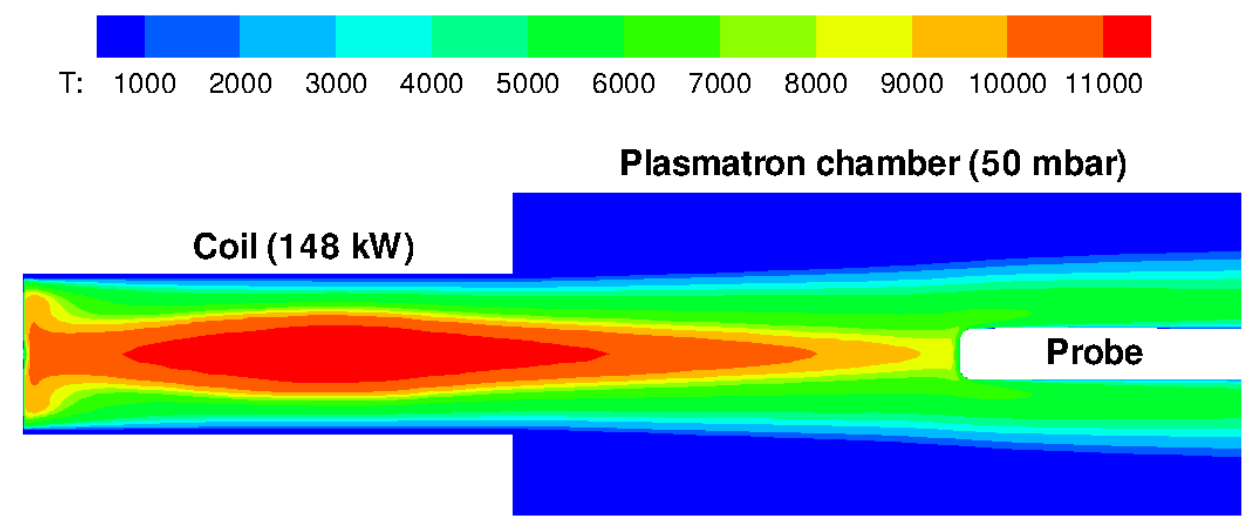

Figure 3.2.: Temperature field (in $\mathrm{K}$ ) provided by CoolFluid of a representative Plasmatron test simulation at $50 \mathrm{mbar}, 148 \mathrm{~kW}$ of power and $16 \mathrm{~g} / \mathrm{s}$ of mass flow.

The second numerical tool is the so-called Non-Equilibrium BOUndary LAyer (NEBOULA) solver developed by Barbante 22] in the VKI. It assumes thermal equilibrium and chemical non-equilibrium 
to compute the boundary layer around the probe in different flow configurations. Given the low Reynolds numbers in the Plasmatron (in the order of 100), the boundary layer solution should be obtained by solving the Navier-Stokes equations. However, they can be reduced to a set of ordinary differential equations like the second order boundary layer equations [75] or the Viscous Shock Layer (VSL) [76] equations for low Reynolds number, leading to a significant reduction of computational costs. If NEBOULA is used only for stagnation line, the second order boundary layer equations simplify to first order, and the boundary layer equations can be solved in this configuration to obtain an accurate solution.

For the purposes of this thesis, only the stagnation line option of NEBOULA will be considered. To solve the finite thickness boundary layer equations, the Lees-Dorodnitsyn transformation is applied for the independent variables $x$ and $y$ :

$$
\begin{gathered}
\xi(x)=\int_{0}^{x} \rho_{e} \mu_{e} u_{e} r^{2 \epsilon} d s \\
\hat{\eta}(x, y)=\mathcal{K} \frac{u_{e} r^{\epsilon}}{\sqrt{2 \xi}} \int_{0}^{y} \rho d t=\mathcal{K} \eta
\end{gathered}
$$

where:

$$
\mathcal{K}=\frac{1}{\delta} \frac{\sqrt{2 \xi}}{u_{e} r} \int_{0}^{\hat{\eta}_{\max }} \frac{1}{\rho} d \hat{\eta}
$$

The subscript $e$ refers to the properties evaluated at the outer edge, and $\epsilon$ is 0 for $2 \mathrm{D}$ and 1 for axisymmetric configurations. After this transformation, the system of boundary layer equations is written as:

$$
\begin{gathered}
\frac{\partial \tilde{V}}{\partial \hat{\eta}}+F=0 \\
\tilde{V} \frac{\partial y_{i}}{\partial \hat{\eta}}+\mathcal{K} \frac{\partial \tilde{\mathcal{J}}_{i}^{\hat{\eta}}}{\partial \hat{\eta}}=\dot{\mathcal{W}}_{i} \\
\tilde{V} \frac{\partial F}{\partial \hat{\eta}}=\frac{1}{2}\left(\frac{\rho_{e}}{\rho}-F^{2}\right)+\frac{1}{2} \frac{\rho_{e} v_{e}}{\rho\left(\frac{\partial u_{e}}{\partial x}\right)^{2}} \frac{\partial}{\partial y}\left(\frac{\partial u_{e}}{\partial x}\right)+\frac{\partial}{\partial \hat{\eta}}\left(\mathcal{K}^{2} l_{0} \frac{\partial F}{\partial \hat{\eta}}\right) \\
\tilde{V} \frac{\partial g}{\partial \hat{\eta}}=\frac{\partial}{\partial \hat{\eta}}\left(\mathcal{K}^{2} \frac{l_{0}}{\operatorname{Pr}} \frac{\partial g}{\partial \hat{\eta}}\right)-\frac{\partial}{\partial \hat{\eta}}\left(\mathcal{K}^{2} \frac{l_{0}}{P r} \sum_{i=1}^{N_{s}} \frac{\partial y_{i}}{\partial \hat{\eta}} \frac{h_{i}}{h_{e}}\right)-\frac{\partial}{\partial \hat{\eta}}\left(\mathcal{K} \sum_{i=1}^{N_{s}} \tilde{\mathcal{J}}_{i}^{\hat{\eta}} \frac{h_{i}}{h_{e}}\right)
\end{gathered}
$$

where the dependent variables are:

$$
\begin{gathered}
F=\frac{u}{u_{e}} \\
\tilde{V}=\mathcal{K} \frac{2 \xi}{\frac{\partial \xi}{\partial x}}\left(F \frac{\partial \eta}{\partial x}+\frac{\rho v r^{\epsilon}}{\sqrt{2 \xi}}\right) \\
g=\frac{h}{h_{e}}
\end{gathered}
$$


These equations are solved with a $4^{\text {th }}$ order Hermitian polynomial discretization in the direction perpendicular to the wall. The boundary conditions for this system are $F=1$ and $g=1$ at the outer edge, and $F=0$ and $g=h_{w} / h_{e}$ at the wall. The non-dimensional numbers appearing in this set of equations are the Chapman-Rubesin paramenter $l_{0}$, the mass production rate of species $i \dot{\mathcal{W}}_{i}^{1}$ and the transformed mass diffusion flux $\tilde{\mathcal{J}}_{i}^{\hat{\eta}}$, which are defined as:

$$
\begin{gathered}
l_{0}=\frac{\rho \mu}{\rho_{e} \mu_{e}} \\
\dot{\mathcal{W}}_{i}=\frac{\dot{\omega}_{i}}{2 \rho \frac{\partial u_{e}}{\partial x}} \\
\tilde{\mathcal{J}}_{i}^{\hat{\eta}}=\frac{J_{i}^{y}}{\sqrt{2 \rho_{e} \mu_{e} \frac{\partial u_{e}}{\partial x}}}
\end{gathered}
$$

An important fact to consider in reacting flows is how to describe diffusion phenomena. A simple diffusion mechanism is defined by Fick's law for binary mixtures, where $J_{i}^{y}=-D \frac{\partial \rho_{i}}{\partial y}$. However, this model does not respect the mass conservation in multi-component mixtures. A similar model is proposed by Ramshaw [5], in which the formulation of approximate diffusive fluxes is modified for an effective binary diffusion that respects the mass conservation equations. In NEBOULA, the Stefan-Maxwell equations are solved to model species diffusion [22]:

$$
\begin{gathered}
\vec{J}_{i}=-\rho \frac{M_{i}}{M} D_{i m} \overrightarrow{\tilde{d}}_{i}+y_{i} D_{i m} M \sum_{j=1}^{N_{s}} \frac{\vec{J}_{j}}{M_{j} \mathcal{D}_{i j}}+\frac{\rho}{p} \frac{M_{i}}{M} D_{i m}\left(\rho_{i} q_{i}-y_{i} \sum_{k=1}^{N_{s}} \rho_{k} q_{k}\right) \vec{E}_{a m b} \\
\sum_{i=1}^{N_{s}} q_{i} \vec{J}_{i}-\sum_{i=1}^{N_{s}} q_{i} y_{i} \sum_{j=1}^{N_{s}} \vec{J}_{j}=0
\end{gathered}
$$

This system of equations is solved with an iterative method to obtain $\vec{J}_{i}$ and the ambipolar electric field $\vec{E}_{a m b}$. This last parameter is introduced to ensure the so-called ambipolar constraint, that is the diffusion current is set to zero: $\sum_{i=1}^{N_{s}} q_{i} \vec{J}_{i}=0$. The species diffusion coefficients inside the mixture $D_{i m}$ are computed as:

$$
D_{i m}=\frac{1}{\sum_{j=1}^{N_{s}} \frac{x_{j}}{\mathcal{D}_{i j}}}
$$

and the binary diffusion coeffients $\mathcal{D}_{i j}$ are obtained from kinetic theory following:

$$
\mathcal{D}_{i j}=\frac{3}{16 n} \sqrt{\frac{2 \pi k_{B} T}{m_{i j}}} \frac{1}{\bar{\Omega}_{i j}^{11}}
$$

NEBOULA interacts with PEGASE library [77] to compute both the gas thermodynamic and transport properties. Different chemical models are available to compute the reaction rates. The condition of LTE is imposed at the boundary layer edge while $T_{w}$ and $\gamma$ are introduced as wall boundary conditions. Despite catalytic NO formation was detected by Copeland [20] in the side-

\footnotetext{
${ }^{1} \dot{\mathcal{W}}_{i}$ is the gas-phase Damköhler number of the system and it will be redefined in Ch. 4
} 
arm reactor described in Sec. 2.2.1.2, only two catalytic recombination reactions are considered relevant for an air mixture: $\mathrm{N}+\mathrm{N} \longrightarrow \mathrm{N}_{2}$ and $\mathrm{O}+\mathrm{O} \longrightarrow \mathrm{O}_{2}$. Catalysis is introduced through the diffusive flux evaluated at the wall, defined in Ref. [78] as:

$$
J_{i, w}^{y}=m_{i} \mathcal{M}_{i} \sum_{r=1}^{N_{r}} \nu_{r i} \gamma_{r}-\sum_{r=1}^{N_{r}} \sum_{j=1}^{N_{s}} \mu_{i j r} \gamma_{r} m_{j} \mathcal{M}_{j}
$$

This expression was proposed by Scott [79], in which $\nu_{r i}$ indicates the $i$ reactant colliding on the surface for the $r^{\text {th }}$ reaction, and matrices $\mu_{i j r}$ define the $j^{\text {th }}$ incident reactant producing the $i^{\text {th }}$ product through the $r^{\text {th }}$ reaction. The impinging particle flux $\mathcal{M}_{i}$ is implemented as:

$$
\mathcal{M}_{i}=n_{i} \sqrt{\frac{k_{B} T_{w}}{2 \pi m_{i}}}+\frac{J_{i, w}^{y}}{2 m_{i}}
$$

This equation is a particular case of its well-known definition $n_{i} \sqrt{k_{B} T_{w} / 2 \pi m_{i}}$ that allows the introduction of the first order perturbation term of Chapman-Enskog's expansion into Eq. 3.22 . With $\lambda_{w}$ being the thermal conductivity at the wall, one of the outputs of the boundary layer solver is a numerical heat flux $\overrightarrow{\dot{q}}_{\text {num }}$ at the wall, defined as:

$$
\overrightarrow{\dot{q}}_{n u m}=\lambda_{w}(\nabla T)_{w}+\sum_{i=1}^{N_{s}} \vec{J}_{i, w} h_{i, w}
$$

NEBOULA is plugged into a routine named CERBERE2 in order to be used as a tool for Plasmatron data post-processing. The input variables of CERBERE being a combination of measured quantities in the Plasmatron and some hydrodynamic outputs from ICP simulations, it iterates with NEBOULA in order to rebuild the stagnation line boundary layer in front of the probe and to provide a value of the recombination coefficient on TPS material samples.

In the framework of the rebuilding method, the coupling between CERBERE and NEBOULA is commonly known as CERBOULA, and it can provide either an outer edge enthalpy $H_{e}$ to characterize the plasma if wall catalysis is known or a recombination coefficient $\gamma$ on a reference material if $H_{e}$ is given, but never both variables at the same time. The typical relation between unknowns $H_{e}$ and $\gamma$ is shown in Fig. 3.3 for a heat flux of $1100 \mathrm{~kW} / \mathrm{m}^{2}$ and a surface temperature of $350 \mathrm{~K}$.

The operating conditions of the Plasmatron are defined by gas mass flow $\dot{m}$, static pressure $P_{s}$ and power $P W$. Note that the latter is the power at the coil times an efficiency $\left(\eta_{\text {plasma }}\right)$ assumed to be $50 \%$ [80]. These quantities, together with the probe configuration (or the radius $R_{b}$ ), are used as input for the ICP simulations, the output of which is used as input for CERBOULA in the form of 5 Non-Dimensional Parameters $\Pi_{i}(\mathrm{NDP})$. They are defined as follow:

- non-dimensional thickness: $\Pi_{1}=\frac{\delta}{R_{b}}$

- non-dimensional velocity gradient: $\Pi_{2}=\left(\frac{\partial u}{\partial x}\right)_{e} \frac{R_{b}}{v_{s}}$

- non-dimensional velocity gradient derivative: $\Pi_{3}=\frac{\partial}{\partial y}\left(\frac{\partial u}{\partial x}\right)_{e} \frac{R_{b}^{2}}{v_{s}}$

- non-dimensional velocity: $\Pi_{4}=\frac{v_{e}}{v_{s}}$

\footnotetext{
${ }^{2}$ CERBERE: Catalycity and Enthalpy ReBuilding on a REference probe
} 


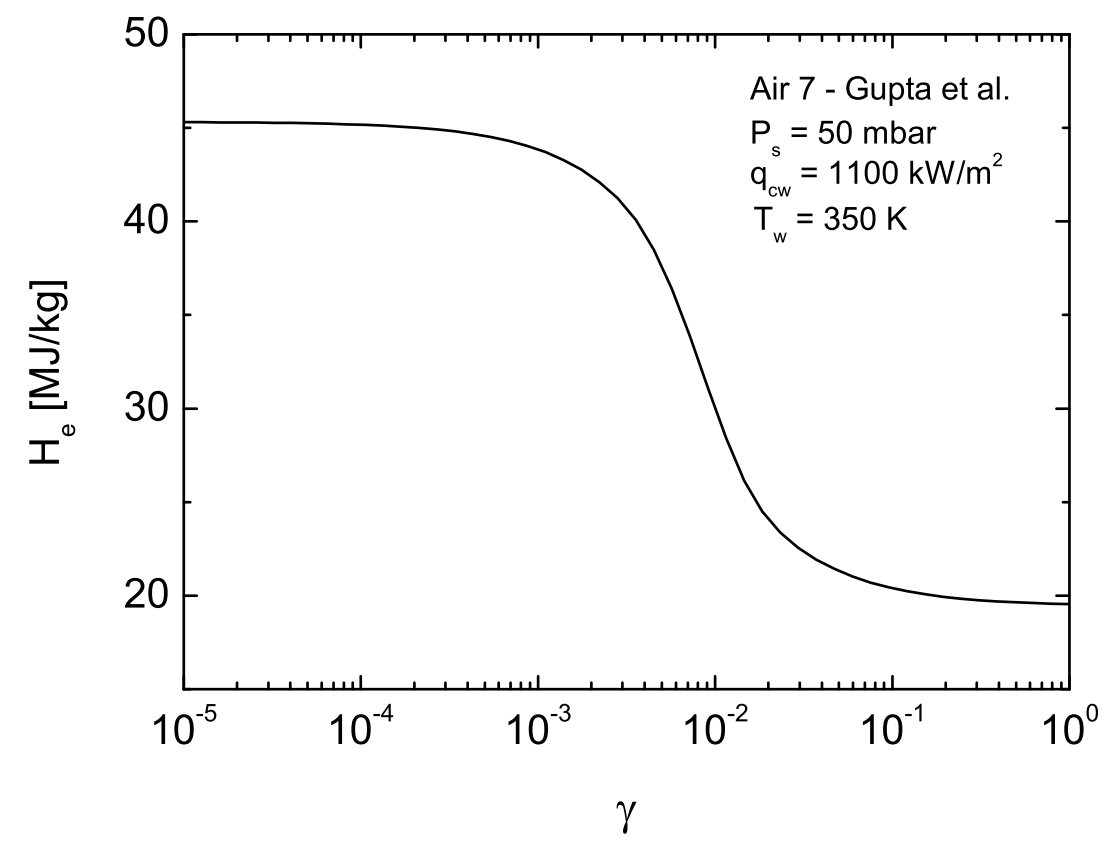

Figure 3.3.: Relation between catalysis and boundary layer outer edge enthalpy in a Plasmatron test.

- modified non-dimensional velocity: $\Pi_{5}=\frac{v_{e}}{v_{e, d}}$

Note that $v_{s}$ is the velocity at the exit of the torch and $v_{e, d}$ the velocity at the same location as the boundary layer edge, but for an ICP solution without probe.

Other variables that are introduced for enthalpy rebuilding are a dynamic pressure measurement $P_{d y n}$ and a cold wall heat flux measurement $\dot{q}_{c w}$ taken on a copper calorimeter. The last inputs required by CERBOULA concern the wall conditions of the calorimeter in terms of temperature $T_{w}$ and a reference recombination coefficient on cooled copper $\gamma_{r e f}$. The former is assumed to be constant at $350 \mathrm{~K}$ [81, and the latter is to be defined in Sec. 3.2. The outer edge enthalpy being an input for NEBOULA, the numerical $\dot{q}_{n u m}$ can be computed. Then, CERBERE iterates with NEBOULA using the measured $\dot{q}_{c w}$ to establish the convergence criteria, giving $H_{e}$ as output of the process. A scheme of the classical enthalpy rebuilding methodology is shown in Fig. 3.4.

The heat flux measurement for $H_{e}$ rebuilding is taken with the $14 \mathrm{~mm}$ in diameter flat faced calorimeter shown in Fig. 3.5. It is introduced at the center of the $50 \mathrm{~mm}$ diameter holder with $11.75 \mathrm{~mm}$ radius on the edge to form the ESA-Standard probe shown in Fig. 3.6. Both calorimeter and holder are made of copper and they are water-cooled by independent circuits to prevent them from melting. The insulation between calorimeter and holder is achieved with a Teflon ring between the two elements. Two type-E thermocouples are installed at both inlet and outlet of the calorimeter. The temperature difference $T_{\text {out }}-T_{\text {in }}$, the water mass flow rate $\dot{m}_{\mathrm{H}_{2} \mathrm{O}}$ and the calorimeter area $A$ allow the real time heat flux measurement in steady-state with:

$$
\dot{q}_{c w}=\frac{\dot{m}_{H 2 O} c_{p}}{A}\left(T_{o u t}-T_{i n}\right)
$$




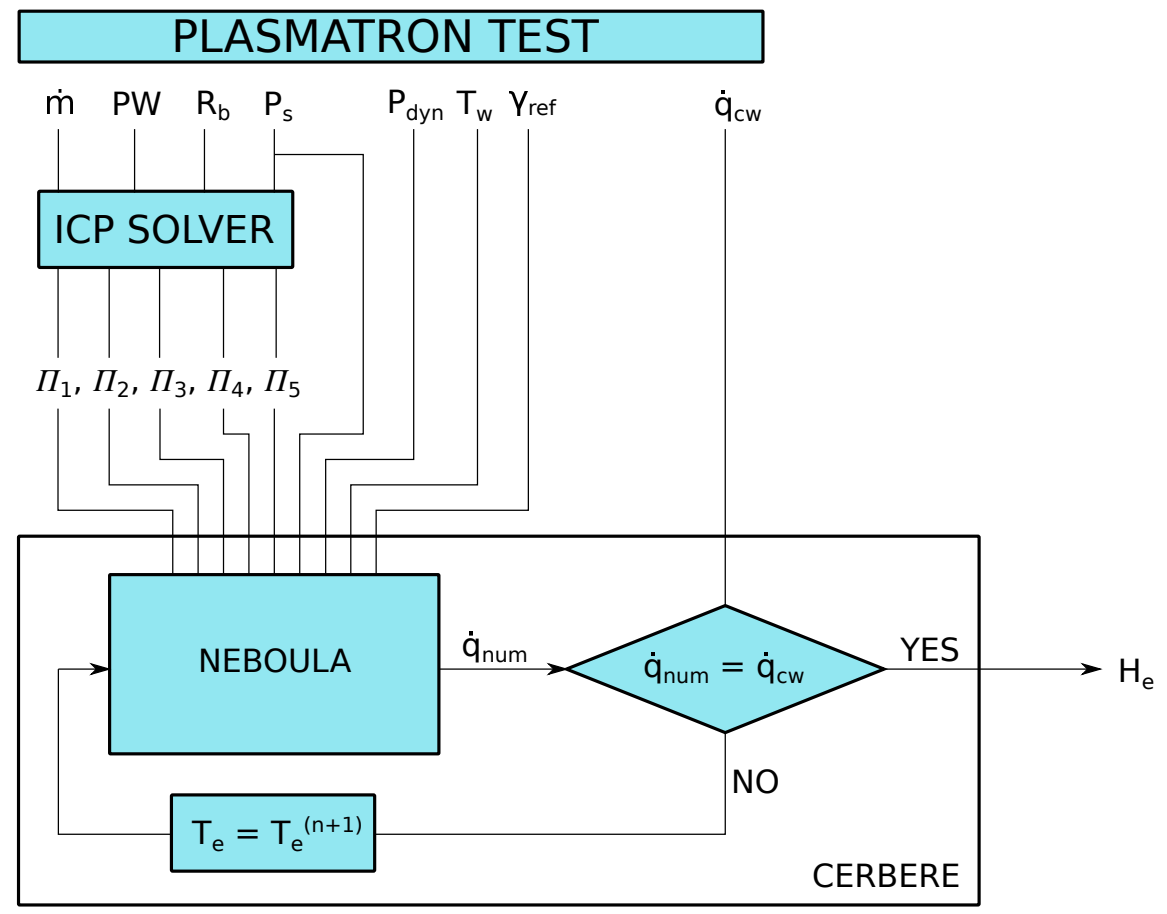

Figure 3.4.: Diagram of the $H_{e}$ rebuilding method.

An uncertainty of $\pm 10 \%$ is expected considering the values in Table 3.1 and the following expression [11:

$$
\frac{\delta \dot{q}_{c w}}{\dot{q}_{c w}}=\sqrt{\left(\frac{\delta \dot{m}_{H 2 O}}{\dot{m}_{H 2 O}}\right)^{2}+\left(\frac{\delta c_{p}}{c_{p}}\right)^{2}+\left(\frac{\delta\left(T_{o u t}-T_{\text {in }}\right)}{T_{\text {out }}-T_{\text {in }}}\right)^{2}+\left(\frac{\delta A}{A}\right)^{2}}
$$

Table 3.1.: Uncertainties of measured quantities

\begin{tabular}{cr}
\hline \hline Measured Quantity & Uncertainty \\
\hline$\dot{m}$ & $\pm 0.5 \mathrm{mg} / \mathrm{s}$ \\
$\Delta T$ & $\pm 0.8 \mathrm{~K}$ \\
$c_{p}$ & $\pm 0.1 \mathrm{Jkg}^{-1} \mathrm{~K}^{-1}$ \\
$A$ & $\pm 4.4 \mathrm{~mm}^{2}$ \\
\hline \hline
\end{tabular}

The main problem to apply the enthalpy rebuilding method is the lack of knowledge on copper catalysis. Values of the recombination coefficient on copper and cupric oxide are provided by several authors [29, 31, 50, 82, 88]. Panerai [11] collected data of recombination coefficients on copper from literature [15, 49, 89, 92] and proposed the following model for $\gamma_{r e f}$ :

$$
\gamma_{r e f}\left(P_{s}\right)= \begin{cases}0.1 & \text { if } 1200 \geq P_{s} \leq 5000 \mathrm{~Pa} \\ 0.01 & \text { if } 5000<P_{s} \leq 10000 \mathrm{~Pa} \\ 0.005 & \text { if } P_{s}>10000 \mathrm{~Pa}\end{cases}
$$

However, Kovalev [93] mentioned discrepancies in catalytic data for highly-catalytic materials 


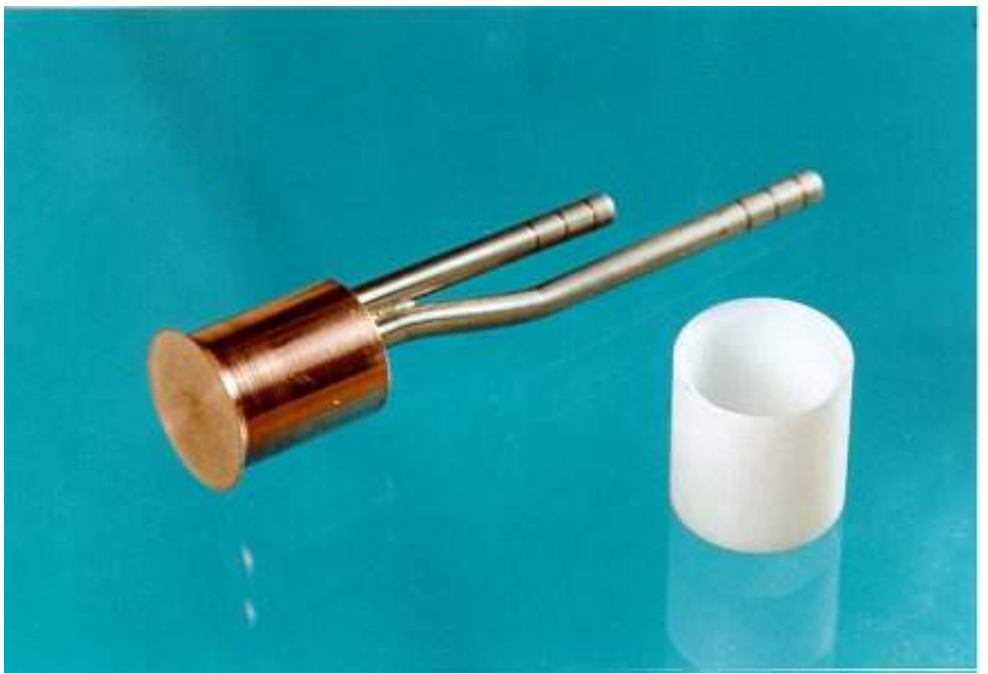

Figure 3.5.: Copper calorimeter and Teflon ring.

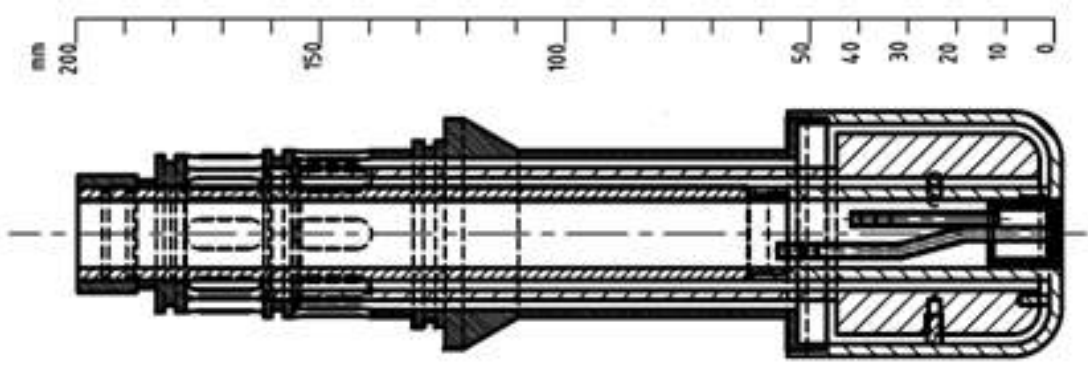

Figure 3.6.: ESA-Standard probe with calorimeter at the center.

coming from different plasma facilities. The testing campaign presented below consolidates a methodology for an in-house estimation of copper catalysis on the ESA-Standard probe. The advantage of the method is that it is directly applied to the calorimeter intended to be used for enthalpy determination at any test condition of the Plasmatron facility.

\subsection{The Mini-Max methodology}

The so-called Mini-Max method can provide a reference catalytic model for the copper calorimeter shown in Fig. 3.5 using the ESA-Standard probe, so that the outer edge enthalpy $H_{e}$ conditions can be determined with the rebuilding method for a wide range of Plasmatron testing conditions. The reference recombination coefficient is named $\gamma_{r e f}$ and its modelling is used as a calibration parameter to relate the numerically rebuilt $H_{e}$ with the heat flux measurements on the copper calorimeter. Experiments presented in the following section provide a quantification of $\gamma_{r e f}$ so that a reference catalytic model can be developed in the enthalpy rebuilding framework.

The logic behind the Mini-Max method is simple. Three heat flux measurements are taken on three different calorimeters under the same plasma conditions. Calorimeters being of different materials, those dedicated to the Mini-Max in air plasma are silver, copper and Quartz. Differences in $\dot{q}_{c w}^{(i)}$ are consequence of different catalytic activities on the surface of each calorimeter. The three 
heat flux measurements provide, when introduced in CERBOULA, three $H_{e^{-}} \gamma$ relations like the shown in Fig. 3.3 .

Considering both silver and Quartz as the high catalytic $\left(\gamma_{A g} \rightarrow 1\right)$ and low catalytic $\left(\gamma_{Q z} \rightarrow 0\right)$ standards, respectively, it is possible to define an enthalpy range inside which the testing conditions should be found. Thus, a minimum enthalpy $H_{e, \min }$ is given by the silver calorimeter and a maximum enthalpy $H_{e, \max }$ by the Quartz calorimeter. An example is shown in Fig. 3.7. The intersection of this interval with the $H_{e^{-}} \gamma$ relation obtained on copper defines a catalytic range for the testing conditions at hand, with a maximum recombination coefficient $\gamma_{\max }$ given by $H_{e, \min }$ and a minimum $\gamma_{\min }$ provided by $H_{e, \max }$. The average value between both recombination coefficients will be considered as $\gamma_{r e f}$. Remark that Krassilchikoff similarly applied the Mini-Max with molybdenum instead of quartz [91].

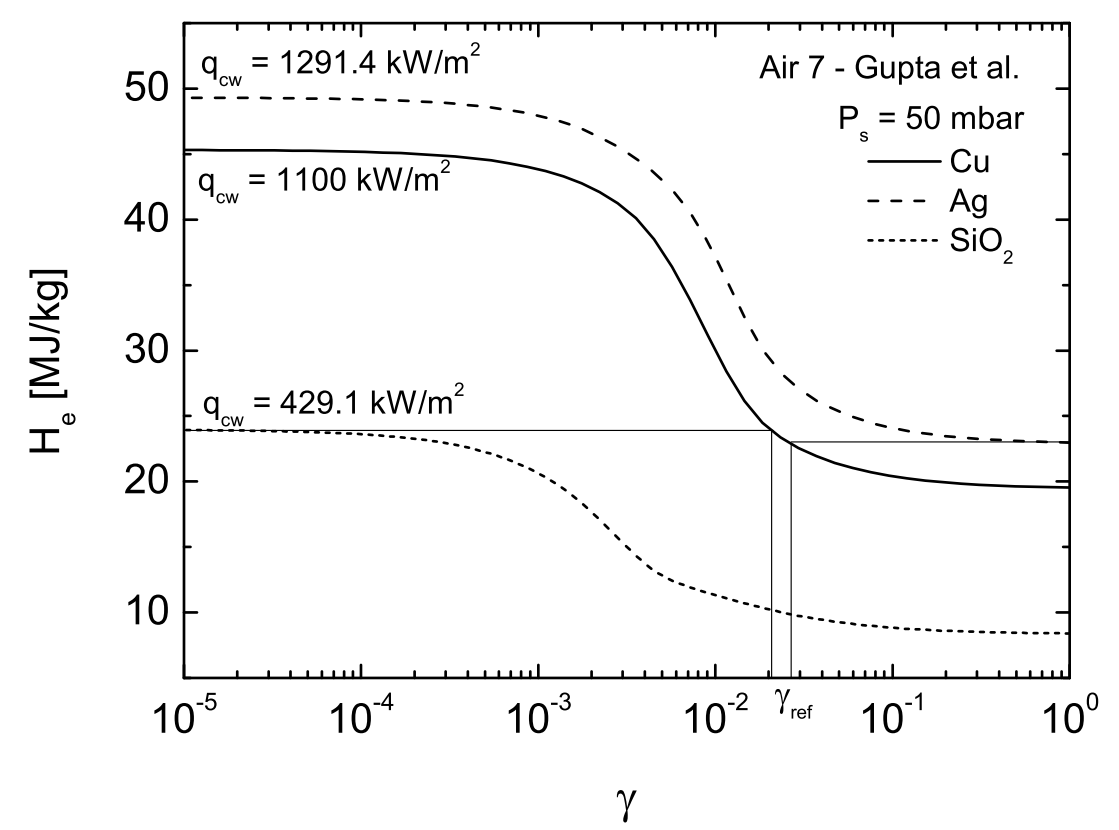

Figure 3.7.: Illustration of Mini-Max method application.

It is worth reminding the assumptions made in CERBOULA to anticipate possible limitations of the rebuilding method:

- In terms of catalysis, the hypothesis $\gamma_{N}=\gamma_{O}$ and $\gamma_{N O}=0$ is made for convenience. One way to verify this assumption is testing with pure $\mathrm{N}_{2}$ and pure $\mathrm{O}_{2}$ separately to define independently $\gamma_{N}$ and $\gamma_{O}$, respectively. However, testing with nitrogen on one hand would show that copper is better catalyst than silver, making the copper catalysis determination of $\gamma_{N}$ impossible through the Mini-Max. On the other hand, testing with pure oxygen presents some safety issues as it can endanger the facility because of the hot gas reaching the vacuum pumps. Therefore, the $\gamma_{\text {ref }}$ provided in this testing campaign should be seen as a parameter that reasonably closes the boundary condition of the species conservation equation, rather than a true chemical reaction rate constant. Note that an Uncertainty Quantification (UQ) analysis 
based on Bayesian inference [94 could provide more information about the validity of the $\gamma_{N}=\gamma_{O}$ hypothesis using data provided by this dissertation.

- The surface temperature on the calorimeter is normally assumed constant for all power ranges: $350 \mathrm{~K}$ on both copper and silver, and $750 \mathrm{~K}$ on Quartz. Indeed, with water-cooled calorimeters, only small variations of surface temperature with Plasmatron power are expected, and they are not supposed to have a relevant impact on $H_{e}$.

\subsection{The Mini-Max testing application}

A dedicated testing campaign for Mini-Max methodology application is carried out following the scheme shown in Fig. 3.8. The three calorimeters of Fig. 3.9 are inserted in the ESA-Standard probe, located at $445 \mathrm{~mm}$ from the torch exit and aligned with the center of the plasma jet. Note that both copper and silver calorimeter surfaces are almost black (Fig. 3.9). This is due to copper oxidation into $\mathrm{Cu}_{2} \mathrm{O}$ and silver nitridation into $\mathrm{Ag}_{3} \mathrm{~N}$ caused by previous air and nitrogen plasma exposures. As both cupric oxide and silver nitride processes reach a steady-state, the calorimeters are intentionally left unpolished for Mini-Max application because oxidation and nitridation processes in plasma are very fast, and polishing the calorimeters at each test condition could turn impractical for long testing campaigns. Therefore, one should note that the recombination coefficient determined with the Mini-Max is not on pure copper, but on $\mathrm{Cu}_{2} \mathrm{O}$. This is not supposed to become an inconvenient for the rebuilding given the fact that only catalysis on a reference material is really needed.

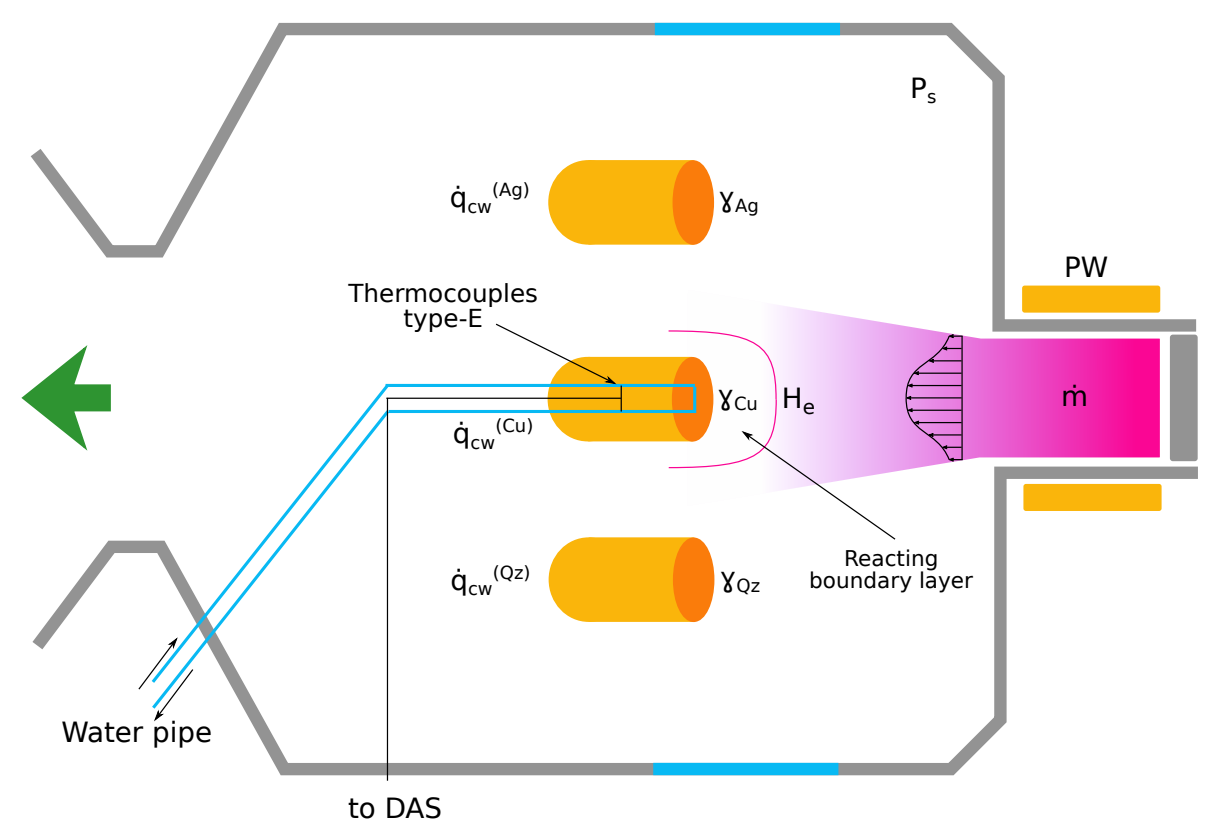

Figure 3.8.: Plasmatron test configuration for $\gamma_{r e f}$ determination with the Mini-Max.

Four static pressure $P_{s}$ conditions are considered for this testing campaign: 15, 50, 100 and 200 mbar, identified as test conditions a, b, c and d, respectively. All measurements are taken under air 


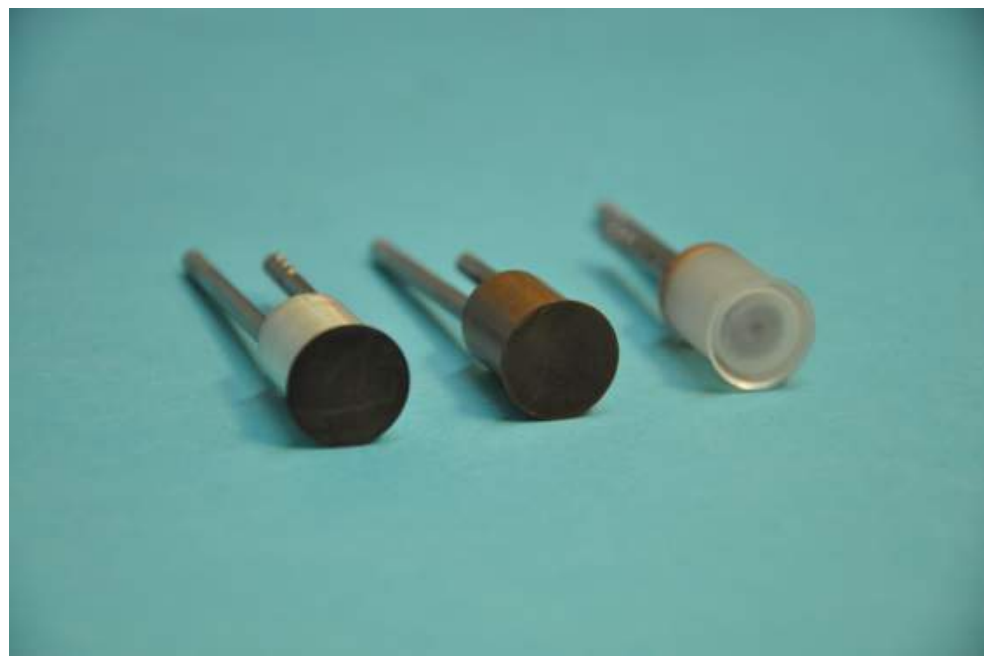

Figure 3.9.: Calorimeters for Mini-Max methodology application: left - Ag, middle - Cu, right $\mathrm{SiO}_{2}$-Quartz.

plasma at $16 \mathrm{~g} / \mathrm{s}$. Plasmatron power is adapted in order to target certain $\dot{q}_{c w}^{(r e f)}$ reading on the copper calorimeter. Nine heat flux values are sampled for each $P_{s}$. This allows to obtain a distribution of $\gamma$ throughout different plasma power conditions. Dynamic pressure $P_{d y n}$ has been measured under the same configuration prior to Mini-Max application because only three samples can be introduced in the Plasmatron test chamber during the same run. Dynamic pressure measurements are provided in Table A.1.

To proceed, the Plasmatron is switched on and the air mass flow is set with a rotameter. Then, the vacuum pumps are regulated until the target static pressure is reached inside the chamber. After that, the probe with the copper calorimeter is injected into the plasma and power $P W$ is regulated according to the target heat flux being measured, displayed and recorded in real-time at $1 \mathrm{~Hz}$. Once the calorimeter reaches a steady-state signal under the imposed conditions, the probe is removed from the plasma jet and that holding the silver calorimeter is introduced. The heat flux is measured and the injection/ejection process is repeated for Quartz. The testing conditions and measurements are listed in Table 3.2 , where the first three columns are the test denomination, the target heat flux and the electric power respectively, followed by the heat flux measurements on the three calorimeters.

The fact that $\dot{q}_{c w}^{(A g)}>\dot{q}_{c w}^{(C u)}>\dot{q}_{c w}^{(Q z)}$ confirms that $\gamma_{A g}>\gamma_{C u}>\gamma_{Q z}$ in air plasma. Note that tests 1a, $2 \mathrm{a}$ and $3 \mathrm{a}$ are the only exceptions to this rule because heat flux on copper is higher than on silver. Remark that, across these three conditions, the maximum heat flux difference between silver and copper is $12 \%$ (case $2 \mathrm{a}$ ), which falls close to the $10 \%$ of uncertainty in heat flux, as given by Eq. 3.26. Therefore there is no significant difference between the heat fluxes on the silver and copper calorimeters. This is reasonable due to the small nitrogen dissociation level at low pressures and powers. Under such conditions, nitrogen recombination mechanisms play a minimal role in the heat flux measurements, which become insensitive to catalytic effects. Note that measurements on Quartz are lower because the surface temperature increases to $750 \mathrm{~K}$, which is higher than the 350 $\mathrm{K}$ reached on both silver and copper. 
Table 3.2.: Measurements of Mini-Max testing campaign at: (a) 15 mbar, (b) 50 mbar, (c) 100 mbar, (d) 200 mbar

\begin{tabular}{|c|c|c|c|c|c|}
\hline Test & 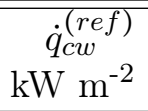 & $\begin{array}{r}P W \\
\mathrm{~kW}\end{array}$ & 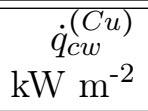 & 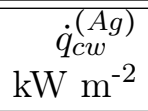 & 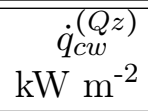 \\
\hline $1 \mathrm{a}$ & 300 & 106 & 314.5 & 298.5 & 136.8 \\
\hline $2 \mathrm{a}$ & 500 & 145 & 521.5 & 462.9 & 157.1 \\
\hline $3 a$ & 700 & 169 & 709.3 & 692.2 & 233.8 \\
\hline $4 a$ & 900 & 204 & 907.6 & 959.1 & 347.9 \\
\hline $5 a$ & 1100 & 254 & 1098.1 & 1229.4 & 451.9 \\
\hline $6 \mathrm{a}$ & 1300 & 275 & 1329.4 & 1498.4 & 626.5 \\
\hline $7 \mathrm{a}$ & 1500 & 304 & 1529.0 & 1799.5 & 738.3 \\
\hline $8 a$ & 1700 & 330 & 1708.8 & 2030.8 & 819.2 \\
\hline $9 a$ & 2000 & 360 & 1997.2 & 2345.3 & 902.0 \\
\hline $1 \mathrm{~b}$ & 300 & 128 & 306.1 & 349.5 & 144.1 \\
\hline $2 b$ & 500 & 153 & 511.3 & 575.2 & 190.4 \\
\hline $3 b$ & 700 & 190 & 716.5 & 749.1 & 223.1 \\
\hline $4 \mathrm{~b}$ & 900 & 228 & 902.8 & 1074.6 & 278.3 \\
\hline $5 b$ & 1100 & 256 & 1088.7 & 1315.4 & 380.1 \\
\hline $6 \mathrm{~b}$ & 1300 & 282 & 1295.8 & 1553.7 & 486.1 \\
\hline $7 \mathrm{~b}$ & 1500 & 291 & 1544.8 & 1840.2 & 684.7 \\
\hline $8 \mathrm{~b}$ & 1700 & 349 & 1700.7 & 2000.9 & 754.1 \\
\hline $9 b$ & 2000 & 426 & 2003.6 & 2369.7 & 795.1 \\
\hline $1 \mathrm{c}$ & 300 & 128 & 301.8 & 420.7 & 289.4 \\
\hline $2 c$ & 500 & 157 & 504.8 & 573.9 & 404.5 \\
\hline $3 c$ & 700 & 175 & 706.4 & 789.0 & 498.5 \\
\hline $4 c$ & 900 & 195 & 905.4 & 997.8 & 537.6 \\
\hline $5 c$ & 1100 & 229 & 1102.6 & 1223.5 & 560.9 \\
\hline $6 c$ & 1300 & 259 & 1322.5 & 1430.7 & 660.2 \\
\hline $7 \mathrm{c}$ & 1500 & 268 & 1521.2 & 1652.6 & 739.6 \\
\hline $8 c$ & 1700 & 294 & 1701.3 & 1905.7 & 815.9 \\
\hline $9 \mathrm{c}$ & 2000 & 343 & 2024.7 & 2271.0 & 892.7 \\
\hline $1 d$ & 300 & 137 & 303.3 & 331.0 & 217.3 \\
\hline $2 \mathrm{~d}$ & 500 & 168 & 506.8 & 550.6 & 327.8 \\
\hline $3 \mathrm{~d}$ & 700 & 182 & 716.2 & 783.0 & 429.7 \\
\hline $4 d$ & 900 & 197 & 909.7 & 972.9 & 544.8 \\
\hline $5 \mathrm{~d}$ & 1100 & 210 & 1115.2 & 1176.5 & 648.4 \\
\hline $6 \mathrm{~d}$ & 1300 & 225 & 1317.0 & 1391.4 & 748.4 \\
\hline $7 \mathrm{~d}$ & 1500 & 246 & 1513.4 & 1625.2 & 828.5 \\
\hline $8 \mathrm{~d}$ & 1700 & 262 & 1710.6 & 1825.7 & 880.6 \\
\hline $9 \mathrm{~d}$ & 2000 & 291 & 2033.9 & 2162.9 & 956.5 \\
\hline
\end{tabular}




\subsection{Reference recombination coefficient determination}

The behavior of the heat flux measurements is linear with the reference heat flux. An example is shown in Fig. 3.10 from condition $1 \mathrm{~b}$ to $9 \mathrm{~b}$ (50 mbar). Heat flux measurements are interpolated with a linear fit to match the target conditions on copper. The resulting parameters for the interpolation are provided in Table 3.3 The same procedure is applied to power and to dynamic pressure measurements (see Table A.2. By following this step, it is possible to relate $\dot{q}_{c w}^{(i)}, P W$ and $P_{d y n}$ measurements to the same reference testing conditions and prepare the data for post-processing.

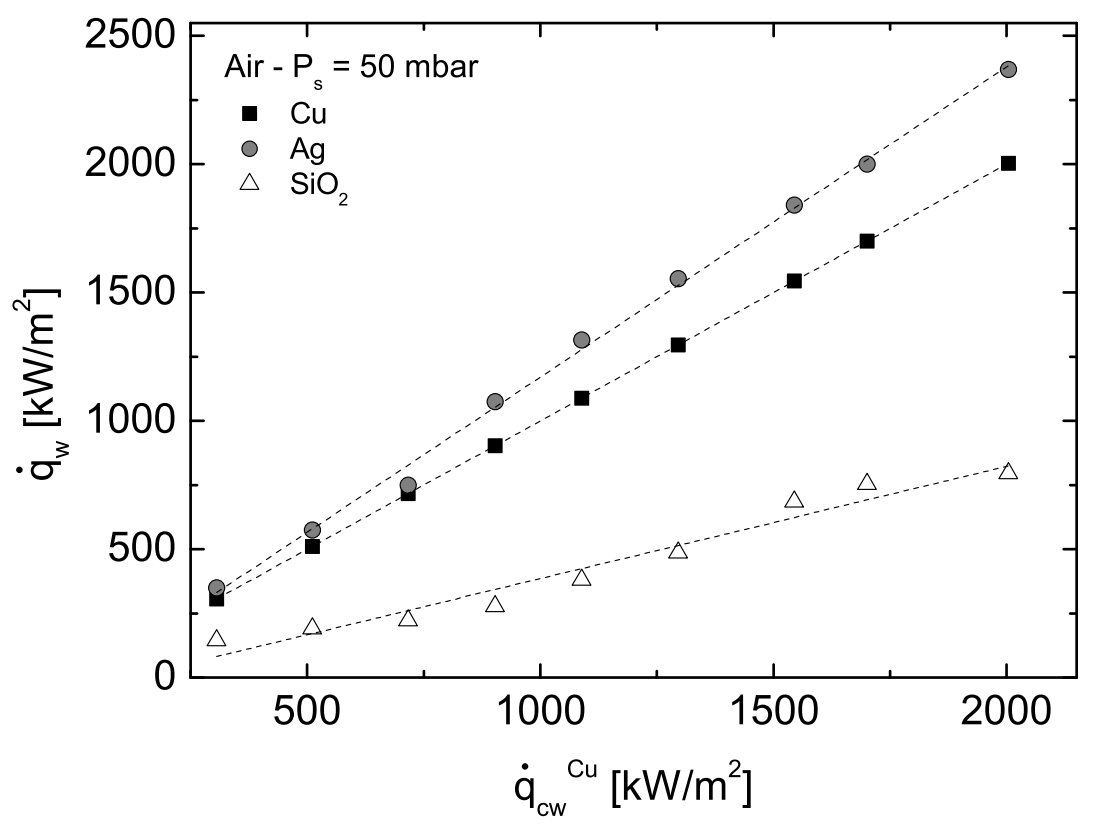

Figure 3.10.: Heat flux measurements for Mini-Max application at 50 mbar. Regressions are used for data post-processing in CERBERE.

Table 3.3.: Linear interpolation coefficients for the heat fluxes measured during the Mini-Max method application $\left(\dot{q}_{c w}^{(i)}=y_{0}+m \dot{q}_{c w}^{(r e f)}\right)$

\begin{tabular}{lcrrr}
\hline \hline$P_{s}$ & Calorimeter (i) & $y_{0}$ & $m$ & $\mathrm{R}^{2}$ \\
\hline 15 mbar & $\mathrm{Ag}$ & -165.4 & 1.2659 & 0.9975 \\
$15 \mathrm{mbar}$ & $\mathrm{Qz}$ & -85.32 & 0.5122 & 0.9814 \\
$50 \mathrm{mbar}$ & $\mathrm{Ag}$ & -38.90 & 1.2093 & 0.9974 \\
$50 \mathrm{mbar}$ & $\mathrm{Qz}$ & -52.07 & 0.4374 & 0.9589 \\
$100 \mathrm{mbar}$ & $\mathrm{Ag}$ & 36.88 & 1.0835 & 0.9971 \\
$100 \mathrm{mbar}$ & $\mathrm{Qz}$ & 220.1 & 0.3388 & 0.9849 \\
$200 \mathrm{mbar}$ & $\mathrm{Ag}$ & 12.39 & 1.0574 & 0.9997 \\
$200 \mathrm{mbar}$ & $\mathrm{Qz}$ & 119.5 & 0.4450 & 0.9794 \\
\hline \hline
\end{tabular}

A total of 36 ICP simulations are carried out with CoolFluid to provide the five Non Dimensional Parameters $\Pi_{i}$ required by CERBOULA. Each simulation over an ESA-Standard probe corresponds 
to one test condition. An electric power efficiency of $50 \%$ is assumed [80] to run the simulations. The same isothermal wall at $350 \mathrm{~K}$ is imposed for the three calorimeters because the influence of surface temperature is not relevant for $\Pi_{i}$ computation. Data obtained for $\Pi_{i}$ is collected in Table B.1.

The measured quantities are introduced in CERBOULA, together with $\Pi_{i}$, to rebuild the three $H_{e^{-}} \gamma$ relations for each testing condition. A 7-species air mixture and Gupta et al. 2] chemical model are considered for the process. Note that the choice of the chemical model is critical when applying the Mini-Max because of the higher gas-phase reaction relevance on the measured heat flux at low catalytic conditions, as shown by Krassilchikoff [91] and de Crombrugghe [95]. The fact is, while Dunn \& Kang [4] and Park [3] models give similar results, Gupta et al. [2] leads to a higher $H_{e}$ at low catalytic activities providing a broader range of recombination coefficient data. This is important to obtain an optimal number of test conditions from which it is possible to determine a reference recombination coefficient. The influence of the chemical model was already mentioned by Kovalev [96] when using a similar $H_{e}$ rebuilding procedure.

Intersection results between the $H_{e^{-}} \gamma$ relations are provided in Table 3.4. Unfortunately, catalysis can not be obtained for all plasma conditions either because an intersection of $H_{e, \max }$ with copper's $H_{e^{-}} \gamma$ relation does not take place, or because the rebuilt $H_{e, \max }$ is lower than $H_{e, \min }$, leading to a non-physical testing condition. This limitation is assumed to be related to uncertainties associated with chemical models.

Table 3.4.: Intersections after Mini-Max application

\begin{tabular}{lrrrr}
\hline \hline Test & $\gamma_{\max }$ & $\begin{array}{r}H_{e, \min } \\
\mathrm{MJ} \mathrm{kg}^{-1}\end{array}$ & $\gamma_{\min }$ & $\begin{array}{r}H_{e, \max } \\
\mathrm{MJ} \mathrm{kg}^{-1}\end{array}$ \\
\hline 3a & 0.28630 & 15.32 & 0.02200 & 23.23 \\
4a & 0.14772 & 19.66 & 0.01652 & 37.08 \\
5a & 0.12300 & 24.86 & 0.02022 & 41.26 \\
6a & 0.11610 & 30.82 & 0.02585 & 43.80 \\
7a & 0.11967 & 32.95 & 0.03016 & 44.68 \\
8a & 0.12219 & 36.60 & 0.03596 & 46.05 \\
9a & 0.12352 & 40.88 & 0.04558 & 47.77 \\
\hline 5b & 0.02567 & 24.32 & 0.02164 & 25.05 \\
6b & 0.02787 & 28.64 & 0.02145 & 30.27 \\
7b & 0.03127 & 30.30 & 0.02076 & 33.44 \\
8b & 0.03439 & 32.94 & 0.02140 & 36.96 \\
9b & 0.03827 & 36.61 & 0.02337 & 40.69 \\
\hline 2c & 0.00790 & 15.27 & 0.00097 & 20.87 \\
3c & 0.01143 & 18.17 & 0.00353 & 21.99 \\
4c & 0.01487 & 21.12 & 0.00699 & 23.45 \\
5c & 0.01814 & 24.07 & 0.01298 & 25.06 \\
\hline 1d & 0.00554 & 9.75 & 0.00017 & 12.52 \\
2d & 0.00846 & 13.16 & 0.00286 & 14.45 \\
3d & 0.01103 & 16.15 & 0.01008 & 16.25 \\
\hline \hline
\end{tabular}

Given a range of $\gamma$ for each intersecting plasma condition, the average value is chosen as $\gamma_{r e f}$. Note that, although the $H_{e^{-}} \gamma$ relation is normally presented in logarithmic scale, the logarithmic average 
is not considered here to compute $\gamma_{r e f}$ because of inconsistencies reported by de Crombrugghe [95]. He observed that, under specific Plasmatron settings, the rebuilt $H_{e}$ with a logarithmic averaged $\gamma_{\text {ref }}$ can decrease when a higher heat flux is target on the copper calorimeter. The interval defined by both $\gamma_{r e f}$ and $\gamma_{\min }$ is computed as a percentage over $\gamma_{r e f}$ and named $\gamma^{e r r}$. Its purpose is to provide an idea of the range inside which values of $\gamma_{r e f}$ are obtained, following:

$$
\gamma^{e r r}=\frac{\gamma_{r e f}-\gamma_{\min }}{\gamma_{r e f}}
$$

The reference catalysis of each testing condition allows the rebuilding of $H_{e}$ with CERBOULA, which is also associated to an interval defined by both $H_{e, \min }$ and $H_{e, \max }$. This range is given also as a percentage over the rebuilt $H_{e}$, defining $H_{e}^{e r r}$ as:

$$
H_{e}^{e r r}=\frac{H_{e, \max }-H_{e, \min }}{H_{e}}
$$

Results of $\gamma_{r e f}$ and $H_{e}$ are summarized in Table 3.5 .

Table 3.5.: Definition of $\gamma_{r e f}$ and the rebuilt $H_{e}$ after Mini-Max application

\begin{tabular}{crrrr}
\hline \hline Test & $\gamma_{\text {ref }}$ & $\begin{array}{r}\gamma^{\text {err }} \\
\%\end{array}$ & $\begin{array}{r}H_{e} \\
\mathrm{MJ} \mathrm{kg}^{-1}\end{array}$ & $\begin{array}{r}H_{e}^{\text {err }} \\
\%\end{array}$ \\
\hline 3a & 0.15415 & 85.73 & 15.84 & 49.92 \\
4a & 0.08212 & 79.89 & 21.11 & 82.52 \\
5a & 0.07161 & 71.76 & 27.26 & 60.14 \\
6a & 0.07098 & 63.57 & 34.02 & 38.15 \\
7a & 0.07491 & 59.74 & 36.41 & 32.22 \\
8a & 0.07908 & 54.53 & 39.82 & 23.75 \\
9a & 0.08455 & 46.09 & 43.19 & 15.96 \\
\hline 5b & 0.02366 & 8.51 & 24.60 & 2.97 \\
6b & 0.02466 & 13.01 & 29.31 & 5.55 \\
7b & 0.02602 & 20.21 & 31.51 & 9.98 \\
8b & 0.02790 & 23.28 & 34.43 & 11.68 \\
9b & 0.03082 & 24.17 & 38.30 & 10.63 \\
\hline 2c & 0.00443 & 78.17 & 16.43 & 34.12 \\
3c & 0.00748 & 52.82 & 19.15 & 19.93 \\
4c & 0.01093 & 36.06 & 21.90 & 10.64 \\
5c & 0.01556 & 16.58 & 24.49 & 4.05 \\
\hline 1d & 0.00285 & 93.89 & 10.28 & 27.00 \\
2d & 0.00566 & 49.45 & 13.54 & 9.49 \\
3d & 0.01056 & 4.48 & 16.20 & 0.60 \\
\hline \hline
\end{tabular}

Remark that the margins in enthalpy and catalysis only assess the quality of the intersection between $H_{e^{-}} \gamma$ relations across the three calorimeters, and they should not be seen as an uncertainty quantification of the measured variables. A thorough UQ analysis of the Mini-Max test campaign is encouraged in the future using Bayesian inference. The advantage of this method lies on the fact that it would use experimental data from the system, not only to estimate the most probable $\gamma_{r e f}$ 
and its uncertainty, but also to assess the uncertainties of other assumed quantities such as surface temperature and catalysis on both silver and Quartz calorimeters. Some preliminary results of UQ on the Mini-Max report an uncertainty of $\pm 35 \%$ around $\gamma_{r e f}$ (to be published).

\subsubsection{Analysis at $15 \mathrm{mbar}$}

The Mini-Max method provides the recombination coefficient on copper for a wide range of data at 15 mbar. This is due to the high sensitivity of $H_{e}$ with $\gamma$ at low pressures. Results are plotted in Fig. 3.11. The large values of $\gamma^{e r r}$ at low powers show that $\gamma_{r e f}$ falls in the flat region of the $H_{e^{-}} \gamma$ relation. This is confirmed by the high catalycity reported for condition 3a. Then, the $\gamma_{r e f}$ determination improves with the increase of power because of the higher atomic concentration of nitrogen at the outer edge of the boundary layer, which makes heat flux measurements more sensitive to catalysis and $\gamma_{r e f}$ falls in a steeper region of the $H_{e^{-}} \gamma$ relation. Although $\gamma^{e r r}$ is quite large for most of the tests, this does not turn into a limitation for $H_{e}$ rebuilding with CERBOULA at high powers, as reported by condition 9a.

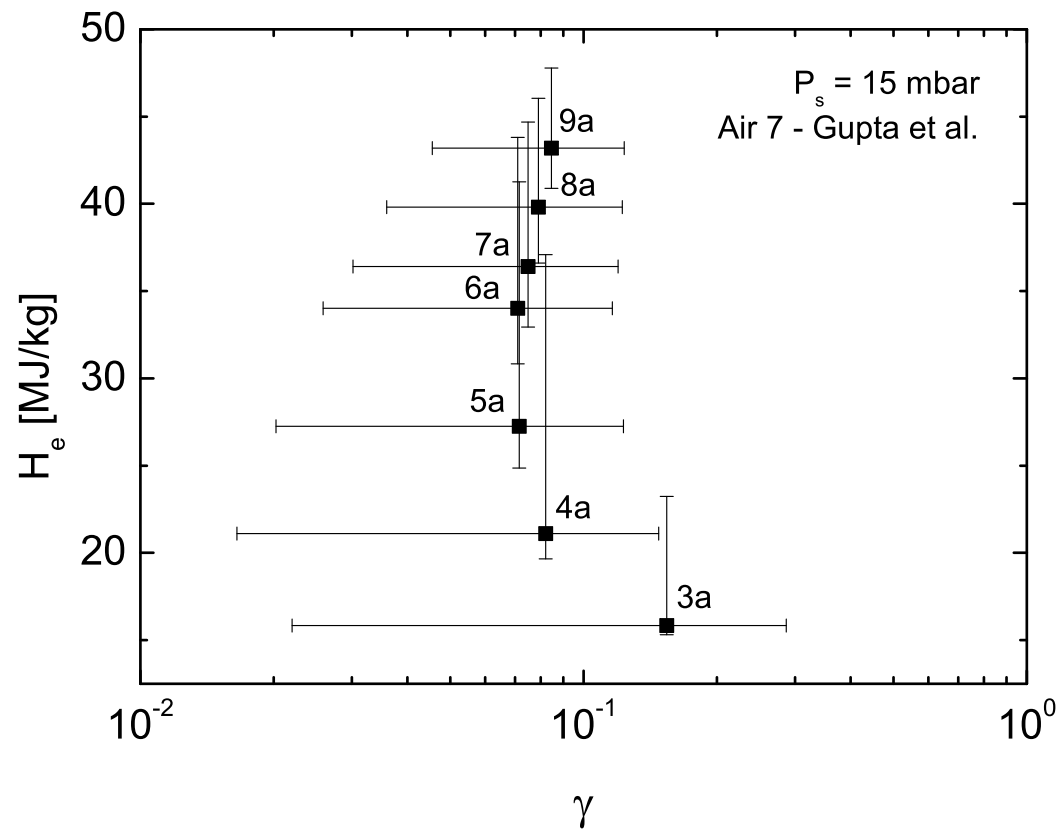

Figure 3.11.: Reference recombination coefficient at 15 mbar.

\subsubsection{Analysis at 50 mbar}

Results of the Mini-Max campaign at 50 mbar are shown in Fig. 3.12. The obtained recombination coefficients remaining almost constant with power, they are reduced with respect to those obtained at 15 mbar. This is due to the higher recombination in the gas expected at higher pressures, making the measured heat flux less sensitive to catalytic reactions. Narrower ranges for both $\gamma$ and $H_{e}$ are obtained at 50 mbar. Contrary to what is observed at 15 mbar, the $\gamma_{r e f}$ determination improves under low power conditions. This is consequence of the low enthalpy range provided by $H_{e, \min }$ on 
silver and $H_{e, \max }$ on Quartz calorimeters, and the fact that their intersection with copper's $H_{e^{-}} \gamma$ relation falls in a steep region of the curve.

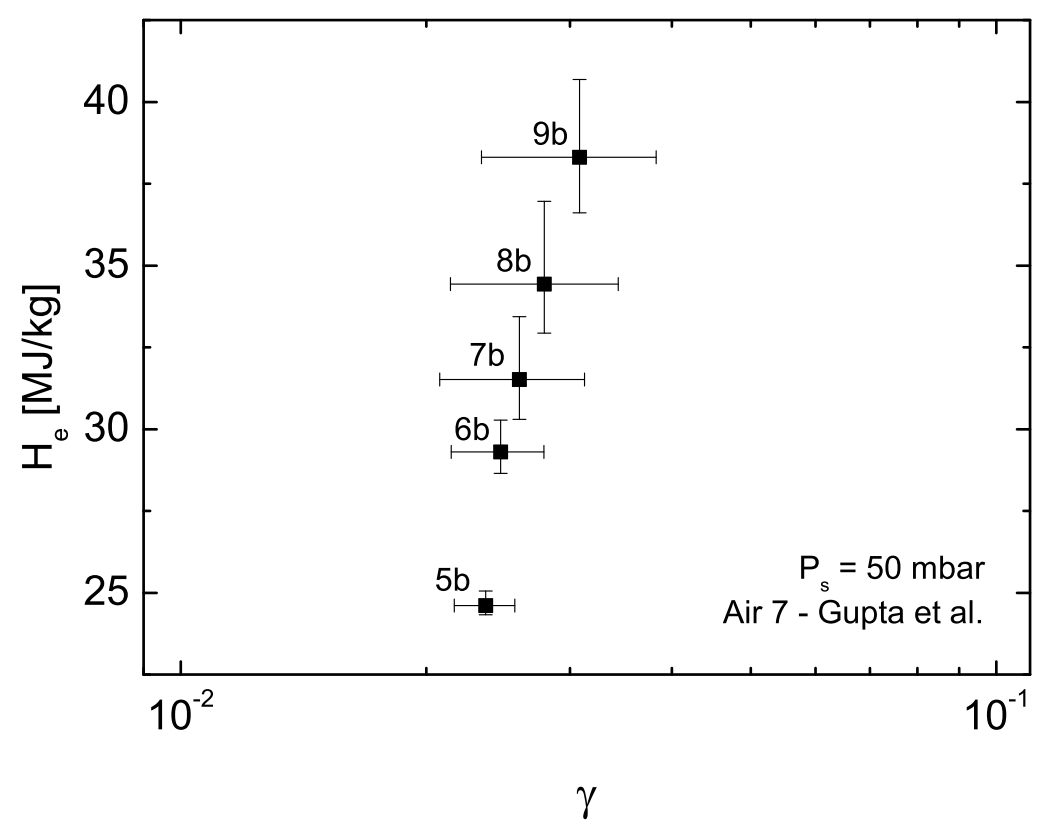

Figure 3.12.: Reference recombination coefficient at 50 mbar.

\subsubsection{Analysis at $100 \mathrm{mbar}$}

The number of successful test cases for $\gamma_{r e f}$ determination is reduced with the increase in pressure. This is shown by comparing the results at 100 mbar in Fig. 3.13 with the previous ones. What happens is that the increase of gas-phase recombination with higher pressures reduces the sensitivity of $H_{e}$ with $\gamma$ to the point where $H_{e^{-}} \gamma$ relations do not intersect anymore, leading to only a few number of successful results. As observed at $50 \mathrm{mbar}$, the recombination coefficients obtained are nearly independent of power. Remark also that power levels from which a $\gamma_{\text {ref }}$ is obtained are lower than those at 15 and 50 mbar. However, both $\gamma^{e r r}$ and $H_{e}^{e r r}$ are reduced with the increase in power, driving the optimal rebuilding condition to intermediate power settings (tests $4 \mathrm{c}$ and $5 \mathrm{c}$ ). Note also that $\gamma_{r e f}$ values are significantly lower than for 15 and 50 mbar, leading to the conclusion that $\gamma_{r e f}$ is, indeed, pressure dependent.

\subsubsection{Analysis at $200 \mathrm{mbar}$}

Only three test conditions provide $\gamma_{\text {ref }}$ measurements at 200 mbar. They correspond to the lowest power settings, from $1 \mathrm{~d}$ to $3 \mathrm{~d}$, as shown in Fig. 3.14. The power dependence of $\gamma_{r e f}$ is higher than on previous pressure conditions, although the effect remains limited. The reduction of the recombination coefficient with pressure is not so clear when passing from 100 to 200 mbar. In fact, $\gamma_{r e f}$ for $3 \mathrm{~d}$ is quite close to the reported for $4 \mathrm{c}$. The values of $H_{e}^{\text {err }}$ are small enough to consider 


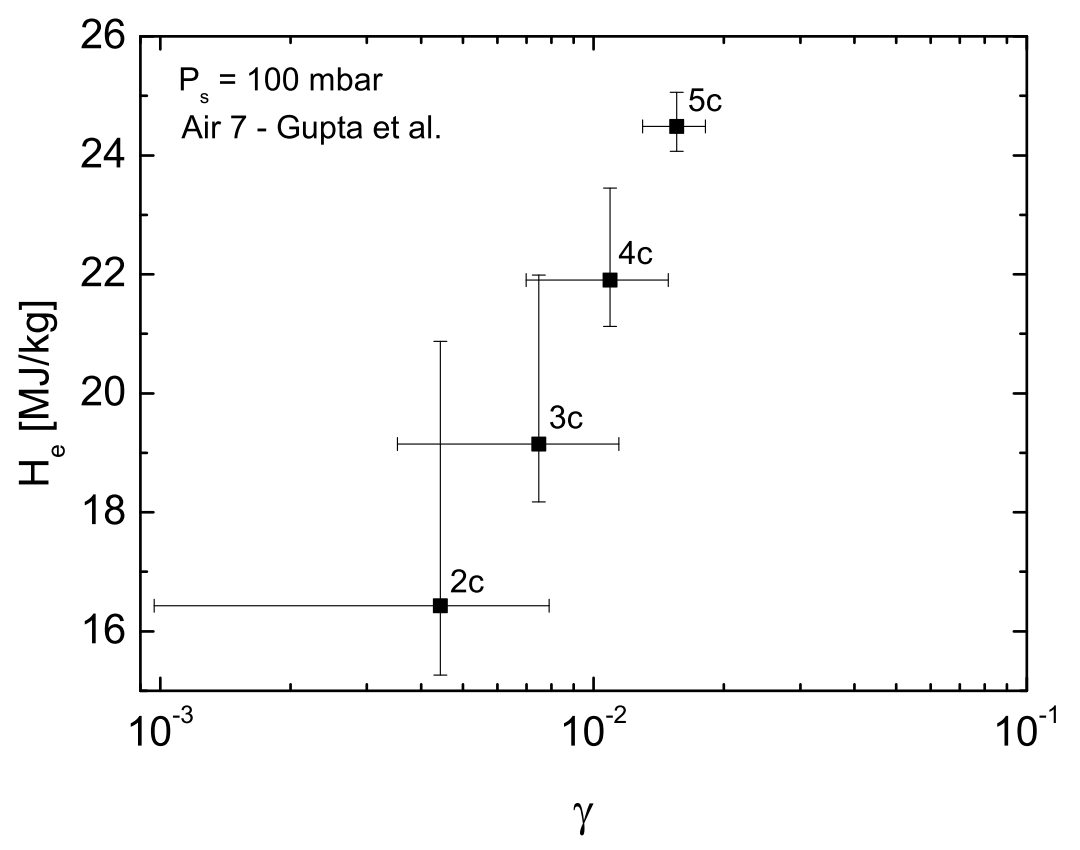

Figure 3.13.: Reference recombination coefficient at 100 mbar.

the $\gamma_{r e f}$ measure appropriate for $H_{e}$ rebuilding. Again, this is a consequence of the low sensitivity of $H_{e}$ with respect to $\gamma$ at high pressures.

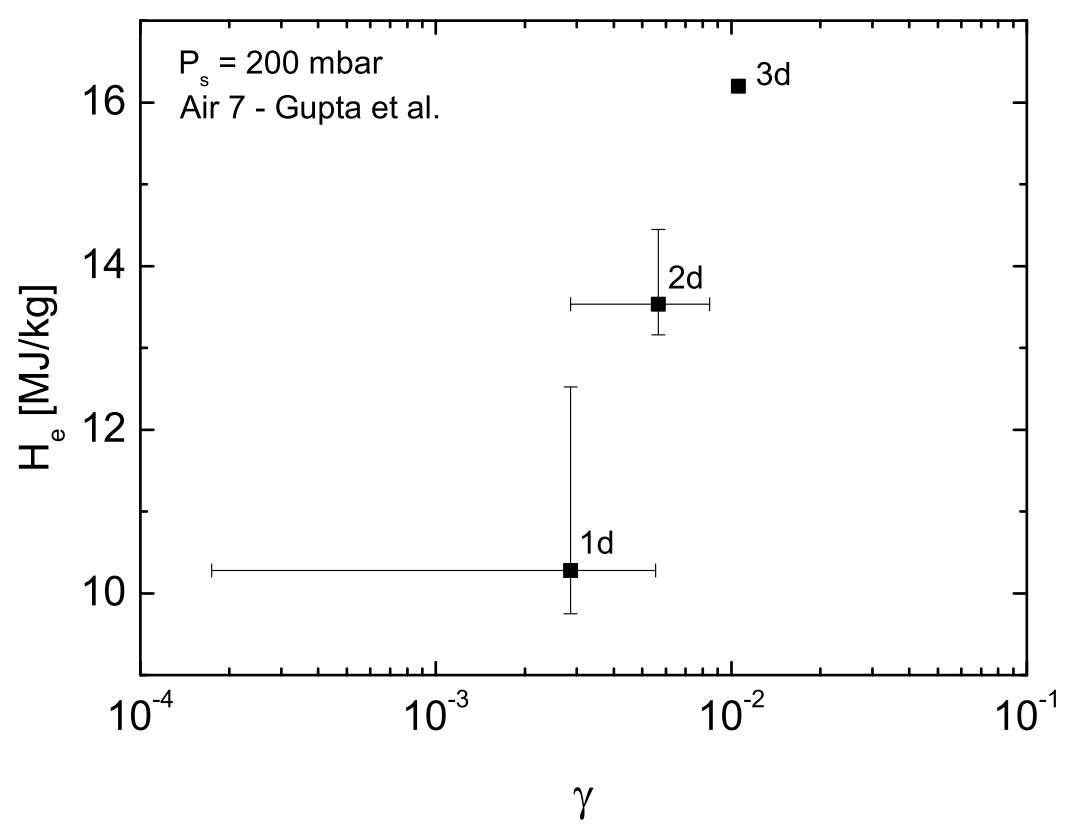

Figure 3.14.: Reference recombination coefficient at 200 mbar. 


\subsection{The Plasmatron reference catalytic model}

This experimental campaign shows that, while the measurement of $\gamma_{r e f}$ is not always accurate, this does not necessarily mean that the rebuilding method is not precise enough to be used for Plasmatron characterization. In fact, this is an intrinsic effect given the different sensitivities of the $H_{e^{-}} \gamma$ relation through the domain from non-catalytic to full-catalytic. However, one should be aware that not all testing conditions are suited for the rebuilding method, especially under low power conditions, where nitrogen dissociation is low.

Note also that, while the recombination coefficient does not depend strongly on $H_{e}$, it is significantly affected by static pressure $P_{s}$. This is the reason why, for practical reasons, the use of a reference recombination coefficient $\bar{\gamma}_{\text {ref }}$ averaged across the different power levels for each pressure condition is proposed. Thus, one can define the following pressure dependent $\gamma$-model on copper for the Plasmatron ${ }^{3}$ (Fig. 3.15):

$$
\bar{\gamma}_{r e f}\left(P_{s}\right)= \begin{cases}0.07738 & \text { if } P_{s}=15 \mathrm{mbar} \\ 0.02661 & \text { if } P_{s}=50 \mathrm{mbar} \\ 0.00960 & \text { if } P_{s}=100 \mathrm{mbar} \\ 0.00636 & \text { if } P_{s}=200 \mathrm{mbar}\end{cases}
$$

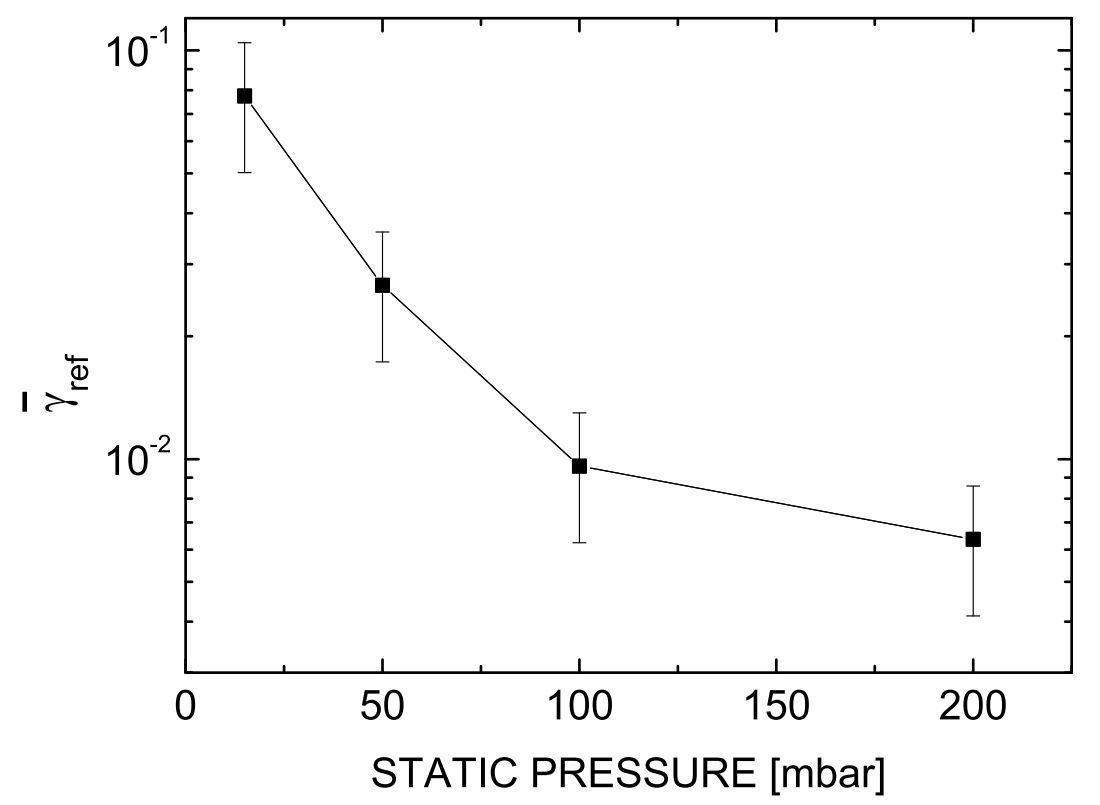

Figure 3.15.: Reference recombination coefficient model on copper for enthalpy rebuilding in the Plasmatron. A $\pm 35 \%$ of uncertainty is assumed based on preliminary UQ analysis (to be published).

One important remark is that, although the effect of pressure was already observed by Panerai [11] and by Esser [97], this is not really consistent with the catalytic model that is used in NEBOULA for

\footnotetext{
${ }^{3}$ Tests $3 \mathrm{a}, 4 \mathrm{a}$ and $5 \mathrm{a}$ are neglected from the average because the $H_{e}^{\text {err }}$ associated to these measurements is higher than $40 \%$.
} 
the enthalpy rebuilding process, which should be only function of $T_{w}$. This aspect will be addressed in Ch. 4.

As final remark on the Mini-Max, if one intends to rebuild $H_{e}$ using the $\gamma_{r e f}$-model, it is highly recommended either to check the database provided above before testing or to apply the Mini-Max methodology at the desired Plasmatron conditions prior the testing campaign. 


\section{Chapter 4.}

\section{Catalysis in Different Flow Configurations}

The procedure to determine the recombination coefficient of a catalytic sample in the VKI-Plasmatron facility relies on both a heat flux measurement and an accurate estimation of the boundary layer outer edge enthalpy $H_{e}$, for given static pressure $P_{s}$, gas mass flow $\dot{m}$ and coil power $P W$. This means that an influence of these variables could be expected over the determined recombination coefficient. Indeed, the $\gamma_{r e f}$ modelling presented in the previous chapter shows how the recombination coefficient on copper at $\approx 350 \mathrm{~K}$ is strongly influenced by $P_{s}$.

The effect of pressure is neglected in most of the literature because the assumption of first order reaction $(n=1)$ on the catalytic surface is made for macroscopic models (see Sec. 2.1.1), leading to a $\gamma$ that only depends on $T_{w}$. Remark that other models exist for finite rate catalysis, but they require a lot of data to be validated and are not always practical for the design of re-entry vehicles, as discussed in Sec. 2.1.2. To illustrate how pressure affects the recombination coefficient determination, and to support results obtained in the previous chapter, the $H_{e^{-}} \gamma$ relation for a range of $\gamma$ spanning from non-catalytic to fully-catalytic walls is provided in Fig. 4.1. Values are given for three different static pressures and constant cold wall heat flux $\left(\dot{q}_{c w}=900 \mathrm{~kW} / \mathrm{m}^{2}\right)$. It is seen that increasing pressure makes the rebuilt enthalpy values with the non-equilibrium solver less sensitive to catalytic reactions, leading to lower recombination coefficients.

It is known that a pressure variation could significantly change the diffusion properties of the boundary layer. Considering gas chemistry, for instance, a higher pressure increases the number of collisions between particles, meaning that more atomic recombination could be expected in the bulk of the boundary layer. This effect is shown in Fig. 4.2, where the N- and O-atom profiles are numerically generated over the Standard probe used in Sec. 3.1 under non-catalytic conditions. A significant amount of nitrogen recombination is expected during a Plasmatron test for pressures higher than 15 mbar, while the boundary layer remains almost frozen for oxygen recombination. Remark that, modifying the diffusion properties of the boundary layer could also have an influence on the boundary layer thickness $\delta$, so the experimentally determined recombination coefficient might appear also dependent on either the probe geometry or the flow configuration. 


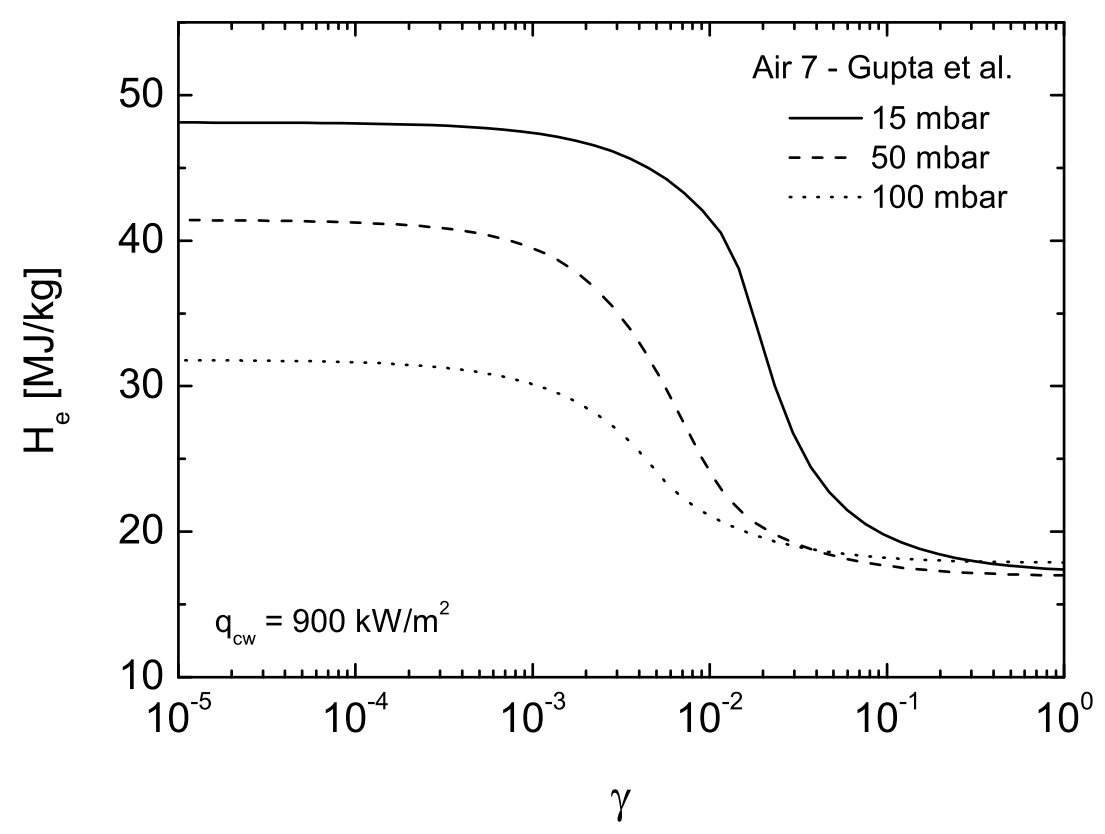

Figure 4.1.: Static pressure effect on the $H_{e^{-}} \gamma$ relation built with CERBOULA.

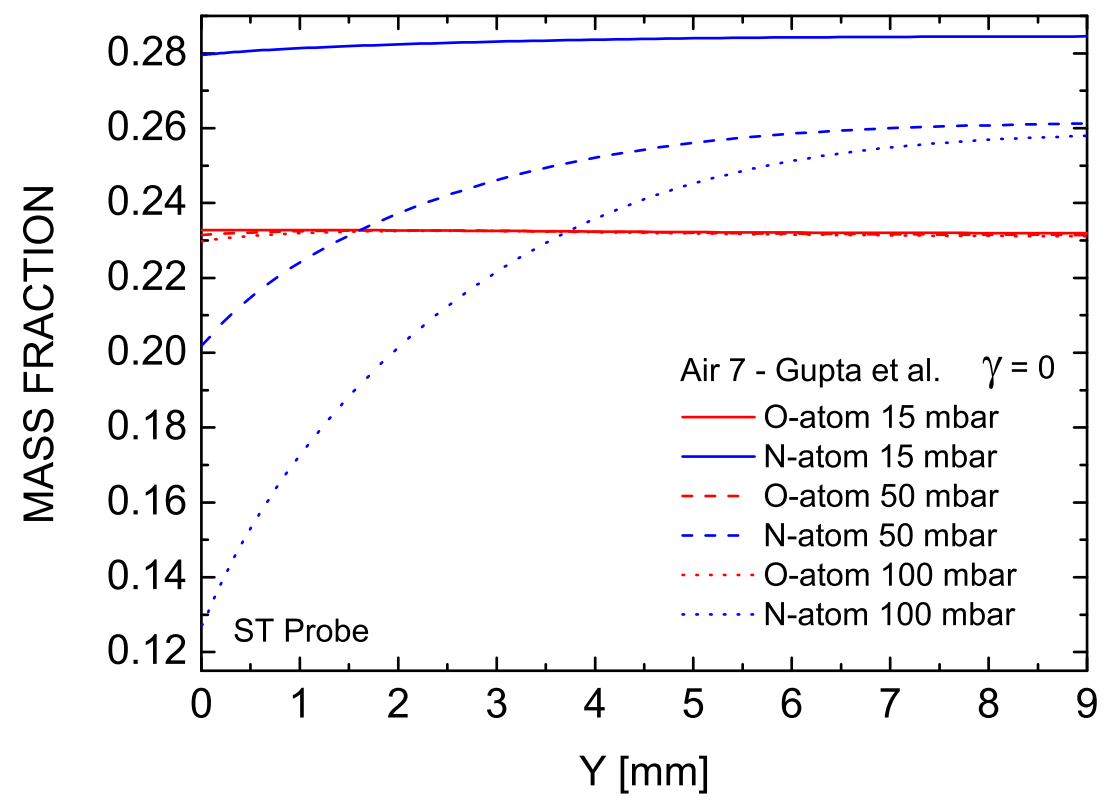

Figure 4.2.: Static pressure effect on the N- and O-atom profiles under non-catalytic conditions.

A simple dimensional analysis can be carried out to explain the role of the flow configuration on the chemical non-equilibrium characteristics of the boundary layer. For that, the species conservation equation should be taken under consideration. For sake of simplicity, Eq. 1.3 will be considered in steady state and non-compressible form, leading to: 


$$
\vec{V} \cdot \nabla \rho_{i}+\nabla \cdot \vec{J}_{i}=\dot{\omega}_{i}
$$

The diffusion fluxes will be described by Fick's law $\left(\vec{J}_{i}=-\rho D \nabla c_{i}\right)$. Assuming also first order reactions, the production rate reads $\dot{\omega}_{i}=k_{f} \rho_{i}$. Then, the boundary condition at the surface of a catalytic wall can be written as:

$$
\left.\rho_{w} D \nabla c_{i}\right|_{w}=k_{w}\left(\rho c_{i}\right)_{w}
$$

If one writes both Eq. 4.1 and Eq. 4.2 in dimensionless form considering the stagnation point boundary layer thickness $\delta$ as the characteristic length of the problem, and the diffusion velocity defined by $D / \delta$ as the reference speed of the problem, the following is obtained:

$$
\begin{gathered}
\overrightarrow{\tilde{V}} \cdot \tilde{\nabla}\left(\tilde{\rho} c_{i}\right)+\tilde{\rho} \tilde{\nabla} c_{i}=D a_{g}\left(\tilde{\rho} c_{i}\right) \\
\left.\tilde{\rho}_{w} \tilde{\nabla} c_{i}\right|_{w}=D a_{w}\left(\tilde{\rho} c_{i}\right)_{w}
\end{gathered}
$$

Note that two Damköhler numbers appear in the source terms (right hand sides). One is known as the gas-phase Damköhler number $D a_{g}$ and the other as the wall Damköhler number $D a_{w}$. They read?

$$
\begin{aligned}
& D a_{g}=\frac{k_{f} \delta^{2}}{D} \\
& D a_{w}=\frac{k_{w} \delta}{D}
\end{aligned}
$$

A Damköhler number is always defined as the ratio between a characteristic time of the flow and a characteristic time of the chemistry $D a=\tau_{\text {flow }} / \tau_{\text {chem }}$. Regarding the gas phase, when the flow velocity is much faster than the reaction rates, the $D a_{g} \rightarrow 0$ and the source term can be neglected. In such situation, the boundary layer can be considered without gas-reactions and assumed in frozen regime. Conversely, when chemistry is much faster than the flow, the $D a_{g} \rightarrow \infty$, and the boundary layer is said to be in chemical equilibrium. Remark that, in this last situation, species composition can be defined through temperature and pressure only.

Similarly, when considering the reactions at the wall in Eq. 4.4, there is a mass balance imposing that the species diffusing towards/from the surface should be compensated by the species depleted/generated by the catalytic reactions on the surface. Therefore, the situations where $D a_{w} \rightarrow 0$ are known as reaction limited regimes and they mean that the diffusive properties of the gas overcome the wall reactive capabilities, so the catalytic production of species can be disregarded (independently of the wall's reaction rate constant $k_{w}$ ) from the equation. On the contrary, the situations where $D a_{w} \rightarrow \infty$ are known as diffusion limited regimes, which means that the reactions at the surface are so fast that the diffusion rate of the gas is insufficient to sustain the chemical reactions at the wall, so the production rate reaches its upper limit (also independently of $k_{w}$ ).

\footnotetext{
${ }^{1}$ Remark that the units of a gas-phase reaction rate constant $\left(\mathrm{s}^{-1}\right)$ differ from those of a reaction rate constant on a surface $\left(\mathrm{ms}^{-1}\right)$.
} 
It is important to not confuse the reaction limited regime with the non-catalytic boundary condition, and the diffusion limited regime with the full-catalytic condition. While the catalytic condition is strictly related to the rate constant $k_{w}$ (and hence to $\gamma$ through Eq.2.3), the reaction and diffusion limited regimes are defined by the combination of the wall catalytic conditions with the fluid diffusive properties. Thus, a finite catalytic wall can generate a diffusion limited regime under specific diffusion characteristics of the gas.

As mentioned in Sec. 2.1.1, Goulard explained that an experimental determination of recombination coefficients is possible in binary mixtures with no gas-phase atomic recombination (frozen flow). Indeed, these ideal testing conditions could be easily obtained by Balat-Pichelin et al. 43, 46, 48, and Driver [88, 98] due to the low pressure environments provided by their diffusion reactors (only a few pascals). However, the measurement of $\gamma$ in larger plasma facilities is also of interest because relevant flight conditions need to be simulated. Since the peak heating conditions are reached under higher pressure conditions, plasma wind tunnels such as the VKI-Plasmatron are designed to operate at pressures larger than those found in diffusion reactors, meaning that the presence of gasphase atomic recombination is likely to occur and frozen flow conditions might not be met during tests. These reacting boundary layer conditions are inherent to larger facilities dedicated to TPS catalysis determination, and their understanding is of major relevance for both the ground-to-flight extrapolation activities being developed at VKI and the validation of GSI models.

This chapter contributes to the understanding of the chemical non-equilibrium implications on the experimental determination of the recombination coefficient. Its purpose is to demonstrate that a coupling exists between the gas-phase chemistry and the recombination coefficient that is measured in the Plasmatron. To this end, a theoretical discussion explaining why this coupling could appear (beyond a pressure effect) is provided. This is complemented with a parametric analysis using the in-house developed numerical tools dedicated to the reconstruction of the flow environment in the Plasmatron test chamber and from which catalysis is determined. Experimental results for different probe configurations are provided, so that non-equilibrium effects on catalytic measurements can be better understood.

\subsection{On the catalysis determination in non-equilibrium flows}

To illustrate the problem regarding catalytic measurements under non-equilibrium boundary layers, it is worth to consider the work of Rosner [99], in which Broadwell's conductivity cell model [100] is generalized to include first order atom recombination at a cold surface of arbitrary catalytic activity. The flow within two parallel plates separated by a distance $\delta$ and enclosing a partially dissociated gas is analyzed. One of the plates is hot and under LTE conditions, while the other one is cold and with finite catalytic properties.

Three non-dimensional parameters appear in the problem: $D a_{g}, D a_{w}$ and $H . D a_{g}$ is defined here as the ratio between the characteristic diffusion time $\delta^{2} / D_{12}$ and the characteristic chemical relaxation time $\tau_{c h e m}$. $D a_{w}$ introduces the kinetics at the cold wall through $k_{w}$. The parameter $H$ is a measure of the maximum possible enthalpy change $\Delta h_{\text {chem,eq }}$ across the cell as compared to the enthalpy change under frozen conditions $\Delta h_{f r}$ for the given temperature difference $T_{\text {hot }}-T_{\text {cold }}$ imposed by the two plates. $H$ includes as multiplier a recovery factor $r_{D}$ for chemical energy 
that is function of the Lewis number for stagnant flows. The Nusselt number $(N u)$ based on the temperature change between the plates across the film thickness $\delta$ is computed with Eq. 4.7, where $\phi$ is defined as in Eq. 4.8 and $\xi=\sqrt{D a_{g}(1+H)}$.

$$
\begin{gathered}
N u=1+\phi H \\
\phi=1-\left\{1+D a_{w}-\frac{1-\xi \operatorname{coth} \xi}{1+H}\right\}^{-1}
\end{gathered}
$$

The surface $N u=f\left(D a_{g}, D a_{w}\right)$ provided by Rosner is shown in Fig. 4.3 for $H=9$. As expected, the heat flux transferred to the cold wall increases with both Damköhler numbers. However, one may notice that for large $D a_{g}$, there are less atoms available close to the surface due to the presence of gas-phase recombination, leading to a heat flux that is less sensitive to the wall catalytic reactions. This fact could have an important consequence when determining a catalytic recombination coefficient based on calorimetric measurements in plasma wind tunnels because uncertainties associated with measuring instruments, data processing and chemical models may lead to an experimentally determined catalytic activity that is influenced by many flow parameters instead of only the surface temperature. Indeed, this is the reason why the preferred conditions for catalysis determination are under very low pressure environments, where collisions between atoms is unlikely to occur and the frozen flow conditions are achieved.

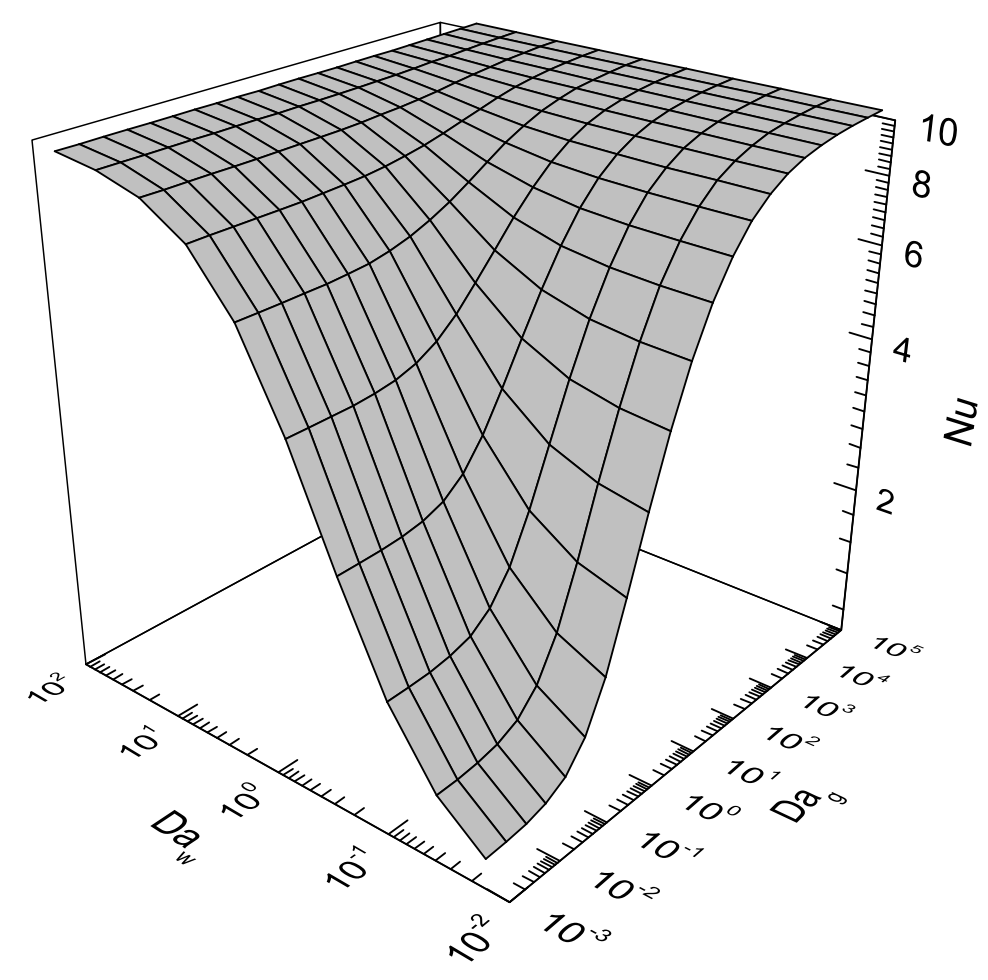

Figure 4.3.: $N u$ number as a function of $D a_{g}$ and $D a_{w}$ for $H=9$, as proposed by Rosner [99].

Therefore, the experimentally determined recombination coefficient could be a function of the particular combination of $D a_{g}, D a_{w}, N u$ and $H$, corresponding to each test condition. This means 
that there is a role of the species diffusion inside the boundary layer, ruled by the relevance of both gas-phase and surface reactions with respect to the flow velocity, that can affect the chemical contribution to the heat flux. As a consequence, any recombination coefficient determined through a heat flux measurement could seem influenced by the boundary layer diffusion characteristics.

\subsubsection{Parametric analysis with NEBOULA}

NEBOULA can be used in order to evaluate the effect of both the gas-phase Damköhler number and the catalytic recombination coefficient on the numerical heat flux $\dot{q}_{\text {num }}$, under representative plasma wind tunnel testing conditions. To this end, $D a_{g}$ is varied by adjusting pressure from 10 to $500 \mathrm{mbar}$, and $\gamma$ ranges from $10^{-5}$ (non-catalytic) to 1 (full-catalytic). The rest of the input parameters are kept constant. The outer edge and the wall temperatures are fixed to $8000 \mathrm{~K}$ and $350 \mathrm{~K}$, respectively. The velocity gradient at the outer edge is set to $3000 \mathrm{~s}^{-1}$. Density, viscosity and species mass fractions are imposed assuming LTE for each $T_{e}$ and $P_{s}$ condition. The boundary layer thickness is fixed at $1 \mathrm{~cm}$ as representative of the Standard probe, and the free-stream velocity of the plasma is set to $100 \mathrm{~m} / \mathrm{s}$. Gupta et al. [2] chemical model is used for the gas-phase reaction rates and the computation of the chemical relaxation time $\tau_{\text {chem }}$.

Remark that the gas-phase Damköhler number is not universally defined due to the lack of knowledge on the characteristic relaxation time of the chemistry. A method to estimate $\tau_{c h e m}$ is proposed below, considering the dissociation from a molecule in the form of $\mathrm{X}_{2}$ to atomic $\mathrm{X}$ using the collision partner M:

$$
\mathrm{X}_{2}+\mathrm{M} \underset{k_{b}}{\stackrel{k_{f}}{\rightleftarrows}} 2 \mathrm{X}+\mathrm{M}
$$

The dissociation rate of $\mathrm{X}_{2}$ can be expressed as:

$$
\frac{\partial\left[\mathrm{X}_{2}\right]}{\partial t}=k_{b}[\mathrm{X}]^{2}[\mathrm{M}]-k_{f}\left[\mathrm{X}_{2}\right][\mathrm{M}]
$$

where $\left[\mathrm{X}_{2}\right],[\mathrm{X}]$ and $[\mathrm{M}]$ are the molar concentrations of molecules, atoms and collision partners, respectively. Considering $\chi$ as the change in molar concentration of molecules to reach chemical relaxation, and assumed to be a small perturbation from the equilibrium state, one could write the atomic molar concentration as $[\mathrm{X}]=[\mathrm{X}]_{E q}+2 \chi$ and the molecular molar concentration as $\left[\mathrm{X}_{2}\right]=\left[\mathrm{X}_{2}\right]_{E q}-\chi$. Introducing these definitions into Eq. 4.9 , considering the chemical equilibrium condition stated as:

$$
\frac{\partial\left[\mathrm{X}_{2}\right]_{E q}}{\partial t}=k_{b}[\mathrm{X}]_{E q}^{2}[\mathrm{M}]-k_{f}\left[\mathrm{X}_{2}\right]_{E q}[\mathrm{M}]=0
$$

and neglecting second order terms, it is possible to find the rate equation for $\chi$ as follows:

$$
\frac{\partial \chi}{\partial t}=-\left(4 k_{b}[\mathrm{X}]_{E q}+k_{f}\right)[\mathrm{M}] \chi
$$

Finally, the chemical relaxation time can be expressed as:

$$
\tau_{\text {chem }}=\frac{1}{\left(4 k_{b}[\mathrm{X}]_{E q}+k_{f}\right)[\mathrm{M}]}
$$


And the gas-phase Damköhler number reads:

$$
D a_{g}=\frac{[\mathrm{M}] \delta^{2}}{D}\left(4 k_{b}[\mathrm{X}]_{E q}+k_{f}\right)
$$

Using this definition of the $D a_{g}$ in NEBOULA, the parametric analysis leads to the Nusselt number $\left(N u=\dot{q}_{\text {num }} \delta / \lambda\left(T_{e}-T_{w}\right)\right)$ distribution shown in Fig. 4.4. Note that the $\mathrm{N}_{2}$ dissociation is considered as the most representative reaction in the flow for the calculation of $D a_{g}$ at the outer edge. Such computation is carried out considering atomic $\mathrm{N}$ as the collision partner in the chemical model proposed by Gupta et al. 2], imposing $k_{f}=4.15 \times 10^{16} \mathrm{~T}^{-1.5} \mathrm{e}^{-1.131 \times 10^{5} / \mathrm{T}} \mathrm{m}^{3} / \mathrm{mole} \mathrm{s}$ and $k_{b}=$ $2.32 \times 10^{15} \mathrm{~T}^{-1.5} \mathrm{~m}^{6} / \mathrm{mole}^{2} \mathrm{~s}$. The diffusion coefficient is provided by Eq. 3.203 .21 using the collision integrals $\bar{\Omega}_{i j}^{11}$ from Capitelli et al. [101].

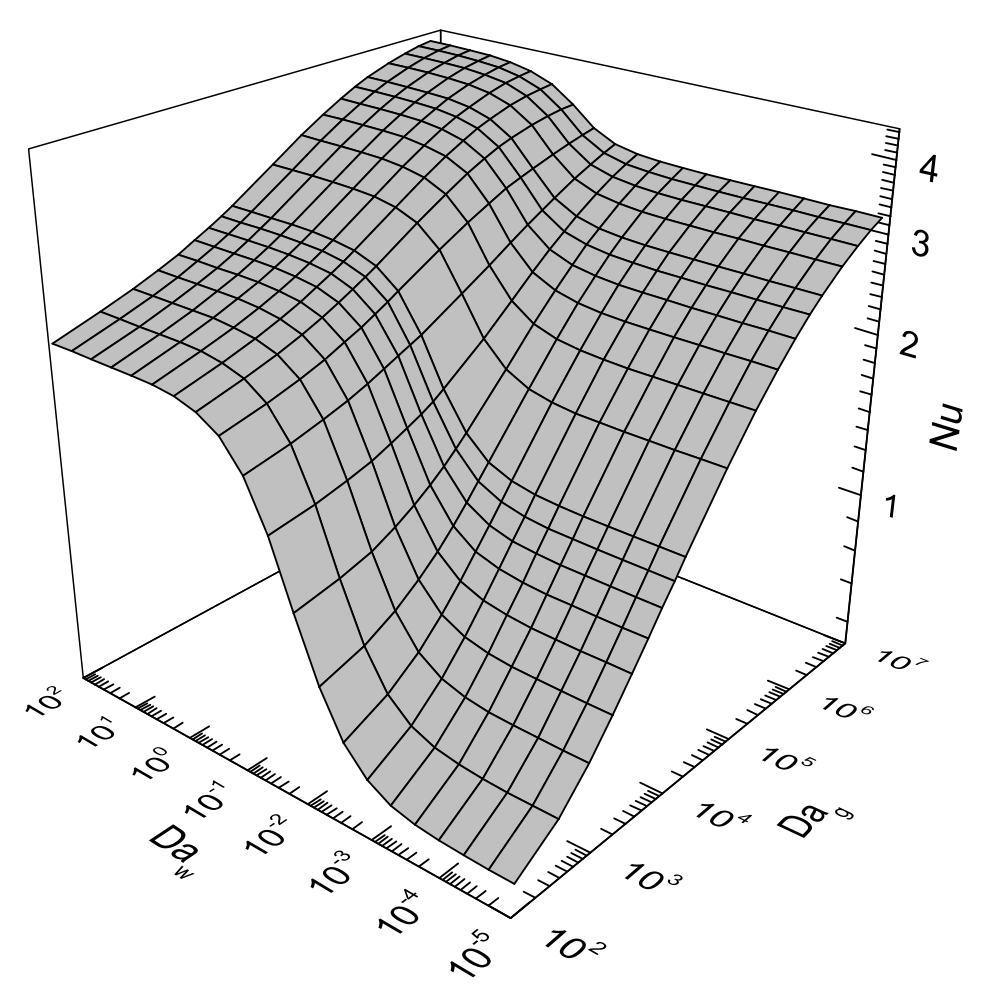

Figure 4.4.: $N u$ number as function of $D a_{g}$ and $D a_{w}$, computed with NEBOULA.

The analysis confirms that similar coupling effects between the gas-phase reactions and the catalytic recombination on the surface should be expected in the non-equilibrium boundary layers developed at stagnation point under typical Plasmatron operating conditions. It is important to take this into account when quantifying surface catalysis on a TPS material sample using both a heat flux measurement and a non-equilibrium boundary layer code. Although the theoretical and numerical analysis show the same trend, it is worth noting that neither the full-equilibrium boundary layer nor the diffusion limited regimes are reached in this parametric analysis. This means that such flow configurations are quite difficult to be achieved in the chamber of a plasma wind tunnel for any of the testing conditions that could be imposed.

In practice, however, the relevant variables to be measured in the test facility are the heat flux and the recombination coefficients. This is the reason why it is also of interest to see the relation 
of these variables for different $D a_{g}$, as shown in Fig. 4.5. Since pressure influences the amount of heat flux even at the full-catalytic wall, the relative heat flux with respect to the full-catalytic case is considered here. Indeed, the coupling effect between volume and surface reactions persists and the sensitivity of the heat flux on $\gamma$ is reduced when $D a_{g}$ is increased.

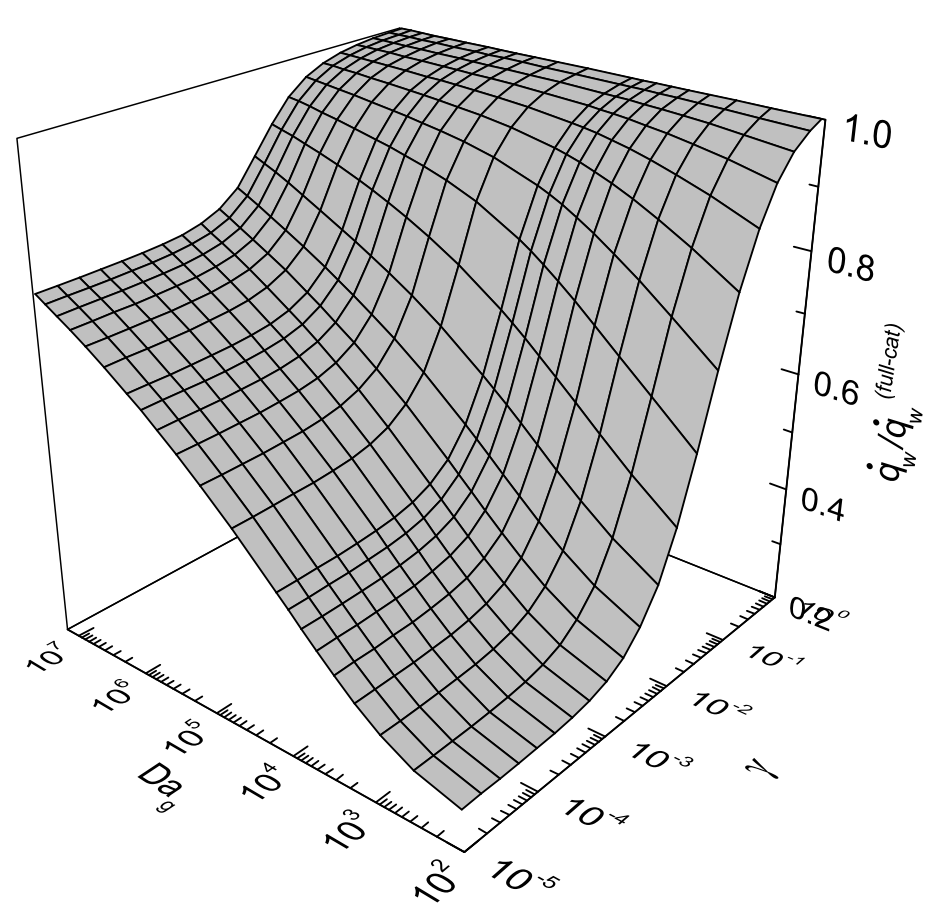

Figure 4.5.: Relative heat flux as function of $D a_{g}$ and $\gamma$, computed with NEBOULA.

Therefore, one could expect an influence of the gas-phase chemistry when trying to measure the recombination coefficient by means of an energy balance at the wall of a sample or calorimetric heat flux measurement. In fact, the existence of such influence was already mentioned by Fay during a discussion about the work of Busing [102] on the measurement of recombination coefficients, quoting: "In the author's experiments, an increase in pressure should cause the disappearance of the catalytic effect when the boundary layer achieves equilibrium. Such experiment would be extremely useful in recombination studies".

This effect is expected in the VKI-Plasmatron facility, which can work at several pressure levels and with different probe configurations. The experimental results shown in the following sections try to provide an evidence of how different levels of non-equilibrium in the boundary layer can give rise, indeed, to different catalytic measurements.

\subsection{Testing configurations at the VKI-Plasmatron}

Results of Sec. 3.4 already show an influence of pressure on the experimentally determined recombination coefficient. This chapter tries to go further on the non-equilibrium analysis by modifying the boundary layer characteristics, not changing the test chamber pressure, but with different probe 
geometries and flow configurations. There are different strategies to change the non-equilibrium properties of the flow in front of a calorimeter. The most intuitive is by changing the dimensions of the stagnation-point holders in order to modify the thickness of the boundary layer. Indeed, this is expected to modify the gas composition close to the calorimeter, making the heat flux measurement more or less sensitive to catalysis. Another option is by testing with an off-stagnation point configuration, using a flat plate holder and a new linear calorimeter concept. Both configurations are presented below.

\subsubsection{Stagnation point: the Damköhler probes}

The three Damköhler probes shown in Fig. 4.6 are available in the VKI to take stagnation point measurements under different chemical regimes. The design of these probes was presented by Herpin [103] when studying the scaling of the chemistry in non-equilibrium flows. Dimensions of each probe are specified in Table 4.1. The three probes are water-cooled and made of copper. They feature a central bore to house the calorimeter shown in Fig. 3.5. The ESA-Standard probe extensively used in Sec. 3.1. from which catalysis is already know $\left(\gamma_{r e f}\right)$ and used to rebuild the $H_{e}$ conditions with CERBOULA, is used as reference. The other two probes either promote or hamper the gas-phase recombination when compared to the reference configuration. The concept behind the design of these three probes consists on changing $D a_{g}$ between them while keeping the same plasma free-stream conditions. Indeed, increasing the size of the probe will lead to a thicker boundary layer, making the diffusion zone larger and providing the gas more time to recombine. Thus, the larger holder is known as the Equilibrium probe because it increases $D a_{g}$ when compared to the Standard probe. Conversely, the smaller probe is named Frozen probe because it tends to decrease the $D a_{g}$.

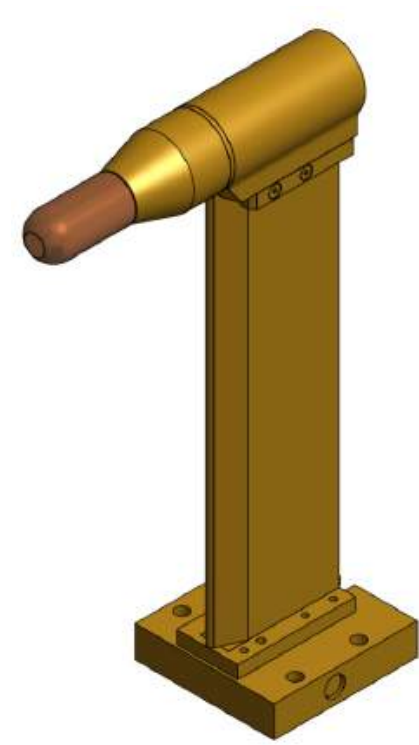

(a) Frozen (FR)

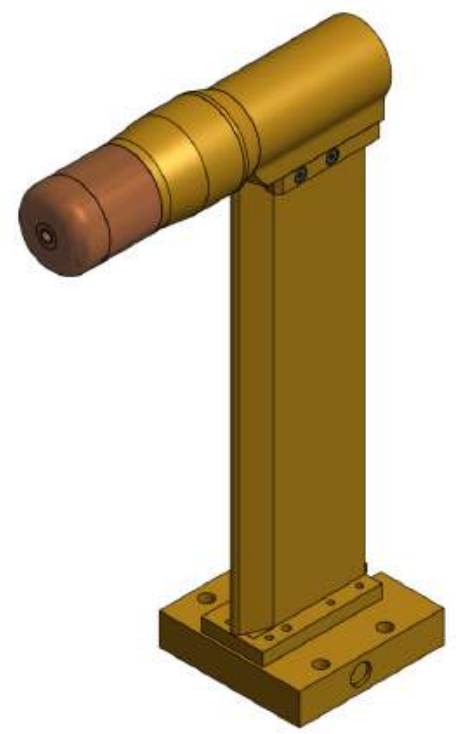

(b) Standard (ST)

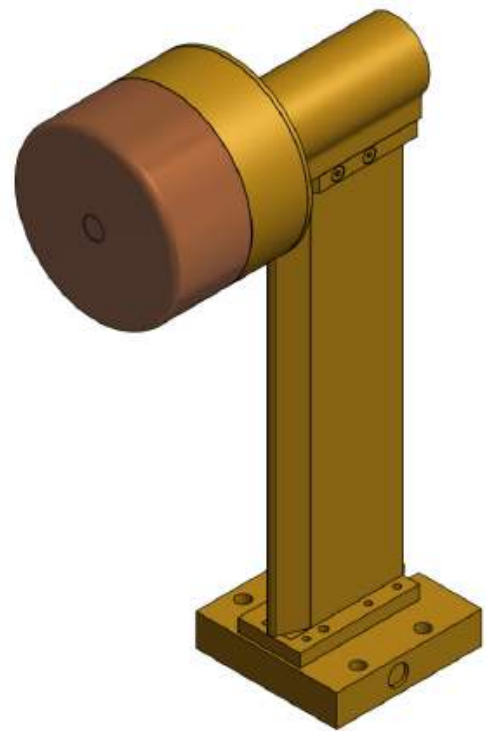

(c) Equilibrium (EQ)

Figure 4.6.: The VKI Damköhler probes. 
Table 4.1.: Dimensions of the Damköhler probes

\begin{tabular}{lccc}
\hline \hline Probe & Symbol & $\begin{array}{c}R_{b} \\
\mathrm{~mm}\end{array}$ & $\begin{array}{c}R_{c} \\
\mathrm{~mm}\end{array}$ \\
\hline Standard & $\mathrm{ST}$ & 25 & 11.75 \\
Equilibrium & $\mathrm{EQ}$ & 57.5 & 5 \\
Frozen & $\mathrm{FR}$ & 15 & 15 \\
\hline \hline
\end{tabular}

The Damköhler probes were already used by Krassilchikoff in the so-called "Equilibrium flow extrapolation" [91 as an alternative to the Mini-Max for $\gamma_{r e f}$ definition. With that testing campaign not fully successful, de Crombrugghe measured catalysis on both the Frozen and the Equilibrium probes to investigate qualitatively the behavior of GSI phenomena under different gas-phase Damköhler numbers in the context of the LHTS methodology [95]. Additional tests were also carried out by Panerai [11] on $\mathrm{C} / \mathrm{SiC}$ samples using both the Standard and the Equilibrium probes. He showed how different probe geometries led to different values of $\gamma$ on the same material. However, since the surface temperature on $\mathrm{C} / \mathrm{SiC}$ was not the same among the different probes, the non-equilibrium effect on the determination of $\gamma$ could not be properly assessed. In fact, the main difference with the campaign presented below is the control of $T_{w}$ on the calorimeters. While TPS material surface temperatures can change by several hundreds of degrees from one probe to another, it is reasonable to assume that the surface of the water-cooled calorimeter remains at $350 \mathrm{~K}$ for any probe configuration.

In order to illustrate the influence of the probe design on the boundary layer chemical nonequilibrium properties, examples of both atomic oxygen and nitrogen mass fractions profiles computed with CERBOULA for the three Damköhler probes are shown in Fig. 4.7. They are obtained under the same plasma conditions of temperature and pressure, and the non-catalytic assumption is imposed so that species profiles are consequence of gas-phase recombination only. As expected, the Frozen probe leads to a thinner chemical boundary layer when compared to both the Standard and the Equilibrium probes. The effect of the probe geometry on the amount of gas-phase recombination is clear if one observes the N profiles among the three probes. Indeed, the mass fraction at the wall of the Equilibrium probe is significantly lower than the value measured for the Frozen probe, with the mass fraction at the wall of the Standard probe falling in between. On the other hand, the O-atom profile remains almost frozen and fully dissociated for the three probes. Fig. 4.7 also demonstrates that non-equilibrium boundary layers are present with any probe configuration, meaning that the size of the probe favors either frozen or equilibrium regimes, but it cannot enforce the development of purely frozen $\left(D a_{g} \rightarrow 0\right)$ or purely equilibrium $\left(D a_{g} \rightarrow \infty\right)$ boundary layers.

\subsubsection{Off-stagnation point: the flat plate}

Tests at off-stagnation point are also possible in the Plasmatron facility. The strategy is to use a flat plate holder to test rectangular samples in a shear flow configuration. The flat plate holder available in VKI was designed by Vanaerschot [104] and it is shown in Fig. 4.8. Following the same logic of the stagnation point holders, he proposed a water-cooled copper solution. Although the 


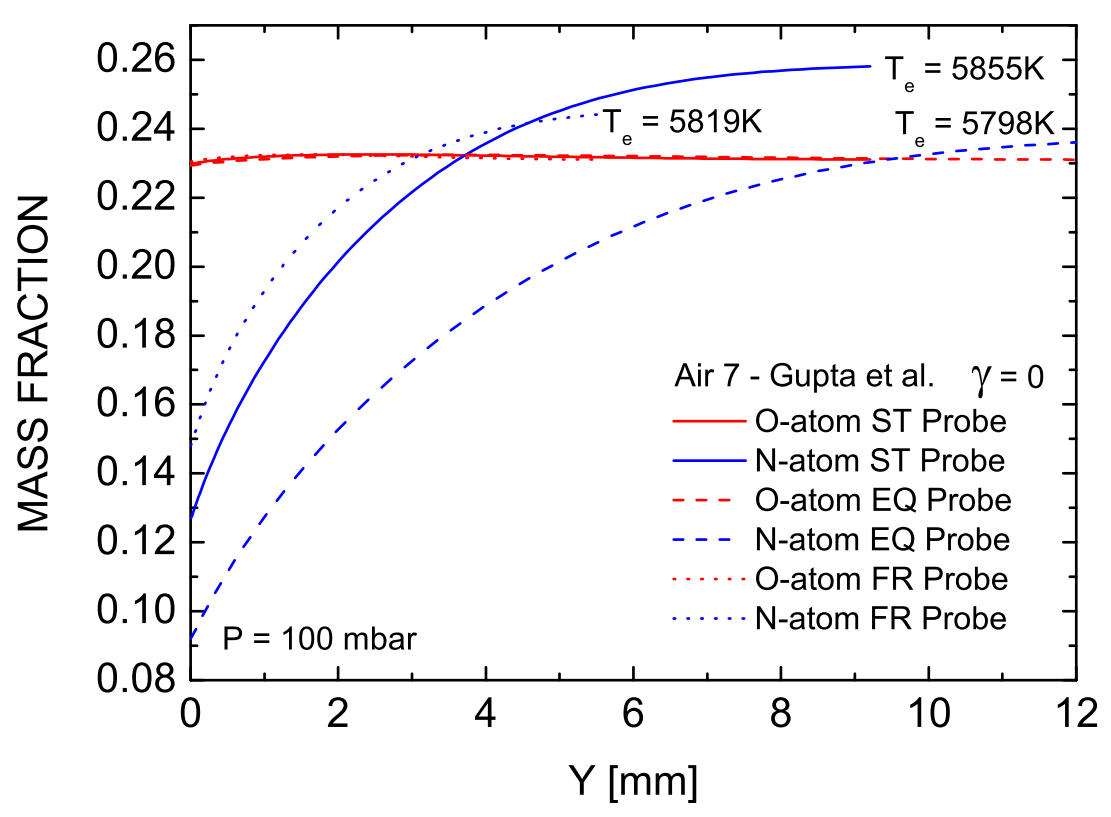

Figure 4.7.: Example of the $\mathrm{O}$ and $\mathrm{N}$ profiles among the Damköhler probes for the same testing conditions of temperature and pressure and a non-catalytic wall.

ideal design would be a flat plate as thin as possible, the necessity to cool the system led to a holder that is $25 \mathrm{~mm}$ thick. It measures $240 \mathrm{~mm}$ long by $200 \mathrm{~mm}$ wide and the leading edge is rounded with corners of $12.5 \mathrm{~mm}$ radius. The holder has a groove to locate the rectangular samples for testing. This groove starts $51 \mathrm{~mm}$ from the leading edge and is $189 \mathrm{~mm}$ long by $30 \mathrm{~mm}$ wide.

A new calorimeter concept is especially designed and manufactured in the VKI to be inserted in the flat plate holder. This is the linear calorimeter shown in Fig. 4.9. As the stagnation point calorimeter, it is made of copper and the heat flux is determined by measuring the energy exchange with the water circulating inside the system. It contains 8 heat flux sensors, and the temperature of the water is measured at the inlet and outlet of each sensing element, as seen in Fig. 4.10, using a total of 9 E-thermocouples. The insulation of the calorimeter is obtained with PEEK, which offers good performance at high temperatures and it is not too brittle [104]. The piece of PEEK insulates the calorimeter from the holder, while any energy exchange between two consecutive heat flux sensors is prevented by the isothermal wall condition along the linear calorimeter, assumed at $350 \mathrm{~K}$. Such hypothesis is reasonable because the surface temperature evolution along the flat plate is expected to be much smaller than the temperature change across the boundary layer. For installation, the calorimeter slides in the flat plate holder. 


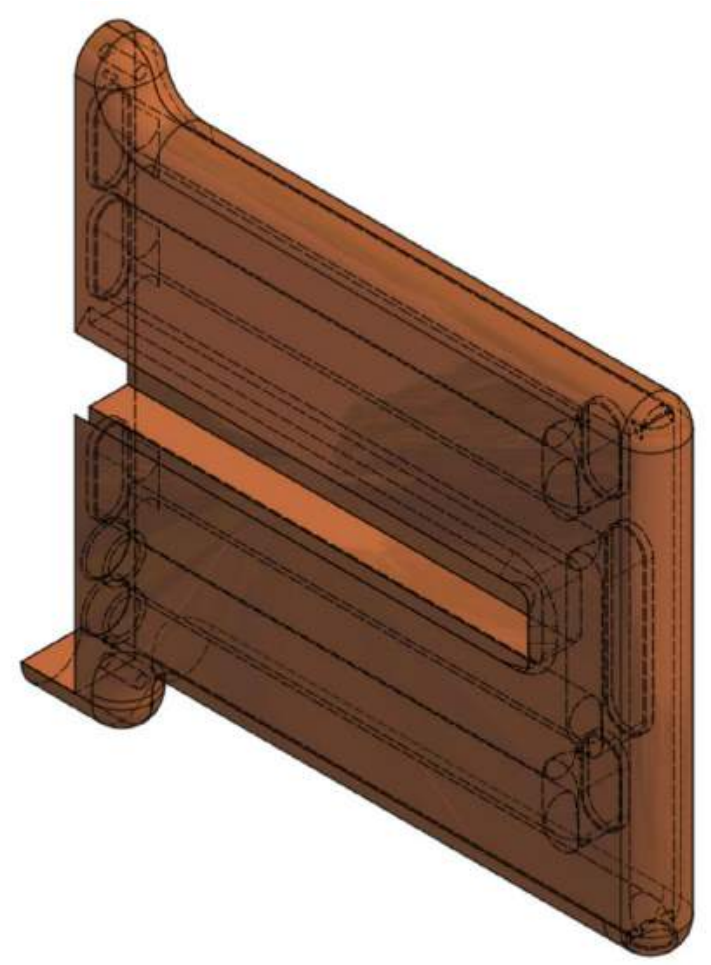

Figure 4.8.: Flat plate holder. Courtesy: Vanaerschot [104].

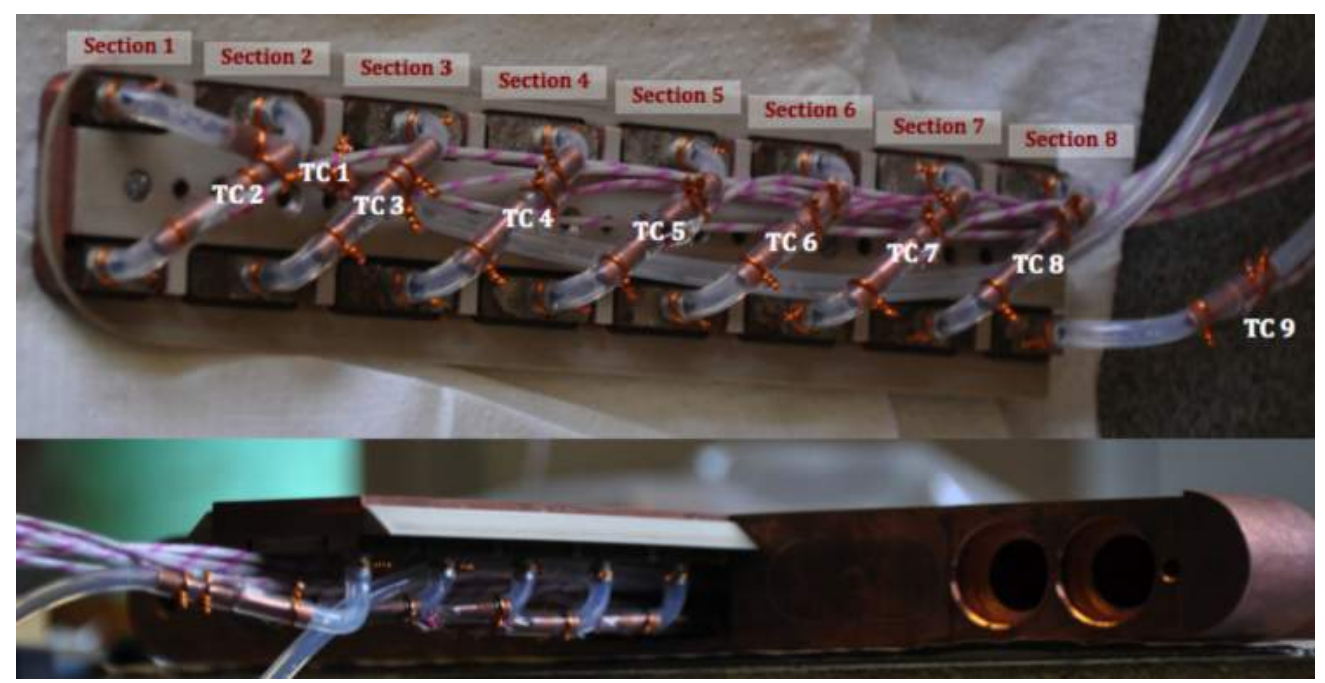

Figure 4.9.: Linear calorimeter. Courtesy: Vanaerschot [104].

The flat plate system is placed vertically in the Plasmatron test chamber, as seen in Fig. 4.11. This configuration has already been tested [105, 106] for the validation of a boundary layer extrapolation methodology at off-stagnation point. Tests show that the operating envelope for this configuration is smaller compared to the stagnant configuration. This is due to the high temperature reached at the last sensor of the calorimeter. Plasmatron power settings cannot be too high in order to prevent the circulating water inside the pipe from boiling. 


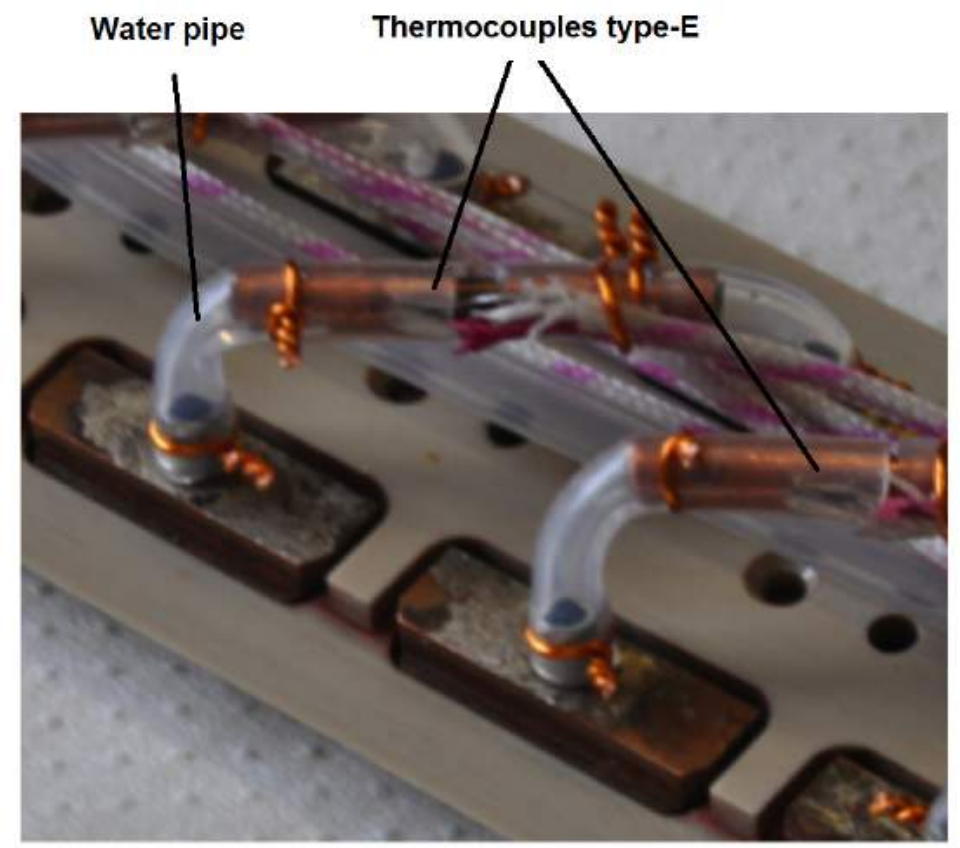

Figure 4.10.: Details of a heat flux sensor in the linear calorimeter. Courtesy: Vanaerschot [104].

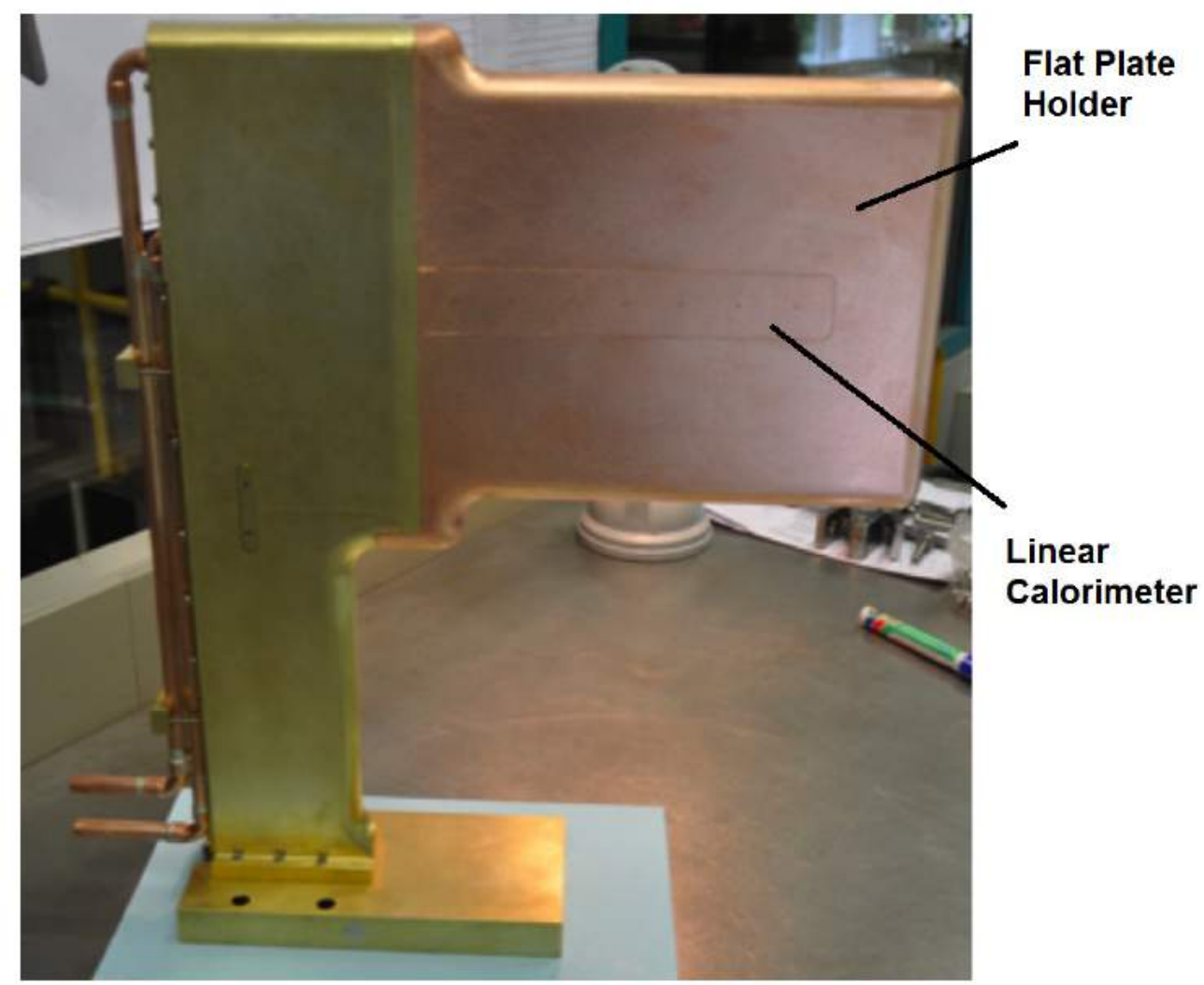

Figure 4.11.: The flat plate holder with the linear calorimeter installed. Courtesy: Vanaerschot [104]. 


\subsection{Catalysis determination at stagnation point}

A test campaign is carried out with the Plasmatron to address the possible relation between the chemical non-equilibrium characteristics of the boundary layer and the wall catalysis determined with CERBOULA. To this end, the three Damköhler probes are exposed to the same plasma conditions of enthalpy $H_{e}$, mass flow of air $\dot{m}$ and static pressure $P_{s}$, as shown in Fig. 4.12. The probes are installed $445 \mathrm{~mm}$ downstream from the torch exit and aligned with the center of the jet. The Standard and the Equilibrium holders are installed on the lower injection system, while the Frozen holder is installed on the upper one, at the top of the chamber.

A copper calorimeter is inserted at the center of each probe, the mass flow of water that is circulating inside each calorimeter is kept constant using rotameters, and its temperature difference between the inlet and the outlet is measured with pairs of E-thermocouples. The computation of the heat flux is carried out in real time by the data acquisition system and recorded at $1 \mathrm{~Hz}$.

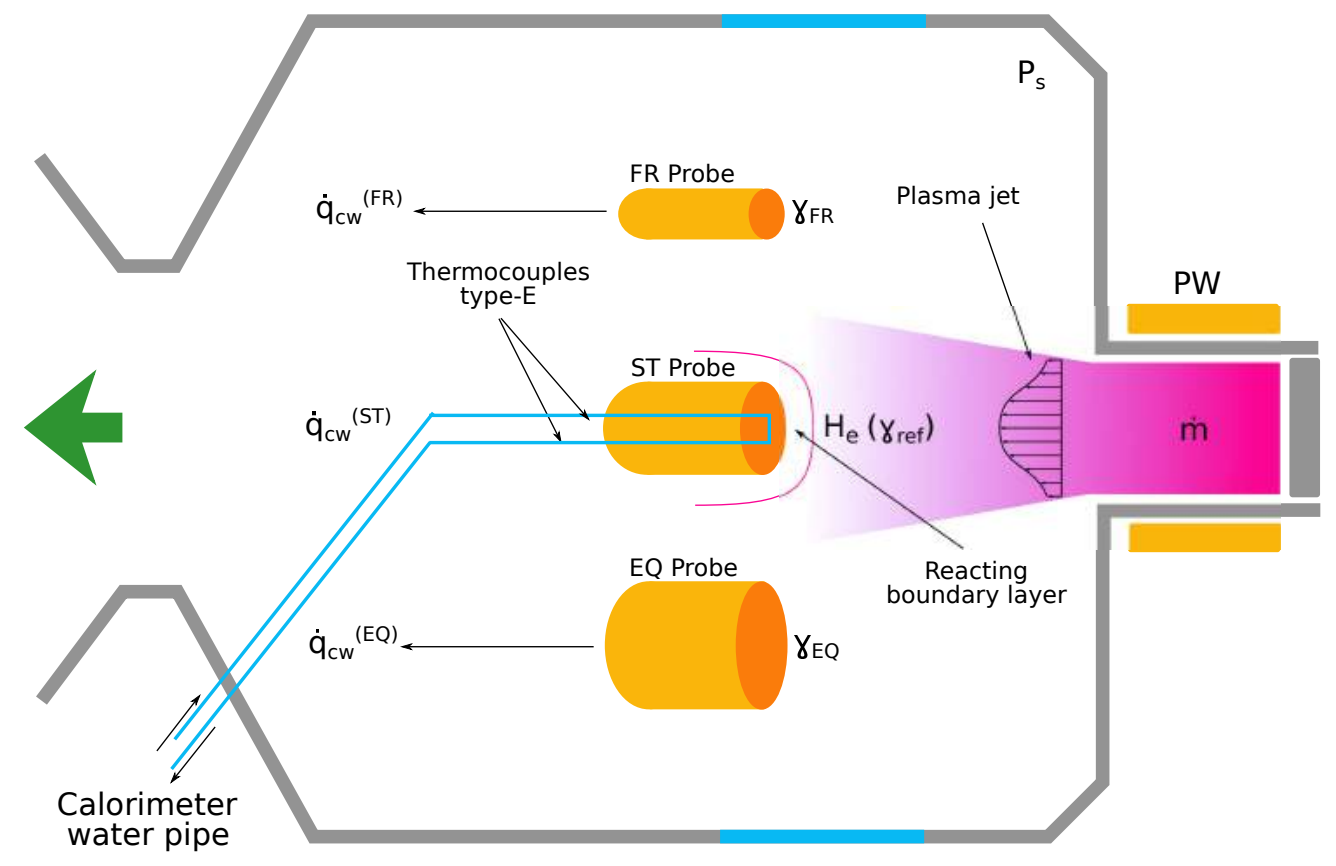

Figure 4.12.: Plasmatron test configuration for catalysis determination on the Damköhler probes.

The same testing conditions from 1a to 9c that were used in Sec. 3.2 are applied again here. Remember that letters a, b and c refer to static pressures of 15, 50 and 100 mbar, respectively. Note that tests at 200 mbar are not being considered during this campaign due to the low influence of catalysis under such high pressure conditions, as reported by the Mini-Max method. The mass flow rate of air entering the chamber is set constant at $16 \mathrm{~g} / \mathrm{s}$, and the power applied to the coil $P W$ is varied according to the target heat flux conditions on the Standard probe, which is used as reference to define $H_{e}$ during the post processing with CERBOULA. Heat flux measurements on the three probes are listed in Table 4.2 , together with the target condition $\dot{q}_{c w}^{(r e f)}$ and $P W$.

Due to the fact that only three probes can be installed in the Plasmatron chamber at the same time, the dynamic pressure measurements can not be taken. Instead, the same interpolated values that were measured during the Mini-Max testing campaign are used to post-process the data on the Damköhler probes. Such procedure is reasonable because the target testing conditions are the 
Table 4.2.: Heat flux measurements during the Damköhler probes test campaign: a) 15 mbar; b) 50 mbar; c)100 mbar

\begin{tabular}{crrrrr}
\hline \hline Test & $\begin{array}{r}\dot{q}_{c w}^{(r e f)} \\
\mathrm{kW} \mathrm{m}^{-2}\end{array}$ & $\begin{array}{r}P W \\
\mathrm{~kW}\end{array}$ & $\begin{array}{r}\dot{q}_{c w}^{(S T)} \\
\mathrm{kW} \mathrm{m}^{-2}\end{array}$ & $\begin{array}{r}\dot{q}_{c w}^{(F R)} \\
\mathrm{kW} \mathrm{m}^{-2}\end{array}$ & $\begin{array}{r}\dot{q}_{c w}^{(E Q)} \\
\mathrm{kW} \mathrm{m}^{-2}\end{array}$ \\
\hline 1a & 300 & 92 & 309.90 & 321.67 & 132.28 \\
2a & 500 & 126 & 521.20 & 492.30 & 172.48 \\
3a & 700 & 153 & 704.53 & 734.24 & 325.58 \\
4a & 900 & 183 & 898.90 & 971.20 & 469.84 \\
5a & 1100 & 207 & 1102.27 & 1189.48 & 584.99 \\
6a & 1300 & 230 & 1282.67 & 1379.59 & 742.89 \\
7a & 1500 & 255 & 1483.82 & 1570.31 & 874.83 \\
8a & 1700 & 288 & 1697.52 & 1822.02 & 1075.31 \\
9a & 2000 & 335 & 2044.36 & 2098.13 & 1335.84 \\
\hline 1b & 300 & 125 & 297.10 & 401.79 & 74.84 \\
2b & 500 & 148 & 464.09 & 541.82 & 107.97 \\
3b & 700 & 168 & 666.64 & 789.66 & 231.35 \\
4b & 900 & 187 & 862.94 & 1047.18 & 380.45 \\
5b & 1100 & 244 & 1070.71 & 1306.58 & 459.41 \\
6b & 1300 & 252 & 1327.23 & 1492.22 & 665.90 \\
7b & 1500 & 278 & 1496.27 & 1750.11 & 841.29 \\
8b & 1700 & 297 & 1708.32 & 2083.47 & 1048.70 \\
9b & 2000 & 323 & 2018.90 & 2315.14 & 1214.37 \\
\hline 1c & 300 & 136 & 276.64 & 344.27 & 104.35 \\
2c & 500 & 145 & 524.24 & 629.82 & 254.24 \\
3c & 700 & 187 & 673.32 & 1059.45 & 419.42 \\
4c & 900 & 192 & 926.48 & 1100.61 & 565.62 \\
5c & 1100 & 225 & 1099.08 & 1360.03 & 594.82 \\
6c & 1300 & 266 & 1286.49 & 1773.63 & 807.58 \\
7c & 1500 & 276 & 1490.70 & 1826.35 & 886.47 \\
8c & 1700 & 300 & 1678.81 & 2059.42 & 1050.43 \\
9c & 2000 & 330 & 1967.04 & 2377.24 & 1279.21 \\
\hline \hline & & & & & \\
\hline
\end{tabular}


same in both experiments. With the purpose of matching consistently the heat flux measurements and the dynamic pressure data with the same target condition, a linear regression of the form $\dot{q}_{c w}^{(i)}=y_{0}+m \dot{q}_{c w}^{(r e f)}$ is carried out on all the measured quantities using the heat flux on the Standard probe as reference. The resulting coefficients of such approximation are provided in Table 4.3 , together with the coefficient of determination $\mathrm{R}^{2}$ of the fit.

Table 4.3.: Linear interpolation coefficients for the heat fluxes measured on the Damköhler probes $\left(\dot{q}_{c w}^{(i)}=y_{0}+m \dot{q}_{c w}^{(r e f)}\right)$

\begin{tabular}{lcrrr}
\hline \hline$P_{s}$ & Probe (i) & $y_{0}$ & $m$ & $\mathrm{R}^{2}$ \\
\hline $15 \mathrm{mbar}$ & $\mathrm{FR}$ & -4.9773 & 1.0576 & 0.9966 \\
$15 \mathrm{mbar}$ & $\mathrm{EQ}$ & -167.48 & 0.7189 & 0.9916 \\
$50 \mathrm{mbar}$ & $\mathrm{FR}$ & 40.1462 & 1.1467 & 0.9953 \\
$50 \mathrm{mbar}$ & $\mathrm{EQ}$ & -211.53 & 0.6990 & 0.9851 \\
$100 \mathrm{mbar}$ & $\mathrm{FR}$ & 77.888 & 1.1922 & 0.9784 \\
$100 \mathrm{mbar}$ & $\mathrm{EQ}$ & -83.697 & 0.6768 & 0.9911 \\
\hline \hline
\end{tabular}

Nine Plasmatron power levels, at three different pressure conditions and under three different geometries lead to a total of 81 ICP simulations that are carried out in CoolFluid to estimate the NDP's. They are listed in Tables B.2 to B.4. A power efficiency of $50 \%$ is assumed to run the computations. Then, the reference heat flux measurement, combined with $\gamma_{\text {ref }}$, provides the outer edge enthalpy for the 27 testing conditions listed in Table 4.2 using CERBOULA. Computing also the $H_{e^{-}} \gamma$ relation for both the Frozen and the Equilibrium probes, and using $H_{e}$, leads to the determination of the recombination coefficients $\gamma_{F R}$ and $\gamma_{E Q}$. Note that, as in the Mini-Max test campaign, the chemical model proposed by Gupta et al. [2] is used for post-processing. Results are shown and discussed below for each pressure condition.

\subsubsection{Analysis of the Damköhler probes at $15 \mathrm{mbar}$}

The post-processing for catalysis determination is difficult at 15 mbar, mostly because the convergence of the iterative process carried out by CERBOULA lacks robustness due to the high sensitivity of $H_{e}$ with respect to $\gamma$. Such sensitivity is due to the lack of collisions between particles at low pressures, requiring a very high $H_{e}$ to match certain experimental heat flux values at lower catalytic conditions (see Fig. 4.1).

This effect can be observed in Table 4.4, containing the outer edge enthalpy, the recombination coefficients and the boundary layer thicknesses on the three Damköhler probes at 15 mbar. Particularly, the recombination coefficients on the Equilibrium probe can not be determined because CERBOULA is not able to compute the $H_{e^{-}} \gamma$ relation in this configuration. It would seem that the combination of a low pressure environment and a probe geometry that induces volume reactions leads to a poorly-defined condition for CERBOULA that converges to an unphysical solution. Remark also that there are situations where the NDP's computations are not correct for the Equilibrium probe. For instance, the $\delta_{E Q}$ values provided by ICP simulations for conditions 1a, 8a and 9a are smaller than the $\delta_{S T}$ computations at the same test condition. There is no explanation of 
this anomaly yet, and the effort required to elucidate this issue is beyond the scope of this dissertation. In the author's opinion, a deeper investigation on the combination of ICP computations in CoolFluid and CERBOULA should be carried out in the near future.

Table 4.4.: Outer edge enthalpy, recombination coefficients and boundary layer thicknesses at 15 mbar on the Damköhler probes

\begin{tabular}{lrrrrrrr}
\hline \hline Test & $\begin{array}{r}H_{e} \\
\mathrm{MJ} \mathrm{kg}^{-1}\end{array}$ & $\gamma_{r e f}$ & $\begin{array}{r}\delta_{S T} \\
\mathrm{~mm}\end{array}$ & $\gamma_{F R}$ & $\begin{array}{r}\delta_{F R} \\
\mathrm{~mm}\end{array}$ & $\gamma_{E Q}$ & $\begin{array}{r}\delta_{E Q} \\
\mathrm{~mm}\end{array}$ \\
\hline $1 \mathrm{a}$ & 7.59 & 0.07738 & 11.27 & 0.02404 & 4.25 & - & 1.17 \\
$2 \mathrm{a}$ & 11.46 & 0.07738 & 11.20 & 0.02339 & 4.29 & - & 36.13 \\
3a & 15.26 & 0.07738 & 11.07 & 0.03163 & 4.19 & - & 31.54 \\
$4 \mathrm{a}$ & 19.11 & 0.07738 & 10.83 & 0.03702 & 4.15 & - & 29.61 \\
$5 \mathrm{a}$ & 23.09 & 0.07738 & 10.71 & 0.04638 & 4.13 & - & 34.01 \\
$6 \mathrm{a}$ & 27.21 & 0.07738 & 10.80 & 0.04557 & 4.14 & - & 46.28 \\
$7 \mathrm{a}$ & 31.41 & 0.07738 & 11.85 & 0.05151 & 4.04 & - & 48.54 \\
$8 \mathrm{a}$ & 35.58 & 0.07738 & 11.48 & 0.05854 & 4.69 & - & 9.38 \\
$9 \mathrm{a}$ & 40.67 & 0.07738 & 10.76 & 0.05664 & 4.93 & - & 6.67 \\
\hline \hline
\end{tabular}

The recombination coefficients obtained on the Frozen probe $\gamma_{F R}$ are shown and compared to $\gamma_{r e f}$ in Fig. 4.13. Error bars around $\gamma_{F R}$ are introduced assuming an uncertainty of $\pm 13 \%$ on $H_{e}$ for the Standard probe [94]. One can observe that the recombination coefficient measured on the smaller probe is lower than $\gamma_{r e f}$ for all the test conditions. This is an unexpected result considering the fact that there is less volume recombination taking place in the Frozen probe, as previously seen in Fig. 4.7. One possible explanation is that both $N u$ and $D a_{g}$ decrease when changing from Standard to Frozen probe configurations and, by consistency with the discussion of Sec. 4.1, this leads to lower values of $D a_{w}$ and, in consequence, to smaller recombination coefficients. Remark that these results demonstrate that the non-equilibrium properties of the boundary layer can affect the experimentally determined recombination coefficient.

\subsubsection{Analysis of the Damköhler probes at $50 \mathrm{mbar}$}

The post-processing at 50 mbar is easier than at 15 mbar. Results for $H_{e}, \gamma$ and $\delta$ are listed in Table 4.5 for the three probes. Note that, although the recombination coefficients on the Equilibrium probe could be determined in most of the situations, CERBOULA did not converge at both the lowest and the highest powers of the campaign. Remark also that, contrary to the test at 15 mbar, boundary layer thicknesses behavior is consistent for the three probes.

The experimentally determined recombination coefficients on the Damköhler probes at 50 mbar are shown in Fig. 4.14 together with $\gamma_{r e f}$. Again, error bars are computed assuming $\pm 13 \%$ uncertainty for $H_{e}$. It is observed that both $\gamma_{E Q}$ and $\gamma_{F R}$ are lower than $\gamma_{r e f}$, with $\gamma_{E Q}$ being the smallest. The recombination coefficient for the Equilibrium probe shows a marked growth for increasing values of $H_{e}$. Note that none of the $\gamma_{E Q}$ error bars cross their $\gamma_{r e f}$ counterparts. Regarding $\gamma_{F R}$, it shows the same trend as in the 15 mbar test. However, it seems that the Frozen and the Standard probes have similar catalytic activities. It is worth reminding that this influence of $H_{e}$ 
(or power) on $\gamma$ was not observed in the Mini-Max testing campaign.

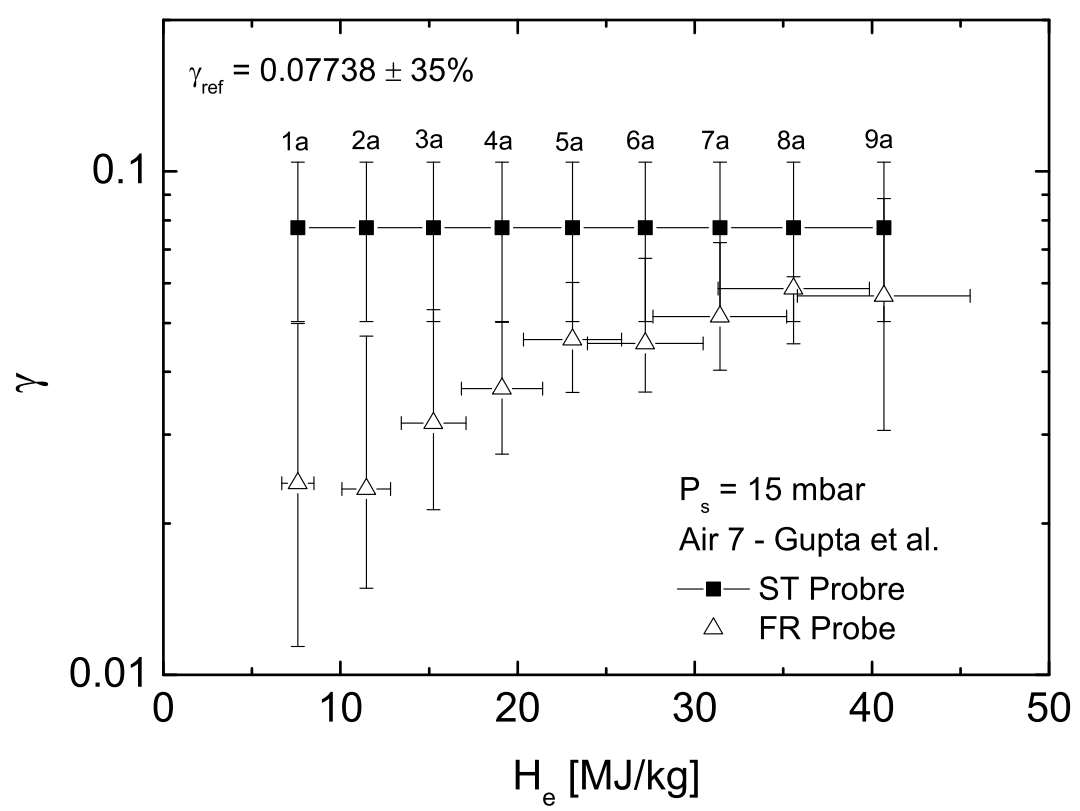

Figure 4.13.: Recombination coefficients on the Damköhler probes at 15 mbar.

Table 4.5.: Outer edge enthalpy, recombination coefficients and boundary layer thicknesses at 50 mbar on the Damköhler probes

\begin{tabular}{lrrrrrrr}
\hline \hline Test & $\begin{array}{r}H_{e} \\
\mathrm{MJ} \mathrm{kg}^{-1}\end{array}$ & $\gamma_{\text {ref }}$ & $\begin{array}{r}\delta_{S T} \\
\mathrm{~mm}\end{array}$ & $\gamma_{F R}$ & $\begin{array}{r}\delta_{F R} \\
\mathrm{~mm}\end{array}$ & $\gamma_{E Q}$ & $\begin{array}{r}\delta_{E Q} \\
\mathrm{~mm}\end{array}$ \\
\hline 1b & 9.88 & 0.02661 & 11.09 & 0.00948 & 4.27 & - & 27.39 \\
2b & 13.41 & 0.02661 & 10.92 & 0.00885 & 4.11 & - & 23.88 \\
3b & 16.88 & 0.02661 & 10.64 & 0.01039 & 4.04 & 0.00212 & 20.94 \\
4b & 20.31 & 0.02661 & 10.41 & 0.01165 & 3.98 & 0.00490 & 19.63 \\
5b & 23.78 & 0.02661 & 10.29 & 0.01599 & 3.96 & 0.00818 & 20.28 \\
6b & 27.48 & 0.02661 & 10.32 & 0.01833 & 3.94 & 0.00919 & 26.18 \\
7b & 32.03 & 0.02661 & 10.67 & 0.01834 & 3.87 & 0.00874 & 30.94 \\
8b & 36.49 & 0.02661 & 11.17 & 0.01801 & 3.92 & 0.01024 & 29.27 \\
9b & 41.36 & 0.02661 & 10.79 & 0.01739 & 4.55 & - & 20.80 \\
\hline \hline
\end{tabular}




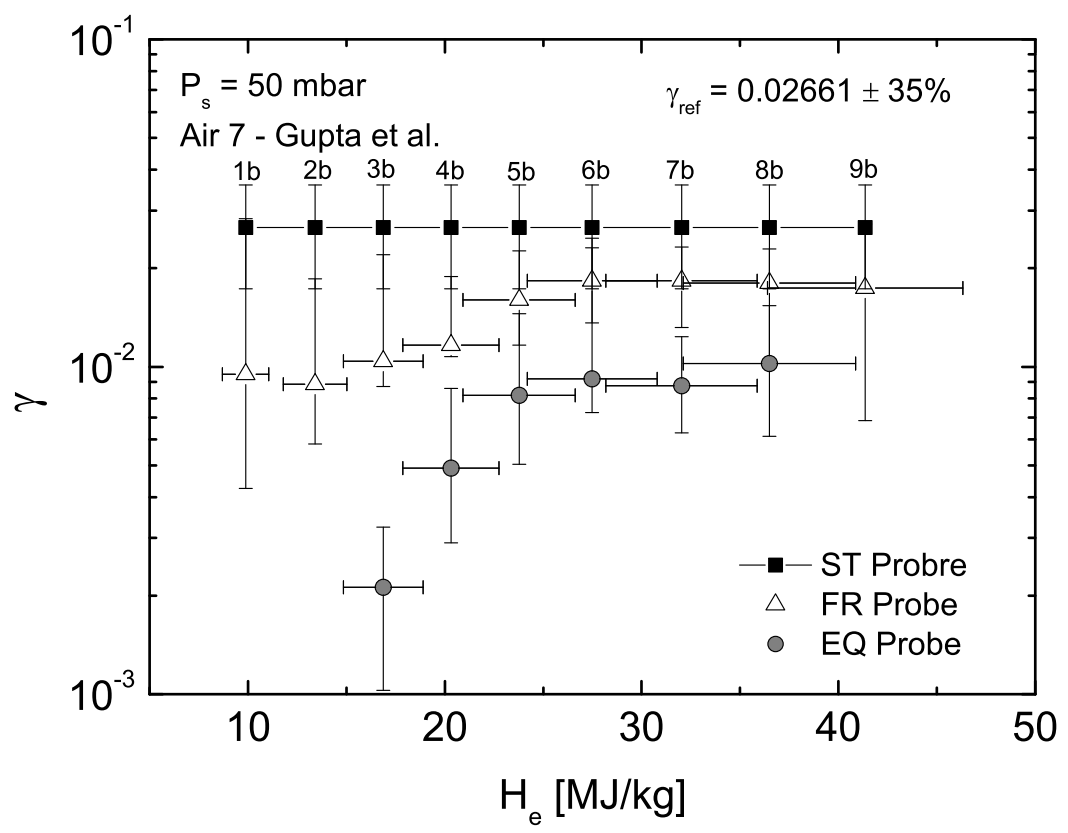

Figure 4.14.: Recombination coefficients on the Damköhler probes at 50 mbar.

Given the size of each probe, one could expect that values of $\gamma_{r e f}$ would be in between $\gamma_{E Q}$ and $\gamma_{F R}$. However, Fig. 4.14 shows that this is not the case. The computation of the non-dimensional numbers that intervene in the non-equilibrium characterization of the boundary layer can bring some understanding for these non-anticipated results. These parameters are $D a_{g}, D a_{w}, N u$ and $H$, as explained in Sec. 4.1. The definition of $H$ is:

$$
H=L e^{-0.6} \frac{\Delta h_{\text {chem }, e q}}{\Delta h_{f r}}
$$

for a boundary layer developed in a stagnant flow, where $L e$ is the Lewis number $\left(L e=\lambda / \rho D c_{p}\right)$ and it plays the role of a recovery factor for the chemical energy exchange [99]. From Eq. 4.14, one could see that $H$ changes with power, but not among the probes under the same testing conditions. This means that, for each Plasmatron test condition (from 1a to 9c), one could draw the same surface $N u=f\left(D a_{g}, D a_{w}\right)$, shown in Fig. 4.3, and from which the $N u$ map plotted in Fig. 4.15 is obtained.

For the analysis, the $D a_{g}$ value obtained at 50 mbar is shown in Fig. 4.16, It is computed with:

$$
D a_{g}=\frac{[\mathrm{N}] \delta^{2}}{D}\left(4 k_{f}[\mathrm{~N}]_{E q}-k_{b}\right)
$$

which takes into account the nitrogen recombination $2 \mathrm{~N}+\mathrm{M} \longrightarrow \mathrm{N}_{2}+\mathrm{M}$ as reference reaction to estimate the characteristic time of the chemistry, with atomic nitrogen acting as collision partner M. As expected, $D a_{g}$ on the Standard probe falls between the values determined for the other two probes, and they all increase with power (or equivalently, the amount of atomic species at the outer edge). 


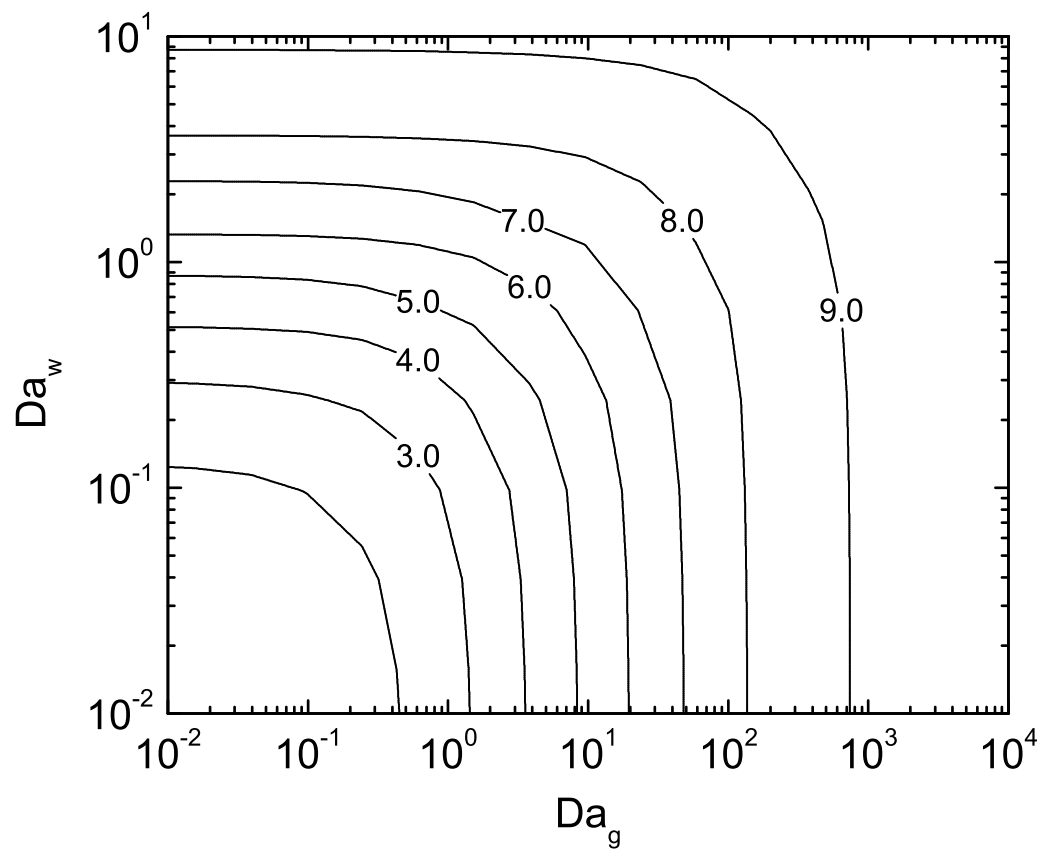

Figure 4.15.: Two-dimensional map of the $N u$ number as a function of $D a_{g}$ and $D a_{w}$ for $H=9$, as proposed by Rosner [99].

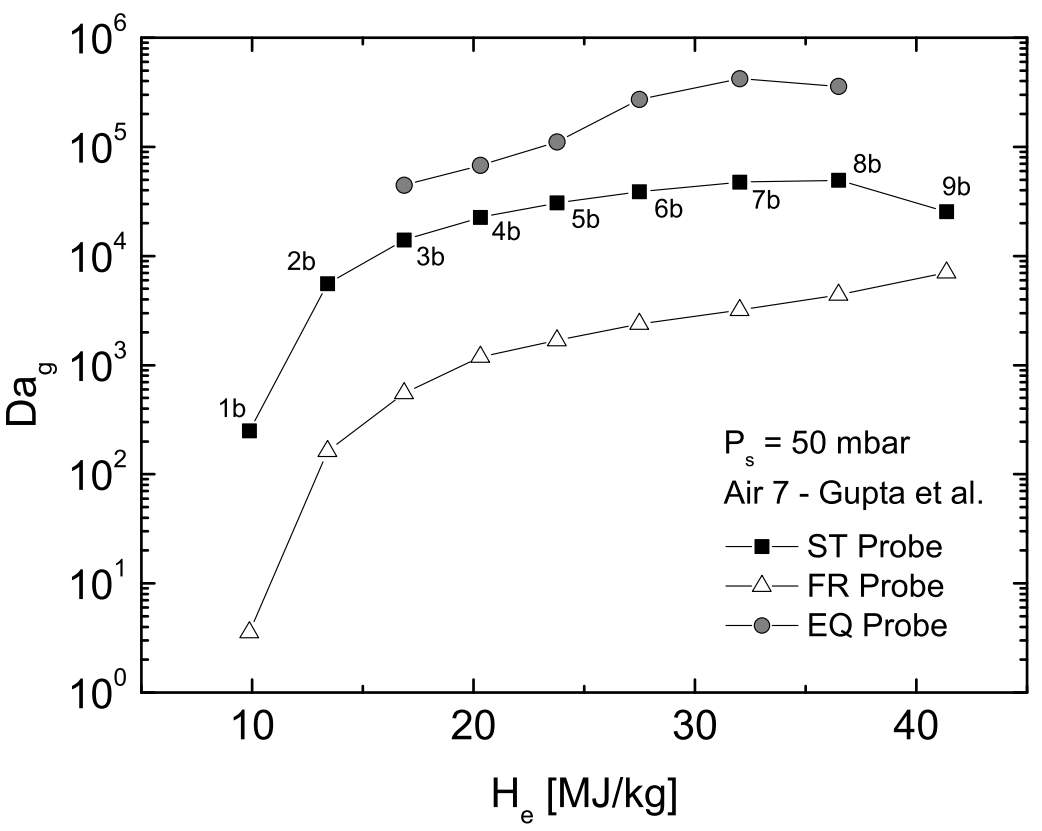

Figure 4.16.: $D a_{g}$ on the Damköhler probes at 50 mbar.

$D a_{w}$ and $N u$ are shown in Fig. 4.17a and Fig. 4.17b, respectively. The combination of $\gamma_{F R}<\gamma_{r e f}$ and $\delta_{F R}<\delta_{S T}$ brings $D a_{w}$ on the Frozen probe to the bottom of the plot. As both Damköhler numbers are lower for the Frozen probe than for the Standard one, the Nusselt number of the former is also smaller. This is consistent with the surface $N u=f\left(D a_{g}, D a_{w}\right)$ shown in Fig. 4.3. 


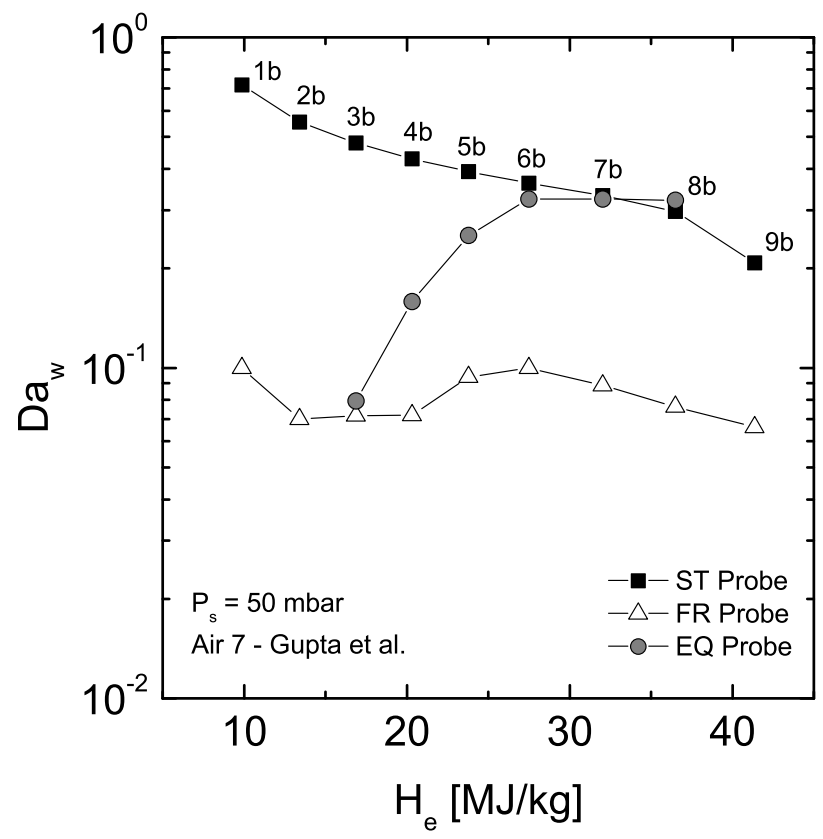

(a) $D a_{w}$ on the Damköhler probes.

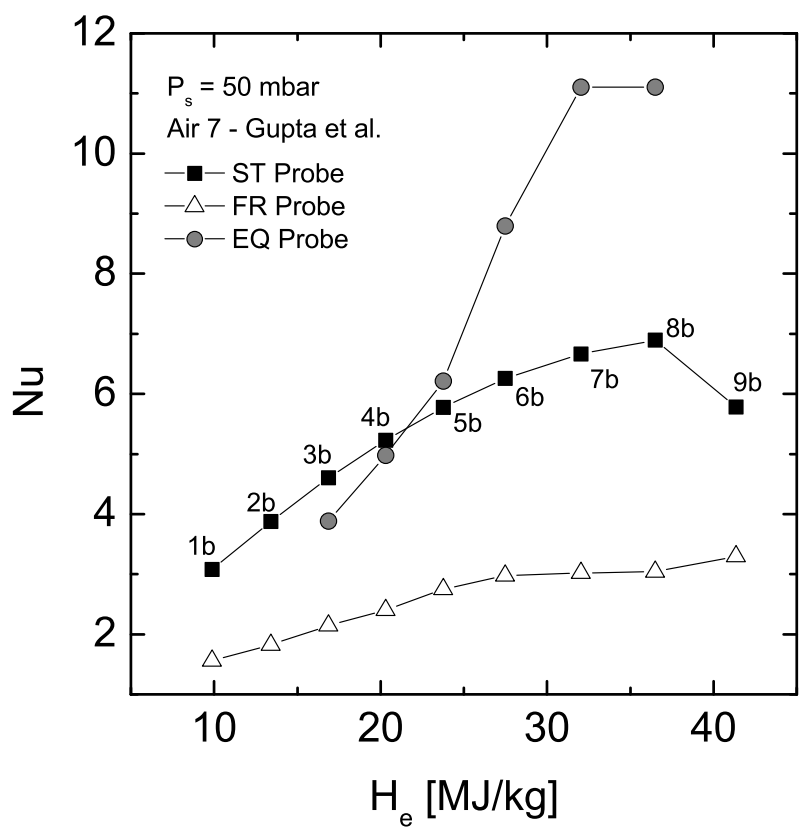

(b) $N u$ on the Damköhler probes.

Figure 4.17.: Non-dimensional numbers obtained at 50 mbar.

Considering the Equilibrium probe, it is observed that $D a_{w}^{(E Q)}<D a_{w}^{(S T)}$ from tests $3 \mathrm{~b}$ to $5 \mathrm{~b}$ while $N u^{(E Q)}=N u^{(S T)}$. The relevance of this result lies on the fact that it validates the work of Rosner (see Sec. 4.1). Indeed, increasing $D a_{g}$ when switching from the Standard to the Equilibrium probe while keeping the same $N u$, reduces $D a_{w}$ and, eventually, leads to a recombination coefficient reduction. Then, it would seem that an increase of gas recombination induced by the Equilibrium probe can be compensated by a reduction of surface reactions thus maintaining the Nusselt number.

It is also observed that $D a_{w}^{(E Q)}=D a_{w}^{(S T)}$ from conditions $6 \mathrm{~b}$ to $8 \mathrm{~b}$ and $N u^{(E Q)}>N u^{(S T)}$. This is also consistent with Fig. 4.15 because an increase in $D a_{g}$ leads to an increase of $N u$ if $D a_{w}$ is kept the same, which results in similar recombination coefficients for the Equilibrium and Standard probes.

From these results, one could conclude that the reduction of the recombination coefficient on both Frozen and Equilibrium probes is consequence of the coupling between $N u, D a_{g}$ and $D a_{w}$ for constant $H$. This demonstrates the influence of the non-equilibrium environment when determining experimentally the catalytic properties of surfaces.

\subsubsection{Analysis of the Damköhler probes at $100 \mathrm{mbar}$}

The results at 100 mbar are similar to those at 50 mbar and they are provided in Table 4.6. In this case, CERBOULA did not converge for tests $1 \mathrm{c}$ and $9 \mathrm{c}$ on the Equilibrium probe. The recombination coefficients are shown in Fig. 4.18 assuming $\pm 13 \%$ uncertainty for $H_{e}$ and, again, the values on both the Frozen and the Equilibrium probes are lower than $\gamma_{r e f}$. 
Table 4.6.: Outer edge enthalpy, recombination coefficients and boundary layer thicknesses at 100 mbar on the Damköhler probes

\begin{tabular}{lrrrrrrr}
\hline \hline Test & $\begin{array}{r}H_{e} \\
\mathrm{MJ} \mathrm{kg}^{-1}\end{array}$ & $\gamma_{\text {ref }}$ & $\begin{array}{r}\delta_{S T} \\
\mathrm{~mm}\end{array}$ & $\gamma_{F R}$ & $\begin{array}{r}\delta_{F R} \\
\mathrm{~mm}\end{array}$ & $\gamma_{E Q}$ & $\begin{array}{r}\delta_{E Q} \\
\mathrm{~mm}\end{array}$ \\
\hline 1c & 11.93 & 0.00960 & 10.86 & 0.00403 & 4.20 & - & 24.13 \\
2c & 15.10 & 0.00960 & 10.60 & 0.00519 & 4.01 & 0.00121 & 18.92 \\
3c & 18.54 & 0.00960 & 10.22 & 0.00628 & 3.84 & 0.00243 & 16.91 \\
$4 \mathrm{c}$ & 22.25 & 0.00960 & 9.99 & 0.00636 & 3.78 & 0.00396 & 15.84 \\
$5 \mathrm{c}$ & 26.02 & 0.00960 & 9.83 & 0.00603 & 3.74 & 0.00482 & 15.09 \\
$6 \mathrm{c}$ & 29.97 & 0.00960 & 9.79 & 0.00651 & 3.70 & 0.00480 & 15.55 \\
$7 \mathrm{c}$ & 34.09 & 0.00960 & 9.88 & 0.00625 & 3.68 & 0.00546 & 17.96 \\
8c & 38.07 & 0.00960 & 10.22 & 0.00706 & 3.60 & 0.00527 & 20.57 \\
$9 \mathrm{c}$ & 42.91 & 0.00960 & 10.27 & 0.00479 & 3.82 & - & 17.82 \\
\hline \hline
\end{tabular}

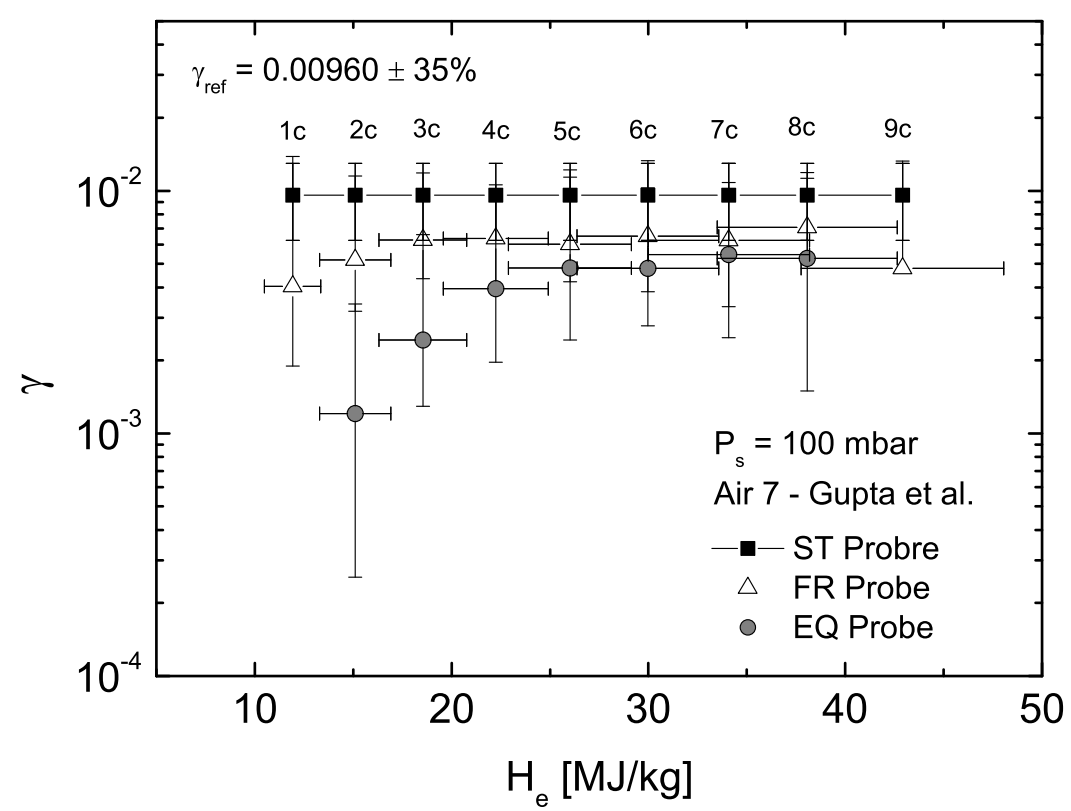

Figure 4.18.: Recombination coefficients on the Damköhler probes at 100 mbar.

Following the same analysis as before, the reduction of the recombination coefficient can be explained with the non-dimensional analysis of different non-equilibrium boundary layers. Again, reducing the size of the probe reduces the computed $D a_{g}$ and, conversely, a larger probe leads to a higher $D a_{g}$, as seen in Fig. 4.19. In addition, comparing with the results obtained at 50 mbar, rising the pressure also increases $D a_{g}$.

Both $D a_{w}$ and $N u$ are shown in Fig. 4.20a and Fig. 4.20b. The same conclusions reached at 50 mbar also apply at 100 mbar. For the Frozen probe, a reduction of both $D a_{g}$ and $N u$ with respect to the Standard device leads to a reduction on $D a_{w}$ and, consequently, to a lower $\gamma$. 


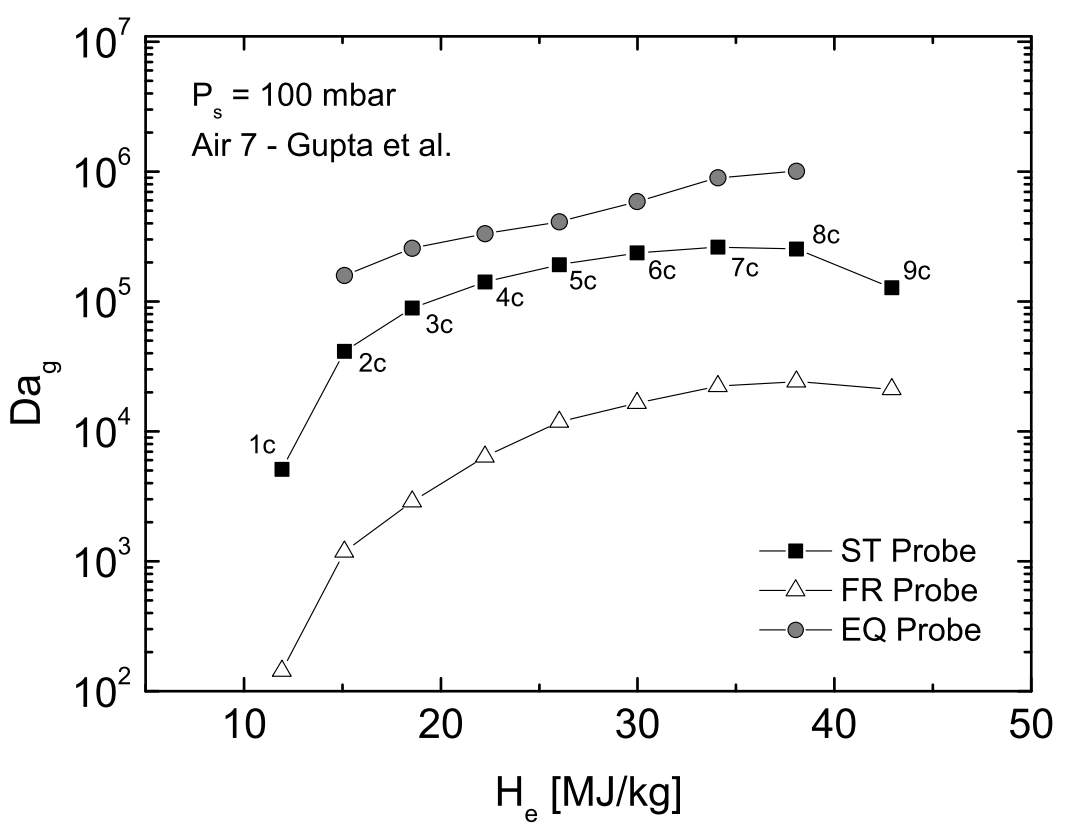

Figure 4.19.: $D a_{g}$ on the Damköhler probes at 100 mbar.

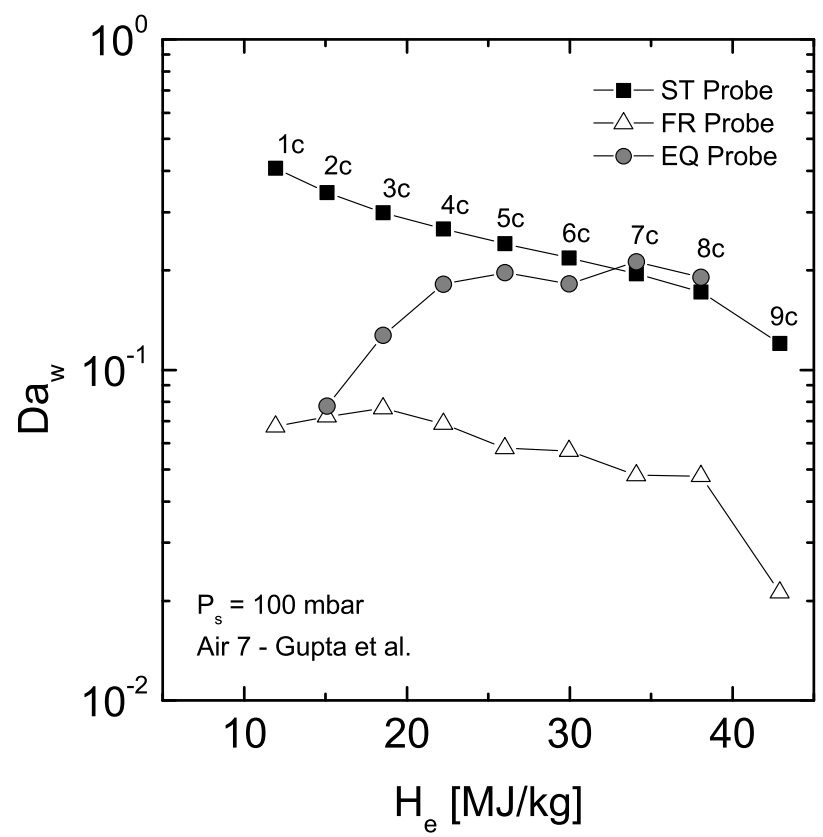

(a) $D a_{w}$ on the Damköhler probes.

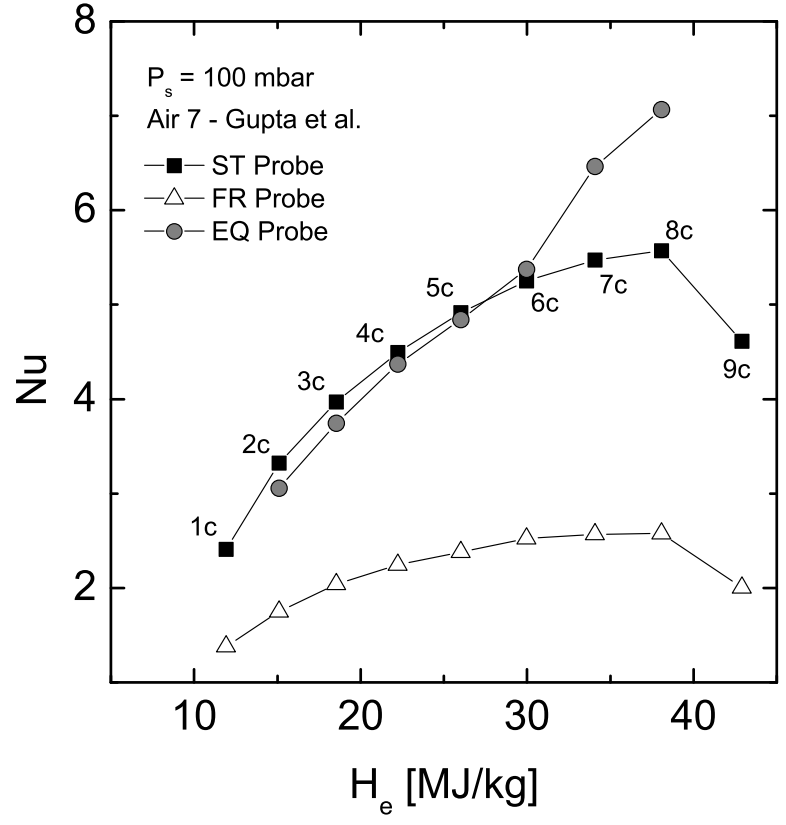

(b) $N u$ on the Damköhler probes.

Figure 4.20.: Non-dimensional numbers obtained at 100 mbar.

For the Equilibrium probe, an increase of $D a_{g}$ while keeping the same $N u$ as in the Standard probe, requires $D a_{w}^{E Q}<D a_{w}^{S T}$ in order to be consistent with Fig. 4.15 , which results into $\gamma_{E Q}<\gamma_{\text {ref }}$ for conditions $2 \mathrm{c}$ to $6 \mathrm{c}$. Therefore, the non-equilibrium influence on the experimentally determined 
recombination coefficient is also present at 100 mbar.

Note that results of the non-dimensional analysis are available in Tables C.1 C.3 for the three pressure conditions under consideration.

\subsection{Catalysis determination at off-stagnation point}

A test campaign is carried out in the Plasmatron to observe the catalytic phenomenon at offstagnation point configuration and to complement the tests on the Damköhler probes. The flat plate described in Sec. 4.2 .2 is used in combination with a non-equilibrium Navier-Stokes solver to study the catalytic behavior at off-stagnation point. The process is similar to the followed for the stagnation point configuration. That is, the comparison of the heat flux measured on the linear calorimeter and the heat flux predicted by the solver are matched to obtain information about the surface catalytic behavior during the experiment.

The test configuration is shown in Fig. 4.21. For each test condition, both the dynamic pressure and the heat flux on the Standard probe are measured to rebuild the boundary layer outer edge properties, so that the reference free-stream conditions in the chamber can be defined. The three probes are installed $445 \mathrm{~mm}$ from the torch exit and properly aligned with the axis of the jet. Both calorimiter holders (Standard and flat plate) are installed on the injection system at the bottom of the chamber, while the pitot probe is located at the top. The pipes from both calorimeters are routed outside the Plasmatron and connected to rotameters that keep the mass flow rate of water constant. The 9 E-thermocouples from the flat plate and the other two from the Standard probe are connected to the data acquisition system and measurements are taken at $1 \mathrm{~Hz}$. Remark that, while the heat flux computation is done in real time for the Standard probe, the measurement on the flat plate requires a more elaborate post-processing (as explained below). The dynamic pressure transducer, which is calibrated with a water manometer, is connected to the static pressure line on one port and to the total pressure signal coming from the pitot probe on the other. The output voltage from the transducer, sensing the pressure difference between the two ports (i.e. $P_{d y n}$ ), is converted into a pressure reading by the data acquisition system containing the calibration curve.

The test envelope for the flat plate is much smaller than for the stagnation point probes. This is due to the larger residence time of the water circulating through both the cooling of the holder and the linear calorimeter. Indeed, the $9^{\text {th }}$ thermocouple of the calorimeter should be observed closely during the probe exposition to the plasma in order to ensure that the circulating water is far enough from its boiling condition. This is the reason why only two test conditions are targeted for this campaign. Since the system temperature rises quite fast in this test configuration, not many measurements can be taken with the flat plate in a single run of the Plasmatron. Conditions $4 \mathrm{~b}$ and 4c from both the Mini-Max and the Damköhler probe testing campaigns are considered the most adequate given the flat plate limitations. Because the purpose of the experiment is focused on the observation of the catalytic behavior rather than an accurate quantification for copper catalysis, the use of only two free-stream conditions is not deemed a major limitation to extract relevant conclusions from the experiments.

To run the experiment, the plasma is switched on and the air mass flow is set to $16 \mathrm{~g} / \mathrm{s}$. Then, the static pressure is set to 50 mbar and the Standard probe is placed into the jet. Electric power $P W$ 


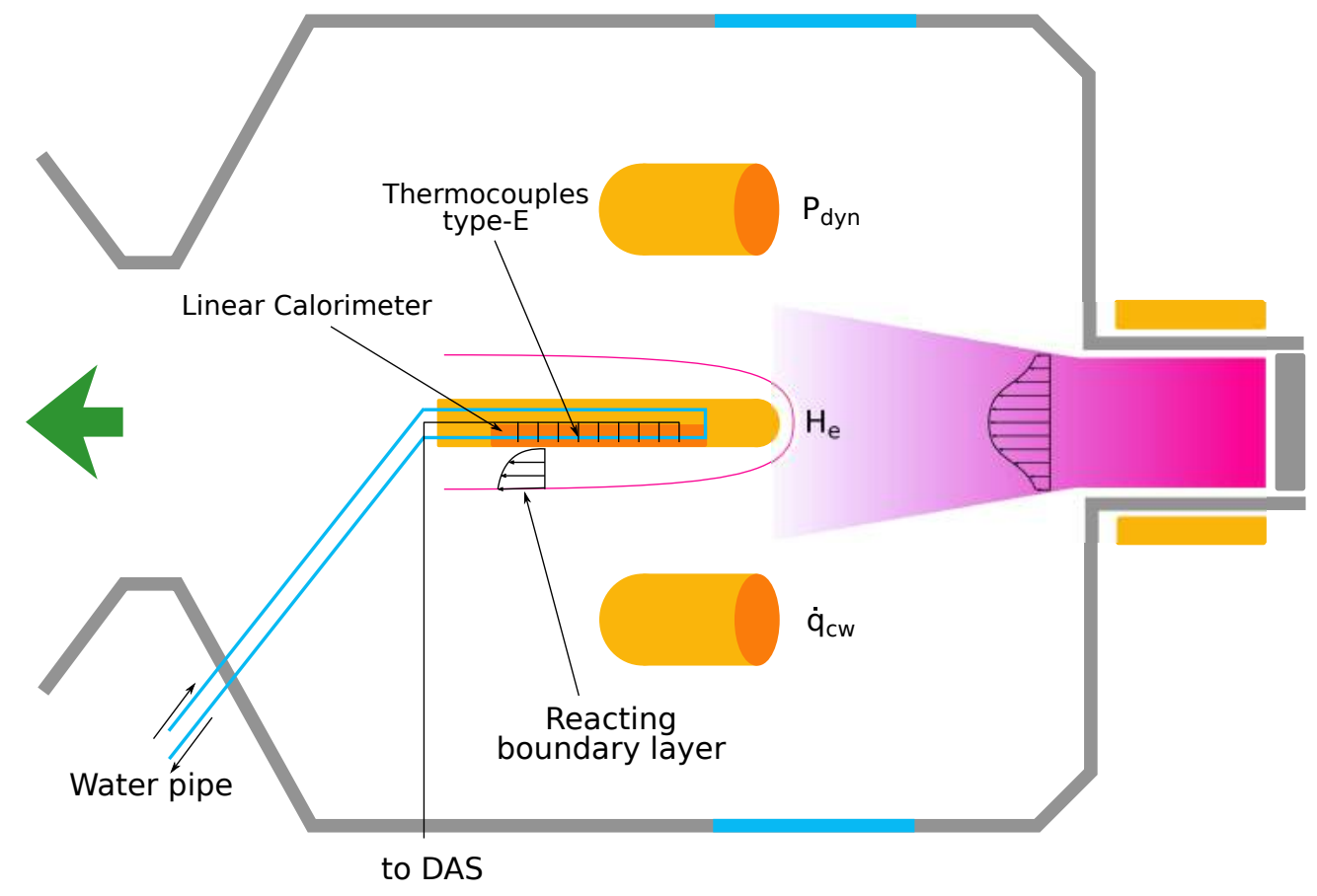

Figure 4.21.: Plasmatron test configuration for catalysis determination on the flat plate.

is increased until the heat flux reaches $900 \mathrm{~kW} / \mathrm{m}^{2}$ in steady state. After the measurement is taken, the dynamic pressure probe is exposed to the jet to measure $P_{d y n}$. These two measurements allow the rebuilding of the free-stream flow. Then, the flat plate is finally introduced into the plasma and the 9 temperature measurements are recorded. Once a steady state condition is reached on all the signals, the flat plate is ejected and $P_{s}$ is increased to 100 mbar. The measurements are repeated for the new test conditions. The $P W$ settings on test $4 \mathrm{~b}$ and $4 \mathrm{c}$ are 221 and $188 \mathrm{~kW}$, respectively. The time-averaged temperatures of the thermocouples on the linear calorimeter, together with their distance from the leading edge, are given in Table 4.7 and plotted in Fig. 4.22 .

Table 4.7.: Thermocouple locations from the leading edge and their time-averaged temperature measurements along the linear calorimeter

\begin{tabular}{rrrrrrrr}
\hline \hline$i$ & $\begin{array}{r}x_{i} \\
{[\mathrm{~mm}]}\end{array}$ & $\begin{array}{r}T_{i}^{(4 b)} \\
{\left[{ }^{\circ} \mathrm{C}\right]}\end{array}$ & $\begin{array}{r}T_{i}^{(4 c)} \\
{\left[{ }^{\circ} \mathrm{C}\right]}\end{array}$ & $i$ & $\begin{array}{r}x_{i} \\
{[\mathrm{~mm}]}\end{array}$ & $\begin{array}{r}T_{i}^{(4 b)} \\
{\left[{ }^{\circ} \mathrm{C}\right]}\end{array}$ & $\begin{array}{r}T_{i}^{(4 c)} \\
{\left[{ }^{\circ} \mathrm{C}\right]}\end{array}$ \\
\hline 1 & 49 & 21.65 & 19.88 & 6 & 165 & 55.21 & 45.02 \\
2 & 62 & 32.70 & 28.84 & 7 & 190 & 59.53 & 47.47 \\
3 & 88 & 43.28 & 38.02 & 8 & 216 & 64.42 & 51.08 \\
4 & 113 & 49.18 & 41.87 & 9 & 229 & 67.65 & 51.40 \\
5 & 139 & 50.99 & 42.25 & & & & \\
\hline \hline
\end{tabular}

The heat flux distribution $\dot{q}_{w}(x)$ along the plate should be derived from the time-averaged temperatures of Fig. 4.22. The fact that experiments provide discrete data of temperature along the $x$-coordinate makes the measured heat flux distribution discontinuous. This issue can be addressed interpolating between the data points, leading to an analytic expression of $T(x)$ from which $\dot{q}_{w}(x)$ 


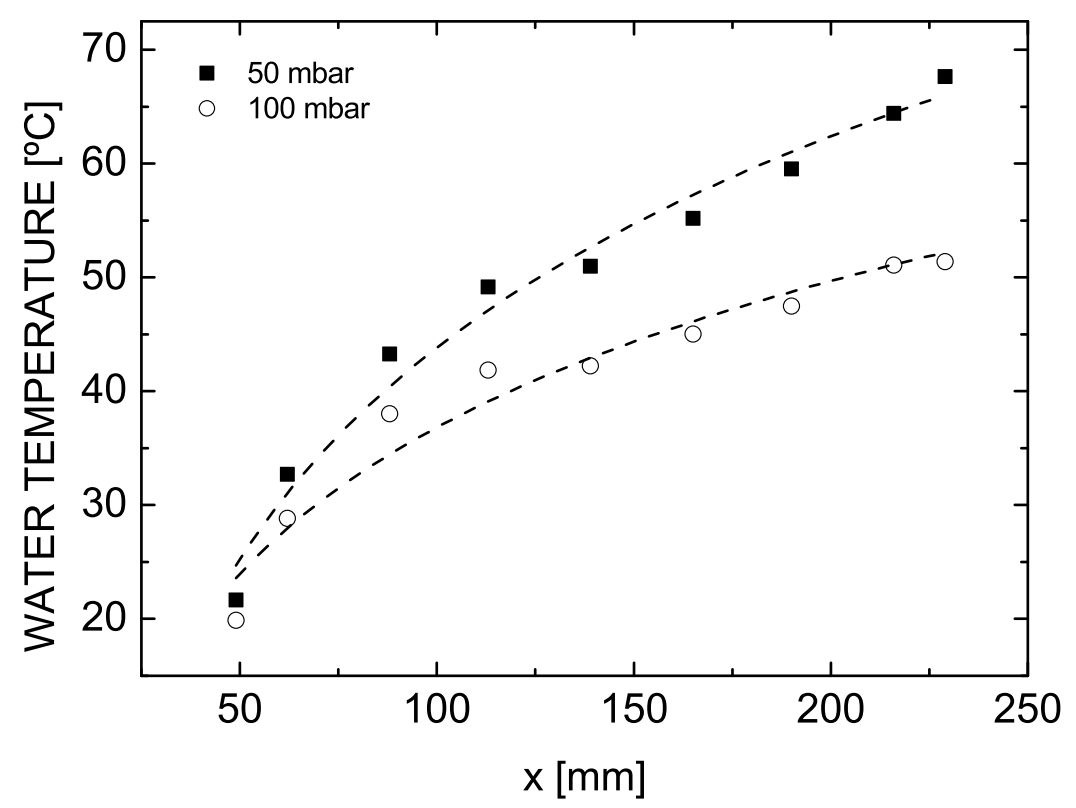

Figure 4.22.: Water temperature distribution along the linear calorimeter.

could be properly derived following:

$$
\dot{q}_{w}(x)=\frac{c_{p} \dot{m}_{H_{2} O}}{d} \frac{\partial T}{\partial x}(x)
$$

where $d=30 \mathrm{~mm}$ is the calorimeter width and $\dot{m}_{\mathrm{H}_{2} \mathrm{O}}=5.093 \mathrm{~g} / \mathrm{s}$ is the water mass flow circulating through the pipes of the calorimeter. Thus, a logarithmic interpolation in the form $T(x)=A \ln (x)+$ $B$ is carried out over the experimental points and constants $A$ and $B$ are provided in Table 4.8 together with the coefficient of determination $\mathrm{R}^{2}$ of the fit.

Table 4.8.: Regression coefficients for $T(x)=A \ln (x)+B$

\begin{tabular}{lrrr}
\hline \hline Test & $A$ & $B$ & $\mathrm{R}^{2}$ \\
\hline $\mathrm{a}$ & 26.801 & -79.601 & 0.9795 \\
$\mathrm{~b}$ & 18.571 & -48.704 & 0.9554 \\
\hline \hline
\end{tabular}

The experimental heat flux measurement should be coupled with CFD in order to extract information about catalysis. The solver used for that purpose is CFD ++ 14.1.1 from Metacomp Technologies. The 2D Navier-Stokes equations under chemical non-equilibrium are solved in a mesh with 14,950 quadrilateral cells around the flat plate geometry. The $2 \mathrm{D}$ solution is computed on the plane defined by the stagnation line. The flow inlet of the mesh is located $287.5 \mathrm{~mm}$ from the leading edge of the flat plate. Velocity, temperature and species profiles are prescribed at the inlet for the simulations. Static pressure is fixed at the upper and rear outlets of the mesh because the flow is subsonic. The wall of the flat plate is assumed isothermal at $350 \mathrm{~K}$. This is reasonable 
given the fact that water temperature increases only $\approx 50 \mathrm{~K}$ along the calorimeter, which is very small compared to the variation of temperature across the boundary layer. The same recombination coefficient $\gamma$ is imposed for both $\mathrm{O}+\mathrm{O} \longrightarrow \mathrm{O}_{2}$ and $\mathrm{N}+\mathrm{N} \longrightarrow \mathrm{N}_{2}$ reactions, with non-catalytic conditions being imposed for the rest of the reactions. The 7 species air model and Gupta et al. [2] chemical model are used to solve the species equations. Note that the same chemical model was used in the previous campaigns of this dissertation. The flow is assumed laminar since the typical Reynolds numbers obtained in the Plasmatron under such configurations are below 100.

It is clear that the inlet conditions of the simulation in $\mathrm{CFD}++$ should match the testing conditions as close as possible. Using the heat flux on the Standard probe and the dynamic pressure measurements taken before introducing the flat plate, one can rebuild with CERBOULA the flow properties at the outer edge of the boundary layer. These values are listed in Table 4.9. However, using this information as inlet conditions for the $\mathrm{CFD}++$ simulations is not correct because the outer edge and the mesh inlet are not located at the same distance from the stagnation point of the probe, which means that a spatial extrapolation is required. Additionally, to properly recreate the same flow environment, it is also important to consider that the inlet is not uniform and that a jet profile develops from the torch exit. Therefore, on one hand an extrapolation along the stagnation line is carried out using the rebuilt data from CERBOULA as reference, and on the other a jet profile is rebuilt on the direction perpendicular to the stagnation line. ICP simulations in CoolFluid are used to assist in such double extrapolation exercise. Again, a 50\% power efficiency is assumed. With the boundary layer outer edge data from CERBOULA as reference point, and the derivatives in the $x$ and $y$ coordinates from the ICP solutions, the temperature and the velocity profiles to prescribe at the inlet of the CFD ++ domain are determained; they are shown in Fig. 4.23 and Fig. 4.24, respectively. The same technique is applied for each of the species.

Table 4.9.: Rebuilt outer edge conditions on the Standard probe

\begin{tabular}{lrrr}
\hline \hline Test & $\begin{array}{r}H_{e} \\
\mathrm{MJ} / \mathrm{kg}\end{array}$ & $\begin{array}{r}T_{e} \\
\mathrm{~K}\end{array}$ & $\begin{array}{r}U_{e} \\
\mathrm{~m} / \mathrm{s}\end{array}$ \\
\hline $4 \mathrm{~b}$ & 19.31 & 5628 & 234 \\
$4 \mathrm{c}$ & 19.91 & 5841 & 103 \\
\hline \hline
\end{tabular}

The heat flux distributions along the flat plate are shown in Fig. 4.25 and Fig. 4.26 for conditions $4 b$ and $4 c$, respectively. An uncertainty of $\pm 10 \%$ is assumed following Eq. 3.26. Measurements are compared to the heat flux distributions predicted by the $\mathrm{CFD}++$ simulations under full-catalytic, non-catalytic and partially catalytic conditions. The experimental values are bracketed between the two numerical results. An evolution from high to low catalytic activity is observed along the linear calorimeter for both test conditions. As it was the case for the stagnation point using the Damköhler probes, the results on the flat plate also show that non-equilibrium characteristics of the boundary layer influence the experimental determination of the recombination coefficient. 


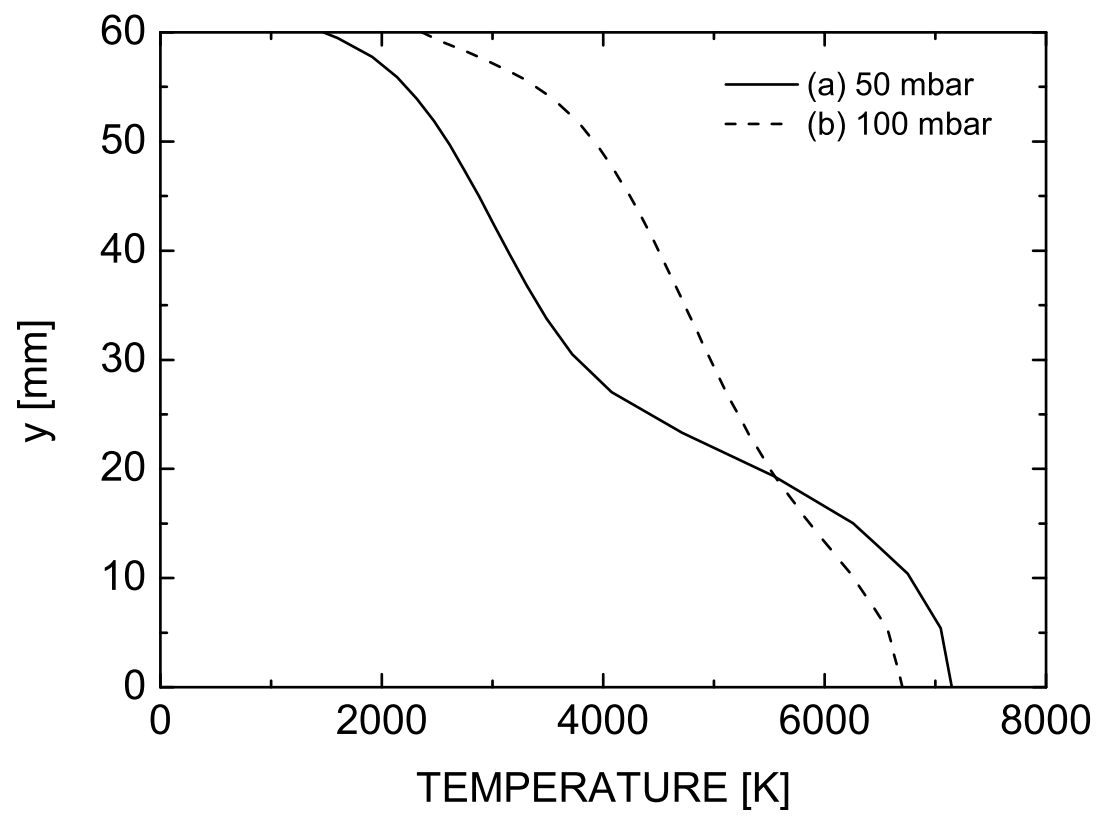

Figure 4.23.: Temperature profiles generated with ICP simulations and imposed as inlet boundary condition in $\mathrm{CFD}++$ computations.

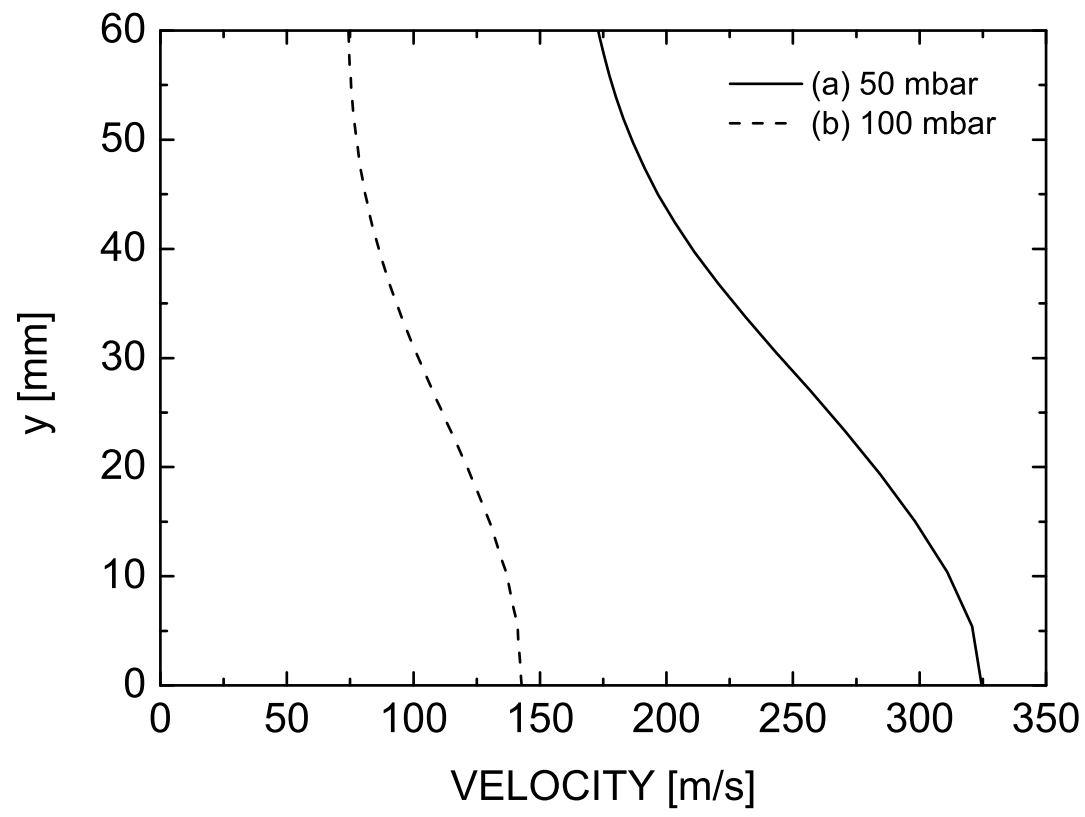

Figure 4.24.: Velocity profiles generated with ICP simulations and imposed as inlet boundary condition in $\mathrm{CFD}++$ computations. 


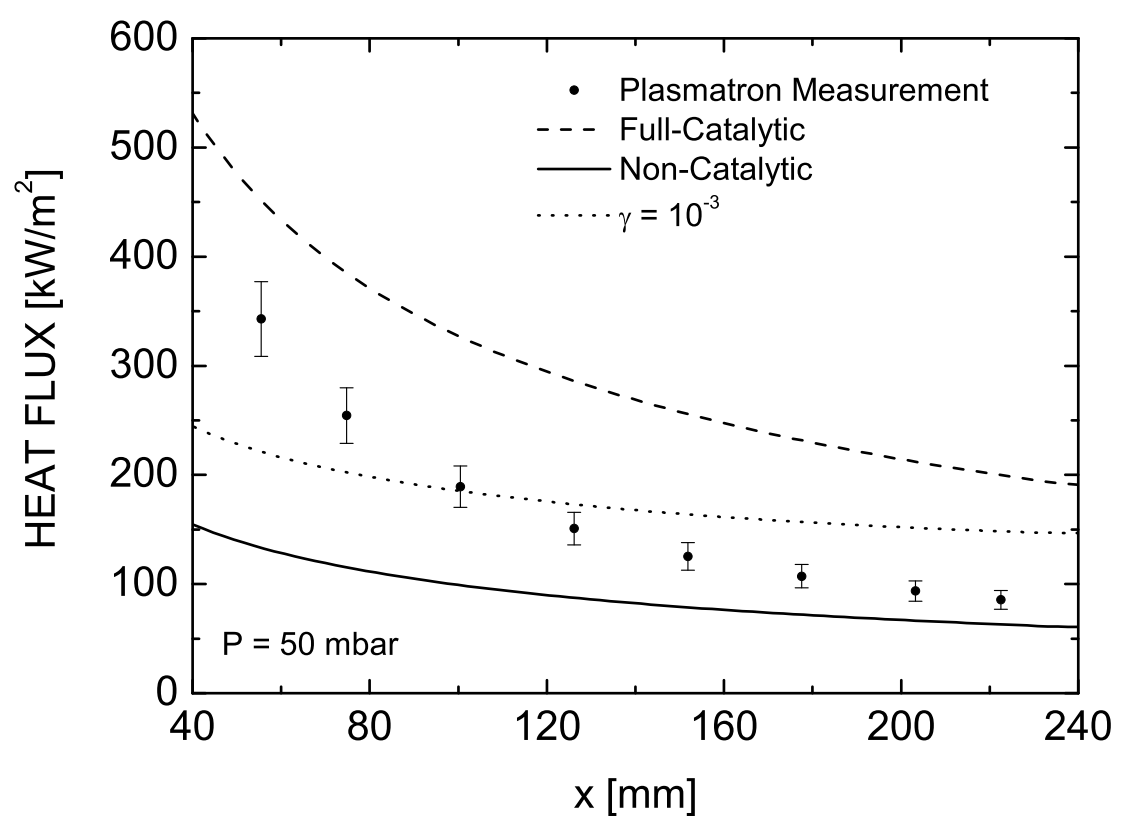

Figure 4.25.: Heat flux distribution along the calorimeter at 50mbar.

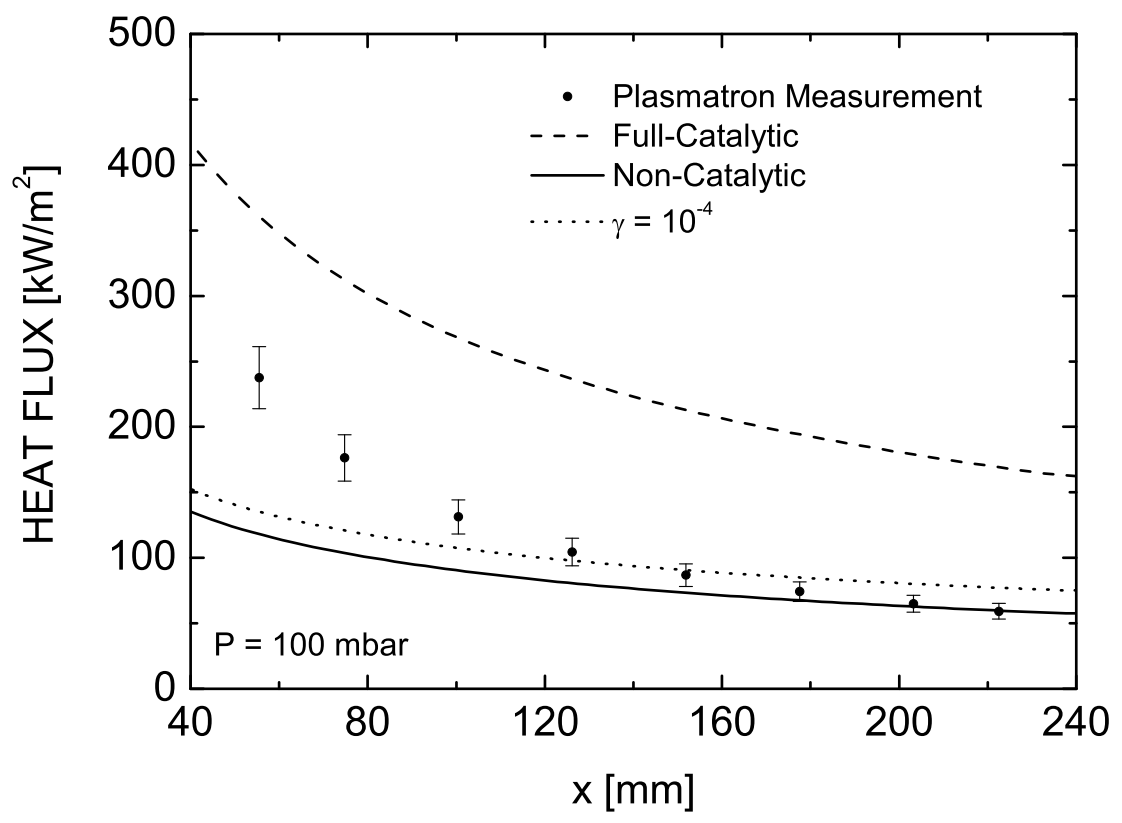

Figure 4.26.: Heat flux distribution along the calorimeter at 100mbar.

Indeed, while the strategy of the Damköhler probes is to modify the non-equilibrium conditions by changing the probe geometry, here the conditions evolve with the boundary layer growth along the flat plate. In fact, it is seen that the increase in $\delta$ could lead to a higher $D a_{g}$ at the downstream end of the plate, and this would cause a recombination coefficient reduction. Note that the dimensional analysis carried out before cannot be directly applied at off-stagnation conditions, mainly because 
the free-steam enthalpy evolves along the flat plate, and this changes the chemical energy exchange parameter $H$ downstream the stagnation point.

The fact that the experimental heat flux transitions from high catalytic to low catalytic along the flat plate makes the accurate quantification of the recombination coefficient very difficult, mainly because the off-stagnation point configuration requires determination of a distribution function $\gamma(x)$ that matches the $\dot{q}_{c w}(x)$ instead of a single value of $\gamma$, and the CFD ++ software does not include this feature yet. Nonetheless, and for the sake of a qualitative assessment of the results, a map of $\dot{q}_{c w}(x)$ is generated using several finite catalytic conditions from non-catalytic to full-catalytic conditions (as in Fig. 4.25 and Fig. 4.26), so that its intersection with the experimental heat flux obtained along the plate leads to the $\gamma(x)$ shown in Fig. 4.27. Note that, although this procedure provides a reasonable estimate of the recombination coefficient distribution, the method is only approximate because it relies on simulations with constant $\gamma$ values along the flat plate, which do not consider the influence that a non-uniform catalytic distribution could have on the flow field.

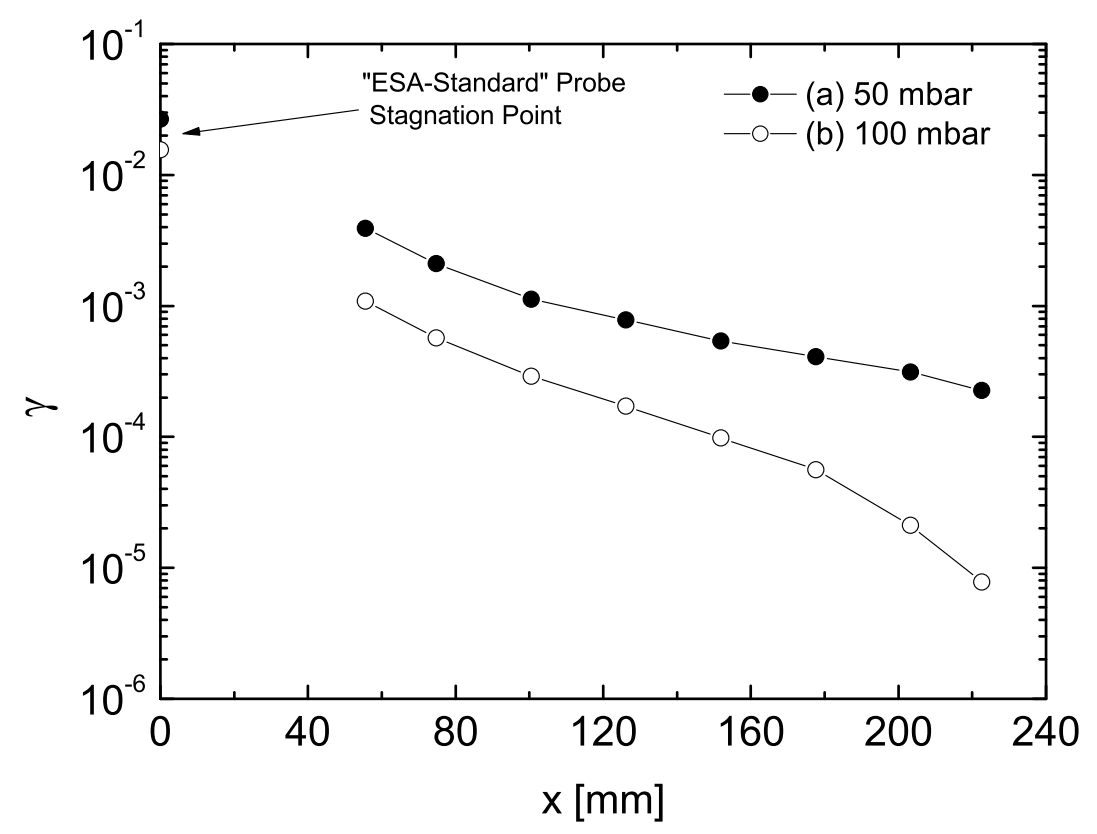

Figure 4.27.: Recombination coefficient distribution along the linear calorimeter.

Remark that dividing the wall boundary in several elements and introducing the discrete values of $\gamma$ reported in Fig. 4.27 leads to a heat flux distribution that, despite showing a similar trend to the measured heat flux, is full of discontinuities and does not represent the physical problem at hand. A better approximation could be obtained by reducing the size of the elements at the wall to introduce $\gamma(x)$ with a higher spatial resolution. However, this is not a practical procedure because it would require a thorough mesh refinement in the direction parallel to the wall for the species gradients caused by catalytic jumps along the plate to be properly reproduced. It is worth noting that previous studies in the VKI have shown that reaching a converged solution in CFD simulations with a catalytic jump is rather difficult. 


\subsection{Concluding remarks}

The results reported in this chapter show that the testing environment has a non-negligible influence on the determination of the wall catalytic activity in the VKI-Plasmatron facility. They are consistent with the analysis proposed by Rosner [99], in which he showed that the Nusselt number of a reacting boundary layer can be expressed as function of the Damköhler numbers evaluated at both the gas and the wall, and of a chemical energy exchange parameter $H$. If the latter remains constant, any non-equilibrium boundary layer configuration should belong to the same surface $N u=f\left(D a_{g}, D a_{w}\right)$. A parametric analysis with the VKI non-equilibrium boundary layer solver (CERBOULA) shows that the same trend of $N u=f\left(D a_{g}, D a_{w}\right)$ is expected among the different testing conditions that can be reached in the Plasmatron chamber.

Two testing campaigns are carried out in the Plasmatron to investigate the influence of the non-equilibrium characteristics of the boundary layer on the experimental quantification of the recombination coefficient. The first one uses the change in size among the Damköhler probes to modify the thickness of the boundary layer in front of a copper calorimeter, the recombination coefficient of which is determined with CERBOULA. The second campaign assesses how the boundary layer development along a copper flat plate can also affect the determination of $\gamma$ when assisted with CFD computations. The recombination coefficient determination on the Damköhler probes not only demonstrates that $\gamma$ is a result of the non-equilibrium configuration of the boundary layer, but also validates the dimensional analysis shown at the beginning of the chapter. Although the recombination coefficient determined on the flat plate is only considered a qualitative result due to the fact that $\gamma$ is reduced downstream of the plate, it shows a behavior similar to the stagnation point measurement. That is, an increase of the boundary layer thickness should bring a reduction of $\gamma$.

The relevance of these results lay on the fact that the recombination coefficient determined in a plasma wind tunnel is influenced not only by surface temperature and static pressure, as reported in the previous chapter and by other authors in literature [107], but also by the whole boundary layer configuration. They also imply that the recombination coefficient being normally used as a boundary condition at the catalytic wall is, in fact, the consequence of a coupling between the surface chemical properties and the flow around the sample. Note that this does not mean that the $\gamma$-model is obsolete, but that care should be taken when changing configurations. As a final remark, this issue might not arise with FRC models because the recombination coefficient is determined by the different processes taking place at the surface of the catalytic sample. 



\section{Chapter 5.}

\section{Flight extrapolation at off-stagnation point}

In the framework of surface kinetics characterization, and more particularly, the quantification of the catalytic recombination coefficient of atomic species at the wall of a re-usable TPS, the proper non-equilibrium boundary layer reproduction is not systematically taken into account. In fact, consistently with the first order reaction hypothesis, only surface temperature is normally considered to define the catalytic reaction rates. However, results previously obtained in this thesis, and in Ref. [41, 42, 107, 109] show that the recombination coefficient determination in plasma wind tunnels is influenced by both pressure and boundary layer chemical non-equilibrium conditions around a sample. This is the reason why flight to ground extrapolation methodologies are required, not only for spacecraft design, but also for validation of gas-surface interaction models when specific flight data is available.

The stagnation point is normally taken into account as a TPS design reference because it is considered as the most critical location in terms of heat flux. Indeed, this explains why most literature is dedicated to measurements under stagnant flow configurations [11, 67, 110]. The flightto-ground extrapolation methodology proposed by Kolesnikov [7, 8] under the LHTS concept, as explained in Sec. 2.3, completely duplicates the physics within the stagnation point boundary layer developed in front of a cylindrical body flying in hypersonic regime, leading to a valid strategy to relate the measured $\gamma$ on ground with the operating situation in flight.

However, transition to turbulence, catalytic jumps caused by adjacent patches of different materials or body flap deflection in lifting body concepts, are all examples of off-stagnation point phenomena increasing the heat transferred to the vehicle. Therefore, a proper flow analysis at off-stagnation conditions is critical to improve the TPS design throughout the vehicle.

A flight-to-ground extrapolation at off-stagnation point is introduced by Barbante [111] using simple geometries, for instance between two spheres or between two flat plates. This chapter provides a flight-to-ground extrapolation methodology that locally duplicates on a flat plate the offstagnation point boundary layer found over a flying body with a generic geometry. It is important to remark that, in this context, local duplication means that the extrapolation is only applied to a certain point of the flying body rather than to the whole boundary layer growth and evolution around the vehicle.

\subsection{The off-stagnation point extrapolation methodology}

Barbante [111 developed a methodology for off-stagnation point based on the boundary layer equations for generic 2-dimensional and for axisymmetric body configurations using the Lees- 
Dorodnitsyn coordinate transformation:

$$
\begin{gathered}
\xi=\int_{0}^{s} \rho_{e} \mu_{e} u_{e} r^{2 \epsilon} d s \\
\eta=\frac{u_{e} r^{\epsilon}}{\sqrt{2 \xi}} \int_{0}^{y} \rho d y
\end{gathered}
$$

Assuming local self-similarity, that is neglecting the derivatives with respect to $\xi$ in front of the derivatives with respect to $\eta$ (except for pressure), the following heat flux equation is obtained:

$$
\begin{aligned}
\dot{q}_{w}=0.332 & \frac{\rho_{e} \mu_{e}}{\operatorname{Pr}^{2 / 3}} f_{c}\left(h_{0, e}-h_{0, w}\right)\left\{1+\frac{u_{e}^{2}(P r-1)}{4\left(h_{0, e}-h_{0, w}\right) P r^{1 / 4}}\right. \\
& \left.+\frac{L e-1}{L e^{2 / 3}}\left[1+0.47\left(\frac{P r}{L e}\right)^{1 / 3} \frac{1}{D a_{w}}\right] \frac{\left[c_{A e}+D a_{g} G_{e}\right] \Delta h_{A}}{h_{0, e}-h_{0, w}}\right\}
\end{aligned}
$$

Note that $\rho, \mu, h_{0}$ and $u$ are the mixture density, mixture viscosity, total enthalpy $\left(h+u^{2} / 2\right)$ and velocity, respectively; with the subscripts $e$ and $w$ referring to the property evaluated at the boundary layer edge and at the body wall. $P r$ and $L e$ are both the Prandtl and Lewis numbers, while $D a_{g}$ and $D a_{w}$ are the gas-phase and the heterogeneous Damköhler numbers, respectively. The term $G_{e}$ is an integral effect of the gas-phase chemical reactions in the boundary layer, $c_{A}$ is the atomic mass fraction, and $\Delta h_{A}$ is the formation enthalpy of atomic species. $f_{c}$ is the flight condition factor, defined as:

$$
f_{c}=\frac{u_{e} r^{\epsilon}}{\sqrt{\xi}}
$$

It is an integral parameter that collects the physics of the boundary layer from the stagnation point $(s=0)$ to the wall coordinate $s$ at off-stagnation point through its dependence with $\xi$.

Similarly to the LHTS concept, if all the parameters present in Eq. 5.3 are equal in flight and ground, the same heat flux should be obtained in the two configurations. However, the measurement of some of these variables in a plasma wind tunnel could be impractical. This is the reason why other quantities, albeit equivalent, should be considered for the application of the methodology. Note first that the 13 parameters proposed in the heat flux equation could be classified as in Table 5.1 .

Table 5.1.: List of variables appearing in Eq. 5.3

\begin{tabular}{lc}
\hline \hline Flow variables: & $\rho_{e}, \mu_{e}, c_{A e}, u_{e}, h_{0, e}, h_{0, w}$ \\
Chemical model variables: & $G_{e}, \Delta h_{A}$ \\
Non-dimensional numbers: & $P r, L e, D a_{g}, D a_{w}$ \\
Flow topology variables: & $f_{c}$ \\
\hline \hline
\end{tabular}

Chemical model variables $G_{e}$ and $\Delta h_{A}$ are automatically reproduced on ground because the gas is the same. Furthermore, Prandtl and the Lewis numbers are function of thermodynamic parameters, therefore their duplication is guaranteed as long as temperature, pressure and gas composition are 
matched; the same applies to viscosity. This reduces the number of independent variables to be extrapolated at off-stagnation point to 8 . Then, regarding the two Damköhler numbers that can be written as $D a_{g}=k_{g} \delta^{2} / D$ and $D a_{w}=k_{w} \delta / D$, one notices that they can be replaced by the boundary layer thickness $\delta$ and the wall kinetics $k_{w}$, with the latter being directly proportional to the recombination coefficient $\gamma$ (see Eq. 2.3). Finally, thermodynamic parameters such as enthalpy and density could be reproduced through temperature and pressure if the species composition remains the same. Thus, the parameters to be considered for the extrapolation could be simplified and re-arranged as in Table 5.2 .

Table 5.2.: List of independent variables for extrapolation

\begin{tabular}{lc}
\hline \hline Outer edge variables & $T_{e}, P_{e}, c_{e}, u_{e}, \delta$ \\
Wall variables & $\gamma, T_{w}$ \\
Flow topology variables & $f_{c}$ \\
\hline \hline
\end{tabular}

Interestingly, considering LTE at the boundary layer edge, which implies that $c_{e}=f\left(T_{e}, P_{e}\right)$, the LHTS comes out as a particular case of the more general off-stagnation point problem. In fact, $f_{c}$ and $u_{e}$ reduce to the velocity gradient $\beta=\partial u_{e} / \partial x$ and to 0 at stagnation point, respectively.

The off-stagnation point extrapolation applied in Ref. [111] is carried out using simple geometries in flight and ground configurations, such as spheres or flat plates, from which analytic expressions of $f_{c}$ can be derived. The purpose of this chapter is to investigate how a methodology can be applied to extrapolate from a complex lifting body geometry, to a simplified and more adequate flow configuration for testing in ground facilities. In the following lines, a flat plate is used as test model configuration because of the simplicity to derive analytic expressions for $f_{c}$. There are other advantages that justify such choice. The first one is the easy manufacture and instrumentation of the model. The second is the simplicity of the meshing for CFD calculations and further analysis of the flow. The third is the fact that the outer edge values remain constant along the plate. And, last but not least, the flexibility it provides to find the correct location on the test model where the boundary layer profile is locally reproduced.

The extrapolation methodology at off-stagnation point takes the local values of $T_{e}, P_{e}, c_{e}$ and $u_{e}$ at certain location $s$ of the flying body as inlet conditions for the flat plate. Then, there are two alternatives to define the length of the flat plate $L_{F P}$ at the end of which the boundary layer is locally reproduced. One of them is imposing the condition $f_{c}^{(f l i g h t)}=f_{c}^{(F P)}$, leading to discrepancies in heat fluxes, and the other is imposing $\dot{q}_{w}^{(f l i g h t)}=\dot{q}_{w}^{(F P)}$, bringing differences between flight condition factors. The former is more interesting academically, given the strict reproduction of all the terms on the right hand side of Eq. 5.3 followed by the heat flux evaluation, while the latter is more practical given that the final goal of the extrapolation is the heat flux reproduction on ground for TPS material testing. In any case, both options are reasonable for the local duplication of the boundary layer. Furthermore, if the same conditions at the wall of the flying body are imposed also on the flat plate simulations, that is same catalysis and same emissivity $(\varepsilon)$ for a wall in radiative equilibrium, the resulting boundary layer profiles should be the same in flight and ground configurations. Note that $\varepsilon$ replaces $T_{w}$ in Table 5.2 for a wall in radiative equilibrium because, under these conditions, 
heat flux and wall temperature are related through the right hand side of Eq. 1.2, An illustration of the methodology is provided in Fig. 5.1.

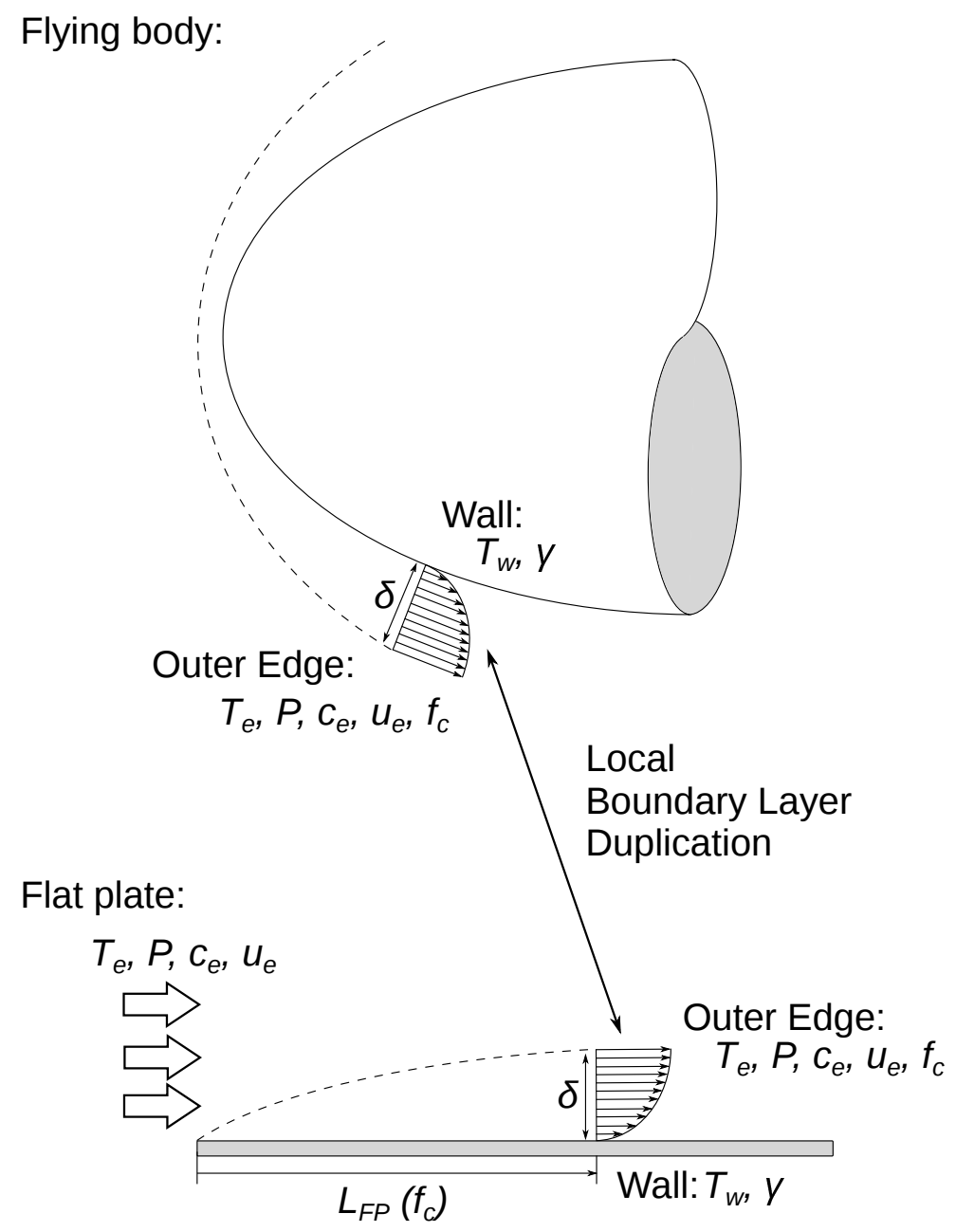

Figure 5.1.: Local off-stagnation point boundary layer duplication concept from a flying body to a flat plate.

Regarding the numerics, it is worth noting that 6 of the independent parameters are already imposed by the boundary conditions, while the other two $\left(T_{w}, \delta\right)$ are the output variables from the solution of the flow equations, and hence, determine the flow topology that is finally obtained on the flat plate.

It seems obvious that the application of the extrapolation as explained above can lead to an $L_{F P}$ that is in the same order of magnitude as the wall coordinate $s$ on the flying body. This means that the methodology, as it is, becomes impractical on ground for points in the flying body that are too far from the stagnation point. This is due to two main reasons related to typical plasma wind tunnel operating conditions. On one side, there is a limitation on the size of the model that can be installed in a test chamber and, on the other, the $u_{e}$ of the vehicle might be too large to match the test conditions of the facility. Therefore, a second extrapolation step must be included so that the boundary layer is scaled to match the restrictions imposed by the plasma wind tunnel.

The scaling procedure is proposed by Barbante [111. It consists on duplicating the boundary layer between two flat plates of different scales. The main difference with respect to the first step 
of the methodology is that, between two plates, the boundary layer can be reproduced not only locally but also globally. That is, the boundary layer development is the same from the stagnation point to the rear end of each model. The logic behind the scaling procedure lies on the fact that the flight condition factor reduces to $\sqrt{u_{e} / s}$ when extrapolating between two plates. Remark that the inverse of the parameter inside the squared root can be understood as a characteristic convective time of the flow in this configuration. However, one can note that respecting both $u_{e} / s$ and $u_{e}$, as required by the first step of the extrapolation, means that the scaling procedure can not be directly applied. In order to overcome this issue, the application of such scaling method could be limited to low Eckert numbers $\left(u_{e}^{2} / h_{e}<<1\right)$, which assumes that the kinetic energy of the flow is negligible compared to the thermal energy. Note that typical values of the Eckert number can range between 0 at the stagnation point and 0.5 at the rear part of a flying body, and is expected to be low in critical zones considered for TPS design. After this assumption, the duplication requirement of $u_{e}$ can be relaxed so just the static enthalpy should be the preserved parameter instead of the total enthalpy appearing in Eq. 5.3. Then, the inlet velocity to be applied in the reduced model $U_{\text {test }}$ can be defined as:

$$
U_{\text {test }}=\frac{L_{\text {model }}}{L_{F P}} u_{e}
$$

with $L_{\text {model }}$ being the size of the small flat plate to be used in ground.

In summary, the extrapolation from flight to ground of an off-stagnation point boundary layer developed around a flying body can be achieved in two steps, each one addressing certain aspects of the flow configuration. The first one reproduces the local boundary layer at the end of a flat plate, whose size $L_{F P}$ and free stream velocity are of the same order as the wall coordinate $s$ and $u_{e}$ obtained on the flying body. Indeed, with the main difference between the two configuration being the geometry, this step addresses the curvature effects on the flow around the space vehicle. The second step adapts both the size $L_{\text {model }}$ and velocity $U_{\text {test }}$ on the flat plate to the particular plasma wind tunnel requirements. Therefore, it addresses the scaling of the flow for the practical application of the method.

One additional and important remark is that an approximation can be done to simplify the method down to a single extrapolation step. This approximation benefits from the the fact that fluid properties remain constant along the boundary layer edge of the flat plate, so they can be taken out of the integral in the definition of $\xi^{(F P)}$, yielding the flat plate length:

$$
L_{F P} \approx \frac{\xi^{(f l i g h t)}}{\rho_{e} \mu_{e} u_{e}}
$$

Indeed, with this estimation of $L_{F P}$ one could define $U_{\text {test }}$ without the first step of the extrapolation.

This approach is assessed in Sec. 5.2.1, where the methodology is applied for $f_{c}^{(f l i g h t)}=f_{c}^{(F P)}$ and $\dot{q}_{w}^{(f l i g h t)}=\dot{q}_{w}^{(F P)}$ options to define the length of the flat plate. 


\subsection{Application of the methodology}

The extrapolation is applied to the IXV (introduced in Sec. 1.4 with computations carried out using CFD ++ software (version 14.1) from Metacomp Technologies. In order to respect as much as possible the theory explained in Sec. 5.1, its application is restricted to solve the equations as a two-dimensional problem. Although this is not consistent with current numerical procedures, which are mostly based in 3D models, the 2D exercise should provide a better understanding of the possible discrepancies in the physics between flight and ground test cases. The fluid domain is defined by four boundaries and divided in 33,072 quadrilateral cells with the vehicle at $45^{\circ}$ of angle of attack. The free-stream conditions specified in Table 5.3 with subscript $\infty$ are set at the inlet. They correspond to an altitude slightly above $60 \mathrm{~km}$. The wall of the IXV is assumed in radiative equilibrium, with the emissivity $\varepsilon$ and the catalytic recombination coefficient $\gamma$ for both atomic nitrogen and oxygen on $\mathrm{C} / \mathrm{SiC}$ [1] being also provided in Table 5.3. Remark that $\gamma$ is assumed zero for the rest of the species. A zero-gradient outflow condition is prescribed at the outlet and mirror symmetry is used for the stagnation line. The gas mixture under consideration is a 5-species air model and Park's chemical model [3] is used to compute the reaction rates.

Table 5.3.: Boundary conditions of the IXV simulation

\begin{tabular}{lccccc}
\hline \hline$P_{\infty}$ & $T_{\infty}$ & $\begin{array}{c}U_{\infty} \\
\mathrm{ms}^{-1}\end{array}$ & $\begin{array}{c}M_{\infty} \\
\mathrm{Ya}\end{array}$ & $\begin{array}{c}\varepsilon \\
\mathrm{K}\end{array}$ & $\begin{array}{c}\gamma_{N}=\gamma_{O} \\
-\end{array}$ \\
\hline 6.13 & 222.44 & 5980.1 & 20 & 0.8 & 0.00694 \\
\hline \hline
\end{tabular}

The contour line corresponding to $99 \%$ of the specific total enthalpy $(17.7 \mathrm{MJ} / \mathrm{kg})$ is used to define the boundary layer edge. This is a rather convenient procedure because velocity, temperature and species gradients are present throughout the solution domain. Furthermore, the definition of the boundary layer outer edge is not strictly specified by Barbante [111], so there is no restriction in this regard. The boundary layer is analyzed at 5 locations along the wall-coordinate of IXV: (a) $s=40$, (b) $s=60$, (c) $s=80$, (d) $s=100$ and (e) $s=175 \mathrm{~cm}$ from the stagnation point. At each of these points, properties of interest are obtained at both the boundary layer edge and the wall, and they are listed in Table 5.4 . These values are used to set the boundary conditions of the flat plate simulations.

\subsubsection{Curvature effect}

Since the location on the flat plate where the boundary layer shall be locally reproduced is not known a priori, a domain surrounding a flat plate with $2 \mathrm{~m}$ in length is meshed. A thickness of 1 $\mathrm{mm}$ is set with a rounded leading edge. This creates a stagnation point serving as reference to define the location on the flat plate where the IXV boundary layer is duplicated. The inlet conditions are set $1 \mathrm{~m}$ ahead of the stagnation point and a height of $1.5 \mathrm{~m}$ is provided to avoid any kind of flow blockage due to the development of the boundary layer. The flat plate domain is composed of 97,500 quadrilateral cells enclosed by 5 boundaries: inlet, outlet, the wall of the flat plate and two symmetry planes, one at the stagnation line and the other at the top boundary. This flat plate 
Table 5.4.: IXV simulation results at positions (a) through (e)

\begin{tabular}{lccccc}
\hline \hline & $(\mathrm{a})$ & $(\mathrm{b})$ & $(\mathrm{c})$ & $(\mathrm{d})$ & $(\mathrm{e})$ \\
\hline$T_{e}, \mathrm{~K}$ & 5364.8 & 5271.0 & 5340.2 & 5365.7 & 5390.9 \\
$P_{e}, \mathrm{~Pa}$ & 2445.5 & 2219.2 & 2263.9 & 2272.8 & 2265.3 \\
$u_{e}, \mathrm{~m} / \mathrm{s}$ & 1180.8 & 1367.6 & 1360.1 & 1372.4 & 1452.6 \\
$c_{N, e},-$ & 0.1959 & 0.1929 & 0.1904 & 0.1889 & 0.1846 \\
$c_{O, e},-$ & 0.2315 & 0.2317 & 0.2316 & 0.2315 & 0.2314 \\
$c_{O_{2}, e},-$ & $3.57 \times 10^{-5}$ & $2.91 \times 10^{-5}$ & $3.31 \times 10^{-5}$ & $3.55 \times 10^{-5}$ & $3.91 \times 10^{-5}$ \\
$c_{N O, e},-$ & 0.0027 & 0.0023 & 0.0025 & 0.0027 & 0.0028 \\
$c_{N_{2}, e},-$ & 0.5699 & 0.5731 & 0.5754 & 0.5769 & 0.5810 \\
$f_{c}, \mathrm{~m}^{2} / \mathrm{kg}$ & 203193.5 & 172776.6 & 139980.7 & 124401.2 & 94547.8 \\
$T_{w}, \mathrm{~K}$ & 1359.2 & 1275.2 & 1226.0 & 1200.0 & 1127.3 \\
$\delta, \mathrm{mm}$ & 18.4 & 23.9 & 31.0 & 35.3 & 48.9 \\
$\dot{q}_{w}, \mathrm{~kW} / \mathrm{m}^{2}$ & 154.8 & 119.0 & 102.3 & 93.9 & 73.1 \\
\hline \hline
\end{tabular}

domain is intentionally over-sized for the case at hand to accommodate several flight conditions using the same discretization.

The outer edge values $T_{e}, P_{e}, u_{e}$ and $c_{i, e}$ obtained in the IXV simulations are prescribed as input conditions for the flat plate. For subsonic flows, pressure is set at the outlet and the rest of the variables at the inlet. The wall emissivity and catalytic activity are those given in Table 5.3. One flat plate simulation is run for every IXV boundary layer station ((a) through (e)). The boundary layer outer edge is defined as the $99 \%$ of the total enthalpy evaluated at the inlet of each simulation. Properties along the edge are used to compute the flight condition factor distribution throughout the wall coordinate of the plate $\left(f_{c}(s)\right)$. The requirement $f_{c}^{(I X V)}=f_{c}^{(F P)}$ is used to define the location on the flat plate at which the same boundary layer is obtained or, equivalently, the length of the flat plate $L_{F P}$ at the end of which the boundary layer is duplicated. The reproduction of a generic variable $\phi$ at a given condition is tested against the computed value on the IXV simulation. Then, a relative error $\zeta$ is estimated as:

$$
\zeta=\frac{\phi^{(F P)}-\phi^{(I X V)}}{\phi^{(I X V)}}
$$

Figure 5.2 reports $\zeta$ values for the input variables of the methodology, showing that the differences in boundary layer outer edge properties between IXV and flat plate do not exceed $\pm 2.5 \%$.

The relative differences of the output variables $\left(T_{w}, \delta\right)$ with respect the IXV values are reported in Table 5.5, together with the relative differences in heat flux. The largest discrepancies are obtained at location (e) and they do not exceed $4 \%, 16 \%$ and $17 \%$ for $T_{w}, \delta$ and $\dot{q}_{w}$, respectively. Note that only $\delta$ and $T_{w}$ should be considered as output variables of the methodology for the radiative equilibrium conditions at the wall. Instead, the heat flux is regarded as a consequence of the resulting $T_{w}$ obtained from the simulations due to the definition $\dot{q}_{w}=\sigma \varepsilon T_{w}^{4}$. In fact, one could derive from this expression that $\zeta_{\dot{q}_{w}} \approx 4 \zeta_{T_{w}}$, which would explain the significant differences in heat flux reported in Table 5.5, despite the low discrepancies in wall temperatures.

It is worth mentioning also the relation between $\zeta_{\dot{q}_{w}}$ and $\zeta_{\delta}$ being shown in Fig. 5.3 . It is observed 


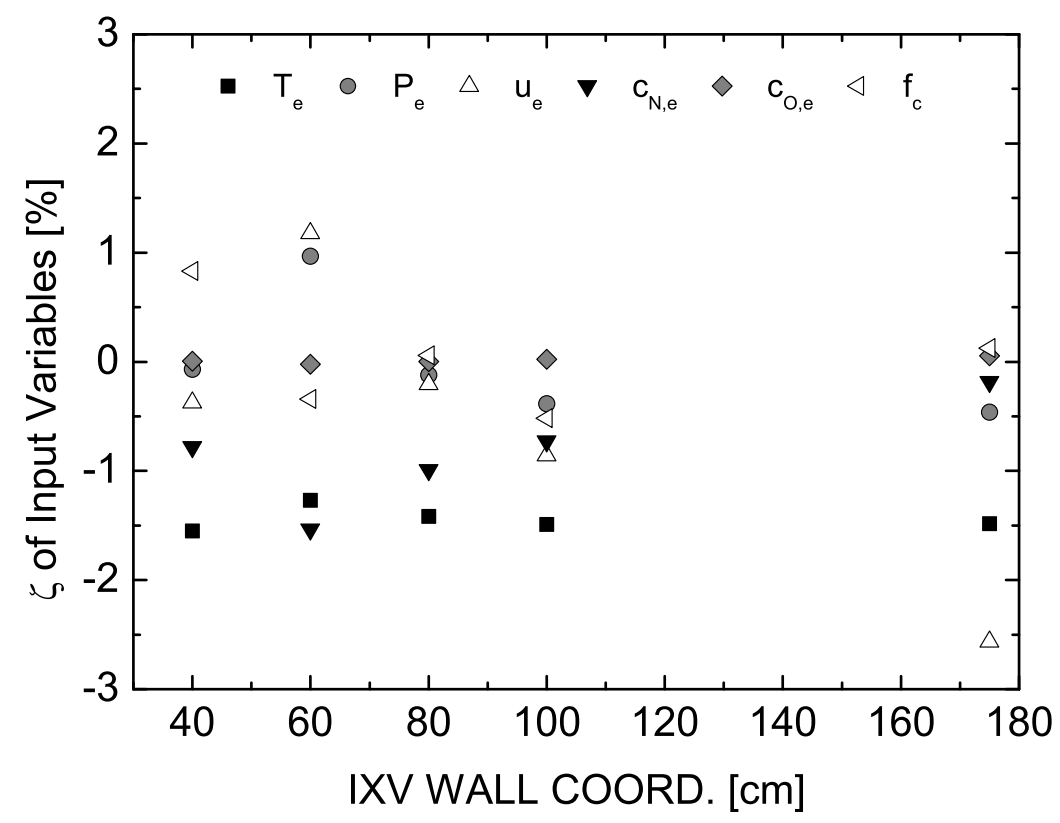

Figure 5.2.: Relative differences of the boundary layer outer edge parameters on the flat plate with respect to IXV.

Table 5.5.: Relative difference of the output variables with respect the IXV, if $f_{c}^{(I X V)}=f_{c}^{(F P)}$

\begin{tabular}{lccccc}
\hline \hline & $(\mathrm{a})$ & $(\mathrm{b})$ & $(\mathrm{c})$ & $(\mathrm{d})$ & $(\mathrm{e})$ \\
\hline$\zeta_{T_{w}}, \%$ & 1.67 & 3.34 & 3.90 & 3.65 & 3.96 \\
$\zeta_{\dot{q}_{w}}, \%$ & 6.71 & 14.78 & 16.55 & 15.47 & 16.85 \\
$\zeta_{\delta}, \%$ & -7.76 & -9.21 & -13.25 & -12.96 & -15.33 \\
\hline \hline
\end{tabular}

that $\zeta_{\delta}$ is always negative, meaning that the boundary layer developed over the flat plate is always thinner than the one developed on IXV, which leads to a higher heat flux on the flat plate. This is reasonable given the fact that the heat flux transferred to the wall depends on the chemical nonequilibrium conditions of the boundary layer through $D a_{g}$ and $D a_{w}$, both depending strongly on $\delta$. A correlation for the heat flux transferred to a catalytic wall through a boundary layer in chemical non-equilibrium was proposed by Rosner [99], who stated $\dot{q}_{w} \propto \delta^{m}$, where $m$ is negative. The same relation is observed in the off-stagnation point extrapolation methodology, so the higher $T_{w}$ on the flat plate model could be seen as consequence of the thinner boundary layer.

The results of the methodology for the $L_{F P}$ approximation proposed in Eq. 5.6 present a similar behavior, as reported in Table 5.6. Moreover, comparing them with the results from $f_{c}^{(I X V)}=f_{c}^{(F P)}$ shows that discrepancies obtained in $T_{w}, \dot{q}_{w}$ and $\delta$ are lower.

An alternate procedure to apply the methodology consists on finding the location on the flat plate at which the heat flux equals that on the IXV. This happens, of course, downstream the point where the $f_{c}$ values are equal. The output variables of this procedure are the flight condition factor and the boundary layer thickness, their relative errors are reported in Table 5.7. Note that, while 


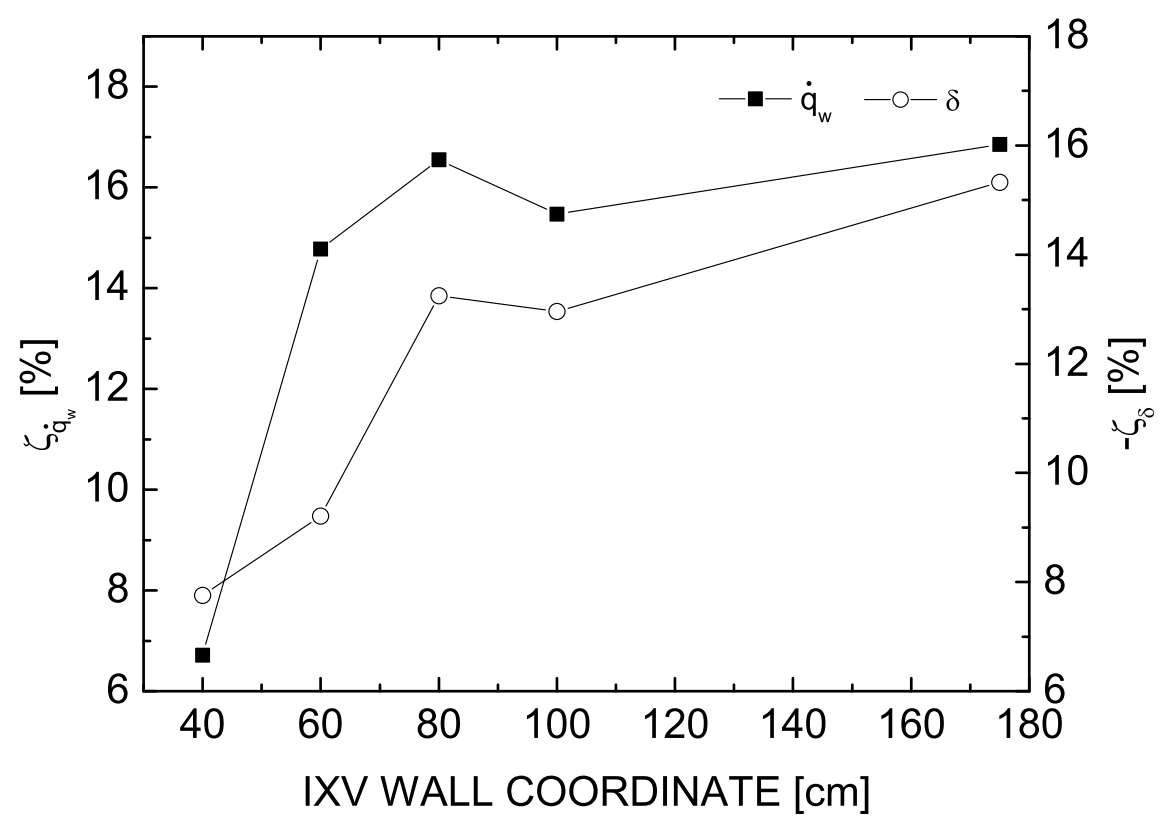

Figure 5.3.: Relative differences of the heat flux and thickness with respect to the IXV, under the same $f_{c}$ at different extrapolating locations.

Table 5.6.: Relative difference of the output variables with respect the IXV, if $L_{F P} \approx \xi^{(I X V)} / \rho_{e} \mu_{e} u_{e}$

\begin{tabular}{lccccc}
\hline \hline & $(\mathrm{a})$ & $(\mathrm{b})$ & $(\mathrm{c})$ & $(\mathrm{d})$ & $(\mathrm{e})$ \\
\hline$\zeta_{T_{w}}, \%$ & 0.60 & 2.41 & 2.67 & 2.38 & 2.34 \\
$\zeta_{\dot{q}_{w}}, \%$ & 2.29 & 10.68 & 11.16 & 9.91 & 9.72 \\
$\zeta_{\delta}, \%$ & -1.08 & -4.11 & -7.35 & -7.09 & -8.63 \\
\hline \hline
\end{tabular}

the boundary layer is always thinner when enforcing the same $f_{c}$, the thickness obtained on the flat plate simulations when prescribing the same $\dot{q}_{w}$ is larger than on the IXV $\left(\zeta_{\delta}\right)$. This means that the conditions $f_{c}^{(I X V)}=f_{c}^{(F P)}$ and $\dot{q}_{w}^{(I X V)}=\dot{q}_{w}^{(F P)}$ set the interval inside which the boundary layer thickness is respected.

The $\zeta_{f_{c}}$ and $\zeta_{\delta}$ distributions obtained at the different locations on the IXV are shown in Fig. 5.4 . Note that the $\zeta_{f_{c}}$ distribution is similar to the $\zeta_{\dot{q}_{w}}$ from Fig. 5.3 , meaning that one way of applying the methodology can be chosen over the other, depending on which parameter better fulfills the objectives of the extrapolation. Looking at the $\zeta_{\delta}$ distribution, one notices a peak in the discrepancies. This could mean that, although the heat flux and the outer edge values are the same for both flat plate and IXV simulations, the boundary layer profiles are different. This is addressed in Sec. 5.3 .

Each extrapolation option discussed above leads to different results regarding the length of the flat plate. Results of $L_{F P}$ are reported in Table 5.8. It is seen that both $f_{c}^{(I X V)}=f_{c}^{(F P)}$ and $\dot{q}_{w}^{(I X V)}=\dot{q}_{w}^{(F P)}$ set a minimum and a maximum for $L_{F P}$, respectively, with the approximate $L_{F P}$ $=\xi^{(I X V)} / \rho_{e} \mu_{e} u_{e}$ option being in-between. This fact defines a range of $L_{F P}$ inside which the extrapolation methodology can be applied. It would also explain why the heat flux discrepancies 
Table 5.7.: Relative difference of the output variables with respect the IXV, if $\dot{q}_{w}^{(I X V)}=\dot{q}_{w}^{(F P)}$

\begin{tabular}{lccccc}
\hline \hline & $(\mathrm{a})$ & $(\mathrm{b})$ & $(\mathrm{c})$ & $(\mathrm{d})$ & $(\mathrm{e})$ \\
\hline$\zeta_{f_{c}}, \%$ & -8.94 & -17.78 & -17.94 & -16.19 & -16.40 \\
$\zeta_{\delta}, \%$ & 2.63 & 11.42 & 7.03 & 4.98 & 2.25 \\
\hline \hline
\end{tabular}

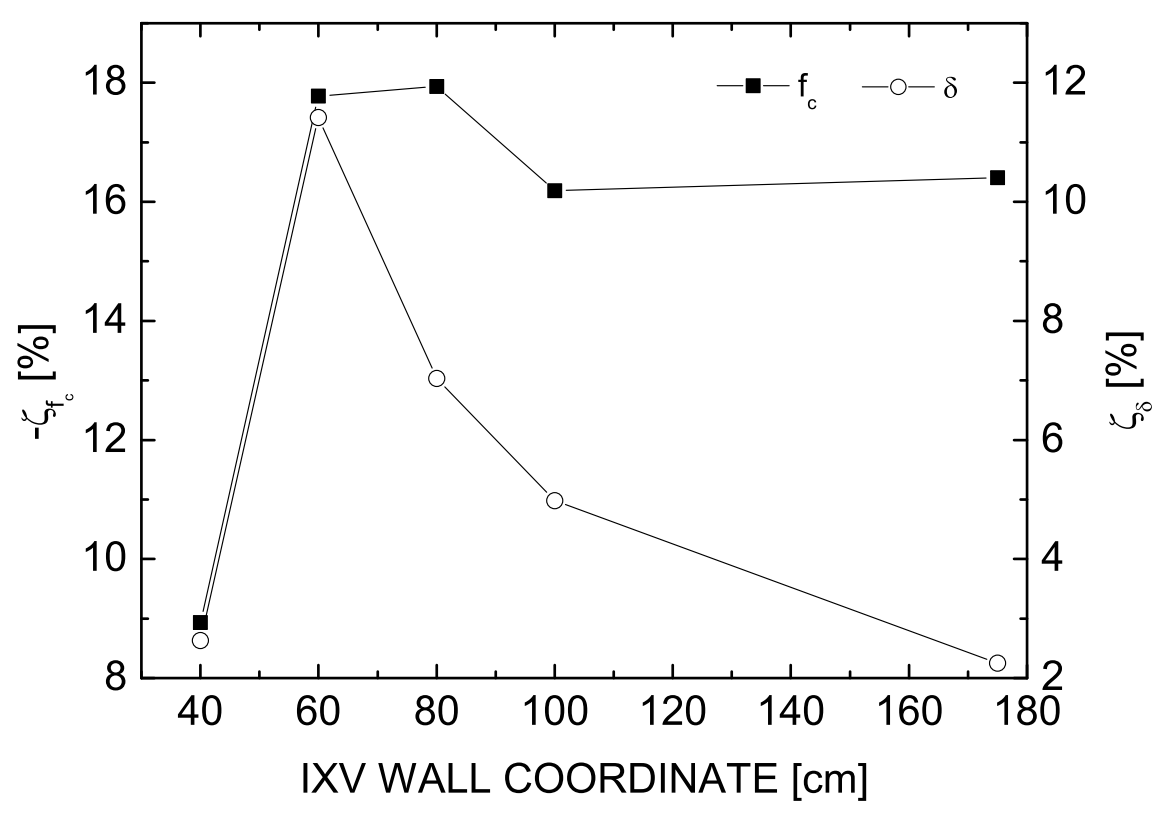

Figure 5.4.: Relative differences of flight condition factor and thickness with respect to the IXV, under the same $\dot{q}_{w}$ at different extrapolating locations.

reported in Table 5.6 are lower than those in Table 5.5. Indeed, the overestimation of the heat flux observed when $f_{c}^{(I X V)}=f_{c}^{(F P)}$ is reduced by applying the extrapolation to a longer flat plate.

\subsubsection{Scale effect}

Although the off-stagnation point boundary layer on a flying body can be duplicated on a flat plate, putting in practice this extrapolation methodology in a wind tunnel might not be always feasible given the size of the flat plates reported in Table 5.8. For instance, with the flat plate available in the VKI-Plasmatron facility measuring $24 \mathrm{~cm}$, and for the option $\dot{q}_{w}^{(I X V)}=\dot{q}_{w}^{(F P)}$, the extrapolation can not be directly applied $40 \mathrm{~cm}$ downstream from the stagnation point of the IXV. This is the reason why a scaling procedure should be applied in order to reproduce the flight boundary layer on ground.

To proceed, the inlet velocities are calculated with Eq. 5.5 according to a reference flat plate model of $20 \mathrm{~cm}$. This reference length is taken because it is representative of the flat plate model mentioned above, which has been extensively used in the VKI-Plasmatron [11, 106, 108, 109, 112. The scaling exercise is carried out using the $L_{F P}$ values obtained in Sec. 5.2.1 for the extrapolation 
Table 5.8.: $L_{F P}$ obtained using different extrapolating options (in $\mathrm{cm}$ ), from (a) to (e)

\begin{tabular}{lccccc}
\hline \hline Extrapolating option & $(\mathrm{a})$ & $(\mathrm{b})$ & $(\mathrm{c})$ & $(\mathrm{d})$ & $(\mathrm{e})$ \\
\hline$f_{c}^{(I X V)}=f_{c}^{(F P)}$ & 21.31 & 38.37 & 55.85 & 71.69 & 129.55 \\
$L_{F P} \approx \xi^{(I X V)} / \rho_{e} \mu_{e} u_{e}$ & 24.31 & 42.48 & 63.40 & 80.80 & 148.52 \\
$\dot{q}_{w}^{(I X V)}=\dot{q}_{w}^{(F P)}$ & 26.13 & 56.56 & 83.06 & 102.04 & 184.37 \\
\hline \hline
\end{tabular}

option $\dot{q}_{w}^{(I X V)}=\dot{q}_{w}^{(F P)}$. The same flat plate mesh is used here, and the simulations are repeated changing the inlet velocities for stations (a) to (e) as follows: $U_{\text {test }}^{(a)}=903.86 \mathrm{~m} / \mathrm{s}, U_{\text {test }}^{(b)}=483.59 \mathrm{~m} / \mathrm{s}$, $U_{\text {test }}^{(c)}=327.48 \mathrm{~m} / \mathrm{s}, U_{\text {test }}^{(d)}=269.00 \mathrm{~m} / \mathrm{s}$ and $U_{\text {test }}^{(e)}=157.57 \mathrm{~m} / \mathrm{s}$. The other input parameters are kept the same. The relative differences of $T_{w}, \delta$ and $\dot{q}_{w}$ on the reduced flat plate with respect to the IXV simulation results are shown in Table 5.9. There is a heat flux reduction of $5 \%$ or less between the IXV and the model used in a wind tunnel, which is consequence of neglecting the kinetic energy contribution to the total energy exchange in the flow during the scaling process.

Table 5.9.: Relative difference of the output variables on the flat plate model with respect the IXV results

\begin{tabular}{lccccc}
\hline \hline & $(\mathrm{a})$ & $(\mathrm{b})$ & $(\mathrm{c})$ & $(\mathrm{d})$ & $(\mathrm{e})$ \\
\hline$\zeta_{T_{w}}, \%$ & -0.45 & -1.51 & -1.18 & -1.09 & -0.92 \\
$\dot{q}_{w}, \%$ & -1.89 & -5.36 & -4.64 & -4.26 & -3.61 \\
$\zeta_{\delta}, \%$ & 2.91 & 12.97 & 7.32 & 4.71 & 0.8 \\
\hline \hline
\end{tabular}

\subsection{Comparison of the boundary layer profiles}

Although the same heating can be obtained between two flow configurations, nothing has been said about the nature of such energy exchange so far. In order to assess the quality of the extrapolation, the variation of the quantities of interest along the normal coordinate $y$ are compared. For sake of simplicity, only conditions (a) and (e) are considered here. For instance, the temperature profiles are shown in Fig. 5.5 and Fig. 5.6 for case (a) and (e), respectively. In both cases, good agreement is obtained among IXV, large flat plate and flat plate model configurations.

The velocity profiles for condition (a) are plotted in Fig. 5.7, they show how the velocity in the IXV simulation is higher than on the flat plate. This difference is the consequence of the entropy layer that develops behind the bow shock around the nose of IXV, a feature absent from the flat plate simulations. Indeed, this entropy layer increases vorticity close to the stagnation region, making the velocity gradients higher on the IXV. Note, however, that the presence of the entropy layer is not relevant to duplicate the heat flux when $f_{c}^{(I X V)}=f_{c}^{(F P)}$, as reported in Table 5.5, because of the low Eckert number at this location $(E c=0.082)$. During the analysis, it has been observed that the entropy layer is completely encompassed at (b) by the kinetic boundary layer around the vehicle, making the entropy layer not noticeable at locations further downstream. 


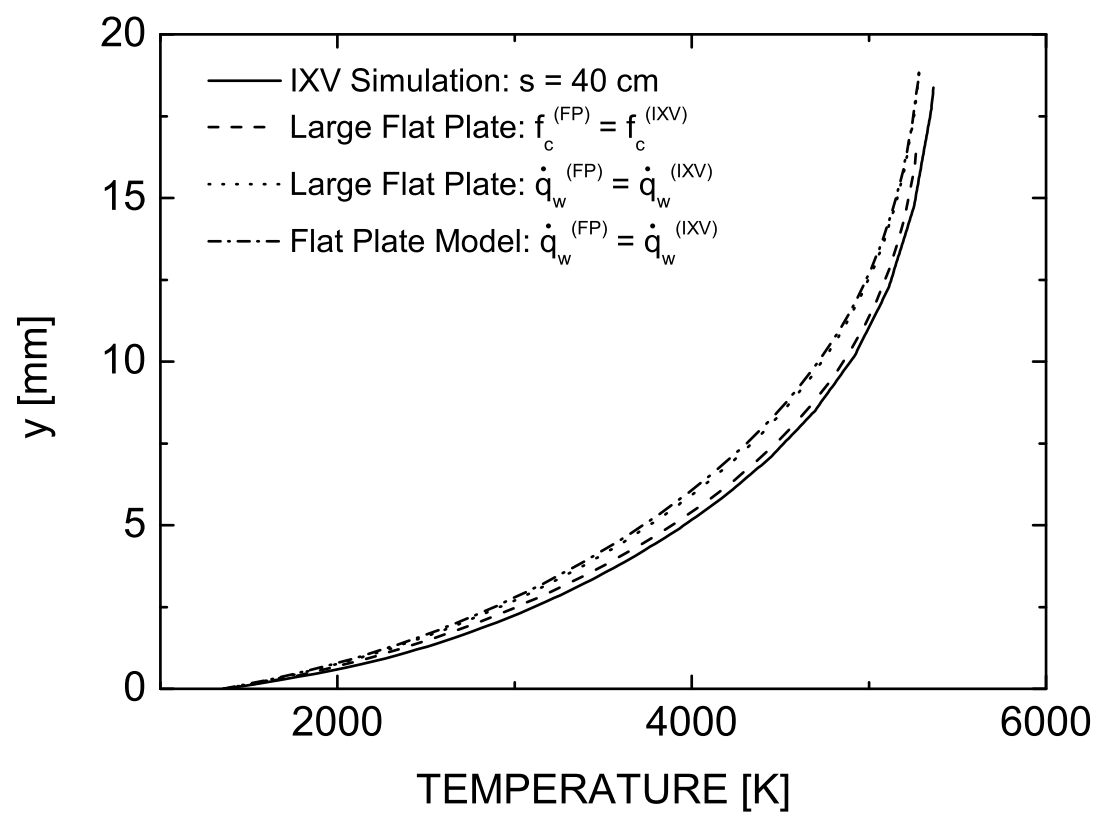

Figure 5.5.: Temperature profiles after the extrapolation: location (a).

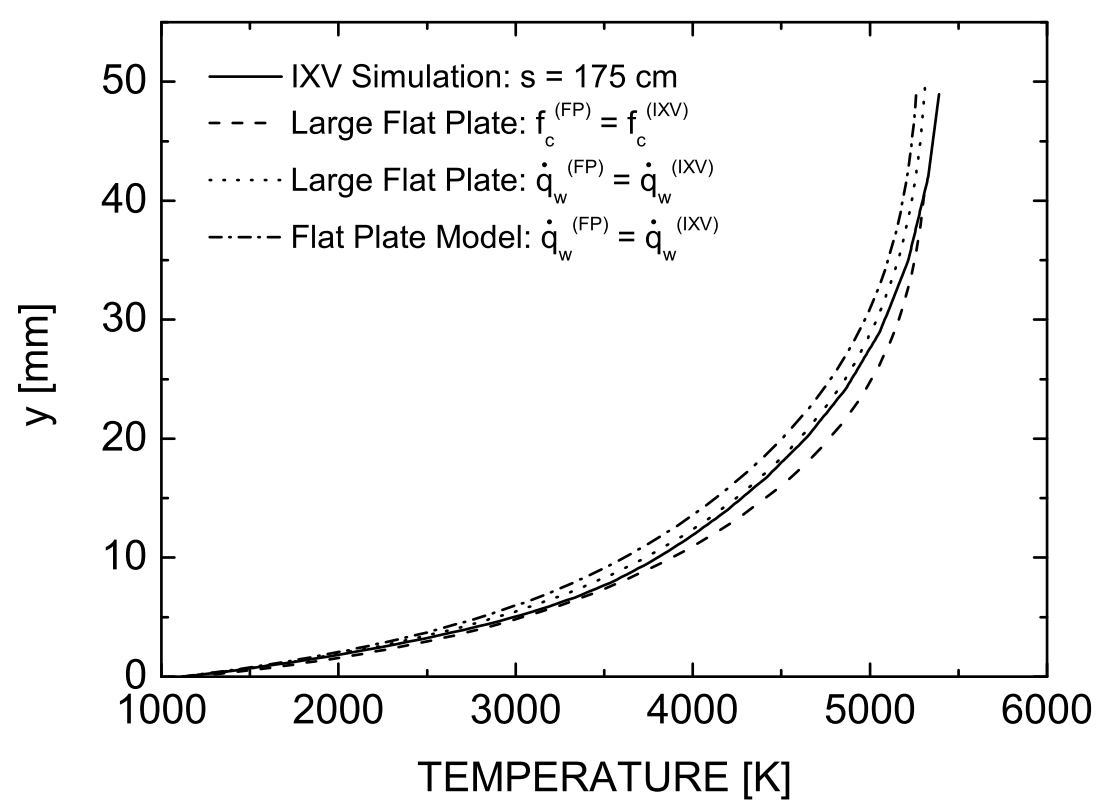

Figure 5.6.: Temperature profiles after the extrapolation: location (e).

Conversely, the velocity profiles in Fig. 5.8 at (e) show the opposite behavior, the velocity on the large flat plate for $f_{c}^{(I X V)}=f_{c}^{(F P)}$ is slightly higher than on the IXV simulation. This could be due to the fact that the velocities at the wall and at the outer edge are the same in both configurations, but the boundary layer thickness is not, which influences the gradient of velocities. The velocity profile for $\dot{q}_{w}^{(I X V)}=\dot{q}_{w}^{(F P)}$ is well reproduced on the large flat plate, leading to the conclusion that the 
boundary layer physics are better reproduced for this extrapolation option. Remark that, according to the low Eckert number assumption, the velocity for the flat plate model is never respected.

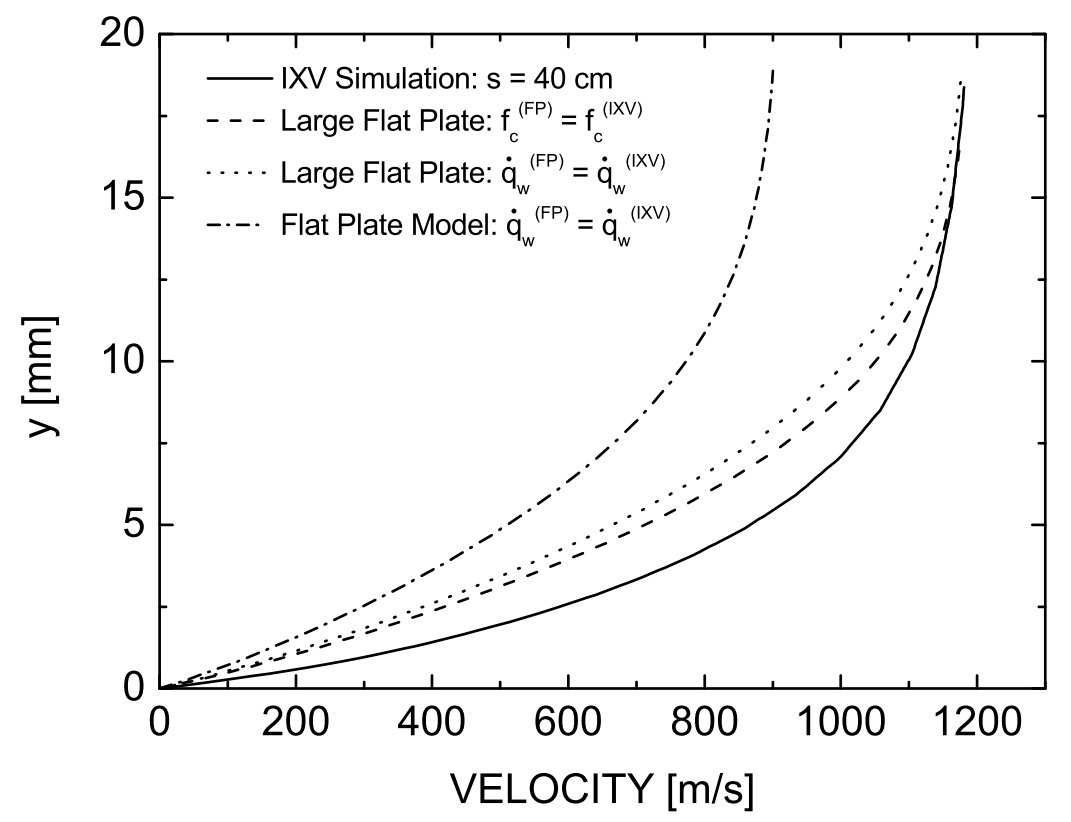

Figure 5.7.: Velocity profiles after the extrapolation: location (a).

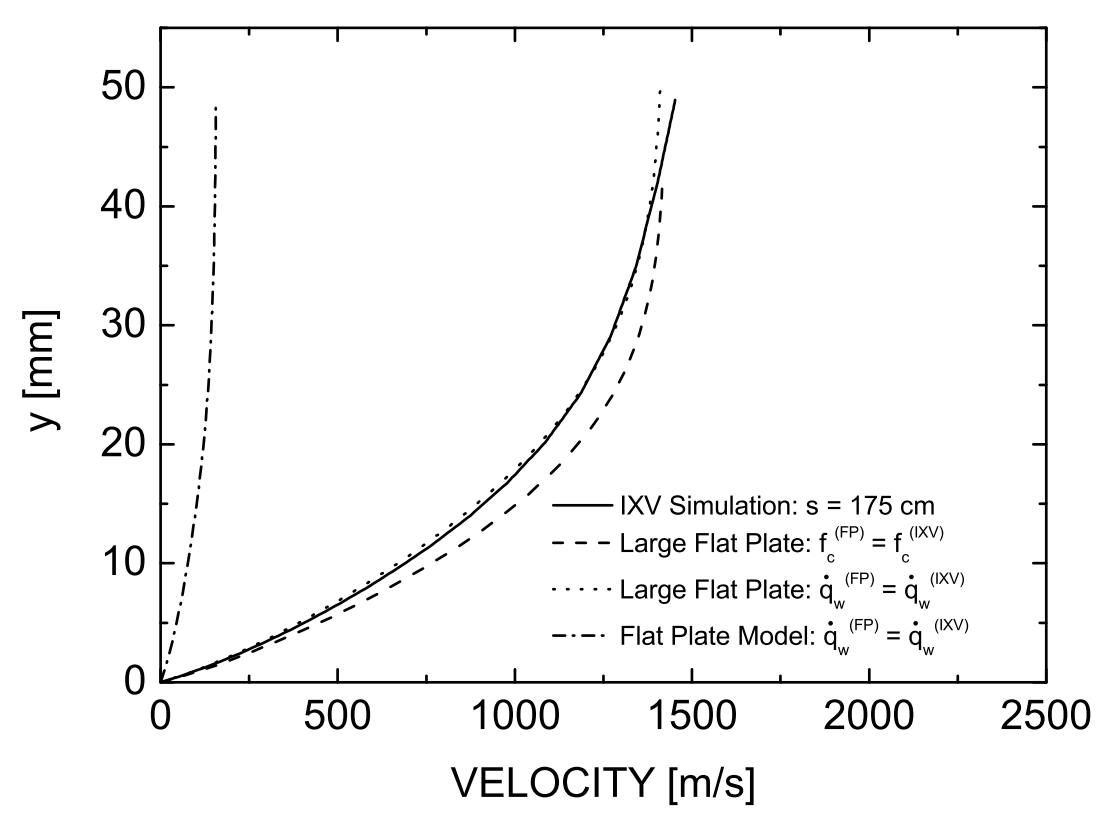

Figure 5.8.: Velocity profiles after the extrapolation: location (e).

Finally, the mass fraction profiles of N-atom are shown in Fig. 5.9 and Fig. 5.10 for the two locations. At section (a), the IXV results show a higher level of recombination than the flat plates, 
probably as an effect of the entropy layer mentioned above; while profiles match closely at (e). Again, the physics of the flow are better extrapolated setting $\dot{q}_{w}^{(I X V)}=\dot{q}_{w}^{(F P)}$ to define $L_{F P}$.

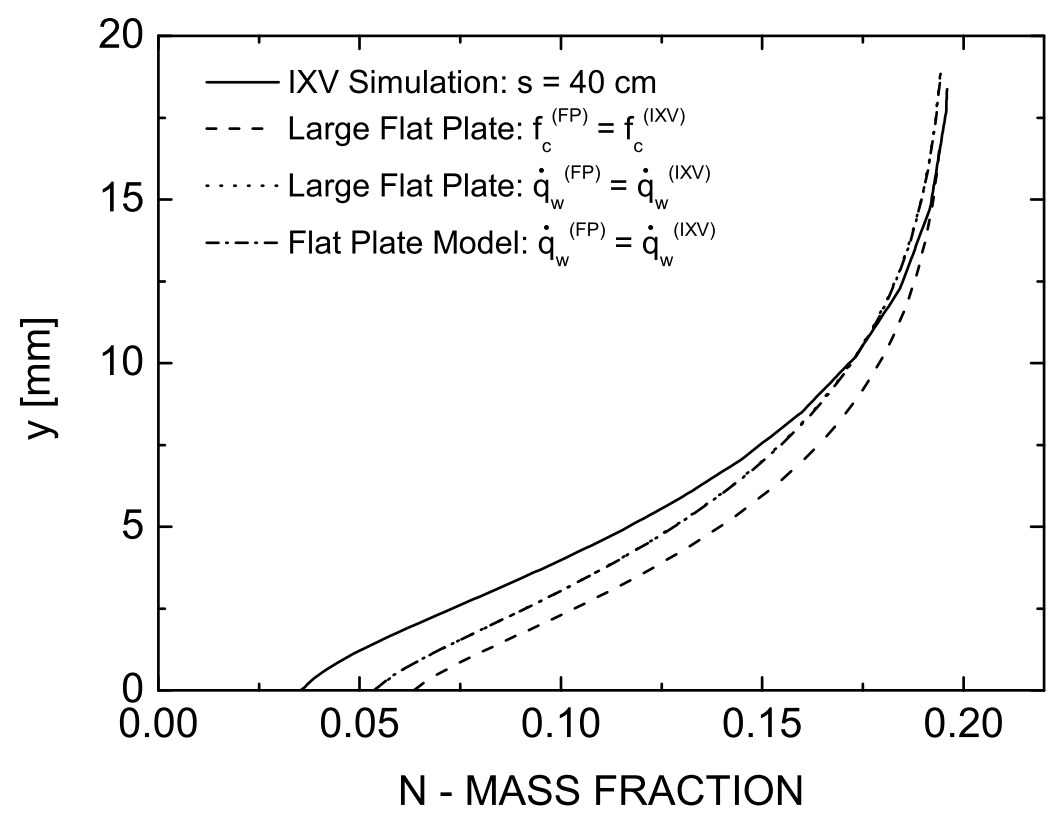

Figure 5.9.: N-atom profiles after the extrapolation: location (a).

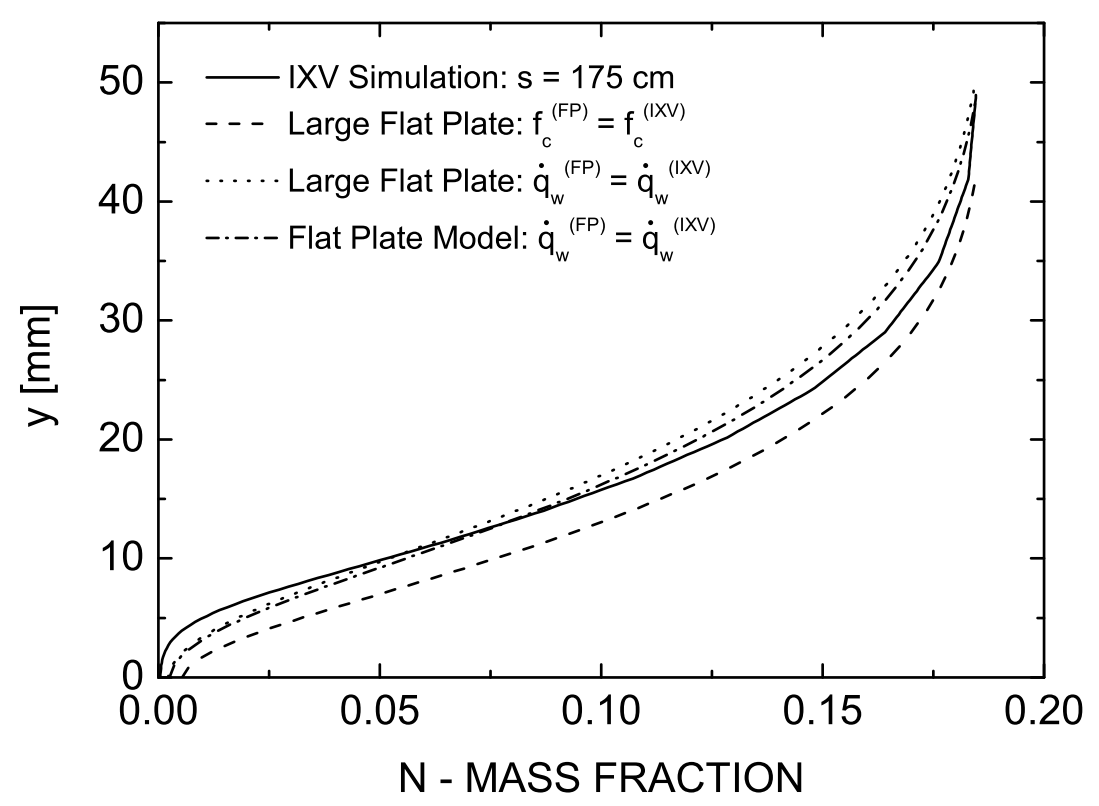

Figure 5.10.: N-atom profiles after the extrapolation: location (e). 


\subsection{Validation of the methodology}

A validation campaign for the off-stagnation point extrapolation methodology is carried out in the VKI-Plasmatron with both the flat plate model and the calorimeter described in Sec. 4.2.2. Tests being focused on the scaling effect of the extrapolation, the logic behind the experiment relies on the heat flux duplication along the calorimeter for two testing conditions, where pressure and enthalpy are the same and frees-stream velocity is varied with the gas mass flow rate $\dot{m}$ injected into the Plasmatron chamber. This should allow scaling the model by keeping the same convective time $x / U_{\text {test }}$ between plates, as explained in Sec. 5.1 .

However, some additional CFD simulations should be run before testing in order to address possible issues that might appear when applying the off-stagnation point extrapolation methodology in a plasma wind tunnel.

\subsubsection{CFD assessment for flow characterization}

The fact that the boundary layer edge properties along the plate cannot be measured in a plasma wind tunnel test forces the methodology to rely on free-stream flow characterization at the stagnation line. This means that, although edge enthalpy and velocity are required inputs, they must be estimated from the stagnation line quantities for validation. This motivates a CFD assessment to study if the stagnation line values, together with the assumption of uniform free-stream profiles, can be used as reference before applying the methodology.

This numerical assessment is carried out with the CFD ++ software using the same mesh configuration used in Sec. 4.4. Two flow aspects are taken into account: one is the appearance of discrepancies between the edge enthalpy along the plate and the stagnation enthalpy rebuilt with CERBOULA due to the jet structure of the plasma developing downstream the torch exit in the Plasmatron, and the other is that a variation in the free-stream velocity leads to the same variation at the boundary layer edge.

To assess differences in enthalpy, both temperature and velocity profiles are generated through a polynomial extrapolation from experimental data provided by Cipullo [113] (for temperature) and Baldani [114] (for velocity). The only test condition that these references have in common (16 $\mathrm{g} / \mathrm{s}, 200 \mathrm{~kW}$ and $100 \mathrm{mbar}$ ) is used to extrapolate the temperature and velocity profiles that are introduced at the inlet of the CFD simulations. A local maximum in temperature and velocity is enforced at the centerline of the jet and the extrapolation results are shown in Fig. 5.11 and Fig. 5.12, respectively. Note that, unlike the procedure followed in Sec. 4.4, the inlet profiles are not based on any ICP computation, but on actual experimental data. Remark also that the lack of measurements far from the symmetry axis makes the profile extrapolation less reliable at the outer region of the plasma jet. However, this is not considered critical to draw conclusions on the flow topology close to the flat plate model. Species profiles are computed with Mutation ++ [71] under the assumption of LTE. The wall is assumed full-catalytic and at $350 \mathrm{~K}$. 


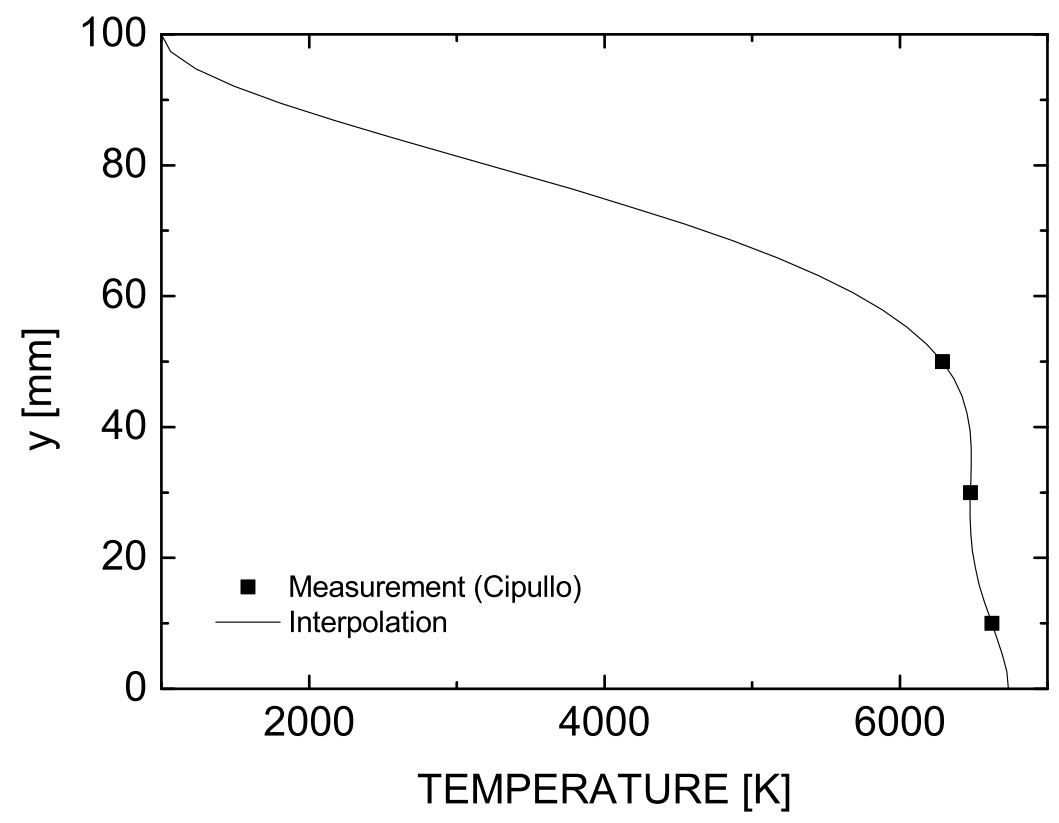

Figure 5.11.: Interpolated inlet temperature profile from Plasmatron measurements [113].

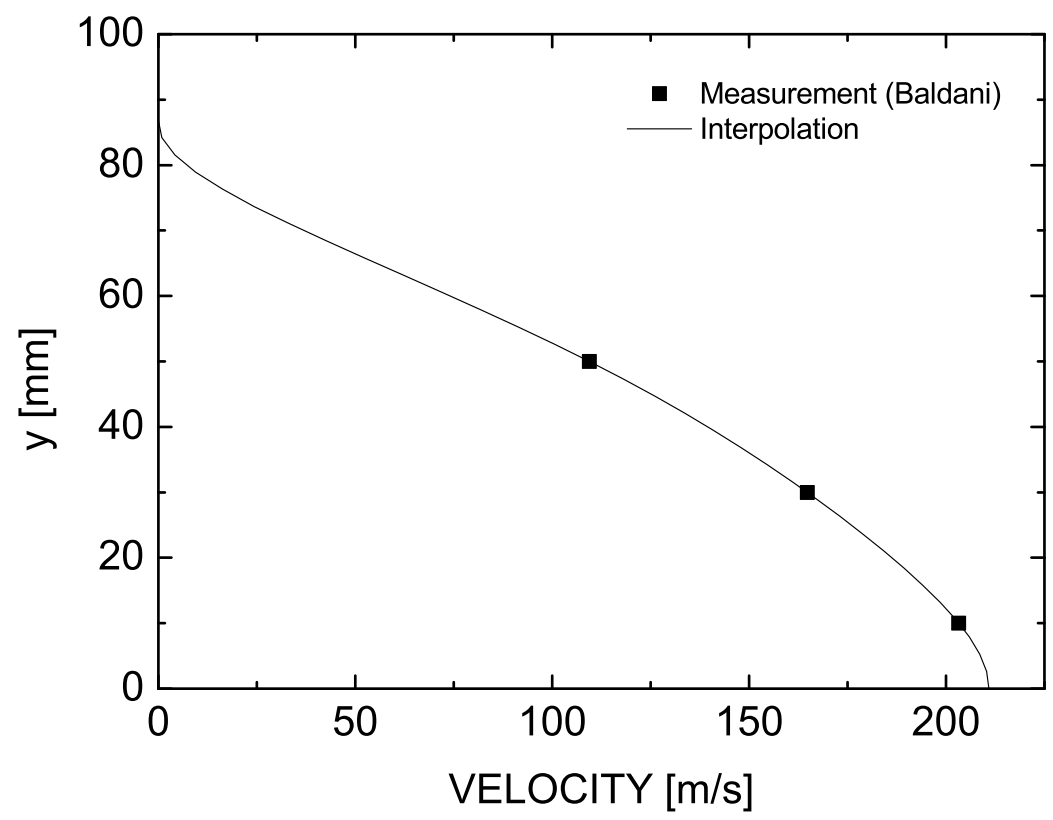

Figure 5.12.: Interpolated inlet velocity profile from Plasmatron measurements [114].

Static enthalpy results at 10 and $20 \mathrm{~cm}$ from the leading edge are plotted in Fig. 5.13 and Fig. 5.14 , respectively. They are also compared to simulations with uniform inlets. From these plots, one can conclude that the free-stream enthalpy on the stagnation line and the boundary layer outer edge are different due to the jet profile. This means that, while enthalpy at the stagnation point can be determined with CERBOULA after a Plasmatron test, its value at off-stagnation point will remain 
unknown for the experimental validation of the extrapolation methodology. Note that this fact can bring issues when applying the extrapolation methodology between the flying body and the flat plate model in future Plasmatron test campaigns, because matching outer edge enthalpies between flight and ground in a shear flow configuration will be difficult to achieve experimentally. However, for the case at hand, the enthalpy at the outer edge of the boundary layer around the flat plate model will be assumed to be equal for two test conditions as long as the enthalpy determined with CERBOULA at the stagnation point remains the same.

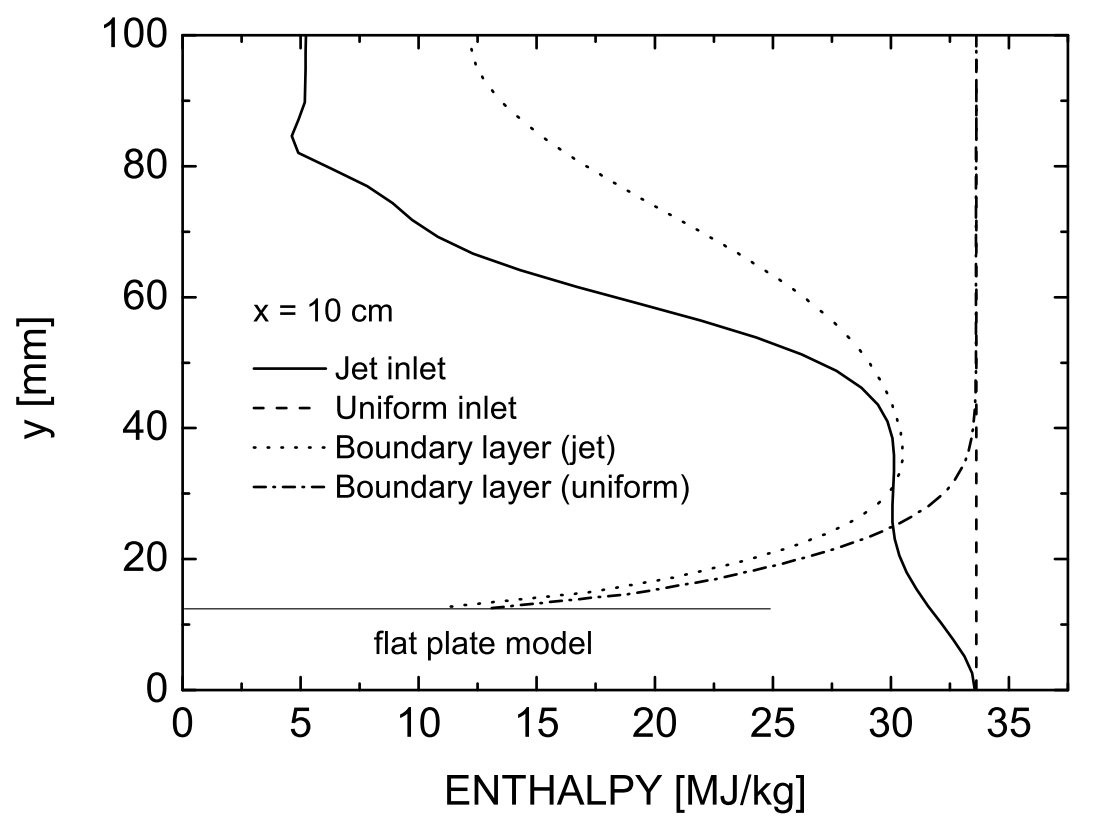

Figure 5.13.: Enthalpy profiles assessment at $10 \mathrm{~cm}$ from the leading edge.

To assess how the variation in free-stream velocity affects the outer edge of the model's boundary layer, two additional simulations are carried out under uniform inlets, with $T_{\infty}=6735 \mathrm{~K}, P_{\infty}=100$ mbar and LTE imposed for the species mass fractions. The only difference between simulations being the inlet velocity, one is the double of the other: $U_{\infty}=210.90 \mathrm{~m} / \mathrm{s}$ and $U_{\infty}=105.45 \mathrm{~m} / \mathrm{s}$. Again, the wall is considered isothermal at $350 \mathrm{~K}$ and full-catalytic. Simulation results are shown in Fig. 5.15 and Fig. 5.16 at both 10 and $20 \mathrm{~cm}$ from the leading edge of the flat plate. It is observed that the boundary layer edge velocity at different locations on the model is halved if differences in free-stream velocities are related by a factor of $1 / 2$. This means that any velocity variation on the Plasmatron should lead to the same variation at the edge of the boundary layer along the plate, making the scaling of the boundary layer on a flat plate experimentally possible.

\subsubsection{Test campaign and results}

One requirement to validate the off-stagnation point extrapolation methodology is to modify the testing velocity while keeping the same enthalpy and pressure conditions, which leads to the same gas composition under LTE. This means flow velocity cannot be adjusted through pressure variations because pressure plays a significant role on the species composition of the gas, as shown in Ref. [105]. 
Therefore, the only strategy to vary the flow speed is by changing the mass flow of gas $\dot{m}$ introduced in the test chamber. As most Plasmatron tests are carried out at $16 \mathrm{~g} / \mathrm{s}$, new measurements taken at $8 \mathrm{~g} / \mathrm{s}$ are provided below.

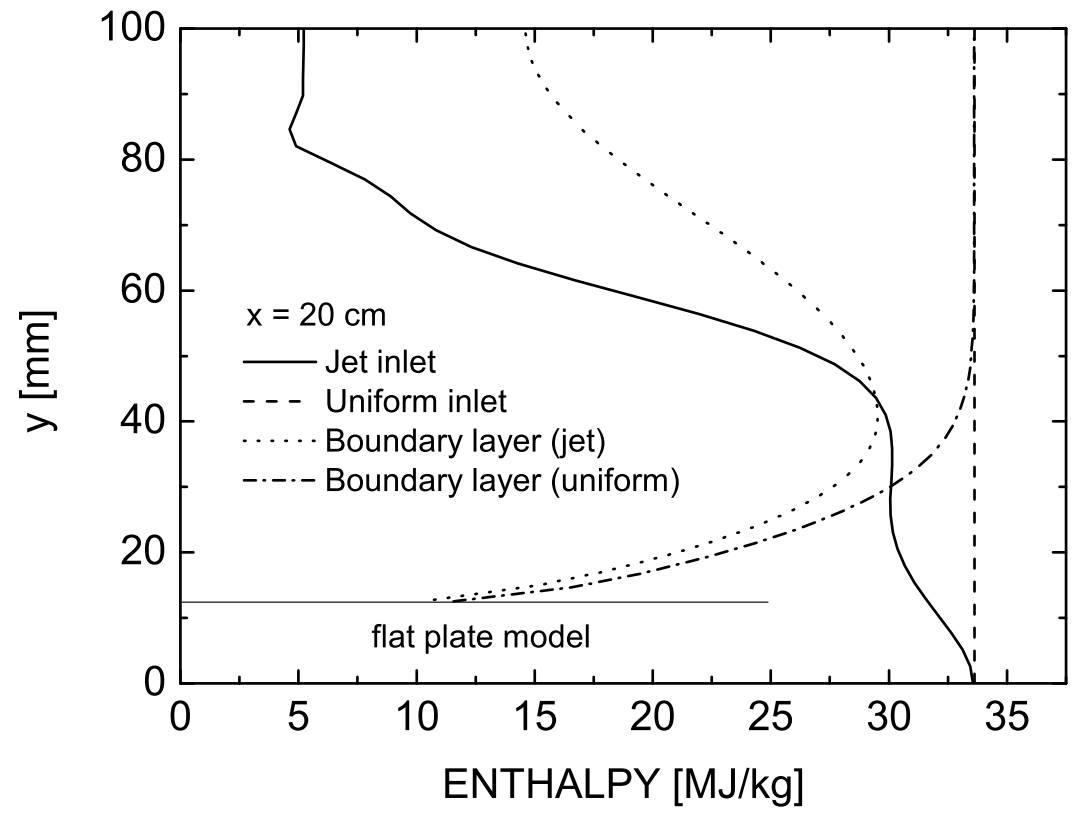

Figure 5.14.: Enthalpy profiles assessment at $20 \mathrm{~cm}$ from the leading edge.

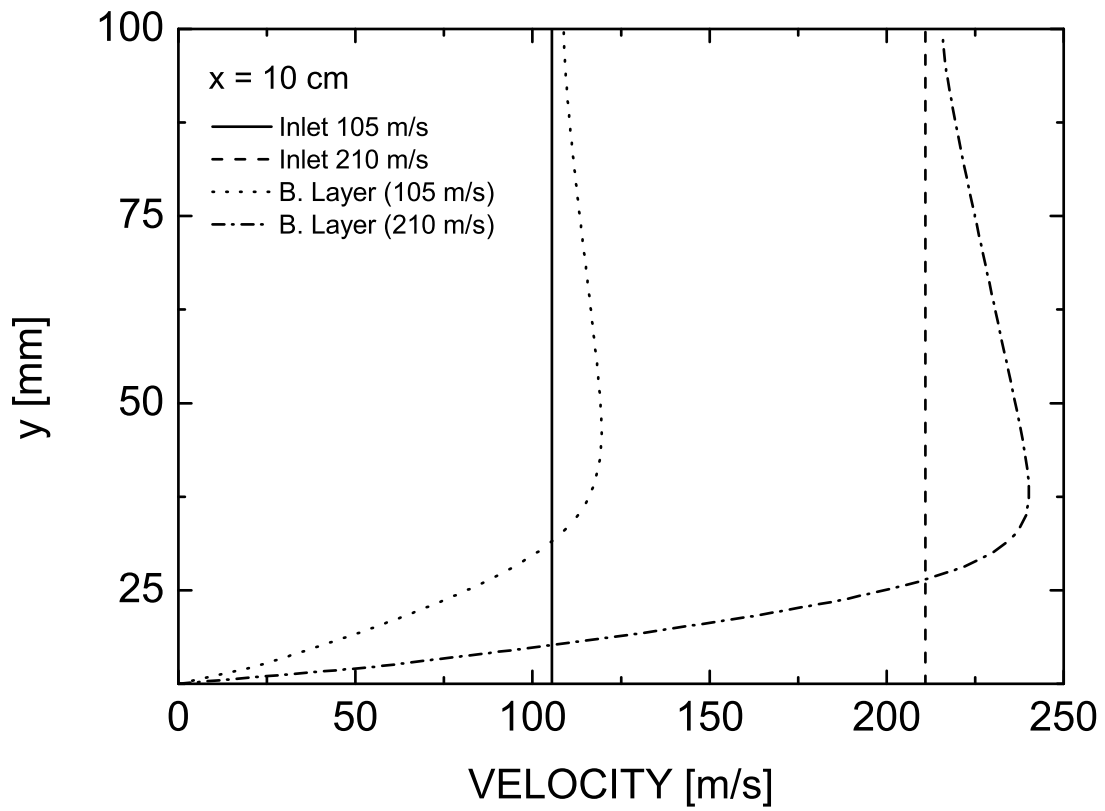

Figure 5.15.: Velocity variation assessment at $10 \mathrm{~cm}$ from the leading edge. 


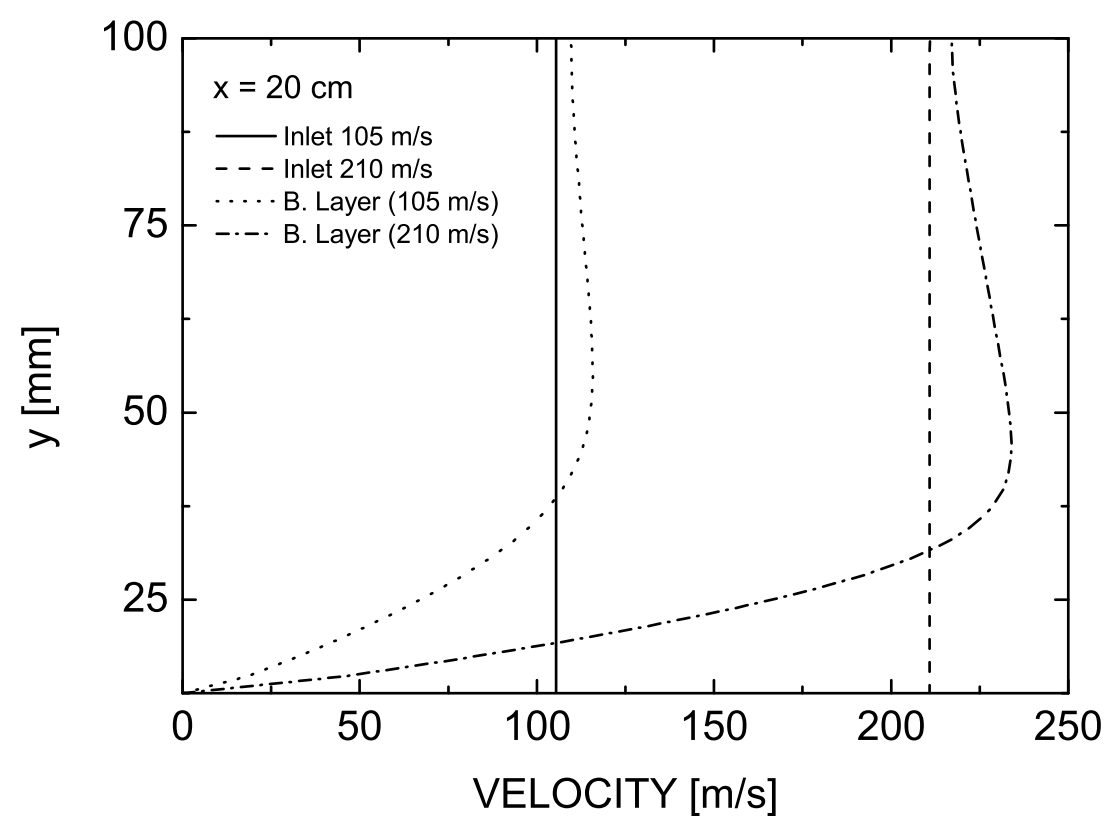

Figure 5.16.: Velocity variation assessment at $20 \mathrm{~cm}$ from the leading edge.

A calibration campaign is carried out in the Plasmatron before testing on the flat plate. The purpose of such calibration is to define the two target heat flux conditions on the Standard probe so that the same free-stream enthalpy is obtained under 16 and $8 \mathrm{~g} / \mathrm{s}$. This calibration is thoroughly explained in Ref. [115] and consists on rebuilding the enthalpy at the boundary layer outer edge with CERBOULA for different heat flux measurements on the Standard probe. The test matrix of Table 5.10 is selected for the extrapolation methodology validation at off-stagnation point. Note that, although a wide pressure range has been used in previous campaigns during this thesis, testing at $8 \mathrm{~g} / \mathrm{s}$ under high pressure conditions is not easily achieved at the Plasmatron facility. In addition, testing with the flat plate at high power levels increases the probability of water inside the calorimeter pipe approaching boiling temperatures at the last thermocouple, endangering the measurement reliability. This is an undesirable situation that should be avoided whenever possible. In consequence, only tests at 15 mbar and low powers are considered here.

Table 5.10.: Test matrix for off-stagnation point methodology validation

\begin{tabular}{lrrr}
\hline \hline Test & $\begin{array}{r}\dot{m} \\
\mathrm{~g} / \mathrm{s}\end{array}$ & $\begin{array}{r}P_{s} \\
\mathrm{~Pa}\end{array}$ & $\begin{array}{r}\dot{q}_{c w}^{(r e f)} \\
\mathrm{kW} / \mathrm{m}^{2}\end{array}$ \\
\hline $\mathrm{a}$ & 16 & 1500 & 600 \\
$\mathrm{~b}$ & 8 & 1500 & 500 \\
\hline \hline
\end{tabular}

The test configuration is the same as that shown in Fig. 4.21. Probes are aligned with the plasma torch and located $445 \mathrm{~mm}$ from the torch exit. The Standard heat flux probe is injected first and power is increased until the target conditions are reached. Then, the dynamic pressure 
probe is introduced for free-stream characterization with CERBOULA. Finally, the flat plate model that holds the linear calorimeter is exposed to the plasma and the temperature distribution along the 9 type-E thermocouples is measured. Water mass flow inside the pipe is kept constant with a rotameter at $5.1 \mathrm{~g} / \mathrm{s}$ during plasma exposure. Data from all thermocouples in the system and from the differential pressure transducer are constantly recorded by the data acquisition system at $1 \mathrm{~Hz}$. Measured quantities and rebuilt free-stream conditions with CERBOULA are provided in Table 5.11

Time-averaged temperature distributions along the linear calorimeter are provided in Table 5.12 at both (a) 16 and (b) $8 \mathrm{~g} / \mathrm{s}$, and shown in Fig. 5.17. As before, a logarithmic interpolation of the form $T(x)=A \ln (x)+B$ is carried out among the temperature measurements on the flat plate, so that the heat flux can be derived from Eq. 4.16. The fitting parameters are written in Table 5.13 and the heat flux distributions are plotted in Fig. 5.18. A $\pm 10 \%$ of uncertainty is assumed at the measuring points. Remark that a problem is detected on the last thermocouple, which reads a significant temperature jump with respect to the previous one. The cause of such behavior is not known so far because it is not observed on other testing campaigns. One possible explanation could be that the $9^{\text {th }}$ thermocouple is in contact with the cooling system of the plate holder, the temperature of which can be significantly higher because it is located at the hot end of the water circuit.

Table 5.11.: Stagnation point measurements and rebuilt free-stream conditions

\begin{tabular}{lrrrrr}
\hline \hline Test & $\begin{array}{r}P W \\
\mathrm{~kW}\end{array}$ & $\begin{array}{r}\dot{q}_{c w}^{(r e f)} \\
\mathrm{kW} / \mathrm{m}^{2}\end{array}$ & $\begin{array}{r}P_{d y n} \\
\mathrm{~Pa}\end{array}$ & $\begin{array}{r}h_{\infty} \\
\mathrm{MJ} / \mathrm{kg}\end{array}$ & $\begin{array}{r}U_{\infty} \\
\mathrm{m} / \mathrm{s}\end{array}$ \\
\hline $\mathrm{a}$ & 195 & 596.59 & 336.24 & 11.73 & 839.93 \\
$\mathrm{~b}$ & 153 & 498.25 & 157.01 & 11.61 & 572.21 \\
\hline \hline
\end{tabular}

Table 5.12.: Thermocouple locations from the leading edge and their time-averaged temperature measurements along the linear calorimeter for extrapolation methodology validation at offstagnation point

\begin{tabular}{rrrrrrrr}
\hline \hline$i$ & $\begin{array}{r}x_{i} \\
{[\mathrm{~mm}]}\end{array}$ & $\begin{array}{r}T_{i}^{(a)} \\
{\left[{ }^{\circ} \mathrm{C}\right]}\end{array}$ & $\begin{array}{c}T_{i}^{(b)} \\
{\left[{ }^{\circ} \mathrm{C}\right]}\end{array}$ & $i$ & $\begin{array}{r}x_{i} \\
{[\mathrm{~mm}]}\end{array}$ & $\begin{array}{r}T_{i}^{(a)} \\
{\left[{ }^{(a} \mathrm{C}\right]}\end{array}$ & $\begin{array}{r}T_{i}^{(b)} \\
{\left[{ }^{\circ} \mathrm{C}\right]}\end{array}$ \\
\hline 1 & 49 & 24.12 & 21.25 & 6 & 165 & 59.80 & 45.43 \\
2 & 62 & 35.35 & 28.89 & 7 & 190 & 63.91 & 48.21 \\
3 & 88 & 43.56 & 34.69 & 8 & 216 & 68.04 & 50.81 \\
4 & 113 & 52.57 & 40.38 & 9 & 229 & 88.52 & 66.41 \\
5 & 139 & 55.94 & 42.54 & & & & \\
\hline \hline
\end{tabular}


Table 5.13.: Fitting coefficients for $T(x)=A \ln (x)+B$

\begin{tabular}{lrrr}
\hline \hline Test & $A$ & $B$ & $\mathrm{R}^{2}$ \\
\hline $\mathrm{a}$ & 27.952 & -81.967 & 0.9882 \\
$\mathrm{~b}$ & 18.806 & -50.037 & 0.9879 \\
\hline \hline
\end{tabular}

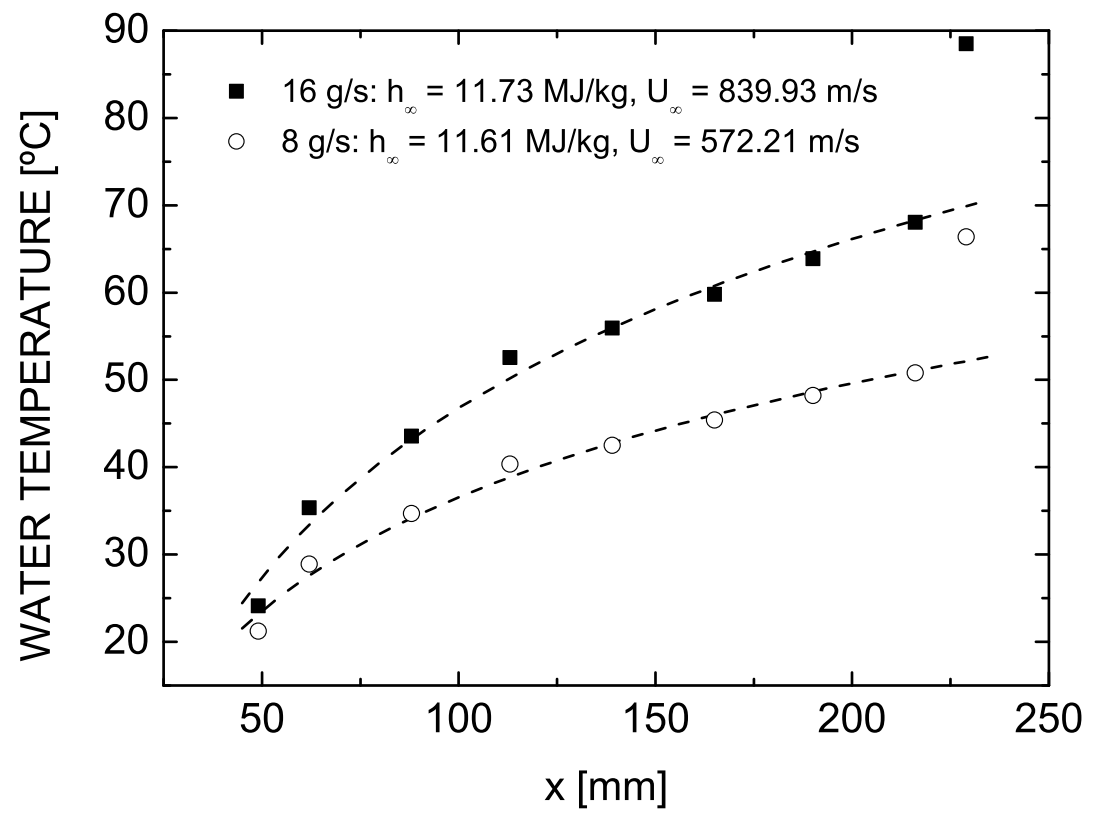

Figure 5.17.: Temperature measurements along the flat plate calorimeter.

The boundary layer can be scaled around a flat plate model if the same heat flux distribution is obtained along the convective time $x / U_{\infty}$. This would make all the parameters in the off-stagnation point heat flux equation (see Eq. 5.3 the same and, in consequence, the boundary layer would be duplicated between the two testing conditions. The convergence of the heat flux distributions at 16 and $8 \mathrm{~g} / \mathrm{s}$ is obtained and shown in Fig. 5.19, validating the off-stagnation point extrapolation methodology explained and applied along this chapter.

The relation between velocities can be further used to estimate the resulting scaling factor between the two flat plate models. For the test condition above, this relation is 0.68 , meaning that the same boundary layer evolution is obtained over a model that is $32 \%$ shorter. This result enables new Plasmatron testing capabilities for the development of flight to ground extrapolation techniques. 


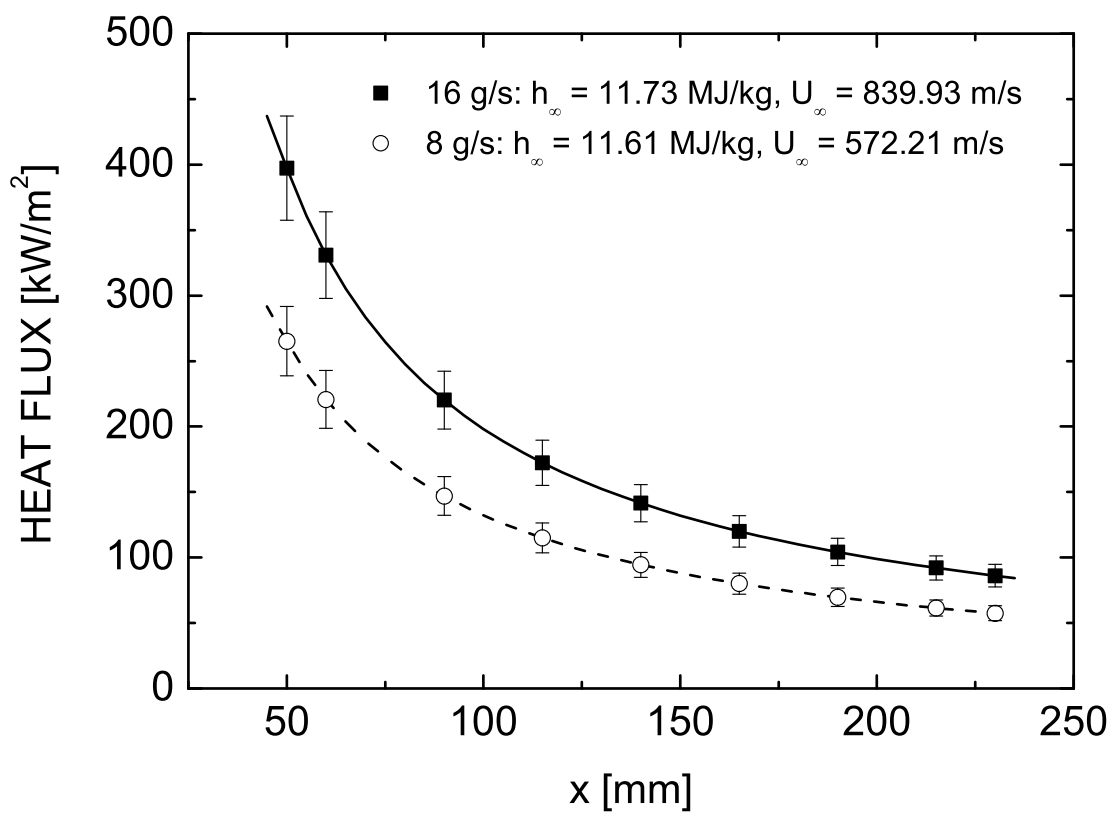

Figure 5.18.: Heat flux distributions along the flat plate calorimeter.

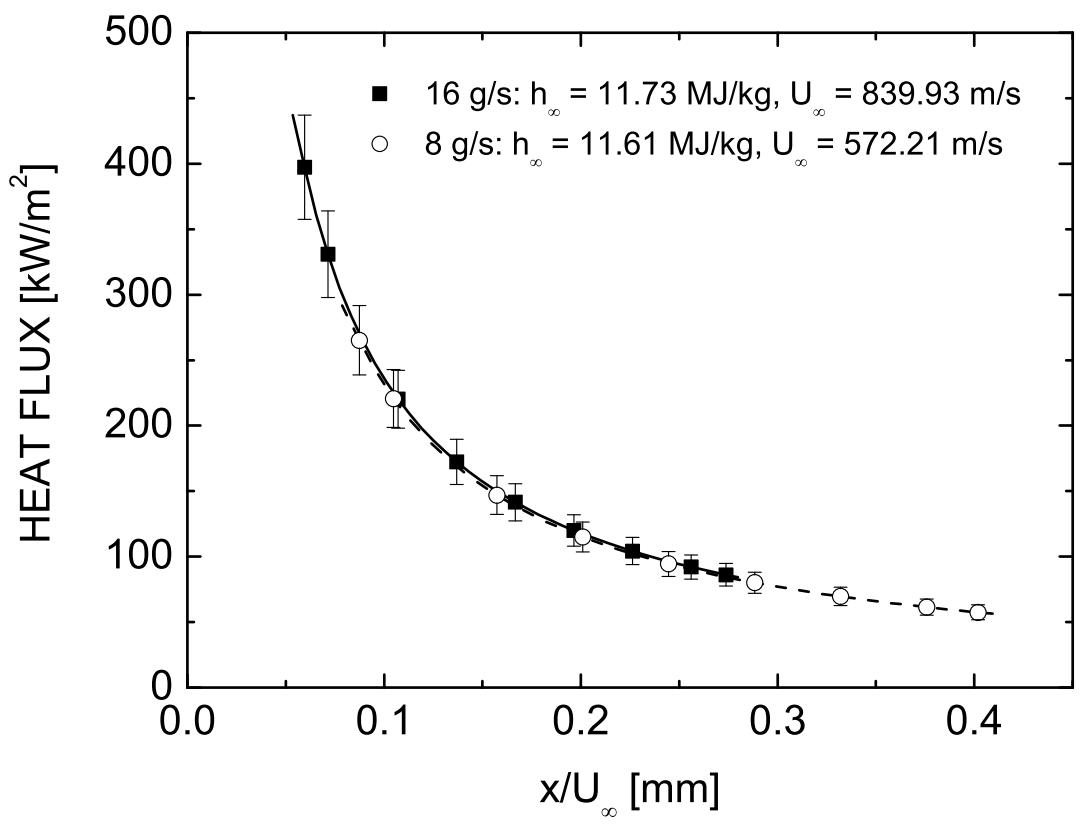

Figure 5.19.: Heat flux distributions along convective times $x / U_{\infty}$. 


\section{Chapter 6.}

\section{The flight catalysis experiment}

On February 11 ${ }^{\text {th }}, 2015$ the IXV was launched into space on board of a VV04 VEGA rocket from French Guiana. After final stage separation, it began a suborbital flight with a maximum altitude of $412 \mathrm{~km}$, while crossing checkpoints in Libreville (Gabon) and Malindi (Kenya). A scheme of the full IXV mission trajectory can be seen in Fig. 6.1. After a successful atmospheric re-entry, the IXV safely splashed-down on the Pacific Ocean, delivering invaluable flight data that will allow advances in aerothermodynamics and the future development of operational re-entry systems, such as GNC technologies and new hot structure architecture designs.

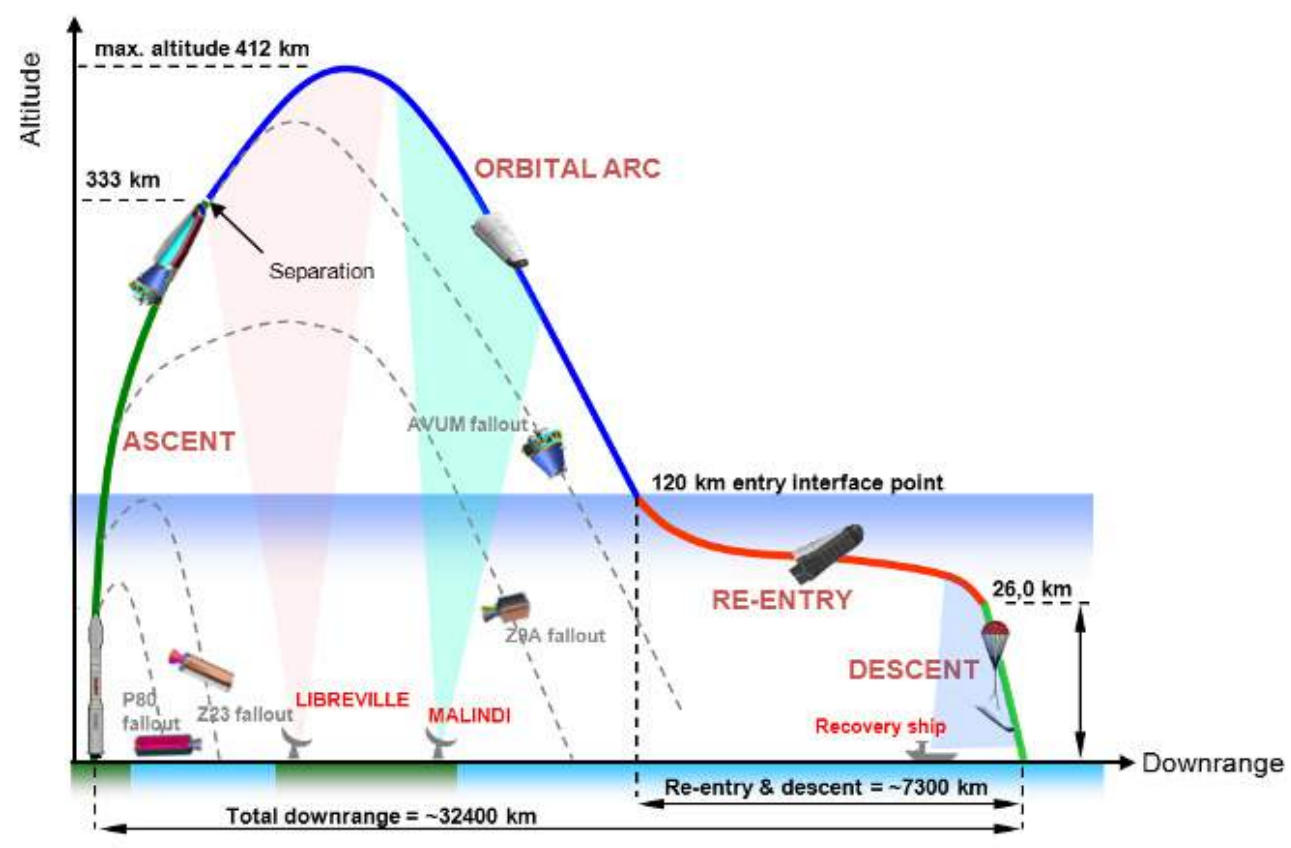

Figure 6.1.: The IXV mission flight path and stages.

The success of the IXV mission demonstrates the maturity of the European space industry in designing, qualifying and flying ceramic-based TPS. Designed by the VKI, CATE was flown integrated on the windward TPS of IXV. In harmony with the experimental nature of the mission, CATE was conceived to perform in-flight testing of GSI phenomena at the surface of a TPS.

The main motivation behind CATE is the in-flight observation of catalytic phenomena. This is achieved through the integration of a high-catalytic patch over the low-catalytic baseline TPS material of IXV. The relevance of the experiment lies on how to observe a phenomenon critical for re-entry (i.e. augmenting the exothermic heating) without endangering the safety and integrity 
of the vehicle. The Plasmatron testing capabilities at flow conditions similar to re-entry, together with thermo-mechanical simulations and microscopic assessment of the patch coating quality [116], provided enough confidence on the reliability of the $\mathrm{C} / \mathrm{SiC}-\mathrm{Mullite}$ interface for the objective of CATE and its suitability for the IXV flight [11.

CATE consists of two rectangular high-catalytic coatings deposited over the baseline $\mathrm{C} / \mathrm{SiC}$ TPS material of the windward side of IXV. The coatings were applied on the off-stagnation region, over the third and fifth TPS panels (commonly referred to as shingles). The coating was applied at the Laboratoire d'Etudes et des Recherches sur les Matériaux, les Procédés et les Surfaces (LERMPS) of UTBM-IRTES (Montbéliard, France).

The experiment is based on the principle that the enhanced recombination of $\mathrm{O}$ - and N-atoms promoted by the high-catalytic coating, compared to the lower catalytic material, determines an increase of the heat flux transferred to the wall, as the flow passes over the two surfaces. In order to measure the jump in heat flux, temperature measurements at the back surface of the TPS shell were carried out. A suite of 14 type-S thermocouples provided spatial resolution to detect the temperature jump due to the different catalytic properties of the materials. Temperature measurements were constantly recorded during the IXV re-entry, delivering data at different flight conditions. Documented catalytic transition flight experiments similar to CATE were conducted by Stewart et al. [117, 118] on board of the Space Shuttle Orbiter.

CATE objectives are:

- Demonstrate that the baseline C/SiC TPS material is not full-catalytic under re-entry conditions.

- Identify under what flight conditions catalysis is most relevant.

- Determine how different enthalpy conditions and dissociation levels influence the catalytic behavior of hot TPS surfaces.

- Reduce margins for TPS sizing due to catalycity, and improve the effectiveness of the overall TPS design.

- Provide real flight data to model catalytic phenomena

- Evaluate the temperature jump at the low-to-high catalytic interface under Low Earth Orbit (LEO) re-entry conditions.

- Assess the use of several materials in TPS design.

The contribution of this work is to document how the flight experiment was designed and integrated on the IXV system. An overview of the different steps followed towards the realization of the experiment is given. Preliminary flight data from CATE are also reported. Available trajectory information from flight is used to define the most appropriate testing conditions for the extrapolation activities at both stagnation and off-stagnation point configurations. Such preliminary flight data analysis is intended to set the initial steps for the post-flight activities to be developed at the VKI concerning the aerothermodynamic model validation using the Plasmatron facility. 


\subsection{CATE design}

A schematic of CATE is shown in Fig. 6.2. The baseline material for the IXV windward side TPS is the SepcarbInox ${ }^{\circledR} \mathrm{C} / \mathrm{SiC}$ from Herakles (Safran Group). This is a silicon carbide-based Ceramic Matrix Composite (CMC). Two rectangular (130x64mm) coatings were deposited over the surface of shingles 3 and 5 of the windward TPS in the vicinity of the vehicle symmetry axis. The selected material for the coating was $3 \mathrm{Al}_{2} \mathrm{O}_{3} 2 \mathrm{SiO}_{2}$ (or Mullite), a stable intermediate phase in the alumina-silica binary system. Mullite coatings have been extensively treated in literature as suitable for application on large exterior surfaces of space structures in a LEO environment, thanks to their excellent long-term high temperature stability in air, low thermal conductivity and very good thermal shock and creep resistance [119, 120]. Mullite was down-selected, during the development of CATE, due to its good chemical compatibility with the CMC substrate and to its favorable thermophysical properties, compared to other coating options. Characterization activities of the Mullite coating were performed at the Plasmatron using established methodologies [121. Similar emissivities at high temperature were measured for the coating and baseline TPS, and an order of magnitude higher catalycity was measured for the Mullite compared to $\mathrm{C} / \mathrm{SiC}$ at similar surface temperatures.

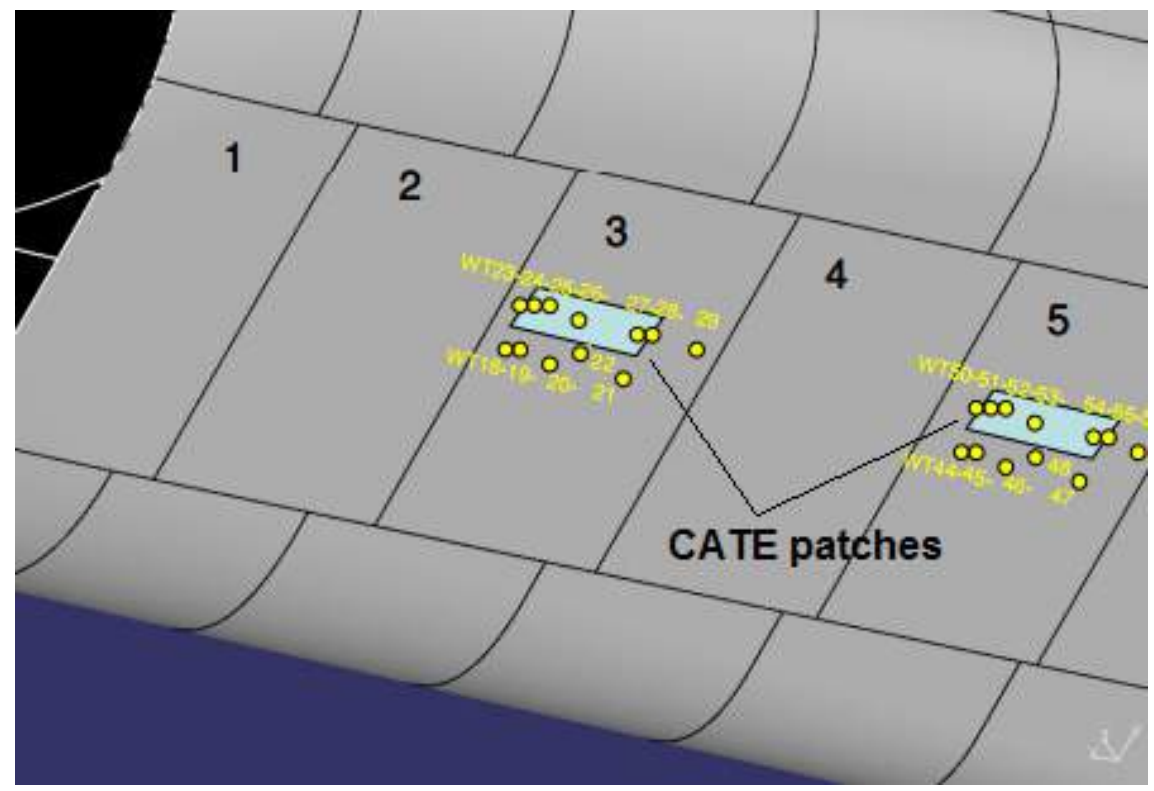

Figure 6.2.: Schematic illustration of CATE, with thermocouples. Rectangles numbered from 1 to 5 represent the five shingle panels at the windward side of IXV. The dimensions of those panels are 677 by $398 \mathrm{~mm}$.

During the IXV flight, temperature measurements were acquired at the back-face of the TPS shell, using a suite of 14 type-S thermocouples. Those were placed to ensure an optimal detection of the temperature jump on the overcoats, taking into account the constraints of the actual TPS sub-system assembly such as the presence of stiffeners, interfaces, gaps, etc. In addition, two thermocouples were placed at the centerline of the vehicle and eight more were installed symmetric to the patches' location, $39 \mathrm{~mm}$ from the IXV longitudinal symmetry plane, to measure the correspondent temperature distribution on the uncoated baseline material. The location of the thermocouples 
(also displayed in Fig. 6.2) is specified in Table 6.1 using the IXV reference coordinate system, where $x$ is the longitudinal axis of the vehicle located along its symmetry plane, with origin at the vehicle base. The low-to-high catalytic jump was located at $2807.8 \mathrm{~mm}$ in shingle 3 and at $2007.8 \mathrm{~mm}$ in shingle 5, while the high-to-low catalytic jump was at $2677.8 \mathrm{~mm}$ in shingle 3 and at $1877.8 \mathrm{~mm}$ in shingle 5. It is noted that thermocouples WT29 and WT56 were placed downstream of CATE for additional information on the flow behavior. Several other thermocouple sensors were integrated in the system to measure the temperature of IXV's windward side, e.g. the thermocouples in shingles 2 and 4 (not shown in Fig. 6.2). Although their position was not selected based on the objectives of CATE, but based on other IXV system requirements, their measurements can provide invaluable information for CATE.

Table 6.1.: Thermocouples' location and designation for CATE (with $x=0$ corresponding to the base surface of IXV). ${ }^{*}$ WT51 was reported damaged during assembly

\begin{tabular}{lcccc}
\hline \hline & $\begin{array}{c}\text { Thermocouple } \\
\text { Shingle 3 }\end{array}$ & $\begin{array}{c}x \\
\mathrm{~mm}\end{array}$ & $\begin{array}{c}\text { Thermocouples } \\
\text { Shingle 5 }\end{array}$ & $\begin{array}{c}x \\
\mathrm{~mm}\end{array}$ \\
\hline Baseline C/SiC & WT18 & 2802.8 & WT44 & 2002.8 \\
& WT19 & 2782.8 & WT45 & 1982.8 \\
& WT20 & 2742.8 & WT46 & 1942.8 \\
& WT21 & 2672.8 & WT47 & 1872.8 \\
Symmetry C/SiC & WT22 & 2735.7 & WT48 & 1935.4 \\
CATE & WT23 & 2812.8 & WT49 & 2012.8 \\
& WT24 & 2802.8 & WT50 & 2002.8 \\
& WT25 & 2782.8 & WT51 & 1982.8 \\
& WT26 & 2742.8 & WT52 & 1942.8 \\
& WT27 & 2682.8 & WT53 & 1882.8 \\
& WT28 & 2672.8 & WT54 & 1872.8 \\
WT29 & 2637.6 & WT55 & 1837.6 \\
\hline \hline
\end{tabular}

The CATE implementation process is described in the following section: from panel manufacturing to coating deposition. An assessment of the coating thickness and quality, and a description of the thermocouples' attachment strategy are also provided.

\subsection{CATE implementation}

Both the nose and the windward side assemblies of IXV were designed and developed by SafranHerakles (Bordeaux, France) on the basis of previous TPS programs. A schematic of the TPS assembly concept is shown in Fig. 6.3 for a flat shingle panel. The rigid surface that constitutes the aerodynamic shell of the vehicle exposed to the plasma flow was made of a rigid, highly refractory SepcarbInox ${ }^{\circledR}$ L6 CMC material. This hot surface was supported by the vehicle inner structure, and a lightweight, flexible insulation was installed beneath the hot surface to prevent the overheating of the internal structure and onboard sub-systems. Materials and thicknesses of the inner insulation are specified in Table 6.2, ordered from outermost to innermost layer. Remark that these materials were wrapped in Kapton foil. 


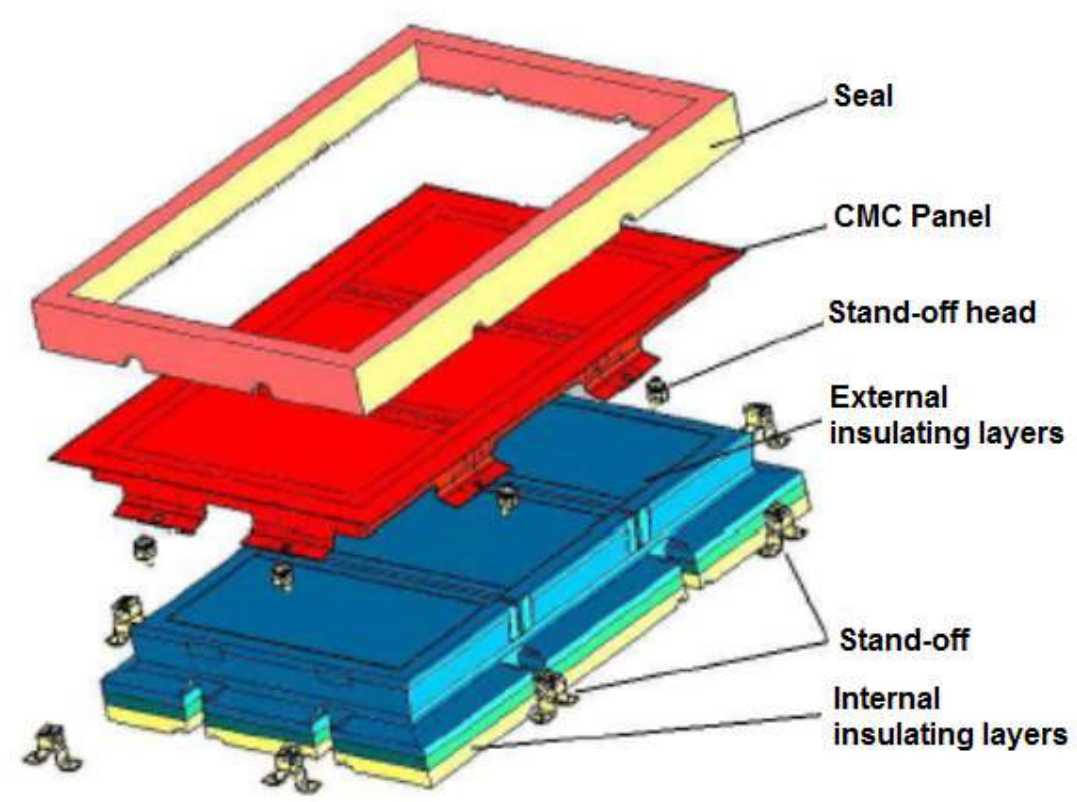

Figure 6.3.: Typical SepcarbInox ${ }^{\circledR}$ CMC TPS assembly design.

Table 6.2.: Internal insulating layers on the TPS of IXV

\begin{tabular}{lr}
\hline \hline Material & $\begin{array}{r}\text { Thickness } \\
\mathrm{mm}\end{array}$ \\
\hline Zircar AB & 81 \\
AEROGUARD $^{\circledR}$ & HD 220 \\
Pyrogel & 12 \\
\hline \hline
\end{tabular}

The $\mathrm{C} / \mathrm{SiC}$ panels were assembled onto the vehicle's cold structure by means of semi-flexible metallic attachments. The stiffness of these attachments had been adjusted to limit stresses resulting from the thermal expansion of the panels at high temperatures. These attachment devices were also equipped with insulating ceramic washers in order to further reduce the heat transfer. Finally, a fibrous seal was placed around each panel to prevent hot gases from entering through the gap between two adjacent panels.

\subsection{1. $\mathrm{C} / \mathrm{SiC}$ panel manufacturing}

The SepcarbInox ${ }^{\circledR}$ L6 C/SiC material technology is based on a delamination-resistant stacked weaving called Guipex ${ }^{\circledR}$, enabling the manufacture of monolithic CMC shells. This carbon fiber preform of the shingle was impregnated with a high-carbon content resin and laid in a mold that provided the desired shape (prepreg). The stiffeners were also added at this stage and assembled using a sewing technique involving a carbon yarn. Once the target shape had been obtained, the preform was hardened in its mold by reticulating the resin. After carbonization, the $\mathrm{C} / \mathrm{SiC}$ matrix was introduced using a Chemical Vapor Infiltration (CVI) process carried out in a specific furnace. Fig. 6.4 represents examples of flat and curved panels in hardened state. 


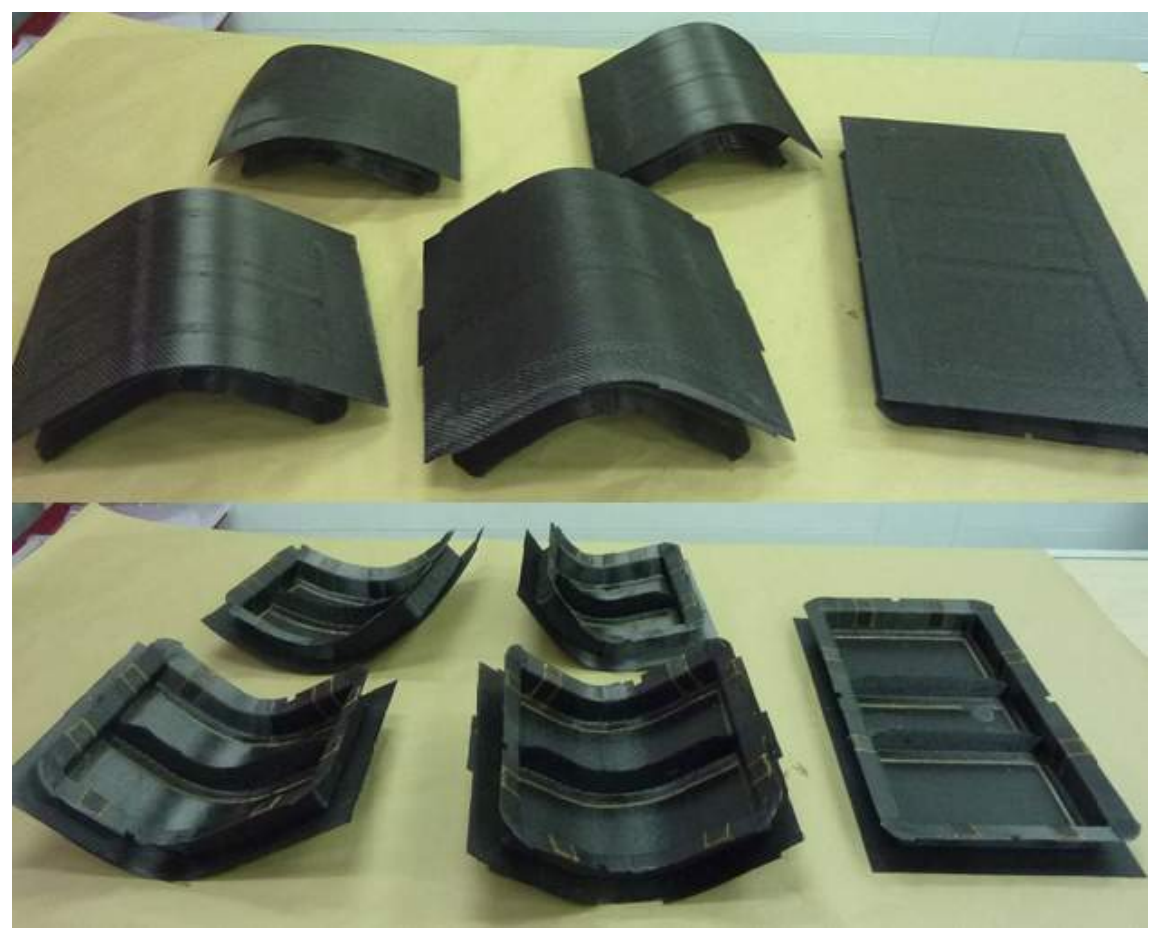

Figure 6.4.: CMC TPS panels in hardened state: external surface (top) and internal surface (bottom).

For the integration of CATE, shingles 3 and 5 were sent to LERMPS for Mullite coating application, prior to assembly of the inner layers of the TPS.

\subsubsection{Coating deposition by Vacuum Plasma Spraying}

The coating method chosen for CATE implementation is the Vacuum Plasma Spraying (VPS) technique. This method is normally applied in inert atmospheres at low pressure (from 10 to 200 mbar) to prevent substrate oxidation during the process. Mullite deposits produced by VPS are normally composed of a mixture of crystalline and amorphous phases due to the high cooling rate being imposed when molten particles reach the substrate. Since further crystallization of the amorphous phase can occur in service, leading to crack formation due to the volume variation, the strategy here was to obtain a fully crystallized Mullite by applying the coating in a furnace exceeding $1000^{\circ} \mathrm{C}$ [122]. Unfortunately, the shingle size coated in the present activity made this solution unfeasible for the CATE experiment. To overcome this limitation, an alternate method was followed. This was documented by Cojocaru et al. [123], who developed a method to produce as-sprayed crystallized Mullite coatings by using high enthalpy plasma torch and low stand-off distance. This method is based on the capacity of the plasma to locally transfer a high calorific power able to increase momentarily the substrate temperature over $1000^{\circ} \mathrm{C}$.

The Mullite coating adherence on CMC is often promoted by a silicon sub-layer deposition. This bond material is generally selected because of its chemical compatibility with $\mathrm{SiC}$ and its thermal expansion coefficient close to Si-based substrates. This configuration had been validated for turbine engine components under thermal cycling up to $1400^{\circ} \mathrm{C}$ [124, 125].

No specific surface preparation was made on the IXV shingles, except for dust removal by com- 
pressed air. To perform the coating in the selected area, a graphite mask previously machined was clamped precisely to the shingle. The choice of the mask material (graphite) was particularly important to ensure accuracy of the coated area with respect to the thermocouples positioning. To achieve sub-millimeter accuracy, it was necessary to avoid any deformation and to reduce thermal gradients with a good heat transfer to the CMC. A small C/SiC sample was placed over the mask to assess the quality of the coating after deposition, as shown in Fig. 6.5.

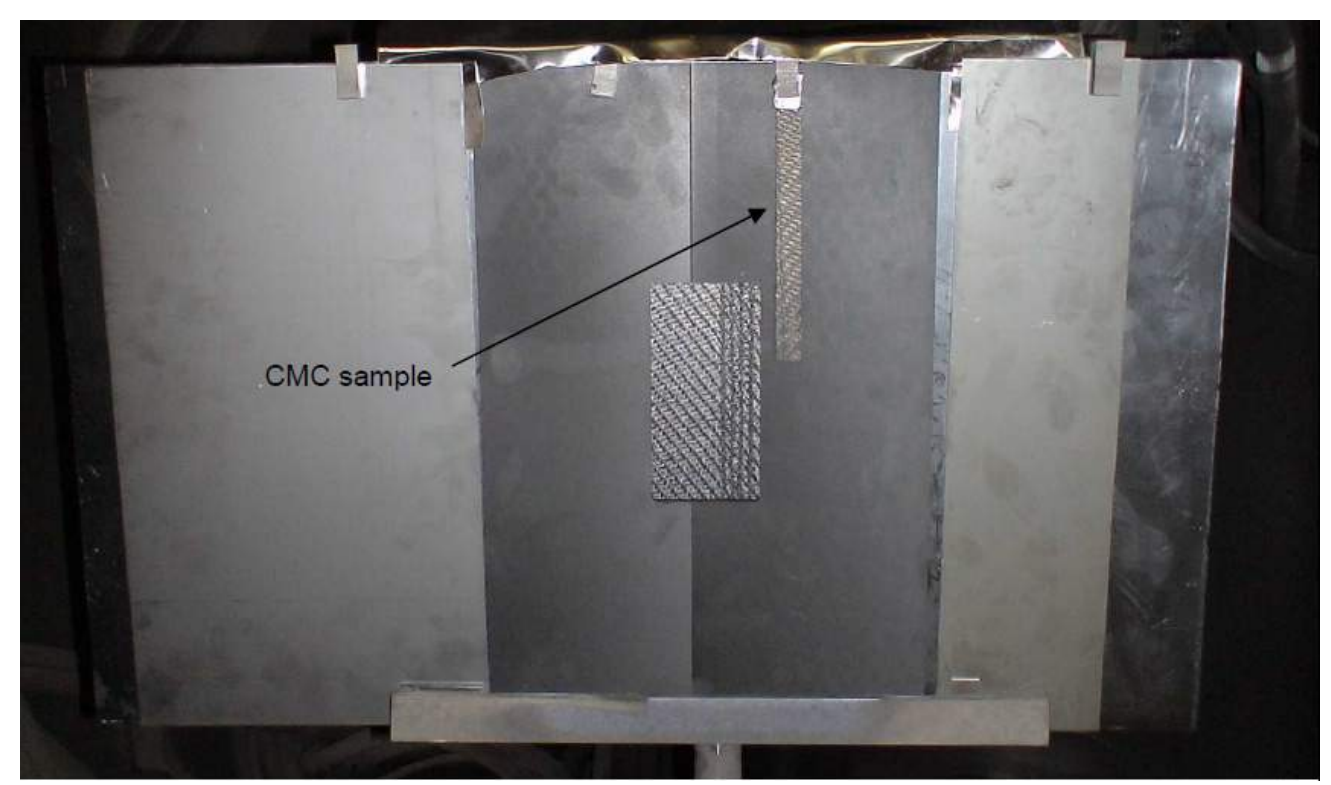

Figure 6.5.: Graphite mask on shingle 5 before coating with a CMC sample attached for qualification.

Coatings were performed using a $45 \mathrm{~kW}$ F4-VB plasma torch installed on a 6-axis robot in a VPS chamber. The feedstock materials were silicon and Mullite powders (\#1020) from Saint Gobain Coatings Solutions (Avignon, France). After the VPS atmosphere was pumped down and purged with argon, both substrate and mask were exposed to the plasma torch to increase their surface temperature. Then, plasma torch operating parameters were progressively turned to coating parameters, and the sub-layer material was fed in the torch followed by the Mullite. When the deposition sequence had finished, the shingle was kept inside the chamber until its temperature was below $50{ }^{\circ} \mathrm{C}$. The plasma spray parameters selected to produce the coatings are reported in Table 6.3.

The coated area is illustrated in Fig. 6.6, which shows well defined borders between coated and uncoated zones. A cross section Scanning Electron Microscope (SEM) image of the coating qualification sample is shown in Fig. 6.7. An average coating thickness of $\approx 68 \mu \mathrm{m}$ was obtained. A graded zone where the composition gradually evolves from Silicon to Mullite can be observed. Both coatings were relatively dense and uniform. Some pores appeared in the Mullite coating, but they were not supposed to affect the performance of CATE. 
Table 6.3.: Coating operational parameters

\begin{tabular}{lr}
\hline \hline Parameter & Value \\
\hline Arc intensity, A & 700 \\
Arc voltage, $\mathrm{V}$ & 68 \\
Primary gas $(\mathrm{Ar}), \mathrm{L} / \mathrm{min}$ & 50 \\
Secondary gas $\left(\mathrm{H}_{2}\right), \mathrm{L} / \mathrm{min}$ & 8 \\
Chamber pressure, $\mathrm{mbar}$ & 120 \\
Deposit temperature,${ }^{\circ} \mathrm{C}$ & 700 \\
\hline \hline
\end{tabular}

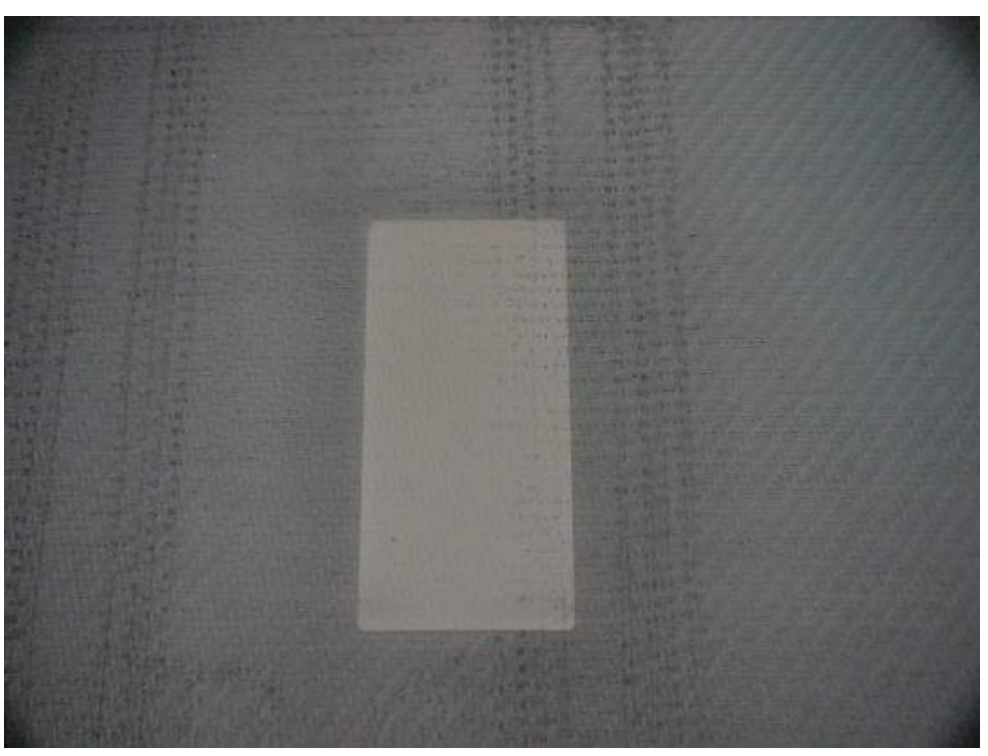

Figure 6.6.: Shingle with the Mullite patch.

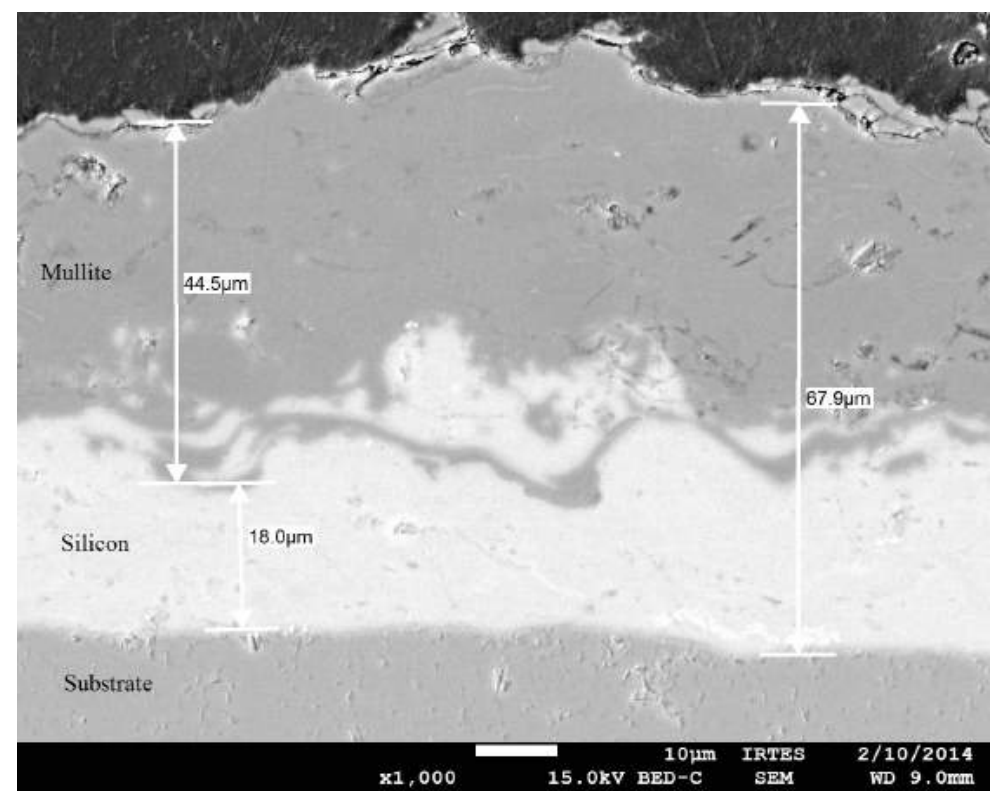

Figure 6.7.: Cross-section of the VPS coating. 


\subsubsection{Thermocouple integration and calibration}

All the shingle panels in IXV were instrumented with type-S thermocouples in selected locations. The number of thermocouples under the skin was increased on panels 3 and 5 , in the location of the catalytic patch, in order to track the temperature jump expected during re-entry. Thermocouples were integrated by RUAG Space on the CMC panels, using CMC bridges that had been previously installed by Safran-Herakles during the manufacturing process. The integration and routing of the thermocouples is shown in Fig. 6.8. This integration was performed before assembling the insulation layers, the seal and the attachments to the internal structure of the TPS.

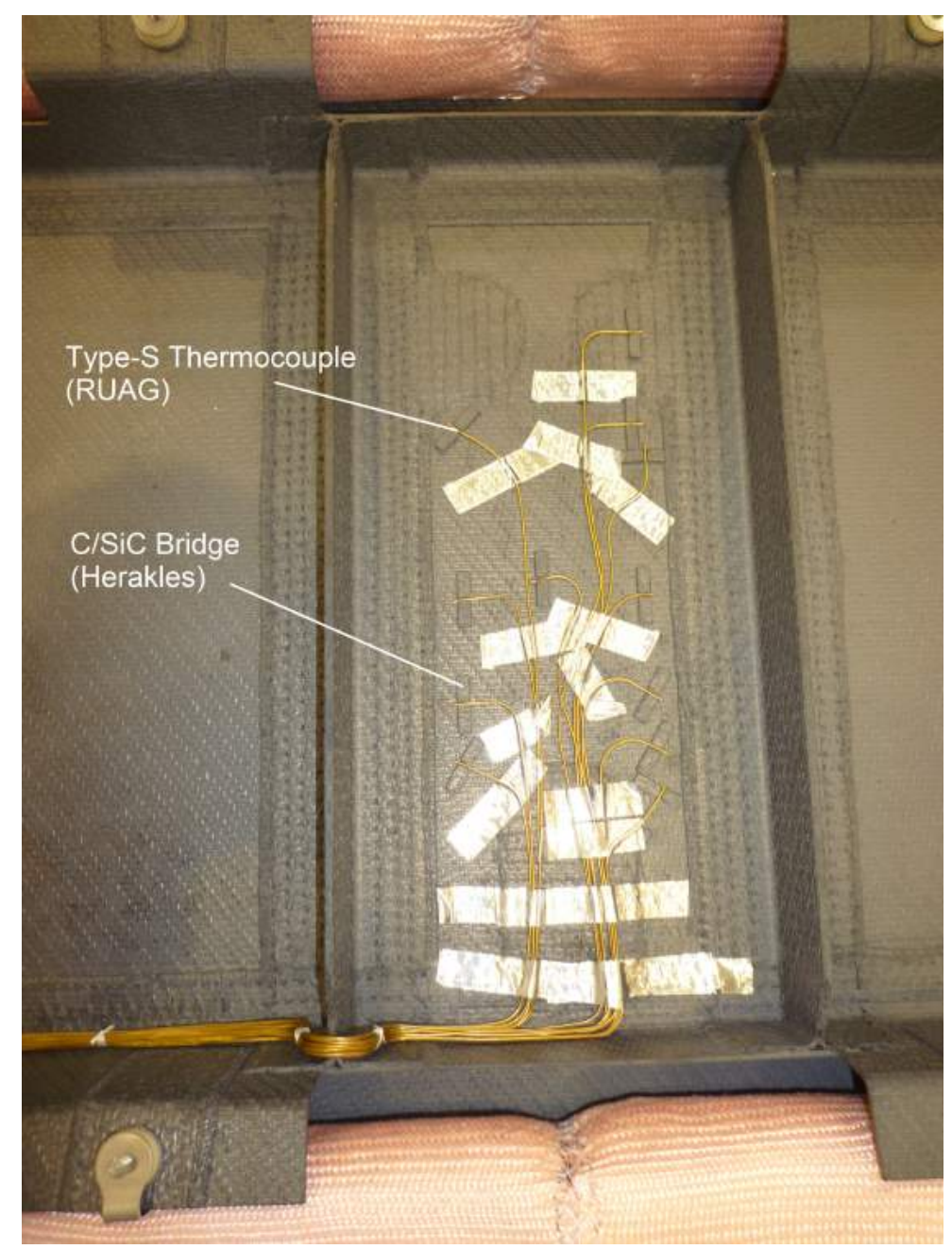

Figure 6.8.: Illustration of the $\mathrm{C} / \mathrm{SiC}$ bridges and thermocouple routing inside the TPS. The tape was replaced by ceramic-based glue prior to TPS assembly (photograph courtesy of TASI).

The assembled panel, instrumented with the thermocouples, was finally integrated in the vehicle. This delicate operation comprised the routing of the thermocouple wiring through the insulation, the cold structure and into the interior of the vehicle to its connection rack. Several operators had to simultaneously handle the panel to ensure that no damage occurred at any stage, while verifying the correct routing and connection of the parts. A final continuity check after integration allowed verifying the correct response of the thermocouples. TASI reported to VKI the damage on WT51 before launch. The final result of the TPS assembly in the windward side of IXV is shown in Fig. 6.9. 


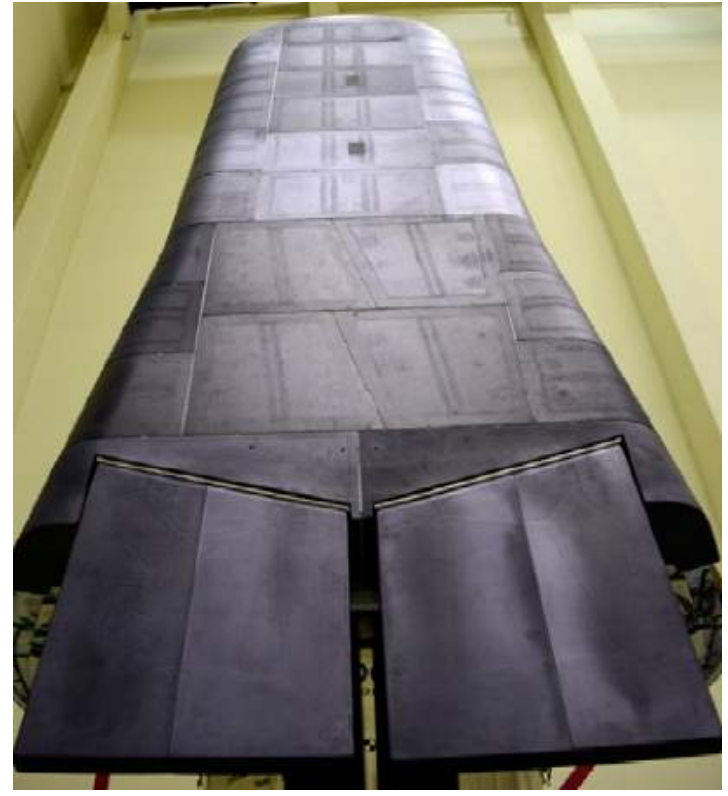

(a) Windward side.

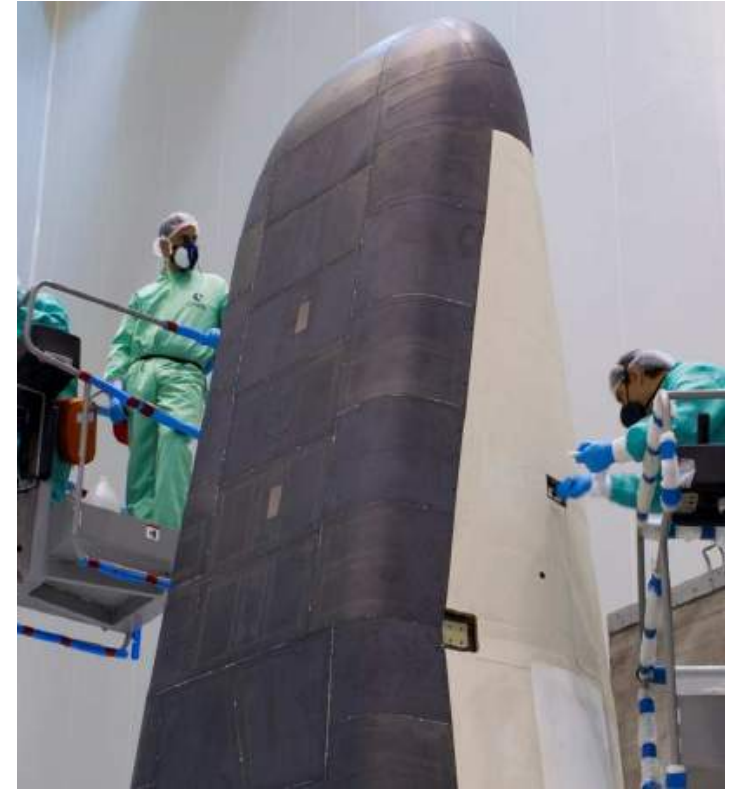

(b) Mullite patches.

Figure 6.9.: Final stage of IXV's assembly line (photograph courtesy of ESA).

\subsection{Preliminary IXV post-flight analysis}

After recovery in the Pacific Ocean, the IXV was brought back to Europe for post-flight inspection and analysis. Once on land, it began a tour around different European institutions that were involved in the mission. The VKI being one of them, IXV was presented to Belgian authorities and other industrial partners. A photograph of IXV after re-entry can be seen in Fig. 6.10.

A first glance to the vehicle shows the degradation of ablative TPS materials on the side walls. Future inspection of these materials will assist the development and validation of improved ablation models. It is interesting to see the caramel color at the top of the vehicle as consequence of flow detachment and re-circulation on the lee side. In contrast, the integrity of the $\mathrm{C} / \mathrm{SiC}$ nose cap demonstrates the suitability of this material as re-usable TPS. Indeed, no sign of degradation could be found on the surface with the naked eye. Unfortunately, visual access to CATE was not possible in the VKI, not only because of the vehicle's position on the platform, but also due to salt deposits (i.e. the white layer falling towards the bottom) that ended up covering the two patches.

The following lines provide a preliminary analysis of flight data delivered to industry partners. In particular, the processing is focused on the flight environment reproduction in the Plasmatron facility at both stagnation and off-stagnation point configurations. This is important to set the basis for a deeper post-flight analysis of IXV and CATE measurements concerning GSI phenomena. As a first step, trajectory data transferred to ground by telemetry is provided, together with thermocouple measurements along CATE, from which the most relevant flight conditions are identified.

\subsubsection{Stagnation point}

The re-entry path followed by IXV is shown in Fig. 6.11. Considering time from lift-off as reference, it is assumed to start at $t=3899 \mathrm{~s}$ and an altitude of $120 \mathrm{~km}$, where the velocity is reported as 7.44 


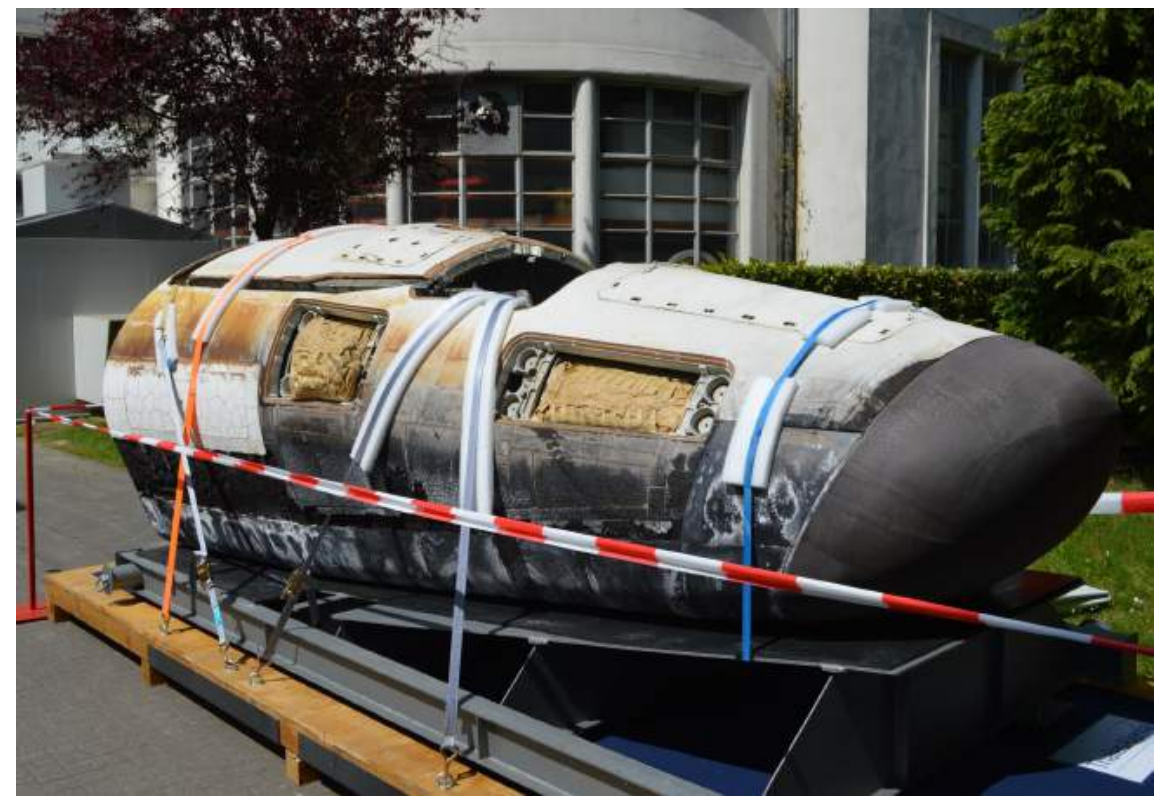

Figure 6.10.: Photograph of IXV after re-entry, taken at the VKI on June 2015.

$\mathrm{km} / \mathrm{s}$.

The peak heating is considered the most relevant parameter for analysis at stagnation point. Such condition is found in Fig. 6.11 using the correlation proposed by Brandis and Johnston [126] as:

$$
\dot{q}_{w}=7.455 \times 10^{-9} \rho_{\infty}^{0.4705} U_{\infty}^{3.089} R^{-0.52}
$$

This expression is limited to $3 \leqslant U_{\infty} \leqslant 9.5 \mathrm{~km} / \mathrm{s}$ and it is applied to IXV considering a nose radius $R$ of $1.2 \mathrm{~m}$. The free-stream density $\rho_{\infty}$, along with other thermodynamic state variables such as pressure and temperature, is estimated with the atmospheric model NRLMSISE-00. Data is corrected using the horizontal wind model HWM2007, as reported by TASI. Thus, the stagnation point peak heating is found at $70.18 \mathrm{~km}$ altitude and $t=4596 \mathrm{~s}$, which corresponds to the free-stream conditions specified in Table 6.4 .

Table 6.4.: IXV free-stream conditions at peak heating altitude

\begin{tabular}{lrrr}
\hline \hline$p_{\infty}$ & $T_{\infty}$ & $\rho_{\infty}$ & $U_{\infty}$ \\
$\mathrm{Pa}$ & $\mathrm{K}$ & $\mathrm{kgm}^{-3}$ & $\mathrm{~m} / \mathrm{s}$ \\
\hline 5.35 & 219.39 & $8.48 \times 10^{-5}$ & 6504.81 \\
\hline \hline
\end{tabular}

In order to reproduce the peak heating conditions on ground, the LHTS methodology presented in Sec. 2.3 is used. It is important to remember that the full stagnation point boundary layer duplication is achieved if total enthalpy, total pressure and external flow velocity gradient are respected between the two configurations. Both enthalpy and pressure are derived from Eqs. 2.17 and 2.18, respectively, and plotted in Fig. 6.12 for the whole IXV trajectory. The velocity gradient, on the other hand, is computed with Eq. 2.20, which requires $\rho_{e}=f\left(p_{e}, h_{e}\right)$ computed with Mutation ++ [71] under the assumption of LTE. 


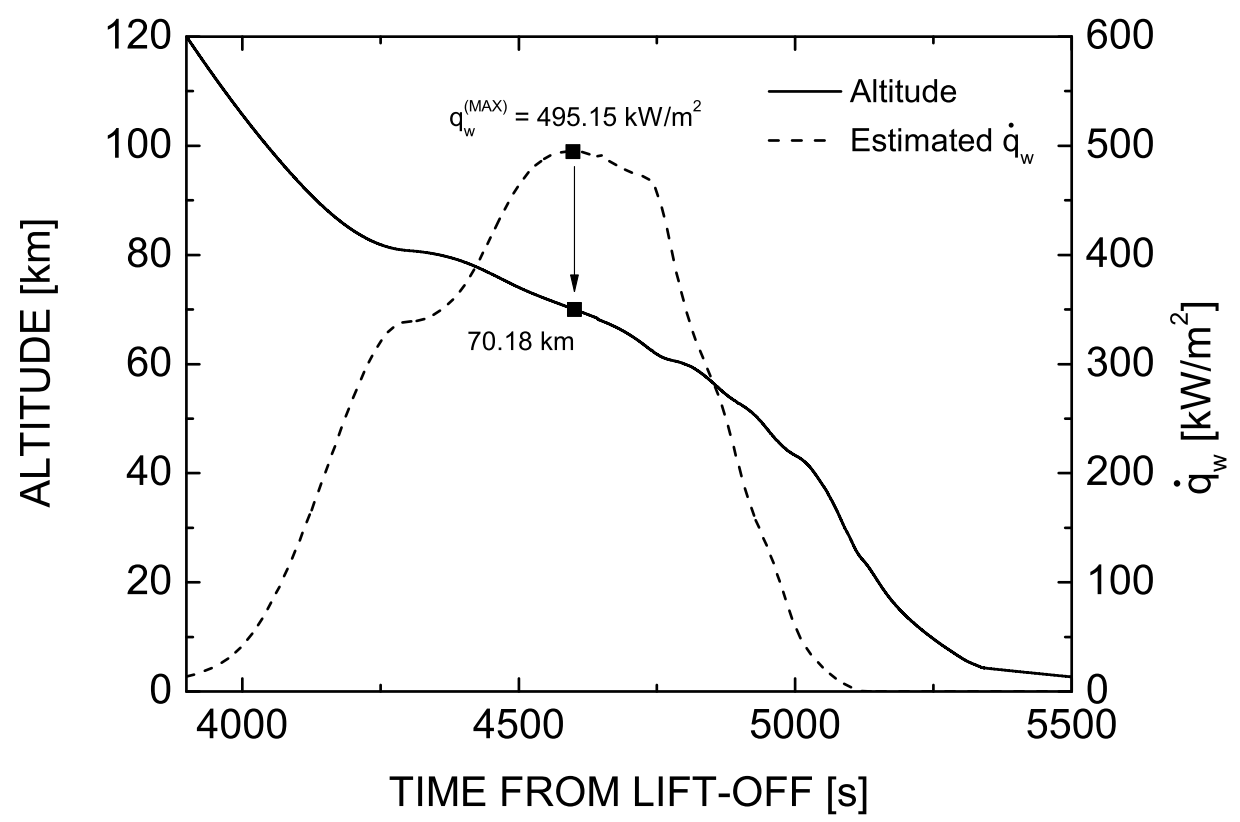

Figure 6.11.: Time evolution of IXV flight path and estimated stagnation point heat flux.

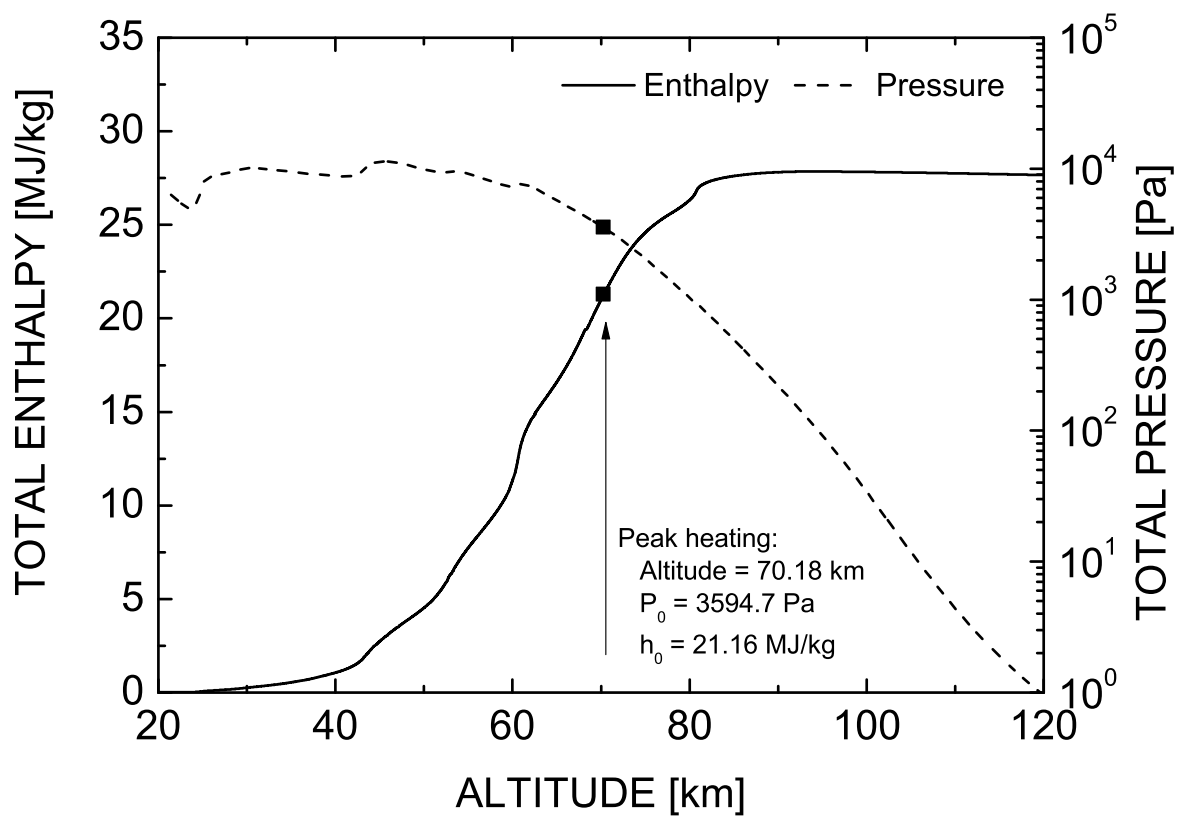

Figure 6.12.: Total enthalpy and total pressure as function of flight altitude. 


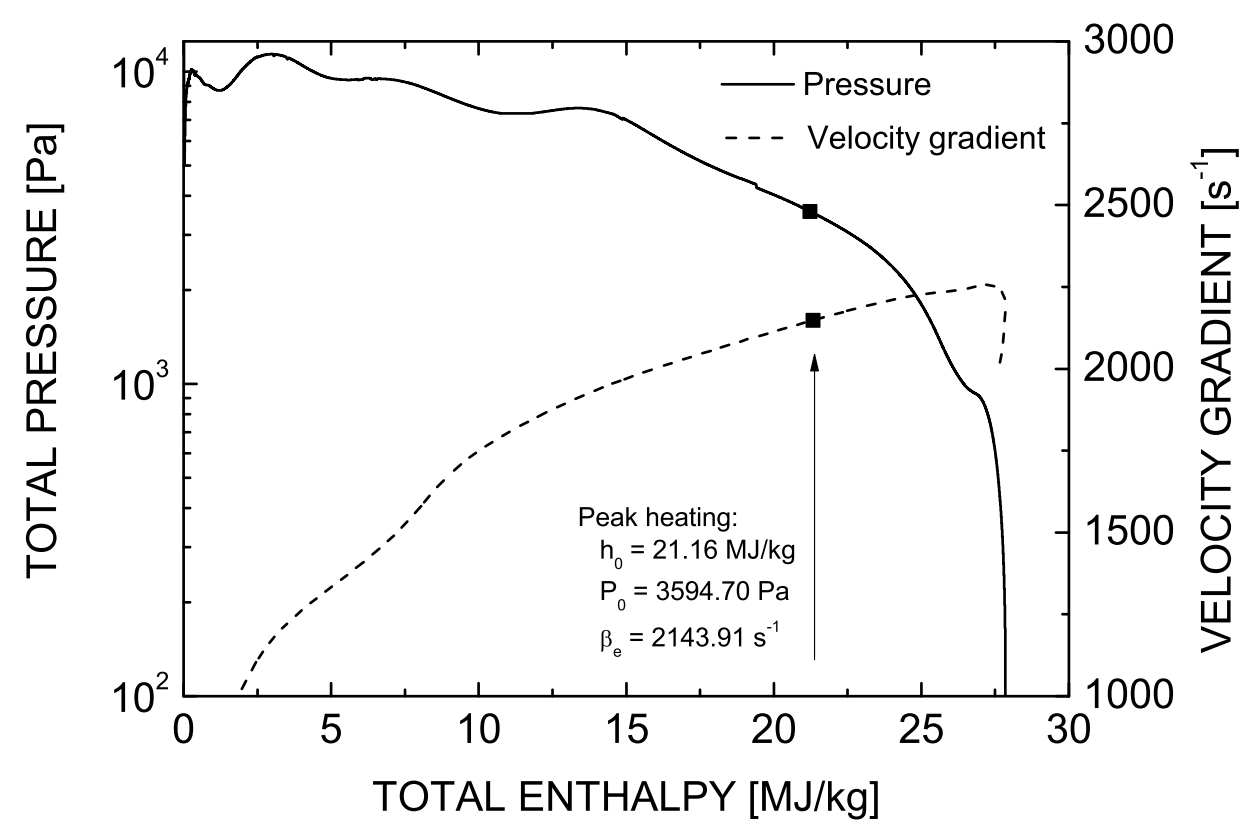

Figure 6.13.: Evolution of LHTS parameters during IXV re-entry.

The relation between the three extrapolation parameters is presented in Fig. 6.13. The extrapolation parameters are provided in Table 6.5 for IXV's peak heating condition.

Table 6.5.: LHTS parameters for IXV peak heating conditions

\begin{tabular}{lrr}
\hline \hline$h_{e}^{f}$ & $p_{e}^{f}$ & $\beta_{e}^{f}$ \\
$\mathrm{MJ} / \mathrm{kg}$ & $\mathrm{Pa}$ & $\mathrm{s}^{-1}$ \\
\hline 21.16 & 3594.70 & 2143.91 \\
\hline \hline
\end{tabular}

Note that, while pressure and enthalpy can be easily defined for test condition definition on ground, an additional exercise should be carried out to find an appropriate test model radius that represents the velocity gradient of IXV in a test chamber. This activity goes beyond the scope of this thesis and will not be developed here, but it should be done in two steps: the definition of an equivalent sphere under hypersonic regime followed by another one for subsonic flow using Eq. 2.23 .

\subsubsection{Off-stagnation point: CATE}

The catalytic jump implemented on the windward side of IXV can bring valuable information about the catalytic activity on the TPS surface downstream from the stagnation point. After an adequate post-processing, thermocouple data could be used to extract the recombination coefficient during re-entry on the surroundings of CATE, which would allow catalytic model validation in plasma facilities. A proper quantification of the recombination coefficient $\gamma$ during IXV re-entry is critical 
for that purpose, making the off-stagnation point extrapolation methodology presented in $\mathrm{Ch}$. 5 extremely useful.

Relevant flight data provided by CATE is presented below. Although the recombination coefficient cannot be determined without a thorough post-processing, the collected experimental data shown hereafter already demonstrates the success of the experiment regarding the catalytic jump evidence.

The focus is driven towards the catalytic transitions caused by the two Mullite patches when treating the flight data. The low-to-high catalytic transition occurs between thermocouples WT23 and WT24 on shingle 3, and between WT50 and WT52 on shingle 5. The high-to-low transition, on the other hand, falls between WT27 and WT28 on shingle 3 and between WT54 and WT55 on shingle 5. The time evolution of the thermocouple measurements is shown in Fig. 6.14 and Fig. 6.15 for shingles 3 and 5, respectively. Note that the location of the thermocouples is specified in Table 6.1. It is important to remind the reader that the catalytic transition on shingle 5 actually occurs upstream of WT51, but TASI reported WT51 to be damaged during assembly and, in consequence, this thermocouple must not be considered for post-processing.

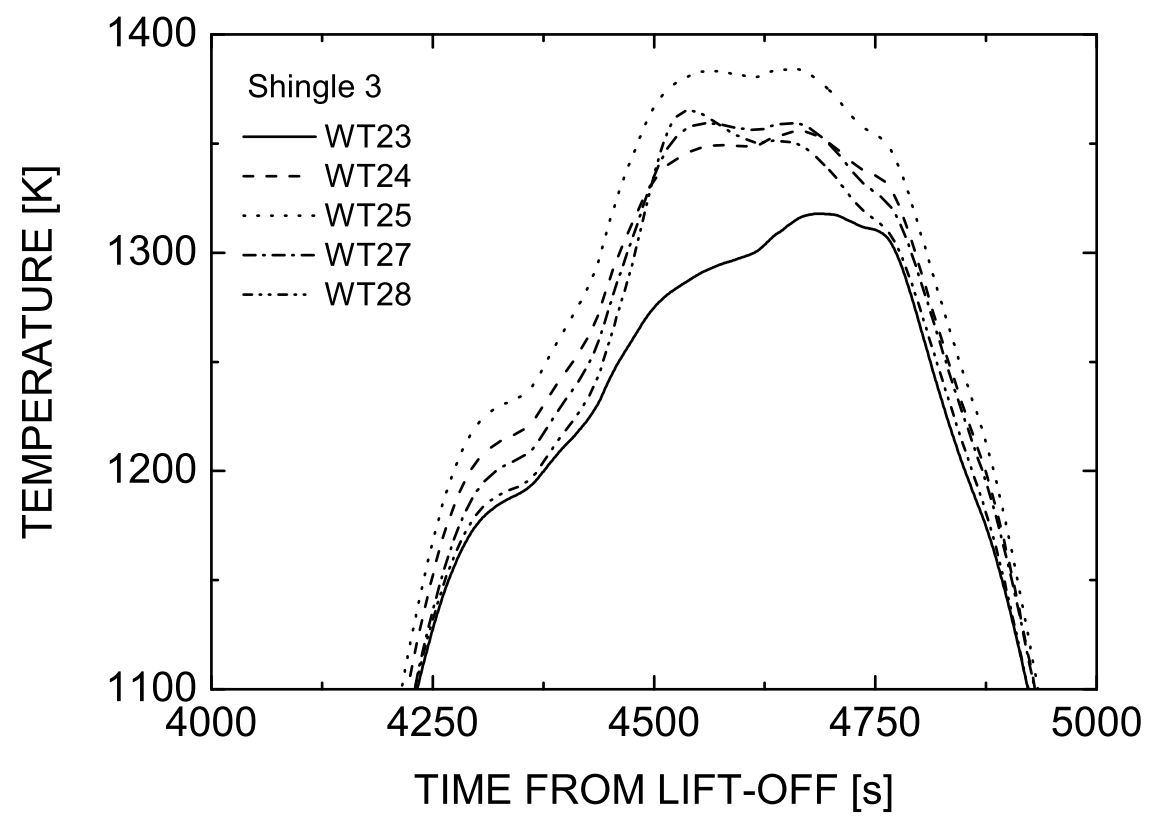

Figure 6.14.: Time evolution of measured temperatures by thermocouples close to both low-to-high and high-to-low catalytic transitions on shingle 3. 


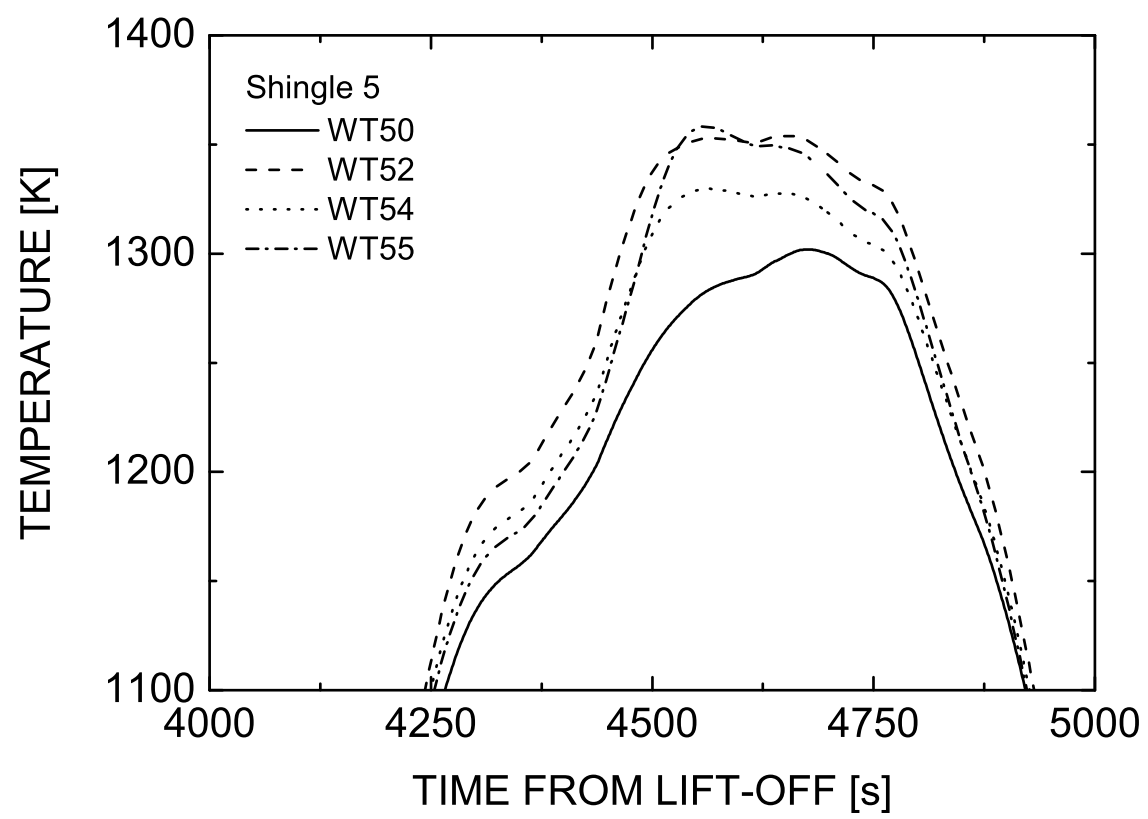

Figure 6.15.: Time evolution of measured temperatures by thermocouples close to both low-to-high and high-to-low catalytic transitions on shingle 5.

The trajectory points considered of major interest for post-flight activities are those with the highest surface temperature difference between two consecutive thermocouples. This is expected to help with the detection of the flight conditions that are most sensitive to catalysis. The temperature jumps $\Delta T$ induced by the two catalytic transitions are plotted in Fig. 6.16 for the first patch, and in Fig. 6.17 for the second one. As expected, the low-to-high catalytic transition leads to a higher $\Delta T$ than the high-to-low transition. Remark also that there are situations where $\Delta T<0$ for the highto-low catalytic transition, which means that temperature on $\mathrm{C} / \mathrm{SiC}$ is higher than on Mullite. This temperature jump, reaching $20 \mathrm{~K}$, is well outside the thermocouples' uncertainty margins, which are reported to be of $\pm 0.25 \%$. One possible explanation for such result will be given afterwards.

Taking the maximum $\Delta T$ for the low-to-high catalytic transition as reference, the corresponding flight conditions are reported in Table 6.6 for shingles 3 and 5. The maximum temperature jump is $59.25 \mathrm{~K}$ for the former and $81.51 \mathrm{~K}$ for the latter. Remark that $\Delta T^{(M A X)}$ on shingle 5 is higher than on shingle 3 , probably because it occurs at a higher free-stream velocity, which leads to a frozen boundary layer and increases heat flux sensitivity to catalytic reactions.

Table 6.6.: IXV free-stream conditions for $\Delta T^{(M A X)}$ over the low-to-high catalytic transition

\begin{tabular}{lrrrrrr}
\hline \hline & $\mathrm{t}$ & $\begin{array}{r}\text { Altitude } \\
\mathrm{km}\end{array}$ & $\begin{array}{r}p_{\infty} \\
\mathrm{Pa}\end{array}$ & $\begin{array}{r}T_{\infty} \\
\mathrm{K}\end{array}$ & $\begin{array}{r}\rho_{\infty} \\
\mathrm{kgm}^{-3}\end{array}$ & $\begin{array}{r}U_{\infty} \\
\mathrm{m} / \mathrm{s}\end{array}$ \\
\hline Shingle 3 & 4521 & 73.11 & 3.40 & 214.39 & $5.52 \times 10^{-5}$ & 6863.10 \\
Shingle 5 & 4500 & 74.05 & 2.94 & 212.78 & $4.80 \times 10^{-5}$ & 6945.45 \\
\hline \hline
\end{tabular}




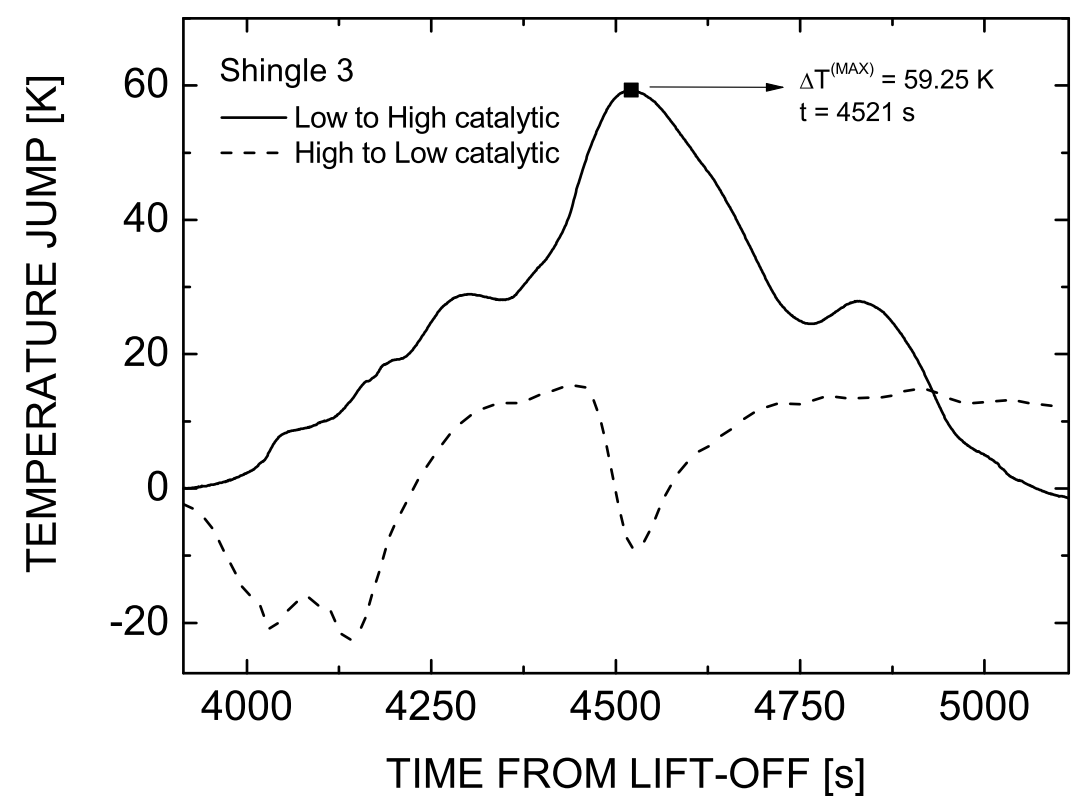

Figure 6.16.: Time evolution of temperature jump across the two catalytic transitions in shingle 3 .

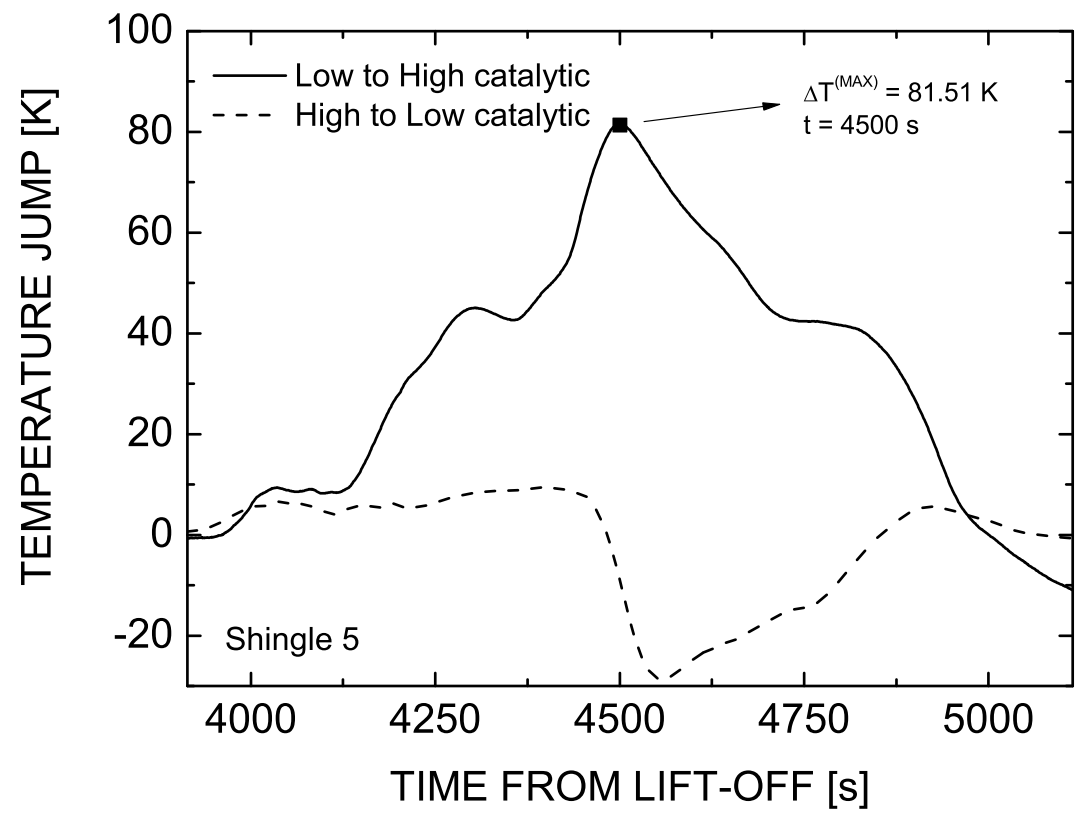

Figure 6.17.: Time evolution of temperature jump across the two catalytic transitions in shingle 5 .

The suite of temperature measurements provided by CATE at the flight conditions reported in Table 6.6 are plotted in Fig. 6.18 and Fig. 6.19, for shingles 3 and 5, respectively. Remark that these are the raw temperature measurements coming from the S-thermocouples placed behind the 
CMC panels, and not the tempertaures of the surface in contact with the gas. However, this is not supposed to be a problem to extract relevant information from the experiment at this intial stage of the post-flight activity. For instance, the temperature jump caused by the presence of a high catalytic patch leads to the conclusion that the baseline $\mathrm{C} / \mathrm{SiC}$ is not full-catalytic. This is very important given the fact that such assumption is normally made during the design of a TPS, so it encourages working towards a more efficient design for future missions.

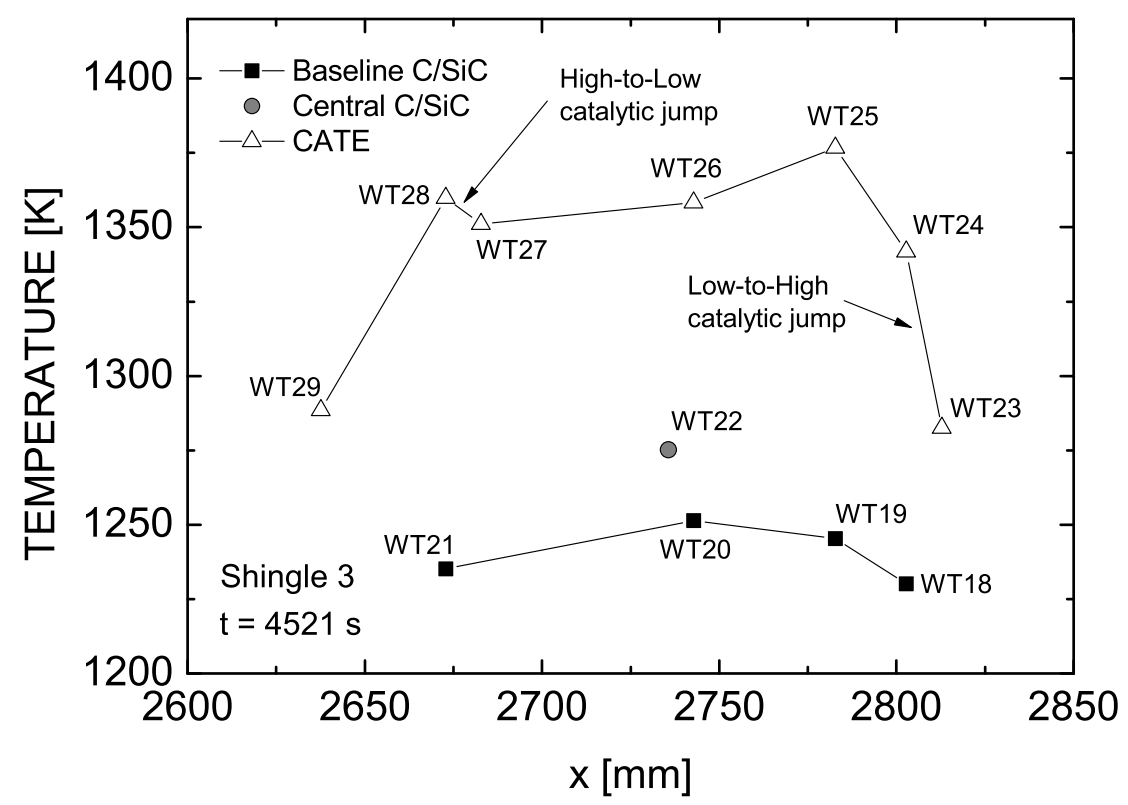

Figure 6.18.: CATE temperature measurements at $t=4521 \mathrm{~s}$ for shingle 3 .

Despite the abruptness of the catalytic transition, it is observed that the temperature jump is spread over a wider area. This behavior was not anticipated by CFD computations [127. One possible explanation for the smooth temperature increase across the catalytic discontinuity could be thermal conduction inside the CMC panel, which was not taken into account during computations. Thus, an energy transfer from the patch towards the baseline ceramic would appear, leading to temperature distributions that differ from CFD. The fact that temperature also increases along the $x$ coordinate on the other side of the symmetry plane (following the temperature distribution on the patch), together with the temperature gradient observed in the direction perpendicular to this symmetry plane by WT22 and WT48, strengthens such hypothesis.

Another issue is the peak temperature detected by WT28 and WT55 on shingles 3 and 5, respectively. These two thermocouples, located over the baseline material, measure a temperature that is higher than the reading of the thermocouple just upstream the high-to-low catalytic transition. This was not anticipated by CFD either. Although a peak in temperature close to the transition could be caused by atoms diffusing upstream of the catalytic jump, the peak should still remain in the highly catalytic patch. One the other hand, one should note also that the maximum temperature is always registered by the second thermocouple on the Mullite patch for shingle 3 (i.e. WT25) downstream of the low-to-high catalytic transition. Then, it would seem that there is a persistent 


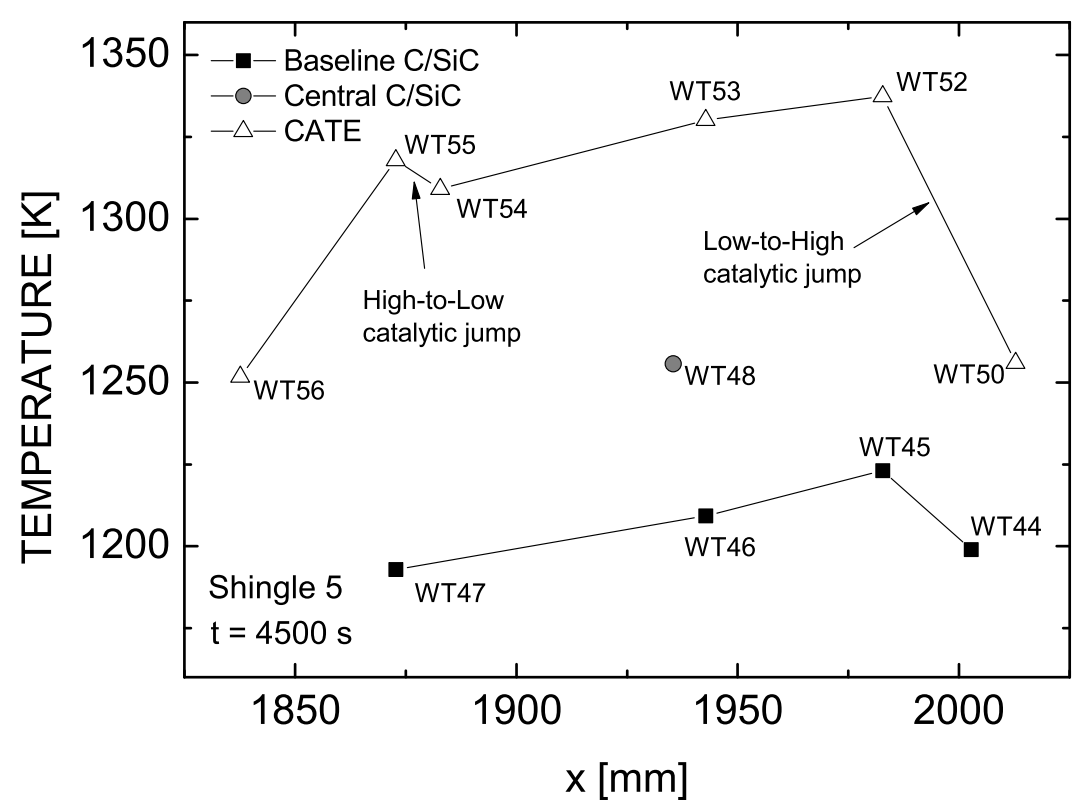

Figure 6.19.: CATE temperature measurements at $t=4500 \mathrm{~s}$ for shingle 5 .

delay between the peak temperature and the transition. This could be explained by a finite Damköhler number of the flow at off-stagnation point configuration. Indeed, if the characteristic time of the catalytic reaction is in the same order as the characteristic convective time for the flow, a delay would appear between the catalytic jump and the diffusive heat flux due to catalysis, shifting the peak temperature downstream. Therefore, one could conclude that peaks in WT28 and WT55 could be consequence of a coupled effect between convection and diffusion, both in the direction parallel to the flow.

\subsubsection{Thermal analysis for CATE post-processing}

Data provided by CATE correspond to thermocouple measurements on IXV. However, it is important to remember that these thermocouples are installed on the inner side of the $\mathrm{C} / \mathrm{SiC}$ shingles, and that their readings could significantly differ from the actual temperature values on the external surface in contact with the gas, due to thermal conduction through the TPS. Therefore, and before proceeding with any aerothermodynamic model validation campaign, one must ensure that thermocouple measurements of CATE can be confidently related to surface temperatures on the other side of the shingles.

This section attempts to validate the hypothesis stating that the temperture measured by thermocouples in CATE $\left(T_{T C}\right)$ equals the surface temperature in contact with the gas $\left(T_{w}\right)$. Note that such hypothesis falling true is rather convenient because it would make the heat transfer by conduction inside the $\mathrm{C} / \mathrm{SiC}$ shingles much smaller than the heat emited by radiation, and the energy balance on the surface as proposed in Eq. 1.1 would remain correct for numerical solvers. On the contrary, if the hypothesis turns false, the CFD simulations for IXV would need to be coupled with thermo-mechanical models of the solid phase to reproduce the thermal response of the TPS 
in relevant flight conditions, which would increase significantly the time and cost of the flight data post-processing.

Two aspects are considered here to validate the hypothesis $T_{w}=T_{T C}$. The former is the contribution of heat transfer by conduction $\left(\dot{q}_{\text {cond }}\right)$ inside the $\mathrm{C} / \mathrm{SiC}$ panel in steady state conditions to the total heat flux exchanged on the surface in contact with the gas $\left(\dot{q}_{w}\right)$. The latter is the temperature difference $T_{w}-T_{T C}$ that could be expected due to the unsteady nature of the re-entry, which varies the free-stream conditions at different flight altitudes, and could introduce a shift (or delay) between the actual $T_{w}$ and the $T_{T C}$ readings at a specific time $t$ if thermal conductivity of $\mathrm{C} / \mathrm{SiC}$ is not high enough.

First, in order to neglect the heat flux conducted inside the $\mathrm{C} / \mathrm{SiC}$ shingle, one should verify that $\dot{q}_{\text {cond }}$ is negligible against the heat transferred on the surface $\dot{q}_{w}$. Assuming the $\mathrm{C} / \mathrm{SiC}$ shingle as a flat plate, which is reasonable considering that its thickness $(3 \mathrm{~mm})$ is much smaller than the other two dimensions $(677 \times 398 \mathrm{~mm})$, the condition $B i<0.1$ should be satisfied to neglect $\dot{q}_{\text {cond }}$, whith $B i$ being the Biot number:

$$
B i=\frac{h \delta}{\lambda}
$$

where $h, \delta$ and $\lambda$ are the convective heat transfer coefficient, the thickness of the panel and the thermal conductivity of the solid phase, respectively. The convective heat transfer coefficient is defined as $h=\dot{q}_{w} /\left(T_{e}-T_{w}\right)$ and it can be estimated through a numerical simulation of the IXV. Such simulation is carried out with $\mathrm{CFD}++$ software, using the same mesh and configuration as that used in Sec. 5.2, but with the inlet conditions specified in Table 6.6 for shingle 3. The wall is assumed fully catalytic and in radiative equilibrium with an emissivity of 0.8. Simulation results provide $T_{e}=6230 \mathrm{~K}, T_{w}=1475 \mathrm{~K}$ and $\dot{q}_{w}=214.55 \mathrm{~kW} / \mathrm{m}^{2}$ close to shingle 3 , yielding to $h=45.12$ $\mathrm{W} / \mathrm{m}^{2} \mathrm{~K}$. The thermal conductivity of $\mathrm{C} / \mathrm{SiC}$ is reported to be $\lambda_{C / S i C} \approx 125 \mathrm{~W} / \mathrm{mK}$ in Ref. [128, which leads to $B i=1.08 \times 10^{-3}<<0.1$, and validates the hypothesis of $T_{w}=T_{T C}$ in steady state. Note that, although similar values of $\lambda_{C / S i C}$ are reported by literature, the thermal conductivity of $\mathrm{C} / \mathrm{SiC}$ is expeted to be highly anisotropic, so its direction with respect to the carbon fibres remains unknown. Nonetheless, and since $B i$ is two orders of magnitude lower than 0.1 , the error in $\lambda_{C / S i C}$ is not expected to lead towards a different conclusion.

Next, one should take into account that the heat conduction through $\mathrm{C} / \mathrm{SiC}$ might be slow compared to the variation of the free-stream conditions during the re-entry, which would introduce a shift (or delay) between the temperature readings $\left(T_{T C}\right)$ and the surface temperatures $\left(T_{w}\right)$ at each specific time $t$. This aspect can be addressed considering the unsteady heat conduction equation and, more specifically, evaluating the Fourier number on $\mathrm{C} / \mathrm{SiC}$. The Fourier number is defined as:

$$
F_{O}=\frac{\lambda \Delta t}{\rho c_{p} \delta^{2}}
$$

where $\rho$ and $c_{p}$ are the material density and specific heat, respectively, and $\Delta t$ is a characteristic time of the unsteady boundary condition. Therefore, the Fourier number represents how fast the thermal gradients propagate through the material with respect to $\Delta t$. Thus, if $F o>1$ the heat propagates much faster than the variation of the wall condition and it would be reasonable to assume 
the thermal problem in steady state, hence $T_{w}=T_{T C}$. On the contrary, if $F_{o}<<1$ the thermal wave does not propagate fast enough and a shift in temperatures could be expected between the two boundaries. In CATE, thermocouple measurements are taken at $1 \mathrm{~Hz}$, therefore $\Delta t=1 \mathrm{~s}$ is taken as reference. Then, $\rho=205 \mathrm{~kg} / \mathrm{m}^{3}$ and $c_{p}=750 \mathrm{~J} / \mathrm{kgK}$ for $\mathrm{C} / \mathrm{SiC}$, leading to $F o=90.33$. Since this number is much higher than unity, the problem at hand can be considered in steady state, and the assumption of $T_{w}=T_{T C}$ remains valid in $\mathrm{C} / \mathrm{SiC}$ for CATE post-processing.

Note that these results show that the layer of $\mathrm{C} / \mathrm{SiC}$ in the TPS does not contribute to the insulation of IXV's payload, but to the structural integrity in the high temperature environment encountered during re-entry. Instead, the insulation is actually achieved by the inner layers stacked inside the TPS.

\subsection{Future perspective of CATE}

The previous section gave a first glance to the raw data provided by IXV and CATE. However, a lot of work should be carried out in the near future to understand the phenomena observed across the catalytic transition and to start considering flight data for aerothermodynamics model validation.

Post-flight activities should include a thorough study and a deeper understanding on both species diffusion phenomena in the gas and thermal conduction inside the whole TPS. This could be addressed with simulations that couple CFD with material thermo-mechanical properties. Such simulation could be used also to assess the thermal response, so that steady-state tests in plasma wind tunnels can be representative of the actual unsteady situation found in re-entry. This problem was partly addressed by de Cáceres [129] in the Plasmatron.

Another issue that must be addressed is how to measure the recombination coefficient at offstagnation point on a TPS material sample. In this thesis, recombination coefficients at offstagnation point are provided for an isothermal wall at $350 \mathrm{~K}$. However, testing with a TPS might be different, given the fact that surface temperature is not only influenced by GSI phenomena, but also by the thermal properties of insulating layers inside a TPS. In addition, the fact that the flat plate model available in the Plasmatron is much thinner than the TPS, requires finding an equivalent insulator that provides the same surface temperatures on $\mathrm{C} / \mathrm{SiC}$ as if it were the real TPS configuration.

To conclude, and considering all the issues mentioned above, one could finally apply the offstagnation point extrapolation methodology developed in Ch. 5 in order to define the Plasmatron testing conditions that reproduce the same flight environment found on CATE and, from them, estimate the recombination coefficient in flight. 


\section{Chapter 7.}

\section{Conclusions}

This thesis assesses plasma wind tunnel capabilities for catalytic model development and validation. It provides a better understanding on the role of species diffusion during the quantification process of catalytic recombination coefficients of TPS materials, and develops an innovative methodology for flight to ground extrapolation of non-equilibrium boundary layers that appear downstream of the stagnation point of a flying body. The work is being developed in the framework of IXV, focusing on the onboard experiment CATE which provides relevant flight data that could be used for future aerothermodynamic model validation.

\subsection{Contribution}

A general overview of different approaches to model catalysis is presented in this thesis. This contributes at understanding the capabilities and limitations of current modelling methods. Specific literature tends to follow two different perspectives. One is the so-called macroscopic description ( $\gamma$-models) of catalysis, which models the recombination coefficient $\gamma$ as a parameter proportional to the reaction rate constant $k_{w}$, and the microscopic description, which models through FRC the catalytic process in several reaction mechanisms with independently defined reaction rates. The former approach assumes that surface reactions behave as first order, implying that reaction rate constants can be a function of temperature only. The latter, on the other hand, leads to a recombination coefficient which is a result of the mass balance at the surface and, in consequence, they are compatible with second order effects through the Langmuir-Hinshelwood mechanism.

A revision of some dedicated plasma facilities is also given. They are extremely useful for catalytic model validation. Generally, they can be classified as diffusion tubes and plasma wind tunnels, and their working principles are very different. For instance, while diffusion tubes focus on the analysis of species diffusion caused by surface reactions, flows provided by plasma wind tunnels are coupled with convective phenomena.

Considering catalytic determination, diffusion tubes use spectroscopic techniques for plasma gas diagnostics around a sample, retrieving atomic species gradients that lead to the recombination coefficient, and therefore restricting their measurements to the frozen flow hypothesis. Plasma wind tunnels, on the other hand, rely on heat flux measurements coupled with CFD solvers to determine $\gamma$. Since the latter operate closer to the flight peak heating conditions, where pressure is not as low as that reached by diffusion reactors (only a few Pascals), the recombination coefficients measured in plasma wind tunnels can be influenced by non-equilibrium phenomena inside the boundary layer, such as the gas-phase Damköhler number, which are not relevant in diffusion tubes. 
The present work contributes at demonstrating that boundary layer non-equilibrium effects are, indeed, influencing the catalytic characterization of TPS materials in plasma wind tunnels such as the VKI-Plasmatron. This is done through two testing campaigns on copper calorimeters at constant surface temperature $(350 \mathrm{~K})$. The first one assesses the influence of both pressure and boundary layer edge enthalpy conditions, and the second one evaluates the level of gas recombination at constant pressure under different probe geometries: at stagnation and off-stagnation point configurations. The former campaign is used to build a reference recombination coefficient model $\gamma_{r e f}$ that allows rebuilding Plasmatron free-stream conditions, and the latter isolates the probe configuration to observe its effect on the catalytic determination at the same pressure, edge enthalpy and surface temperature conditions. Note that a method for catalytic determination at off-stagnation point is developed here for the first time in VKI.

Test results encourage the development of a flight to ground extrapolation technique at offstagnation point. This is carried out in the framework of the catalytic experiment CATE on board of IXV, and presented in Ch. 5. A new methodology is proposed, where the local boundary layer at a specific point of IXV's wall can be obtained at the trailing edge of a flat plate model that fits inside the Plasmatron. This extrapolation activity is critical for post-flight analysis of data provided by CATE, aiming at aerothermodynamic model validation in the near future.

As final contribution, the design and implementation of CATE on the windward TPS of IXV is described, and relevant flight data provided by CATE are reported. The purpose is to make data easily available for the post-flight activities of IXV. Emphasis is put on the definition of the extrapolating variables at both stagnation and off-stagnation point configurations.

\subsection{Conclusions}

From the literature review, one could conclude that the main advantage of diffusion reactors for catalytic quantification lies on the fact that they work at very low pressures. This means that, in contrast with plasma wind tunnels, they can assume flows with much less atomic recombination in the gas. The frozen flow regimes are known as the most suitable conditions for the determination of recombination coefficients. Conversely, plasma wind tunnels operate under environments much closer to peak heating conditions, where pressure is not as low as in diffusion tubes, and frozen boundary layers are not always guaranteed. Because catalytic measurements in these two types of facilities differ significantly, a problem arises concerning the use of an experimentally determined recombination coefficient in a CFD re-entry simulation. Indeed, one could use the value obtained in diffusion reactors and neglect the effect of gas-phase reactions on catalysis, or one could simply use the recombination coefficient measured in a plasma wind tunnel under relevant temperature and pressure conditions closer to those expected in the simulation.

Many commercial CFD softwares include the option to specify recombination coefficients on catalytic walls to define the boundary condition for the species equations. The CFD ++ software used in this thesis is one example. However, constant values of catalycity imposed along the vehicle's wall are not compatible with the temperature and pressure distributions around the body. Therefore, and answering the question postulated in Sec. 1.4. one cannot prescribe the same recombination coefficient across the complete wall of a vehicle because an important effect of temperature and 
pressure is observed during the catalytic characterization of TPS materials in plasma wind tunnels. The influence of pressure on catalysis determination could be consequence of two phenomena: either the first order reaction hypothesis at the wall is not correct, or/and the gas-phase recombination (not frozen flow) can reduce the mass fractions of atomic species close to the wall, making the catalytic conditions of the surface irrelevant for heat transfer.

Despite the pressure effect is incompatible with the first order reaction assumption of $\gamma$-models, such hypothesis can be avoided by newer FRC models. However, the fact that the latter are not widely implemented in CFD solvers, and that their complexity makes them difficult to validate in plasma facilities, makes the former far from obsolete. Therefore, there is still room for $\gamma$-models in the framework of TPS design as long as they are combined with a proper flight extrapolation strategy of the boundary layer.

This thesis provides a better understanding on how the non-equilibrium effects can affect the recombination coefficient determination at constant temperature. This is done through two test campaigns: one to demonstrate the effect of pressure, and the other to assess the effect of the probe geometry and flow configuration on $\gamma$.

\subsubsection{The Mini-Max}

The recombination coefficient on TPS materials cannot be determined by the VKI non-equilibrium boundary layer solver (CERBOULA) without previously knowing the bounadry layer edge conditions. At the same time, the edge conditions cannot be computed without the recombination coefficient on a reference material. The methodology proposed in this thesis allows determining a reference recombination coefficient on cold-wall metallic calorimeters using the Plasmatron. Note that testing on metallic calorimeters is advantageous because, since they are water-cooled, they remain at the same temperature over a large range of the Plasmatron envelope.

The Mini-Max method allows building a reference catalytic model $\gamma_{r e f}$ from which the edge enthalpy $H_{e}$ of the plasma can be rebuilt using CERBOULA. The Mini-Max relies on three heat flux measurements on three different calorimeters: silver, copper and Quartz. The logic behind the method is that, heat flux differences being only induced by different catalycities, silver is assumed the closest to full-catalytic and Quartz the closest to non-catalytic in order to define the enthalpy range of a specific Plasmatron condition. Then, this enthapy range can be used to define a range on copper catalycity inside which $\gamma_{r e f}$ should fall.

Applying the method under different power and pressure conditions can isolate the effect of these two parameters on the recombination coefficient determination for copper at $350 \mathrm{~K}$. Note that this parametric study can not be realized on TPS material samples due to the fact that their surface temperature varies with the Plasmatron test conditions. Results show a minimum dependence of $\gamma_{r e f}$ on $H_{e}$, and a strong influence of pressure. Therefore, a reference recombination coefficient $\gamma_{r e f}\left(P_{s}\right)$ on copper at $350 \mathrm{~K}$ is proposed in Sec. 3.5. This $\gamma$-model is further used for free-stream characterization during the next campaigns dedicated to catalytic determination on other probe configurations. 


\subsubsection{The Damköhler probes and the flat plate}

In order to understand better how non-equilibrium conditions can affect catalytic measurements in plasma wind tunnels, recombination coefficients are determined in different flow configurations. Such study was realized at stagnation point with the Damköhler probes, and at off-stagnation point with a linear calorimeter installed on a flat plate model.

The logic behind the experiments on the three Damköhler probes is to change the boundary layer thickness and modify diffusive characteristics in front of the calorimeter through $D a_{g}$. Thus, the Frozen probe leads to boundary layers with less gas-phase recombination and, conversely, the Equilibrium probe enhances recombination, with both probes taking Standard one as reference. Results show that, although the boundary layer thickness on the Standard probe is between the other two, the recombination coefficients $\gamma_{F R}$ and $\gamma_{E Q}$ are lower than $\gamma_{r e f}$ for most testing conditions. This is an unexpected result which requires a deeper analysis of the flow physics.

The background for such analysis was brought by Rosner [99], as explained in Sec. 4.1. He concluded that the Nusselt number based on the heat flux transferred to a catalytic cold wall is function of both $D a_{g}$ and $D a_{w}$. From a parametric analysis with NEBOULA, it is possible to conclude that the same behavior could be expected in Plasmatron tests. Then, the surprising results on both $\gamma_{F R}$ and $\gamma_{E Q}$ with respect to $\gamma_{r e f}$ can be explained by computing the non-dimensional numbers $N u, D a_{g}$ and $D a_{w}$ around the three Damköhler probes. The analysis shows that the recombination coefficients obtained on both Frozen and Equilibrium probes are consistent with Rosner's non-equilibrium analysis, meaning that experimentally determined recombination coefficients are the result of the whole non-equilibrium boundary layer configuration. Another important conclusion from these results is that, indeed, in order to apply an experimentally determined recombination coefficient to a CFD simulation, a proper flight extrapolation strategy for non-equilibrium boundary layers must be applied. The LHTS methodology introduced in this thesis applies to the stagnation point, and a new extrapolation strategy for shear flows has been developed in this dissertation.

In the framework of the IXV catalytic experiment CATE, a technique to measure catalycity at off-stagnation point is also of interest. This is assessed on the linear copper calorimeter available in VKI. The procedure is very similar to the one followed at stagnation point, where measured heat flux distributions along the calorimeter are compared to predictions from $\mathrm{CFD}++$. Results show that there is a transition from high to low catalytic conditions downstream of the stagnation point. This recombination coefficient evolution is consistent with the results previously discussed at stagnation point, where an increase in $D a_{g}$ leads to a reduction of $\gamma$ along the plate. One conclusion from this activity is that, indeed, catalysis can be determined at off-stagnation point in the Plasmatron. However, the work is much more tedious than for stagnation point, mainly due to the need of computing many flat plate CFD simulations under several catalytic conditions to generate a recombination coefficient map from which a $\gamma$ distribution along the plate can be extracted.

One final conclusion that should be mentioned from the Damköhler probes and the flat plate testing campaigns is that, although results are not consistent with current $\gamma$-models, they are compatible with the behavior expected from FRC models. It is important to mention that this fact does not mean that $\gamma$-models are obsolete, but that they have to be combined with proper flight 
extrapolation strategies to link the recombination coefficient on ground with the recombination coefficient in flight.

\subsubsection{The off-stagnation point extrapolation}

In harmony with the results and conclusions of experimental campaigns provided in the present dissertation and discussed above, a flight to ground extrapolation at off-stagnation point is required. This is developed in the context of the post-flight activities of CATE for aerothermodynamic model validation using plasma wind tunnels.

Following a similar analysis to that carried out at stagnation point with LHTS methodology, it turns out that the local duplication of the boundary layer downstream of the stagnation point of a flying body is possible at the trailing edge of a flat plate. This is achieved by using the boundary layer edge conditions at a specific point on the wall coordinate on the vehicle to define the testing conditions for a plasma wind tunnel. Variables involved in the method are provided in Table 5.2 .

The off-stagnation point extrapolation methodology is tested on IXV simulations along several wall coordinates: from $40 \mathrm{~cm}$ to $175 \mathrm{~cm}$ from the stagnation point. The same boundary layer is obtained at the end of a flat plate for most of the situations. It is shown that temperature, velocity and species profiles on the flat plate are in reasonable agreement with those on IXV. An exception is found for the velocity profile close to the stagnation point, where an effect of the entropy layer appearing after a bow shock is expected on the IXV simulations.

The high velocity reached downstream of the stagnation point in lifting body re-entry vehicles limits the application of the extrapolation methodology in the Plasmatron facility. However, this can be solved through the simplification of the extrapolation parameters, as proposed by Barbante [111, that allows scaling the flat plate model and adapt to a reachable velocity condition inside the facility. This scaling step assumes a flow with low Eckert numbers to relax the velocity duplication requirement, and focuses on the thermal and chemical aspects of the flow. For the specific case considered in the present work, it is shown that, indeed, the IXV temperature and species profiles can be obtained at the end of a $20 \mathrm{~cm}$ flat plate without significant differences in heat flux with respect the IXV.

A validation campaign of the off-stagnation point extrapolation methodology is carried out in the Plasmatron, in which the flow velocity is varied with the mass flow of gas injected into the test chamber. The same heat flux distribution along the convective time $x / U_{\infty}$ over the linear calorimeter is obtained for the two test configurations, showing outstanding Plasmatron capabilities for the post-flight activities required by CATE.

\subsubsection{The catalytic experiment CATE}

A detailed description of CATE implementation, together with trajectory data of IXV and temperature measurements of CATE are collected in $\mathrm{Ch}$. 6. The purpose is to make data available for the post-flight activities to be developed at VKI in the near future.

Information on TPS design, including thicknesses of the different insulating layers used, is provided for further thermal analysis of the system. As the temperatures are measured on the internal face of the shingle, this information is critical if one tries to define the actual surface temperatures 
on the side exposed to the plasma. For instance, a transfer function relating the measured temperature on CATE and the surface temperatures on the side in contact with the gas could be defined in the future.

Then, relevant flight conditions are also specified for flight extrapolation. Particularly, altitude and velocity data are used to define reference conditions for both stagnation and off-stagnation point extrapolating configurations. The peak heating point is taken as reference at stagnation point, while the maximum temperature jump caused by the low-to-high catalytic transition on CATE is taken at off-stagnation point.

Temperature measurements of CATE are provided at these conditions, showing the success of the flight experiment regarding catalytic phenomena evaluation. From these data, it is observed that thermal conduction inside the CMC panel could make temperature jumps smoother than the catalytic discontinuity imposed by the Mullite patch. This thermal conduction can also affect the temperature measurements available on the other side of IXV's symmetry plane.

It is observed also that the peak temperature does not appear just after the catalytic transition, but a bit further downstream. This fact contrasts with what had been anticipated by CFD simulations, where the peak temperature is obtained just after the catalytic transition. Such effect could be caused by a combination of atomic species diffusing towards the patch in the direction parallel to the wall, and the longer time that it takes for atomic species to recombine through a catalytic reaction compared to the convective time of the flow. However, a deeper analysis should be carried out in the future to validate these last statements, possibly with $3 \mathrm{D}$ computations on a catalytic transition in combination with a thermal conductivity model for the TPS.

\subsection{Future perspective}

The catalytic determination with the Mini-Max provides a range of possible recombination coefficients on copper. This range can be used to define an order of magnitude for the uncertainty around each $\gamma$ measurement, as seen in the testing campaign on the Damköhler probes. A dedicated uncertainty quantification campaign could have been very helpful to complement the results of the Mini-Max. The implementation of a polynomial chaos routine in Dakota software was attempted during the development of this dissertation and it turned unsuccessful, mainly due to instabilities of the CERBOULA code at intermediate values of catalysis, where outer edge enthalpy $H_{e}$ gradients with respect to $\gamma$ were at their highest values, and the $H_{e^{-}} \gamma$ relation had to be obtained with a constant interaction with the user during the application of the Mini-Max. Nevertheless, the uncertainty quantification is still ongoing at VKI and an improved implementation based on the Bayes rule is expected to provide additional information about the uncertainty around the Mini-Max method.

Next, a new FRC model for copper is planned for implementation in VKI non-equilibrium codes. This could bring valuable information to complement conclusions drawn from both the Damköhler probes and the flat plate measurements.

Finally, and with the goal of boundary layer duplication around CATE, a good reproduction of IXV's windward TPS should be obtained to test at off-stagnation point in the Plasmatron. This would require the design of a new probe that accommodates all the internal insulating layers specified 
in Table 6.2 if one tries to consider thermal conduction inside the system, which is neglected by most CFD solvers. Therefore, in the author's opinion, the first step for CATE's post-flight analysis should include a thorough characterization of the thermal conduction inside the TPS. 



\section{Appendix A.}

\section{Dynamic pressure measurements}

Table A.1.: Dynamic pressure measurements

\begin{tabular}{|c|c|c|c|c|c|c|c|c|}
\hline \multirow{3}{*}{ Test } & \multicolumn{2}{|c|}{15 mbar } & \multicolumn{2}{|c|}{$50 \mathrm{mbar}$} & \multicolumn{2}{|c|}{$100 \mathrm{mbar}$} & \multicolumn{2}{|c|}{$200 \mathrm{mbar}$} \\
\hline & $\dot{q}_{c w}^{(S T)}$ & $P_{d y n}$ & $\dot{q}_{c w}^{(S T)}$ & $P_{d y n}$ & $\dot{q}_{c w}^{(S T)}$ & $P_{d y n}$ & $\dot{q}_{c w}^{(S T)}$ & $P_{d y n}$ \\
\hline & $\mathrm{kW} \mathrm{m}^{-2}$ & $\mathrm{~Pa}$ & $\mathrm{~kW} \mathrm{~m}{ }^{-2}$ & $\mathrm{~Pa}$ & $\mathrm{~kW} \mathrm{~m}^{-2}$ & $\mathrm{~Pa}$ & $\mathrm{~kW} \mathrm{~m}{ }^{-2}$ & $\mathrm{~Pa}$ \\
\hline 1 & 303.35 & 118.07 & 315.37 & 25.19 & 314.19 & 8.25 & 309.98 & 4.07 \\
\hline 2 & 510.61 & 129.97 & 518.94 & 32.78 & 507.11 & 12.41 & 519.36 & 8.03 \\
\hline 3 & 695.97 & 173.94 & 720.46 & 53.72 & 713.89 & 14.56 & 732.15 & 12.90 \\
\hline 4 & 922.63 & 232.45 & 914.68 & 63.49 & 913.06 & 17.20 & 914.02 & 14.59 \\
\hline 5 & 1115.03 & 267.12 & 1102.65 & 70.31 & 1112.35 & 28.41 & 1131.57 & 15.94 \\
\hline 6 & 1320.10 & 292.32 & 1317.14 & 79.74 & 1317.86 & 38.82 & 1309.31 & 16.47 \\
\hline 7 & 1517.15 & 316.87 & 1518.08 & 98.21 & 1545.27 & 44.81 & 1499.57 & 18.77 \\
\hline 8 & 1719.45 & 353.76 & 1721.00 & 129.61 & 1729.66 & 50.31 & 1715.58 & 25.88 \\
\hline 9 & 2025.16 & 385.10 & 1999.23 & 173.68 & 2028.50 & 65.89 & 2005.93 & 36.35 \\
\hline
\end{tabular}

Table A.2.: Linear interpolation coefficients for dynamic pressure measurements $\left(\dot{q}_{c w}^{(i)}=y_{0}+m \dot{q}_{c w}^{(r e f)}\right)$

\begin{tabular}{lrrr}
\hline \hline$P_{s}$ & $y_{0}$ & $m$ & $\mathrm{R}^{2}$ \\
\hline 15 mbar & 65.857 & 0.1655 & 0.9827 \\
50 mbar & -10.389 & 0.0810 & 0.9339 \\
100 mbar & -7.320 & 0.0340 & 0.9672 \\
200 mbar & -1.230 & 0.0162 & 0.9102 \\
\hline \hline
\end{tabular}





\section{Appendix B.}

\section{NDP database for stagnation point test campaigns}

The following tables provide the NDP database that has been computed and used during the stagnation point campaigns, both for the Mini-Max and for the Damköhler probes. 
Table B.1.: NDP database generated with CoolFluid for Mini-Max methodology application

\begin{tabular}{|c|c|c|c|c|c|}
\hline Test & $\overline{\Pi_{1}}$ & $\Pi_{2}$ & $\Pi_{3}$ & $\Pi_{4}$ & $\Pi_{5}$ \\
\hline $1 \mathrm{a}$ & 0.4474 & 0.4120 & 0.6816 & 0.4755 & 0.4755 \\
\hline $2 a$ & 4471 & 0.3349 & 0.5437 & .3766 & .4354 \\
\hline $3 a$ & 0.4347 & 0.3137 & 0.5142 & 0.3407 & 0.4212 \\
\hline $4 a$ & 4285 & 0.3015 & 0.4924 & 0.3279 & 0.4267 \\
\hline $5 a$ & 4327 & 0.2813 & 0.4571 & 0.3207 & 0.4127 \\
\hline $6 a$ & 4771 & 0.2548 & 0.4129 & 0.3495 & 0.4248 \\
\hline $7 a$ & .4508 & 0.2828 & 0.4502 & 0.3525 & 0.4562 \\
\hline $8 a$ & 4317 & 0.3078 & 0.4813 & 0.3584 & 0.4472 \\
\hline $9 a$ & 3966 & 0.3567 & 0.5637 & 0.3628 & 0.4235 \\
\hline $1 b$ & 4432 & 0.3000 & 0.4954 & 0.3407 & 0.4488 \\
\hline $2 b$ & 4371 & 0.2825 & 0.4657 & 0.3112 & 0.4392 \\
\hline $3 b$ & 0.4211 & 0.2830 & 0.4694 & 0.2978 & 0.4267 \\
\hline $4 \mathrm{~b}$ & .4121 & 0.2849 & 0.4690 & 0.2965 & 0.4271 \\
\hline $5 b$ & 4137 & 0.2800 & 0.4578 & 0.3009 & 0.4267 \\
\hline $6 \mathrm{~b}$ & 4433 & 0.2632 & 0.4288 & 0.3245 & 0.4392 \\
\hline $7 \mathrm{~b}$ & 0.4386 & 0.2834 & 0.4638 & 0.3425 & 0.4784 \\
\hline $8 b$ & 4211 & 0.3129 & 0.5046 & 0.3532 & 0.4709 \\
\hline $9 \mathrm{~b}$ & 3971 & 0.3605 & 0.5814 & 0.3686 & 0.4566 \\
\hline $1 \mathrm{c}$ & 4359 & 0.2791 & 0.4647 & 0.3133 & 0.4478 \\
\hline $2 \mathrm{c}$ & 4258 & 0.2684 & 0.4460 & 0.2886 & 0.4386 \\
\hline $3 c$ & 0.4101 & 0.2739 & 0.4580 & 0.2794 & 0.4268 \\
\hline $4 c$ & 4006 & 0.2793 & 0.4682 & 0.2796 & 0.4230 \\
\hline $5 c$ & 3940 & 0.2857 & 0.4769 & 0.2833 & 0.4209 \\
\hline $6 c$ & 3926 & 0.2883 & 0.4789 & 0.2885 & 0.4263 \\
\hline $7 \mathrm{c}$ & 0.3942 & 0.2885 & 0.4754 & 0.2950 & 0.4233 \\
\hline $8 c$ & 0.4068 & 0.2826 & 0.4625 & 0.3083 & 0.4275 \\
\hline $9 \mathrm{c}$ & 0.4119 & 0.2947 & 0.4840 & 0.3294 & 0.4247 \\
\hline $1 d$ & 0.4134 & 0.2671 & 0.4460 & 0.2803 & 0.4342 \\
\hline $2 \mathrm{~d}$ & 0.4047 & 0.2697 & 0.4532 & 0.2731 & 0.4263 \\
\hline $3 \mathrm{~d}$ & 0.3934 & 0.2766 & 0.4679 & 0.2703 & 0.4263 \\
\hline $4 d$ & 0.3821 & 0.2847 & 0.4816 & 0.2697 & 0.4208 \\
\hline $5 \mathrm{~d}$ & 0.3747 & 0.2924 & 0.4949 & 0.2722 & 0.4182 \\
\hline $6 \mathrm{~d}$ & 0.3699 & 0.2989 & 0.5055 & 0.2758 & 0.4173 \\
\hline $7 \mathrm{~d}$ & 0.3662 & 0.3049 & 0.5141 & 0.2794 & 0.4164 \\
\hline $8 d$ & 3647 & 0.3098 & 0.5210 & 0.2844 & 0.4168 \\
\hline $9 \mathrm{~d}$ & 0.3638 & 0.3160 & 0.5274 & 0.2921 & 0.4216 \\
\hline
\end{tabular}


Table B.2.: Standard probe NDP database generated with CoolFluid during Damköhler probe testing

\begin{tabular}{lrrrrr}
\hline \hline Test & $\Pi_{1}$ & $\Pi_{2}$ & $\Pi_{3}$ & $\Pi_{4}$ & $\Pi_{5}$ \\
\hline 1a & 0.4509 & 0.5083 & 0.8493 & 0.5989 & 0.5571 \\
2a & 0.4480 & 0.3516 & 0.5713 & 0.4011 & 0.4469 \\
3a & 0.4427 & 0.3272 & 0.5334 & 0.3612 & 0.4308 \\
4a & 0.4332 & 0.3121 & 0.5115 & 0.3382 & 0.4198 \\
5a & 0.4285 & 0.3008 & 0.4911 & 0.3274 & 0.4305 \\
6a & 0.4321 & 0.2830 & 0.4600 & 0.3210 & 0.4138 \\
7a & 0.4739 & 0.2534 & 0.4089 & 0.3444 & 0.4302 \\
8a & 0.4592 & 0.2748 & 0.4422 & 0.3525 & 0.4602 \\
9a & 0.4303 & 0.3095 & 0.4836 & 0.3586 & 0.4465 \\
\hline 1b & 0.4435 & 0.3006 & 0.4962 & 0.3418 & 0.4490 \\
2b & 0.4369 & 0.2831 & 0.4666 & 0.3116 & 0.4392 \\
3b & 0.4256 & 0.2829 & 0.4694 & 0.3006 & 0.4295 \\
4b & 0.4164 & 0.2836 & 0.4693 & 0.2960 & 0.4241 \\
5b & 0.4117 & 0.2850 & 0.4695 & 0.2971 & 0.4319 \\
6b & 0.4127 & 0.2815 & 0.4606 & 0.3001 & 0.4279 \\
7b & 0.4267 & 0.2691 & 0.4383 & 0.3100 & 0.4282 \\
8b & 0.4470 & 0.2661 & 0.4350 & 0.3325 & 0.4347 \\
9b & 0.4317 & 0.2949 & 0.4801 & 0.3470 & 0.4760 \\
\hline 1c & 0.4345 & 0.2763 & 0.4601 & 0.3082 & 0.4454 \\
2c & 0.4241 & 0.2686 & 0.4465 & 0.2869 & 0.4368 \\
3c & 0.4086 & 0.2745 & 0.4595 & 0.2791 & 0.4261 \\
4c & 0.3995 & 0.2802 & 0.4696 & 0.2800 & 0.4227 \\
5c & 0.3933 & 0.2863 & 0.4776 & 0.2837 & 0.4206 \\
6c & 0.3918 & 0.2889 & 0.4791 & 0.2887 & 0.4253 \\
7c & 0.3951 & 0.2880 & 0.4740 & 0.2962 & 0.4232 \\
8c & 0.4089 & 0.2820 & 0.4614 & 0.3107 & 0.4288 \\
9c & 0.4110 & 0.2970 & 0.4878 & 0.3307 & 0.4244 \\
\hline \hline & & & & &
\end{tabular}


Table B.3.: Frozen probe NDP database generated with CoolFluid during Damköhler probe testing

\begin{tabular}{lrrrrr}
\hline \hline Test & $\Pi_{1}$ & $\Pi_{2}$ & $\Pi_{3}$ & $\Pi_{4}$ & $\Pi_{5}$ \\
\hline 1a & 0.2831 & 0.5794 & 1.3640 & 0.4687 & 0.4551 \\
2a & 0.2858 & 0.4497 & 1.0429 & 0.3487 & 0.4081 \\
3a & 0.2794 & 0.4162 & 0.9786 & 0.3143 & 0.3946 \\
4a & 0.2765 & 0.3971 & 0.9374 & 0.2979 & 0.3873 \\
5a & 0.2753 & 0.3849 & 0.9098 & 0.2911 & 0.3948 \\
6a & 0.2762 & 0.3692 & 0.8816 & 0.2878 & 0.3792 \\
7a & 0.2692 & 0.3600 & 0.7850 & 0.2889 & 0.3681 \\
8a & 0.3125 & 0.3455 & 0.7436 & 0.3429 & 0.4075 \\
9a & 0.3286 & 0.3674 & 0.8155 & 0.3692 & 0.4671 \\
\hline 1b & 0.2844 & 0.3987 & 0.9289 & 0.3103 & 0.4147 \\
2b & 0.2738 & 0.3777 & 0.8939 & 0.2778 & 0.3985 \\
3b & 0.2694 & 0.3701 & 0.8814 & 0.2690 & 0.3930 \\
4b & 0.2656 & 0.3724 & 0.8924 & 0.2680 & 0.3894 \\
5b & 0.2640 & 0.3716 & 0.8939 & 0.2689 & 0.3921 \\
6b & 0.2625 & 0.3651 & 0.8853 & 0.2693 & 0.3887 \\
7b & 0.2579 & 0.3625 & 0.8746 & 0.2698 & 0.3765 \\
8b & 0.2613 & 0.3642 & 0.8069 & 0.2895 & 0.3889 \\
9b & 0.3031 & 0.3595 & 0.7931 & 0.3462 & 0.4868 \\
\hline 1c & 0.2799 & 0.3634 & 0.8540 & 0.2791 & 0.4099 \\
2c & 0.2672 & 0.3529 & 0.8432 & 0.2545 & 0.3956 \\
3c & 0.2563 & 0.3803 & 0.9373 & 0.2629 & 0.3929 \\
4c & 0.2519 & 0.3861 & 0.9577 & 0.2641 & 0.3872 \\
5c & 0.2492 & 0.3917 & 0.9733 & 0.2678 & 0.3901 \\
6c & 0.2465 & 0.3968 & 0.9861 & 0.2717 & 0.3885 \\
7c & 0.2452 & 0.3948 & 0.9822 & 0.2739 & 0.3879 \\
8c & 0.2397 & 0.3933 & 0.9606 & 0.2725 & 0.3772 \\
9c & 0.2548 & 0.3826 & 0.8705 & 0.3014 & 0.3998 \\
\hline \hline & & & & &
\end{tabular}


Table B.4.: Equilibrium probe NDP database generated with CoolFluid during Damköhler probe testing

\begin{tabular}{lrrrrr}
\hline \hline Test & $\Pi_{1}$ & $\Pi_{2}$ & $\Pi_{3}$ & $\Pi_{4}$ & $\Pi_{5}$ \\
\hline 1a & 0.0204 & 0.0333 & 1.3580 & 0.0010 & 0.0165 \\
2a & 0.6283 & 0.2349 & 0.2451 & 0.3603 & 4.2630 \\
3a & 0.5485 & 0.2447 & 0.2675 & 0.3329 & 3.4838 \\
4a & 0.5149 & 0.2441 & 0.2771 & 0.3185 & 0.4236 \\
5a & 0.5915 & 0.2188 & 0.2738 & 0.3552 & 0.4380 \\
6a & 0.8048 & 0.1664 & 0.2275 & 0.4293 & 5.7045 \\
7a & 0.8441 & 0.1821 & 0.2323 & 0.4668 & 6.5364 \\
8a & 0.1632 & 0.2611 & 0.6363 & 0.0838 & 0.1040 \\
9a & 0.1159 & 0.3044 & 1.0708 & 0.0729 & 0.0816 \\
\hline 1b & 0.4764 & 0.2339 & 0.2806 & 0.2756 & 0.3836 \\
2b & 0.4154 & 0.2531 & 0.3299 & 0.2622 & 0.3689 \\
3b & 0.3641 & 0.2848 & 0.3886 & 0.2572 & 0.3545 \\
4b & 0.3415 & 0.3009 & 0.4179 & 0.2558 & 0.3625 \\
5b & 0.3527 & 0.2999 & 0.4123 & 0.2687 & 0.3631 \\
6b & 0.4553 & 0.2586 & 0.3775 & 0.3270 & 0.4245 \\
7b & 0.5381 & 0.2383 & 0.3329 & 0.3706 & 0.5351 \\
8b & 0.5091 & 0.2622 & 0.3367 & 0.3696 & 0.5147 \\
9b & 0.3617 & 0.3419 & 0.4133 & 0.3219 & 0.4204 \\
\hline 1c & 0.4196 & 0.2466 & 0.3234 & 0.2590 & 0.3727 \\
2c & 0.3290 & 0.2792 & 0.4077 & 0.2270 & 0.3398 \\
3c & 0.2941 & 0.3082 & 0.4742 & 0.2235 & 0.3322 \\
4c & 0.2755 & 0.3404 & 0.5381 & 0.2316 & 0.3320 \\
5c & 0.2624 & 0.3623 & 0.5795 & 0.2362 & 0.3359 \\
6c & 0.2705 & 0.3624 & 0.5612 & 0.2478 & 0.3403 \\
7c & 0.3123 & 0.3396 & 0.4981 & 0.2784 & 0.3638 \\
8c & 0.3577 & 0.3249 & 0.4555 & 0.3131 & 0.3853 \\
9c & 0.3098 & 0.3669 & 0.4900 & 0.2975 & 0.4189 \\
\hline \hline & & & & & \\
\hline
\end{tabular}





\section{Appendix C.}

\section{List of Damköhler probes' non-dimensional parameters}

Here, non-dimensional numbers computed with Mutation ++ using the results obtained during the Damköhler probes testing campaign are provided. Note that the reference gas reaction is: $2 \mathrm{~N}+\mathrm{N} \longrightarrow \mathrm{N}_{2}+\mathrm{N}$.

Table C.1.: Non-dimensional parameters obtained during Damköhler probes tests at 15 mbar

\begin{tabular}{lrrrrrrrrr}
\hline \hline Test & $D a_{w}^{(S T)}$ & $D a_{g}^{(S T)}$ & $N u^{(S T)}$ & $D a_{w}^{(F R)}$ & $D a_{g}^{(F R)}$ & $N u^{(F R)}$ & $D a_{w}^{(E Q)}$ & $D a_{g}^{(E Q)}$ & $N u^{(E Q)}$ \\
\hline 1a & 1.05686 & 0.1068 & 5.21 & 0.12525 & 0.0016 & 2.13 & - & - & - \\
2a & 0.60394 & 142.80 & 4.78 & 0.06783 & 5.2026 & 1.91 & - & - & - \\
3a & 0.50967 & 545.07 & 5.53 & 0.07766 & 21.535 & 2.22 & - & - & - \\
4a & 0.45011 & 968.86 & 6.18 & 0.08114 & 47.915 & 2.50 & - & - & - \\
5a & 0.40732 & 1360.74 & 6.76 & 0.09517 & 74.978 & 2.83 & - & - & - \\
$6 \mathrm{a}$ & 0.37497 & 1720.35 & 7.32 & 0.08306 & 120.57 & 2.95 & - & - & - \\
$7 \mathrm{a}$ & 0.36948 & 2284.60 & 8.36 & 0.08274 & 140.46 & 3.01 & - & - & - \\
8a & 0.30990 & 1984.39 & 8.11 & 0.09482 & 274.00 & 3.49 & - & - & - \\
$9 \mathrm{a}$ & 0.20419 & 877.14 & 6.43 & 0.07418 & 280.58 & 3.41 & - & - & - \\
\hline \hline
\end{tabular}

Table C.2.: Non-dimensional parameters obtained during Damköhler probes tests at 50 mbar

\begin{tabular}{lrrrrrrrrr}
\hline \hline Test & $D a_{w}^{(S T)}$ & $D a_{g}^{(S T)}$ & $N u^{(S T)}$ & $D a_{w}^{(F R)}$ & $D a_{g}^{(F R)}$ & $N u^{(F R)}$ & $D a_{w}^{(E Q)}$ & $D a_{g}^{(E Q)}$ & $N u^{(E Q)}$ \\
\hline 1b & 0.71695 & 250.72 & 3.08 & 0.09977 & 3.5714 & 1.56 & - & - & - \\
2b & 0.55455 & 5568.14 & 3.87 & 0.07000 & 163.42 & 1.82 & - & - & - \\
3b & 0.47957 & 13956.58 & 4.61 & 0.07166 & 552.00 & 2.15 & 0.07934 & 44638.19 & 3.89 \\
4b & 0.42952 & 22483.43 & 5.23 & 0.07189 & 1189.31 & 2.40 & 0.15876 & 67554.34 & 4.98 \\
5b & 0.39242 & 30792.55 & 5.78 & 0.09384 & 1683.59 & 2.75 & 0.25159 & 111290.17 & 6.22 \\
6b & 0.36161 & 38901.95 & 6.26 & 0.09991 & 2391.09 & 2.98 & 0.32400 & 271314.60 & 8.79 \\
7b & 0.33244 & 47380.77 & 6.66 & 0.08842 & 3188.80 & 3.02 & 0.32405 & 419404.96 & 11.11 \\
8b & 0.29746 & 49287.12 & 6.90 & 0.07628 & 4408.82 & 3.04 & 0.32181 & 358658.41 & 11.10 \\
9b & 0.20770 & 25480.04 & 5.78 & 0.06606 & 7038.40 & 3.29 & - & - & - \\
\hline \hline
\end{tabular}


Table C.3.: Non-dimensional parameters obtained during Damköhler probes tests at 100 mbar

\begin{tabular}{llrrrrrrrr}
\hline \hline Test & $D a_{w}^{(S T)}$ & $D a_{g}^{(S T)}$ & $N u^{(S T)}$ & $D a_{w}^{(F R)}$ & $D a_{g}^{(F R)}$ & $N u^{(F R)}$ & $D a_{w}^{(E Q)}$ & $D a_{g}^{(E Q)}$ & $N u^{(E Q)}$ \\
\hline 1c & 0.40781 & 5101.61 & 2.41 & 0.06730 & 143.08 & 1.38 & - & - & - \\
2c & 0.34409 & 41308.59 & 3.32 & 0.07224 & 1187.46 & 1.75 & 0.07771 & 158671.72 & 3.06 \\
3c & 0.29886 & 88897.59 & 3.97 & 0.07638 & 2886.46 & 2.04 & 0.12726 & 256946.93 & 3.74 \\
4c & 0.26697 & 141625.58 & 4.49 & 0.06863 & 6405.04 & 2.24 & 0.18165 & 333106.62 & 4.37 \\
5c & 0.24116 & 192218.85 & 4.91 & 0.05799 & 11879.93 & 2.38 & 0.19675 & 411510.24 & 4.84 \\
6c & 0.21841 & 236330.22 & 5.25 & 0.05681 & 16534.59 & 2.53 & 0.18194 & 590235.77 & 5.37 \\
7c & 0.19556 & 261838.90 & 5.47 & 0.04799 & 22266.60 & 2.57 & 0.21232 & 896746.72 & 6.46 \\
8c & 0.17208 & 254323.17 & 5.57 & 0.04769 & 24229.61 & 2.58 & 0.19076 & 1005714.13 & 7.07 \\
9c & 0.12019 & 127318.85 & 4.61 & 0.02120 & 21174.27 & 2.00 & - & - & - \\
\hline \hline
\end{tabular}




\section{Bibliography}

[1] John David Anderson. Hypersonic and high-temperature gas dynamics. AIAA Education Series. American Institute of Aeronautics and Astronautics, 2006. 2,22

[2] Roop N. Gupta, Jerrold M. Yos, Richard A. Thompson, and Kam-Pui Lee. A review of reaction rates and thermodynamic and transport properties for an 11-species air model for chemical and thermal nonequilibrium calculations to 30000k. Nasa-rp-1232, NASA Langley Research Center, Hampton, VA, United States, 1990. 5, 40, 52, 53, 62, 73

[3] Chul Park. Nonequilibrium hypersonic aerothermodynamics. NASA Ames Research Center, New York John Wiley \& Sons, 1989. 5, 40, 84

[4] Michael G. Dunn and Sang-Wook Kang. Theoretical and experimental studies of reentry plasmas. Nasa-cr-2232, State Univ. of New York; Buffalo, NY, United States, NASA, Washington, United States, April 1973. 5, 40

[5] J. D. Ramshaw. Self-consistent binary diffusion in multicomponent gas mixture. Journal of Non-Equilibrium Thermodynamics, 15(3):295-300, 1990. 5, 11, 30

[6] Alessandro Munafò. Multi-scale models and computational methods for aerothermodynamics. PhD thesis, von Karman Institute for Fluid Dynamics / École Centrale Paris, 2015. 5

[7] A. F. Kolesnikov. Conditions of simulation of stagnation point heat transfer from a highenthalpy flow. Fluid Dynamics (from Russian), 28(1):131-137, 1993. 6, 21, 79

[8] A. Kolesnikov. The concept of local simulation for stagnation point heat transfer in hypersonic flows: Application and validation. In 21 ${ }^{\text {st }}$ AIAA Aerodynamic Measurement Technology and Ground Testing Conference, Denver, CO, June 2000. AIAA Paper 2000-2515. 6, 21, 79

[9] A. Turchi, J. J. Matesanz Saiz, T. E. Magin, and O. Chazot. On the flight extrapolation of stagnation-point ablative material plasma wind tunnel tests. In $8^{\text {th }}$ European Symposium on Aerothermodynamics for Space Vehicles, Lisbon, Portugal, 2-6 March, 2015. ESA. 6, 21

[10] P. F. Barbante and O. Chazot. Flight extrapolation of plasma wind tunnel stagnation region flowfield. Journal of Thermophysics and Heat Transfer, 20(3):493-499, July 2006. 6, 19,21

[11] Francesco Panerai. Aerothermochemistry characterization of Thermal Protection Systems. $\mathrm{PhD}$ thesis, von Karman Institute for Fluid Dynamics / Università Degli Studi Di Perugia, 2012. 6, 7, 20, 27, 33, 45, 56, 79, 84, 88, 102

[12] A. Kolesnikov. Combined measurements and computations of high enthalpy plasma flows for determination of tpm surface catalycity. Rto en-8, rto avt course in Measurement Techniques 
for High Enthalpy and Plasma Flows held at the von karman institute, Intitute of Problems in Mechanics, 1999. 6

[13] Peter Atkins and Julio de Paula. Physical Chemistry. W. H. Freeman, $8^{\text {th }}$ edition, 2006. 10

[14] P. M. Chung. Chemically reacting non-equilibrium boundary layers. Advances in Heat Transfer, 1965. 10

[15] R. Goulard. On catalytic recombination rates in hypersonic stagnation heat transfer. Journal of Jet Propulsion, 28(11):737-745, 1958. 10, 21, 25, 33

[16] Lester Lees. Laminar heat transfer over blunt-nosed bodies at hypersonic flight speeds. Jet Propulsion, 26(4):259-269, 1956. 10

[17] J. A. Fay and F. R. Riddell. Theory of stagnation point heat transfer in dissociated air. Journal of the Aerospace Sciences, 25(2):73-85, 1958. 10, 21, 25

[18] James A. Fay and Nelson H. Kemp. Theory of stagnation point heat transfer in a partially ionized diatomic gas. AIAA Journal, 1(12):2741-2751, 1963. 10

[19] S. A. Vasil'evskii, A. F. Kolesnikov, and M. I. Yakushin. Determination of the effective probabilities of the heterogeneous recombination of atoms when heat flow is influenced by gas-phase reactions. High Temperature (from Russian), 29:411-419, 1991. 11

[20] Richard A. Copeland, Joan B. Pallix, and David A. Stewart. Surface-catalyzed production of no from recombination of $\mathrm{n}$ and o atoms. Journal of Thermophysics and Heat Transfer, 12(4):496-499, October-December 1998. 11, 17, 30

[21] C. D. Scott. Catalytic recombination of nitrogen and oxygen on high temperature surface insulation. In $13^{\text {th }}$ AIAA Thermophysics Conference, Snowmass, Colorado, 1980. AIAA 801477.

[22] P. F. Barbante. Accurate and efficient modelling of high temperature nonequilibrium air flows. PhD thesis, von Karman Institute for Fluid Dynamics / Université Libre de Bruxelles, 2001. 11, 28, 30

[23] R. M. Logan. Calculation of the energy accommodation coefficient using the soft-cube model. Surface Science, 15(3):387-402, 1969. 11

[24] J. R. Manson. Simple model for the energy accommodation coefficient. The Journal of Chemical Physics, 56(7):3451-3455, 1972.

[25] I. S. Altman, D. Lee, J. Song, and M. Choi. Experimental estimate of energy accommodation coefficient at high temperatures. Phys. Rev. E, 64:52202, October 2001. 11

[26] Young C. Kim and Michel Boudart. Recombination of o, n, and h atoms on silica: Kinetics and mechanisms. Langmuir, 7(12):2999-3005, 1991. 12 
[27] J. Thoemel, O. Chazot, and P. F. Barbante. Aspects of advanced catalysis modeling for hypersonic flows. Proceedings of the summer program, Center for Turbulence Research, 2008. 12

[28] Jan Thoemel. Surface catalysis in high enthalpy flows. PhD thesis, von Karman Institute for Fluid Dynamics / Rheinisch-Westfälische Technische Hochschule Aachen, 2012. 12

[29] J. M. Meyers, W. P. Owens, and D. G. Fletcher. Surface catalyzed reaction efficiencies in nitrogen and oxygen plasmas from laser induced fluorescence measurements. In $44^{\text {th }}$ AIAA Thermophysics Conference, San Diego CA, United States, June 24-27, 2013. AIAA 2013-3139. 12. 33

[30] W. A. Seward and E. J. Jumper. Model for oxygen recombination on silicon-dioxide surfaces. Journal of Thermophysics and Heat Transfer, 5(3):284-291, July-September 1991. 13

[31] J. C. Greaves and J. W. Linnett. The recombination of oxygen atoms at surfaces. Trans. Faraday Soc., 54:1323-1330, 1958. 13, 33

[32] F. Nasuti, M. Barbato, and C. Bruno. Material-dependent catalytic recombination modeling for hypersonic flows. Journal of Thermophysics and Heat Transfer, 10(1):131-136, JanuaryMarch 1996. 13

[33] M. Barbato, S. Reggiani, C. Bruno, and J. Muylaert. Model for heterogeneous catalysis on metal surfaces with applications to hypersonic flows. Journal of Thermophysics and Heat Transfer, 14(3):412-420, July-September 2000. 13

[34] I. Armenise, M. Barbato, M. Capitelli, and E. Kustova. State-to-state catalytic models, kinetics, and transport in hypersonic boundary layers. Journal of Thermophysics and Heat Transfer, 20(3):465-476, July 2006. 13

[35] S. Di Benedetto and C. Bruno. Novel semi-empirical model for finite rate catalysis with application to pm1000 material. Journal of Thermophysics and Heat Transfer, 24(1):50-59, January 2010. 13

[36] Jochen Marschall and Matthew MacLean. Finite-rate surface chemistry model, i: formulation and reaction system examples. In $42^{\text {nd }}$ AIAA Thermophysics Conference, Honolulu, Hawaii, 27-30 June 2011. AIAA 2011-3783. 14

[37] Matthew MacLean, Jochen Marschall, and David M. Driver. Finite-rate surface chemistry model, ii: coupling to viscous navier-stokes code. In $42^{\text {nd }}$ AIAA Thermophysics Conference, Honolulu, Hawaii, 27-30 June 2011. AIAA 2011-3784. 14

[38] Markus Fertig, Georg Herdrich, and Monika Auweter-Kurtz. $\mathrm{SiO}_{2}$ catalysis modeling for cfd calculations. In $39^{\text {th }}$ AIAA Thermophysics Conference, Miami, FL, 28-28 June 2007. AIAA. 14 
[39] M. Fertig. Finite rate surface catalysis modelling of pm1000 and sic employing the dlr cfd solver tau. In $8^{\text {th }}$ European Symposium on Aerothermodynamics for Space Vehicles, Lisbon, Portugal, 2-6 March, 2015. ESA. 14

[40] Cory Sorensen, Paolo Valentini, and Thomas E. Schwartzentruber. Uncertainty analysis of reaction rates in a finite-rate surface-catalysis model. Journal of Thermophysics and Heat Transfer, 26(3):407-416, July 2012. 14

[41] Bartomeu Massuti-Ballester and Georg Herdrich. Surface coverage and pressure dependent catalysis of copper. In $30^{\text {th }}$ International Symposium on Space Technology and Science, Kobe, Hyogo, Japan, 4-10 July, 2015. ISTS. 14,79

[42] A. Viladegut and O. Chazot. Enthalpy characterization and assessment of copper catalysis determination in inductively coupled plasma facility. In $45^{\text {th }}$ AIAA Thermophysics Conference, Dallas, Texas, 22-26 June, 2015. American Institite of Aeronautics and Astronautics. 14, 79

[43] M. Balat-Pichelin, M. Czerniak, and J.M. Badie. Thermal and chemical approaches for oxygen catalytic recombination evaluation on ceramic materials at high temperature. Applied Surface Science, 120(3-4):225-238, 1997. 15, 16, 50

[44] Marianne Balat-Pichelin and Florent Duqueroi. Heat transfer modeling at high temperature for the evaluation of atomic oxygen recombination energy on ceramic materials. International Journal of Thermal Sciences, 40:279-287, March 2001.

[45] M. Balat-Pichelin. Interaction of reactive gas flows and ceramics at high temperature: Experimental methods for the measurement of species recombination during planetary entry. In Experiment, Modeling and Simulation of Gas-Surface Interaction for Reactive Flows in Hypersonic Flights, pages 12 1-26. Educational Notes RTO-EN-AVT-142, Paper 12, 2007. 15

[46] M. Balat-Pichelin, J. M. Badie, R. Berjoan, and P. Boubert. Recombination coefficient of atomic oxygen on ceramic materials under earth re-entry conditions by optical emission spectroscopy. Chemical Physics, 291:181-194, June 2003. 16, 50

[47] Larbi Bedra and Marianne Balat-Pichelin. Comparative modeling study and experimental results of atomic oxygen recombination on silica-based surfaces at high temperature. Aerospace Science and Technology, 9:318-328, June 2005. 16

[48] M. Balat-Pichelin, L. Bedra, O. Gerasimova, and P. Boubert. Recombination of atomic oxygen on $\alpha-\mathrm{Al}_{2} \mathrm{O}_{3}$ at high temperature under air microwave-induced plasma. Chemical Physics, 340:217-226, November 2007. 16, 50,

[49] F. Nguyen-Xuan, O. Mallard, S. Cavadias, and J. Amouroux. Catalycity measurements on metallic and sic material surfaces in a pulsed plasma reactor. In $2^{\text {nd }}$ European Symposium on Aerothermodynamics for Space Vehicles, ESTEC, Noordwijk, The Nederlands, 21-25 November 1994. ESA SP-367. 16, 33 
[50] P. Cauquot, S. Cavadias, and J. Amouroux. Thermal energy accomodation from oxygen atoms recombination on metallic surfaces. Journal of Thermophysics and Heat Transfer, 12(2):206213, April-June 1998. 16, 33

[51] Joan B. Pallix and Richard A. Copeland. Measurement of catalytic recombination coefficients on quartz using laser-induced fluorescence. Journal of Thermophysics and Heat Transfer, 10(2):224-233, April-June 1996. 16

[52] David A. Stewart. Determination of surface catalytic efficiency for thermal protection materials - from room temperature to their upper use limit. In $31^{\text {st }}$ AIAA Thermophysics Conference, New Orleans, LA, June 1996. AIAA. 17, 18

[53] S. Sepka, Y.-K. Chen, J. Marschall, and R. A. Copeland. Experimental investigation of surface reactions in carbon monoxide and oxygen mixtures. Journal of Thermophysics and Heat Transfer, 14(1):45-52, January-March 2000. 17

[54] J. Marschall, R. A. Copeland, H. H. Hwang, and M. J. Wright. Surface catalysis experiments on metal surfaces in oxygen and carbon monoxide mixtures. In $44^{\text {th }}$ AIAA Aerospace Sciences Meeting and Exhibit, Reno, Nevada, 9-12 January 2006. AIAA 2006-181. 17

[55] J. Marschall. Laboratory determination of thermal protection system materials surface catalytic properties. In Experiment, Modeling and Simulation of Gas-Surface Interactions for Reactive Flows in Hypersonic Flights, pages 11 1-32. Educational Notes RTO-EN-AVT-142, Paper 11, 2007. 17

[56] Ernest L. Winkler and Roy N. Griffin Jr. Effects of surface recombination on heat transfer to bodies in a high enthalpy stream of partially dissociated nitrogen. Nasa-tn-d-1146, NASA Ames Research Center, Moffet Field, California, December 1961. 18

[57] Robert E. Sheldahl and Ernest L. Winkler. Effect of discontinuities in surface catalytic activity on laminar heat transfer in arc-heated nitrogen streams. Nasa-tn-d-3615, NASA Ames Research Center, Moffet Field, California, September 1966. 18

[58] David Stewart, Tahir Gökcen, Steven Sepka, Daniel Leiser, and Marc Rezin. Development of a catalytic coating for a shuttle flight experiment. In $10^{\text {th }}$ AIAA/ASME Joint Thermophysics and Heat Transfer Conference, Chicago, IL, 28 June-1 July 2010. AIAA 2010-4321. 18

[59] Eduardo Trifoni, Sara Di Benedetto, and Marco Di Clemente. A new global approach to evaluate the surface catalytic efficiency of tps materials based on arc jet experiments and numerical predictions. In $8^{\text {th }}$ European Symposium on Aerothermodynamics for Space Vehicles, Lisbon, Portugal, 2-6 March, 2015. ESA. 19

[60] O. Chazot, J. Thoemel, and M. Balat-Pichelin. Air catalycity determination in plasma wind tunnels and diffusion reactors. In $6^{\text {th }}$ European Symposium on Aerothermodynamics for Space Vehicles, Versailles, France, 3-6 November 2008. ESA SP-659, January 2009. 20 
[61] Işil Şakraker, Alessandro Turchi, and Olivier Chazot. Hypersonic aerothermochemistry duplication in ground plasma facilities: A flight-to-ground approach. Journal of Spacecraft and Rockets, 52(5):1273-1282, September 2015. 21

[62] Vladimir V. Lunev. Hypersonic Aerodynamics. Maschinostroenie, in Russian, 1975. 22

[63] Ernest V. Zoby. Empirical stagnation-point heat-transfer relation in several gas mixtures at high enthalpy levels. Nasa tn d-4799, Langley Research Center, Washington, D.C., 1968. 23. 25

[64] O. Chazot. Hypersonic stagnation point aerothermodynamics in plasmatron facilities. In Course on Hypersonic Entry and Cruise Vehicles, Standford University and NASA Ames Research Center, 2008. 23

[65] Chul Park, George A. Raiche, David M. Driver, Joseph Olejniczak, Imelda Terrazas-Salinas, T. Mark Hightower, and Takeharu Sakai. Comparison of enthalpy determination methods for arc-jet facility. Journal of Thermophysics and Heat Transfer, 20(4):672-679, October 2006. $25]$

[66] R. B. Pope. Measurements of enthalpy in low-density arc-heated flows. AIAA Journal, 6(1):103-110, 1968. 25

[67] Işil Şakraker. Aerothermodynamics of pre-flight and in-flight testing methodologies for atmospheric entry probes. PhD thesis, von Karman Institute for Fluid Dynamics / Université de Liège, 2016. 26, 27, 79

[68] P. Solano Lopez. Non-intrusive real time enthalpy determination in plasma flows. Stagiere report 2012-41, von Karman Institute for Fluid Dynamics, Rhode-Saint-Genèse, B1640, June 2012. 26

[69] D. G. Fletcher. Nonintrusive diagnostic strategies for arcjet stream characterisation. Rto en-8, rto avt course on Measurement Techniques for High Enthalpy and Plasma Flows held at the von karman institute, NASA Ames Research Center, 1999. 26

[70] Christophe O. Laux. Radiation and nonequilibrium collisional radiative models. Vki lecture series 2002-07, Physico-chemical models for high enthalpy and plasma flows held at the von karman institute, Rhode-Saint-Genèse, B1640, June 2002. 27

[71] James B. Scoggins and Thierry E. Magin. Development of mutation ++ : multicomponent thermodynamic and transport properties for ionized plasmas written in $\mathrm{c}++$. In $11^{\text {th }}$ AIAA/ASME Joint Thermophysics and Heat Transfer Conference, Atlanta, GA, 2014. American Institute of Aeronautics and Astronautics, AIAA 2014-2966. 27, 28, 93, 111

[72] Alessio Cipullo. Ground testing methodologies improvement in plasma wind tunnels using optical diagnostics. PhD thesis, Seconda Università degli Studi di Napoli / von Karman Institute for Fluid Dynamics, 2012. 27 
[73] Damien LeQuang. Spectroscopic measurements of sub-and supersonic plasma flows for the investigation of atmospheric re-entry shock layer radiation. PhD thesis, von Karman Institute for Fluid Dynamics / Université Blaise Pascal (UBP), 2014. 27, 28

[74] D. LeQuang, Y. Babou, and P. Andre. Experimental assessment of local thermodynamic equilibrium in the vki plasmatron air plasma jet. Journal of Technical Physics, 50(3):151162, 2009. 27

[75] S. Wüthrich. Numerical simulation of non-equilibrium chemically reacting hypersonic flows by means of a coupled Euler/boundary layer method. PhD thesis, EPFL, Lausanne, Switzerland, 1992. 29

[76] R. T. Davis. Numerical solution of the hypersonic viscous shock-layer equations. Journal of Spacecrafts and Rockets, 40(5):843-851, 1970. 29

[77] B. Bottin, D. P. Vanden Abeele, M. Carbonaro, G. Degrez, and G. S. R. Sarma. Thermodynamic and transport properties for inductively plasma modeling. Journal of Thermophysics and Heat Transfer, 13(3):343-350, 1999. 30

[78] Pietro Rini. Analysis of differential diffusion phenomena in high enthalpy flows, with application to Thermal Protection Material testing in ICP facilities. PhD thesis, von Karman Institute for Fluid Dynamics / Université Libre de Bruxelles, 2006. 31

[79] C. D. Scott. Wall catalytic recombination and boundary conditions in non-equilibrium hypersonic flows - with applications. In Advances in hypersonics. Vol. 2 - Modeling hypersonic flows, Boston, MA, United States, January 1992. Birkhaeuser. 31

[80] B. Bottin. Aerothermodynamic model of an Inductively-Coupled Plasma wind tunnel. PhD thesis, Université de Liège, von Karman Institute for Fluid Dynamics, 1999. 31, 40

[81] G. Kadavelil. Investigation on hypersonic stagnation heat transfer in plasmatron facility. Sr 2007-14, von Karman Institute for Fluid Dynamics, Rhode-Saint-Genèse, B1640, June 2007. 32

[82] J. C. Greaves and J. W. Linnett. Recombination of atoms at surfaces. part 5.-oxygen atoms at oxide surfaces. Trans. Faraday Soc., 55:1346-1354, 1959. 33

[83] Robert A. Young. Measurements of the diffusion coefficient of atomic nitrogen in molecular nitrogen and the catalytic efficiency of silver and copper oxide surfaces. The Journal of Chemical Physics, 34(4):1295-1301, 1961.

[84] P. G. Dickens and M. B. Sutcliffe. Recombination of oxygen atoms on oxide surfaces. part 1.-activation energies of recombination. Trans. Faraday Soc., 60:1272-1285, 1964.

[85] R. A. Hartunian, W. P. Thompson, and S. Safron. Measurements of catalytic efficiency of silver for oxygen atoms and the $\mathrm{O}_{-} \mathrm{O}_{2}$ diffusion coefficient. The Journal of Chemical Physics, 43(11):4003-4006, 1965. 
[86] Albert L. Myerson. Exposure-dependent surface recombination efficiencies of atomic oxygen. The Journal of Chemical Physics, 50(3):1228-1234, 1969.

[87] Gerald A. Melin and R. J. Madix. Energy accommodation during oxygen atom recombination on metal surfaces. Trans. Faraday Soc., 67:198-211, 1971.

[88] David M. Driver and Steve Sepka. Side arm reactor study of copper catalysis. In $45^{\text {th }} A I A A$ Thermophysics Conference, Dallas, Texas, 22-26 June, 2015. American Institite of Aeronautics and Astronautics. 33,50

[89] J.W. May and J.W. Linnett. Recombination of atoms at surfaces: An effusion method applied to oxygen atom recombination. Journal of Catalysis, 7(4):324-341, 1967. 33

[90] V. A. Bityurin, A. N. Bocharov, D. S. Baranov, A. V. Krasilnikov, V. B. Knotko, and Y. A. Plastinin. Experimental study of flow parameters and mhd generator models at high frequency plasmatron. In $15^{\text {th }}$ Internation Conference on MHD Energy Conversion and $6^{\text {th }}$ Workshop on Magnetoplasma Aerodynamics, Moscow, Russia, 2005.

[91] H. W. Krassilchikoff. Procedure for the determination of the cold copper catalycity. Project report 2006-18, von Karman Institute for Fluid Dynamics, Rhode-Saint-Genèse, B1640, June 2006. 35, 40, 56

[92] H. W. Krassilchikoff. Plasma tests development for thermal protection system design in real flight conditions. In Summaries of VKI's Doctoral Candidate Research. von Karman Institute for Fluid Dynamics, 2009-2010. 33

[93] V. L. Kovalev and A. F. Kolesnikov. Experimental and theoretical simulation of heterogeneous catalysis in aerothermochemistry (a review). Fluid Dynamics, 40:669-693, April 2005. 33

[94] François Sanson, Nadège Villedieu, Francesco Panerai, Olivier Chazot, Pietro M. Congedo, and Thierry E. Magin. Quantification of uncertainty on the catalytic property of reusable thermal protection materials from high enthalpy experiments. Experimental Thermal and Fluid Science, 82:414 - 423, 2017. 36, 63

[95] G. de Crombrugghe. Investigations of gas-surface interactions in plasma wind tunnels for catalycity characterization. Project report 2012-01, von Karman Institute for Fluid Dynamics, Rhode-Saint-Genèse, B1640, June 2012. 40, 41, 56

[96] R. V. Kovalev, V. I. Vlasov, and G. N. Zalogin. Experimental and numerical modelling of heterogeneous recombination for martian entry conditions. In $5^{\text {th }}$ European Conference for Aeronautics and Space Sciences, Munich, Germany, July, 2013. EUCASS 2013. 40

[97] B. Esser. Experimental determination of recombination coefficients at high pressures. In $8^{\text {th }}$ European Symposium on Aerothermodynamics for Space Vehicles, Lisbon, Portugal, 2-6 March, 2015. ESA. 45 
[98] A. Nawaz, D.M. Driver, I. Terrazas-Salinas, and S. Sepka. Surface catalysis and oxidation on stagnation point heat flux measurements in high enthalpy arc jets. In $44^{\text {th }}$ AIAA Thermophysics Conference, San Diego CA, United States, June 24-27, 2013. AIAA 2013-3138. 50

[99] Daniel E. Rosner. Scale effects and correlations in nonequilibrium convective heat transfer. AIAA Journal, 1(7):1550-1555, July 1963. 50, 51, 65, 66, 77, 86, 124

[100] J. E. Broadwell. A simple model of the non-equilibrium dissociation of a gas in couette and boundary layer flows. Journal of Fluid Mechanics, 4(2):113-139, 1958. 50

[101] M. Capitelli, C. Gorse, S. Longo, and D. Giordano. Collision integrals of high-temperature air species. Journal of Thermophysics and Heat Transfer, 14(2):259-268, April-June 2000. 53

[102] J. R. Busing. The effect of catalytic surfaces on stagnation point heat transfer in partially dissociated flows. In The high temperature aspects of hypersonic flows - Proceedings of the AGARD-NATO specialists' meeting, pages 681-691, Rhode-Saint-Genèse, Belgium, 3-6 April, 1962. Pergamon Press. 54

[103] S. Herpin. Chemical aspects of hypersonic stagnation point heat transfer. Project report 2005-10, von Karman Institute for Fluid Dynamics, Rhode-Saint-Genèse, B1640, June 2005. 55

[104] A. Vanaerschot. Development of a flat plate probe for off-stagnation testig of space reentry vehicles. Master thesis project, von Karman Institute for Fluid Dynamics / Faculteit Ingenieurswetenschappen - Katholieke Universiteit Leuven, 2009-2010. 56, 57, 58, 59

[105] A. Braun. Validation of a methodology for off-stagnation point testing. Study thesis, von Karman Institute for Fluid Dynamics / Institute for Space Systems - Stuttgart University, 2011. 58,95

[106] Alan Viladegut and Olivier Chazot. Off-stagnation point testing in plasma facility. In Array, editor, EUCASS Proceedings Series - Advances in AeroSpace Sciences, volume 7, pages 113122, 2015. 58, 88

[107] N. Joiner, B. Esser, M. Fertig, A. Gülhan, G. Herdrich, and B. Massuti-Ballester. Development of an innovative validation strategy of gas-surface interaction modelling for re-entry applications. CEAS Space Journal, 8(4):237-255, 2016. 77, 79

[108] Alan Viladegut, Ümran Düzel, and Olivier Chazot. Diffusion effects on the determination of surface catalysis in inductively coupled plasma facility. Chemical Physics, 485âĂŞ486:88 - 97, 2017. 88

[109] Ümran Düzel. Validation of gas surface interaction modeling in plasmatron facility for in-flight experiment development. Project report 2015-14, von Karman Insititute for Fluid Dynamics, Rhode-Saint-Genèse, B1640, June 2015. 79, 88 
[110] Bernd Helber. Material response characterization of low density ablators in atmospheric entry plasmas. PhD thesis, von Karman Institute for Fluid Dynamics / Vrije Universiteit Brussels (VUB), 2016. 79

[111] P. F. Barbante. Heat flux duplication between ground facility and hypersonic flight. Journal of Thermophysics and Heat Transfer, 23(4):684-692, October 2009. 79, 81, 82, 84, 125

[112] Giuseppe Ceglia, Eduardo Trifoni, Jean-Baptiste Gouriet, Olivier Chazot, Vincenzo Mareschi, Giuseppe Rufolo, and Giorgio Tumino. Experimental investigation on ixv tps interface effects in plasmatron. CEAS Space Journal, 8(2):65-75, 2016. 88

[113] A. Cipullo. Plasma flow characterization by means of optical emission diagnostics. Project report 2010-05, von Karman Institute for Fluid Dynamics, Rhode-Saint-Genèse, B1640, June 2012. 93, 94

[114] F. Baldani. Vki plasmatron jet investigation for off-stagnation point testing. Project report 2009-21, von Karman Institute for Fluid Dynamics, Rhode-Saint-Genèse, B1640, June 2009. 93,94

[115] A. Viladegut. Off-stagnation point testing in plasma wind tunnel for ground-to-flight extrapolation. Project report 2012-06, von Karman Institute for Fluid Dynamics, Rhode-SaintGenèse, B1640, June 2012. 97

[116] Alan Viladegut, F. Panerai, O. Chazot, T. Pichon, P. Bertrand, C. Verdy, and C. Coddet. Design, integration and preliminary results of the ixv catalysis experiment. CEAS Space Journal, 9:141-151, June 2017. 102

[117] D. Stewart, J. Rakich, and M. Lanfranco. Catalytic surface effects experiment on the space shuttle. In $16^{\text {rd }}$ Thermophysics Conference, Palo Alto, California, 1981. AIAA 81-1143. 102

[118] D. Stewart and P. Kolodziej. Wall catalysis experiment on afe. In 23 ${ }^{\text {rd }}$ Thermophysics, Plasmadynamics and Lasers Conference, San Antonio, TX, 1988. AIAA 88-2674. 102

[119] Darryl P. Butt, John J. Mecholsky, Mark van Roode, and Jeffrey R. Price. Effects of plasmasprayed ceramic coatings on the strength distribution of silicon carbide materials. Journal of the American Ceramic Society, 73(9):2690-2696, 1990. 103

[120] A. K. Samanta, K. K. Dhargupta, and S. Ghatak. In-situ development of sic-mullite composites in ambient atmosphere from sic and al-hydroxyhydrogel powder precursor. Journal of Materials Science Letters, 20:2077-2080, 2001. 103

[121] Francesco Panerai and Olivier Chazot. Characterization of gas/surface interactions for ceramic matrix composites in high enthalpy, low pressure air flow. Materials Chemistry and Physics, 134(2-3):597 - 607, 2012. 103

[122] Kang N. Lee, Robert A. Miller, and Nathan S. Jacobson. New generation of plasma-sprayed mullite coatings on silicon carbide. Journal of the American Ceramic Society, 78(3):705-710, 1995. 106 
[123] C. V. Cojocaru, Y. Wang, C. Moreau, R. S. Lima, J. Mesquita-Guimarães, E. Garcia, P. Miranzo, and M. I. Osendi. Mechanical behavior of air plasma-sprayed ysz functionally graded mullite coatings investigated via instrumented indentation. Journal of Thermal Spray Technology, 20(1):100-107, 2011. 106

[124] Kang N. Lee and Robert A. Miller. Oxidation behavior of muilite-coated sic and sic/sic composites under thermal cycling between room temperature and $1200^{\circ}-1400^{\circ} \mathrm{c}$. Journal of the American Ceramic Society, 79(3):620-626, 1996. 106

[125] Kang N. Lee, Dennis S. Fox, Jeffrey I. Eldridge, Dongming Zhu, Raymond C. Robinson, Narottam P. Bansal, and Robert A. Miller. Upper temperature limit of environmental barrier coatings based on mullite and bsas. Journal of the American Ceramic Society, 86(8):12991306, 2003. 106

[126] Aaron M. Brandis and Christopher O. Johnston. Characterization of stagnation-point heat flux for earth entry. In $45^{\text {th }}$ AIAA Plasmadynamics and Lasers Conference, Atlanta, GA, 2014. American Institute of Aeronautics and Astronautics, AIAA 2014-2374. 111

[127] Fabio Pinna. Numerical analysis of gas-surface interaction for the thermal protection system of the ixv vehicle. Technical report, von Karman Institute for Fluid Dynamics, Rhode-SaintGenèse, B1640, Octiber 27, 2012. 117

[128] B. Harnisch, B. Kunkel, M. Deyerler, S. Bauereisen, and U. Papenburg. Ultra-light weight c/sic mirrors and structures. Esa bulletin 95, ESA - ESTEC, Noordwijk, The Netherlands, 1998. 119

[129] Javier de Cáceres. Cate post-flight data analysis. Project report 2016-06, von Karman Insititute for Fluid Dynamics, Rhode-Saint-Genèse, B1640, June 2016. 120 
